\title{
MASTER
}

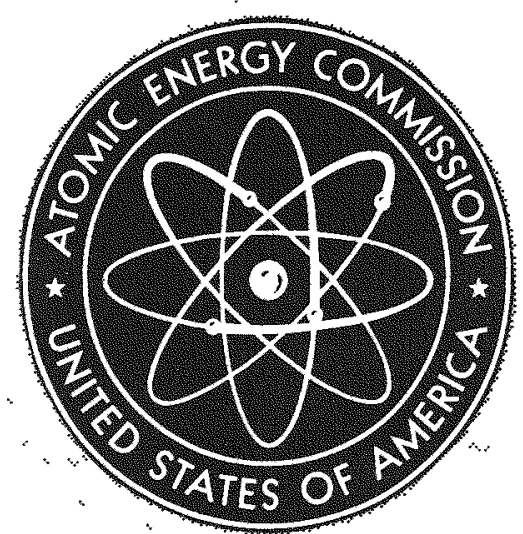

MND-P-3009-1

PHYSICS AND MATHEMATICS

\section{SNAP PROGRAMS}

Quarterly Progress Report No. 1, Tasks 3, 5 and 6 [for] October 22-December 31, 1959

November 1960

[OTI Issuance Date]

Nuclear Division

Martin Company

Baltimore, Maryland 


\section{DISCLAIMER}

This report was prepared as an account of work sponsored by an agency of the United States Government. Neither the United States Government nor any agency Thereof, nor any of their employees, makes any warranty, express or implied, or assumes any legal liability or responsibility for the accuracy, completeness, or usefulness of any information, apparatus, product, or process disclosed, or represents that its use would not infringe privately owned rights. Reference herein to any specific commercial product, process, or service by trade name, trademark, manufacturer, or otherwise does not necessarily constitute or imply its endorsement, recommendation, or favoring by the United States Government or any agency thereof. The views and opinions of authors expressed herein do not necessarily state or reflect those of the United States Government or any agency thereof. 


\section{DISCLAIMER}

Portions of this document may be illegible in electronic image products. Images are produced from the best available original document. 


\section{LEGAL NOTICE}

This report was prepared as an account of Government sponsored work. Neither the United States, nor the Commission, nor any person acting on behalf of the Commission:

A. Makes any warranty or representation, expressed or implied, with respect to the accuracy, completeness, or usefuness of the information contained in this report, or that the use of any information, apparatus, method, or process disclosed in this report may not infringe privately owned rights; or

B. Assumes any labilties with respect to the use of, or for damages resulting from the use of any information, apparatus, method, or process disclosed in inis report.

As used in the above, "person acting on behalf of the Commission" includes any employee or contractor of the Commission, or employee of such contractor, to the extent that such employee or contractor of the Commission, or employee of such contractor prepares, disseminates, or provides access to, any information pursuant to his employment or contract with the Commission, or his employment with such contractor.

This report has been reproduced directly from the best available copy.

Printed in USA. Price $\$ 3.50$. Available from the Office of Technical Services, Department of Commerce, Washington 25, D. C. 
MND-P-3009-1

\section{SNAP PROGRAMS}

\section{QUARTERLY PROGRESS REPORT NO. 1}

TASKS 3, 3 and 6

OCTOBER 22 - DECEMBER 31,1959

This work done under

Coniract AT (30-3)-217

for the AEC

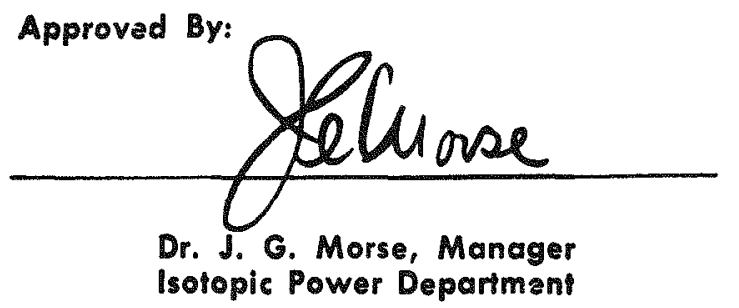

Nuclear Division

Martin Company

Baltimore, Maryland 
Blank page 


\section{FOREWORD}

This is the first quarterly progress report submitted to the Atomic Energy Commission under Tasks 3,5 and 6 of Contract AT (30-3)-217. It covers the work accomplished by The Martin Company Nuclear Division on these tasks during the period from October 22 to December 31 , 1959. 
Blank page 


\section{CONTENTS}

Page

Foreword .................. v v

Contents $\ldots \ldots \ldots \ldots \ldots \ldots \ldots \ldots \ldots$ vii

Summary ...................... $x i$

Introduction $\ldots \ldots \ldots \ldots \ldots \ldots \ldots \ldots \ldots \ldots \ldots$

I. Task $3--$ SNAP III Generator $\ldots \ldots \ldots \ldots \ldots \ldots \ldots$

A. Analysis of Generator Failure ........... 1

B. Test Revision .................. 4

II. Subtask 5.1--Advanced Thermoelectric Power System . 7

A. Status of the Westinghouse Generator ....... 7

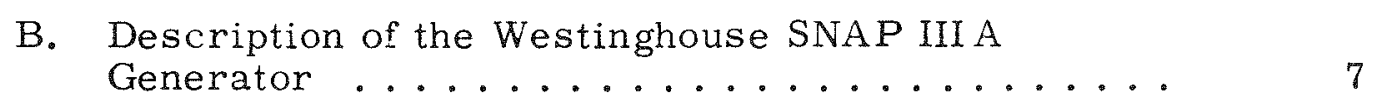

C. Performance Evaluation Program......... 10

III. Subtask 5.2--Basic Thermionic Development ...... 13

A. Cesium Diodes ................. 13

B. Effect of Cesium on Materials ........... 21

C. Electrical Heaters ............... 24

D. Diffusion of Gases Through Metals ........ 29

E. Work Function Tests .............. 30 


\section{CONTENTS (continued)}

Page

IV. Subtask 5.3- - Development of Low Power Thermionic

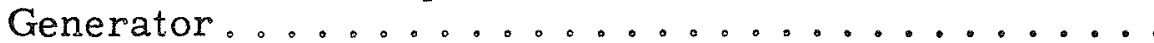

A. Thermoelectron Engineering Corporation Efforts .

B. Martin Nuclear Division Efforts ...........

V. Subtask 5.5--Operational Thermoelectric 2- to 5-Watt

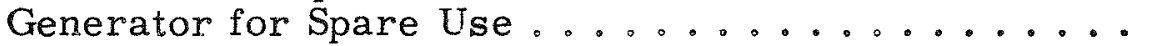

A. Generator Operational Tests ............

B. System Conceptual Design .............

VI. Subtask 5.6--1.0-Watt Nuclear Power Supply, Space

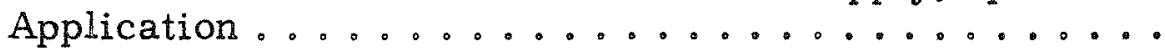

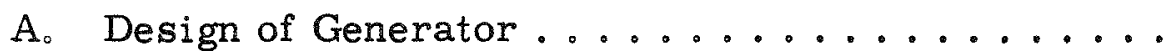

B. Thermal Analysis .................

VII. Subtask 5.7--100-Watt Thermoelectric Generator Con-

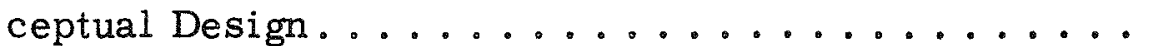

A. Thermoelectric and Heat Transfer Analysis .....

B. Generator and Component Design ..........

C. Component Tests and Measurements ........

D. Radiation Shielding Design.............

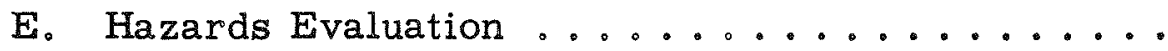

VIII. Subtask 5.8--Conceptual Design of 13-Watt Thermo-

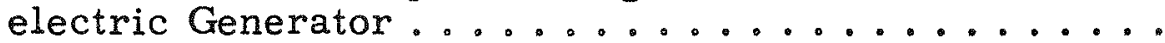

A. Heat Transfer and Thermoelectric Analysis .....

B. Generator and Component Design Studies ...... 


\section{CONTENTS (continued)}

Page

C. Nuclear Radiation Shielding .............. 185

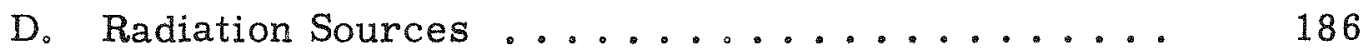

E. Hazards Evaluation .................. 190

IX. Subtask 6.1--General Development and Materials Re-

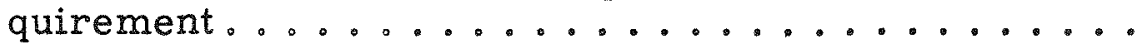

A. Materials Evaluation ................ 193

B. Process Selection ................... 198

X. Subtask 6.2--Americium and Curium Radioisotope Preparation Processes ................

A. Americium Purification and Conversion........ 201

B. Americium Slug Fabrication ............ 201

C. Purification of Curium .............. 209

D. Aluminum Volatilization Process .......... 210

XI. Subtask 6.3--Fuel Form ................ 221

XII. Subtask 6.4--Hazards Analysis and Shielding ...... 223

XIII. Subtask 6.5--Radiation Damage Testing ......... 227

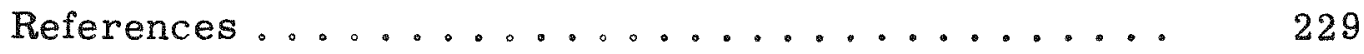

Appendix A ......................... A

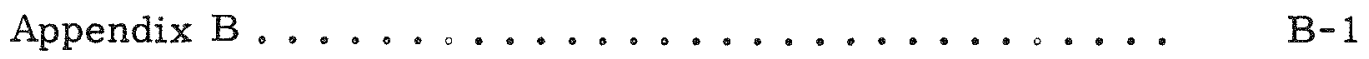

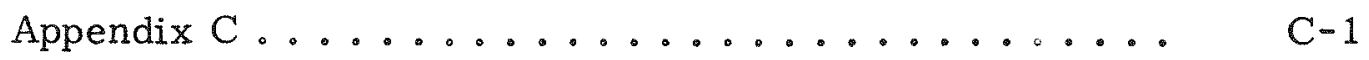

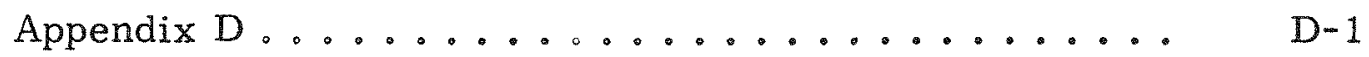

Appendix E ............................ E-1

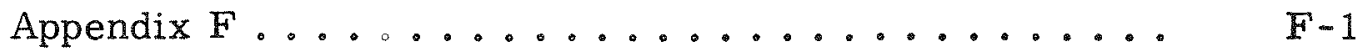


Blank page 


\section{SUMMARY}

\section{TASK 3--SNAP III GENERATOR}

Parametric testing was not resumed during this period since the test generator, which failed late in August, had not been repaired. However, an evaluation of the failed unit indicated that repair was feasible and parts were ordered for this purpose. The failure appeared to have been caused by operating the unit for considerable periods of time at excessive hot junction temperatures, as indicated by considerable sublimation of the thermal elements.

A new test specification was written for evaluation of the performance of small thermoelectric generators. This specification defines the procedures in detail and is designed to prevent overheating of the hot junctions.

The order has been placed for the new thermoelectric generator manufactured by Minnesota Mining and Manufacturing Company for use in the generator life test.

\section{SUBTASK 5.1--ADVANCED THERMOELECTRIC POWER SYSTEM}

The Westinghouse SNAP III-A thermoelectric generator was assembled and tested. A leak developed in the power flattening mechanism. Attempts were made to repair the leak, and the generator was delivered to Martin Nuclear Division. When it was discovered that the leak persisted, the generator was returned to Westinghouse for complete repair, along with a request for an operating manual and a complete set of drawings. This was the status at the close of the reporting period.

\section{SUBTASK 5.2--BASIC THERMIONIC DEVELOPMENT}

Work was performed under this subtask in the following areas:
A. Cesium Diodes
B. Effects of Cesium on Materials
C. Electrical Heaters
D. Diffusion of Gasses Through Metals
E. Work Function Study. 


\section{A. CESIUM DIODES}

Work was performed on four different types of cesium diodes during this reporting period. These are:

(1) Close-spaced cesium diode.

(2) Wire emitter cesium diode.

(3) Ribbon-emitter cesium diodes.

(4) Electron-heated cesium diodes.

The close-spaced cesium diode tests revealed that the cesium pressure in the vicinity of the interelectrode spacing was much less than the pres sure indicated by the bulb temperature, due to the fact that the interelectrode spacing is many times less than the mean free path of the cesium gas molecules. This indicates that results cannot be interpreted from the experimental data. The tests with the wire-emitter cesium diode were concerned with high temperature work. Attempts to conduct tests using these diodes were not too successful because of high temperature structural problems encountered in the tubes.

Work on the ribbon-emitter cesium diode was aimed principally at determining the feasibility of a ribbon-emitter tube. The tests conducted indicate that such diodes are feasible.

Work on the electron-heated cesium diode was in investigating and testing to determine the best approach to the problem of building such diodes.

\section{B. EFFECTS OF CESIUM ON MATERIALS}

Two tubes were constructed that contained various samples of materials being considered for use in cesium thermionic diodes. The materials in the tubes were exposed to a cesium atmosphere at elevated bulb temperatures. The tubes were cut open and the samples sent out for analysis. One observation readily apparent was that there was a rapid reaction between the glass bulbs and the cesium. Therefore, in subsequent tests, metal casings probably made of copper will be substituted for the glass bulbs.

\section{ELECTRICAL HEATERS}

Two types of electrical heaters were investigated during the quarter. The first type has the heating wire fitted into helical grooves cut in a 
ceramic cylinder. The second design consists of a metallized ceramic cylinder with helical grooves cut in the metallized surfaces. Tests made to date indicate that the metallized heater is the most promising from the standpoint of ruggedness and reliability.

\section{DIFFUSION OF GASES THROUGH METALS}

This work is being carried on to serve as a basis for obtaining better vacuums and being able to sustain these vacuums for longer periods of time. The work during this period was principally concerned with conducting a literature search and compiling information.

\section{E. WORK FUNCTION TESTS}

Considerable effort was expended in this area in an attempt to obtain a collector work function less than 2.0 volts. Several types of collectors were tested. In particular, very significant results were obtained for a molybdenum collector used in conjunction with an impregnated emitter.

A stable work function of 1.82-1.85 volts was achieved for an extended period of time. The key to this success lies in the outgassing procedure and the careful temperature control of the electrodes during activation.

\section{SUBTASK 5.3--DEVELOPMENT OF LOW POWER THERMIONIC GENERATOR}

Plans to fuel a thermionic unit with a Cerium-144 heat source were cancelled since it was felt that much more could be gained by continuing the work with electrically heated units.

\section{A. TEE CO'S EFFORT}

The major effort under this subtask was devoted to prototype development. Unit $1 \mathrm{~A}$ was well along toward completion when leaks developed in the leadthroughs. Further tests on the type of leadthrough used in the prototype revealed that they were not adequate. New types of leadthroughs were evaluated.

Heat transfer study and tests were conducted which were concerned primarily with the temperature drop across the film boundaries in the vicinity of the emitter. Two approaches were found to be effective. The first approach consists of using molybdenum dust between the surfaces; the second, of applying increased pressure across the film. 
Vacuum tests in various prototype shells were conducted to determine the degree of vacuum that can be maintained in a prototype shell containing typical leadthroughs, crimpoff tubes, etc. The effects of the gettering action due to the titanium shells being raised to elevated temperature for short periods of time were also studied. The titanium shell was found to be a vigorous getter and improved the vacuum considerably.

\section{B. MARTIN NUCLEAR EFFORT}

A molybdenum fuel capsule suitable for fueling with $\mathrm{Ce}-144$ fuel pellets to serve as the heat source for a thermionic generator was successfully developed. Burst tests were conducted to determine the structural integrity of the weld.

A welding fixture to remotely heliarc weld molybdenum fuel capsules was designed, developed, fabricated and tested during this period. The technology required to successfully heliarc weld the fuel capsules was also developed. Tests were run to determine the structural strength of such molybdenum welds. However, the results were too sporadic to draw definite conclusions. Burst tests on the capsule itself revealed that the welded capsule had sufficient strength. Considerable progress was accomplished in controlling grain size in the welded area.

A hazards report was written during this reporting period describing the hazards associated with a Cerium-144-fueled thermionic generator. A containment cask for the fueled unit was designed and fabricated. The fabrication of the Cerium-144 fuel pellets and the procedures to be followed in fueling a thermionic unit were coordinated with ORNL.

\section{SUBTASK 5.5--OPERATIONAL THERMOELECTRIC 2- to 5-WATT GENERATOR FOR SPACE USE}

The objectives of this subtask are to prove the operational capabilities of the SNAP III-type generator through tests simulating the anticipated environments and to develop conceptual system designs for this type generator. The generator test program is subdivided by the environments existing during the mission phases.

\section{A. OPERATIONAL ENVIRONMENTS}

The tests of the electrically heated generators to the vehicle-induced accelerations, vibrations and shocks were completed with satisfactory performance throughout. These tests are described in MND-P-2101, a three-volume report. 
The first two phases of the altitude chamber tests on a unit with a bare external surface and a unit with a painted surface have been completed with satisfactory results.

The controlled gas leak method of power flattening was proven to be feasible for periods of up to $70 \%$ of the radiosotope half life. A life test of the generator and orifice combination was initiated.

The effectiveness of various flame-sprayed coatings on the sublimation rate of lead telluride thermoelectric elements was evaluated to select superior materials and processes for further testing in an electrically heated fixture.

\section{B. MISSILE FAILURE ENVIRONMENTS}

The statements of work and the final cost estimate for the tests simulating launch pad vehicle failure were submitted to the New York Operations Office of the AEC for approval.

The impact tests were completed at Aberdeen Proving Ground. The simulated generator specimens survived with only minor deformation of the isotope capsule. The bare isotope capsule specimens failed to impact on the target due to unsatisfactory design of the track hardware. These tests will be repeated at a future date with a redesigned sled and specimen holder.

Two phases of the corrosion tests are complete. Oxidizing atmosphere results indicate that the Haynes 25 specimens developed an oxide coat that appeared to inhibit further corrosion while the stainless steel specimens were highly sensitized. Salt water tests are complete and the specimens are being prepared for metallographic examination. The salt spray tests are still in process.

Preliminary ejection test results are within $4 \%$ of the initial velocity required for a horizontal range of 200 feet. The thruster gun is currently being modified to effect a $15 \%$ increase.

\section{RE-ENTRY ENVIRONMENTS}

An analysis of the aerodynamic burnup characteristics of the generator has been completed. A subcontract to conduct plasma jet tests has been negotiated with the General Electric Aeroscience Laboratory and submitted to the New York Operations Office for approval.

The internal pressure test facility fabrication is complete except for some components scheduled for delivery early next period. 
A preliminary design of a shaped charge was completed and tested with satisfactory penetration of a steel plate.

\section{CONCEPTUAL DESIGN}

The intent of the second phase of this program is to complete conceptual designs using the SNAP III-type generator as a means of providing a short lead time system for any low powered space application.

1. Cerium-Fueled System

The information for reports on this system is assembled, but evaluation must await completion of the test phase.

2. Polonium-Fueled System

A simplified analysis of the temperature environment of a generator mounted in a missile nose cone during ascent appears to offer a solution. The stress produced in the isotope capsule material by the formation of helium was calculated and compared to the boiler code allowable stresses for pressure vessels. Various mounting arrangements are being studied.

\section{Converter and Battery System}

A study was initiated with a literature search of battery performance and converter design information.

\section{SUBTASK 5.6--1.0 WATT NUCLEAR POWER SUPPLY SPACE APPLICATION}

The purpose of Subtask 5.6 is to analyze and design a 1-watt (e) power supply system consisting of a Plutonium-238-powered generator and a static voltage converter. The electrical conversion components are series-connected lead telluride thermoelectric semiconductors. The technologies developed for the SNAP III and Strontium-90 generators under Atomic Energy Commission contracts have been utilized in the design of the proposed unit.

The generator developed under this task will be optimized in power source, electric conversion system, and radiator design, and will have a safe unattended life of from 2 to 5 years in a space environment. Based on the heat transfer analysis of the preliminary generator configuration, $4.9 \%$ of the nuclear source heat is converted to electrical energy.

The work planned and accomplished during this quarter covers the design and analysis of a preliminary generator design. 


\section{SUBTASK 5.7--100-WATT THERMOELECTRIC GENERATOR CONCEPTUAL DESIGN}

The objective of this program is to develop a conceptual design of a thermoelectric generator fueled with Curium-242 which will produce 100 watts of electrical power for a space application. Work performed during the period October to December 1959 was largely devoted to heat transfer analysis, isotope requirements, basic configuration design studies, selection and optimization of thermoelectric generator parameters, and radiation shield design. Evaluation of fuel containment problems under conditions of helium evolution and a program to measure thermoelectric materials data were also undertaken.

Several general types of generator designs were compared in order to select the one most advantageous from the standpoints of reliability, weight, efficiency, and ease of fabrication. The designs were based on the following systems:

(1) Liquid convection to remove heat from the hot junctions of the thermoelements to the radiating surface.

(2) Radiative transfer of heat from the hot junction, with biological shielding (required before launch) contained entirely within the hot junction skin.

(3) Similar to (2), with some biological shielding exterior to the radiator.

Type (3) was selected for more detailed analysis and design studies.

A computer code was developed to optimize generator designs. Development of another more general and versatile code for this purpose was begun.

Design efforts were directed to general arrangements of major generator components, and to some detail of components independent of generator configuration. The composition, form, and approximate quantity of the isotopic fuel were established. The requirements for biological shielding needed to permit safe handling of the power unit were calculated. Hazards associated with Curium-242 decay products and with the buildup of helium pressure in the fuel container were studied. A program to measure the Seebeck coefficient, electrical resistivity, and thermal conductivity of thermoelectric materials was initiated. 


\section{SUBTASK 5.8--CONCEPTUAL DESIGN OF 13-WATT THERMO- THERMOELECTRIC GENERATOR}

The objective of this program is to develop a conceptual design for a thermoelectric generator, fueled with Curium-242, to deliver 13 watts of electrical power for a space application. In order to achieve the minimum weight and maximum efficiency and reliability, several basic designs were examined. These designs were:

(1) A combination of several generators of the SNAP III type, with the hot junctions of the thermoelements in direct contact with the fuel container.

(2) A single generator of the SNAP III type, but enlarged so as to deliver 13 watts.

(3) A generator with spherical hot and cold junction surfaces, and with radiative transfer of heat from the fuel block to the hot junctions.

(4) A generator with radiative heat transfer, but with the thermoelements in contact with the lateral surfaces of two coaxial cylinders.

By means of analysis of heat transfer and radiation shielding, and by means of generator and component design studies, the optimum configuration was found to be of the radiative-cylindrical type. The weight of the generator, exclusive of voltage regulation equipment, is approximately 8.6 pounds, and the overall efficiency is $6.2 \%$.. Additional detailed design studies were undertaken to establish the configuration. A shielded shipping cask was partly designed.

Component design problems, especially the heat dump and the thermoelement contacts, were investigated. Hazards associated with the curium fuel were evaluated.

In December 1959, Jet Propulsion Laboratory indicated the desirability of application of the generator to a lunar impact mission, and recommendations for revision and extension of this program were prepared.

\section{TASK 6--FUEL TECHNOLOGY DEVELOPMENT}

This task covers the research, development, design and testing of radioisotope fuels. Included in this effort are the purification of 
americium, compacting of this fuel into aluminum slugs for irradiation, vaporization of the aluminum slugs after irradiation, purification of the curium, conversion to suitable fuel compounds for combination with matrux naterials, encapsulation in suitable containers for meeting the anticipated environment, and testing of the source capsules. In addition, shielding hazards, and handling and transportation requirements for all phases of processing will be evaluated.

A similar development effort for plutonium fuel forms is being conducted under this task (see MND-P-2176 Rev. 1).

The principal effort of this quarter was directed toward a parametric study of the radioisotopes suitable for isotopic power, the feasibility of processing the selected isotopes, and the selection of the procedures for purification. This parametric study is scheduled for completion during the second quarter, but a preliminary basis of operation was established for design of equipment during this period.

A survey of container materials suitable for maintaining the integrity of the capsule under abort conditions while capable of burnup during reentry from orbital flight has been initiated. 
Blank page 


\section{INTRODUCTION}

This is the first of three quarterly progress reports required by Contract $\mathrm{AT}(30-3) 217$ between The Martin Company and the AEC for fiscal 1960.

The whole SNAP program has been extensively reorganized under the current contract. The present organization is as follows.

TASK 1--INACTIVE

TASK 2--CLASSIFIED

TASK 3--NUCLEAR THERMOELECTRIC GENERATOR DEVELOPMENT (FORMERLY SNAP III)

TASK 4--CLASSIFIED

TASK 5 --ADVANCED TECHNOLOGY PROGRAM

SUBTASK 5.1 ADVANCED THERMOELECTRIC POWER SYSTEM

5.2 BASIC THERMONIC DEVELOPMENT

5.3 DEVELOPMENT OF LOW POWER THERMIONIC GENERATOR

5.4 (INACTIVE)

5.5 THERMOELECTRIC 2 to 5 WATT GENERATOR FOR SPACE USE

5.6 NUCLEAR 1.0 WATT POWER SUPPLY

5.7 100-WATT THERMOELECTRIC GENERATOR CONCEPTUAL DESIGN

5.8 13-WATT THERMOELECTRIC GENERATOR CONCEPTUAL DESIGN

TASK 6--FUEL TECHNOLOGY DEVELOPMENT

SUBTASK 6.1 GENERAL DEVELOPMENT AND MATERIAL REQUIREMENT

6.2 AMERICUM AND CURIUM RADIOISOTOPE PREPARATION PROCESSES 


\subsection{FUEL FORM}

\subsection{HAZARDS CRITERIA AND SHIELDING}

\subsection{RADIATION DAMAGE TESTING.}

Each task is separately administered. Tasks 3,5 and 6 are now unclassified. This report is the first unclassified techncal progress document published since the inception or the radioisotope power program. It covers work done on the unclassified tasks during October through December 1959. This period is the first contract quarter under the current contract and the twelfth project quarter of the total program.

For progress on Task 2, see MND-P-3009 (secret defense information). For the preceeding period see MND-P-3008 (secret defense information).

The principal efforts during the quarter centered on conditional or conceptual designs, applied research development and readings, and revisions to the program plan. 


\section{TASK 3--SNAP III GENERATOR*}

During the preceding quarter, a test generator failed after extended operational testing. The efforts of this quarter were directed toward analyzing the cause of the failure and revising the test specifications in line with the findings of this analysis.

\section{A. ANALYYSIS OF GENERATOR FAILURE}

No specific cause was found for the failure of the generator. However, the normal hot shoe temperature limits had probably been exceeded for a considerable period of time; this was indicated by substantial sublimation from the lead telluride elements. One element was broken in half but was conducting satisfactorily due to the spring pressure holding the two halves in contact.

Although the specific cause for failure was not found, it is believed that failure was due to general deterioration resulting from excessive sublimation and corrosion of the hot shoes which prevented element contact at the shoe face. This probably occurred at the P element junction since the $P$ element shows very little tendency to form a weld bond with the hot shoe. Figure 1 is an enlarged photograph of the hot shoe faces; it indicates that a good weld is obtained with the $N$ type element but little if any weld is present at the $\mathrm{P}$ element face. Figure 2 shows the general condition of two element assemblies as removed from the generator.

The previous quarterly report, MND-P-3008, states that the power output lead may have been open. This was not the case, however, since the lead proved to be an electrically intact circuit, although a break was indicated by the twisted junction of the wire ends.

New thermoelectric elements of standard design were obtained from the Minnesota Mining and Manufacturing Company. An attempt was made to machine these elements down to size to fit the test unit, with only partial success due to the extreme brittleness of the material. Only two of six elements remained intact after the machining operation. Due to severe deterioration of the mica insulation sleeves which cover the elements, new isomica sleeves were ordered from the Mica Insulator Company of Schenectady, New York.

At the end of this period, it appeared that the generator will be completely reassembled early next month and will be back on test by the end of the month.

*G. H. Storrs 


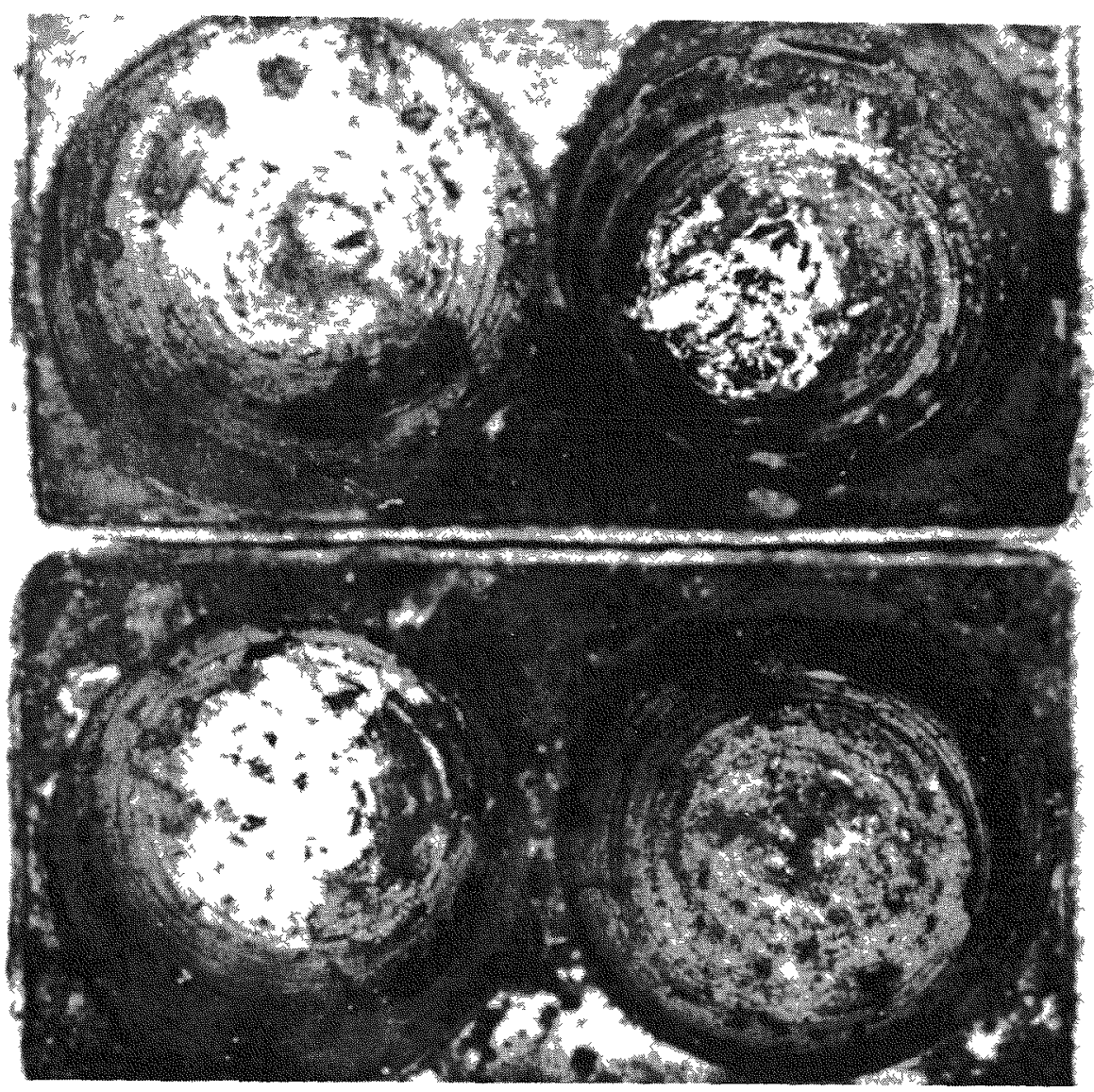

Fig. 1. Hot Shoe Faces 


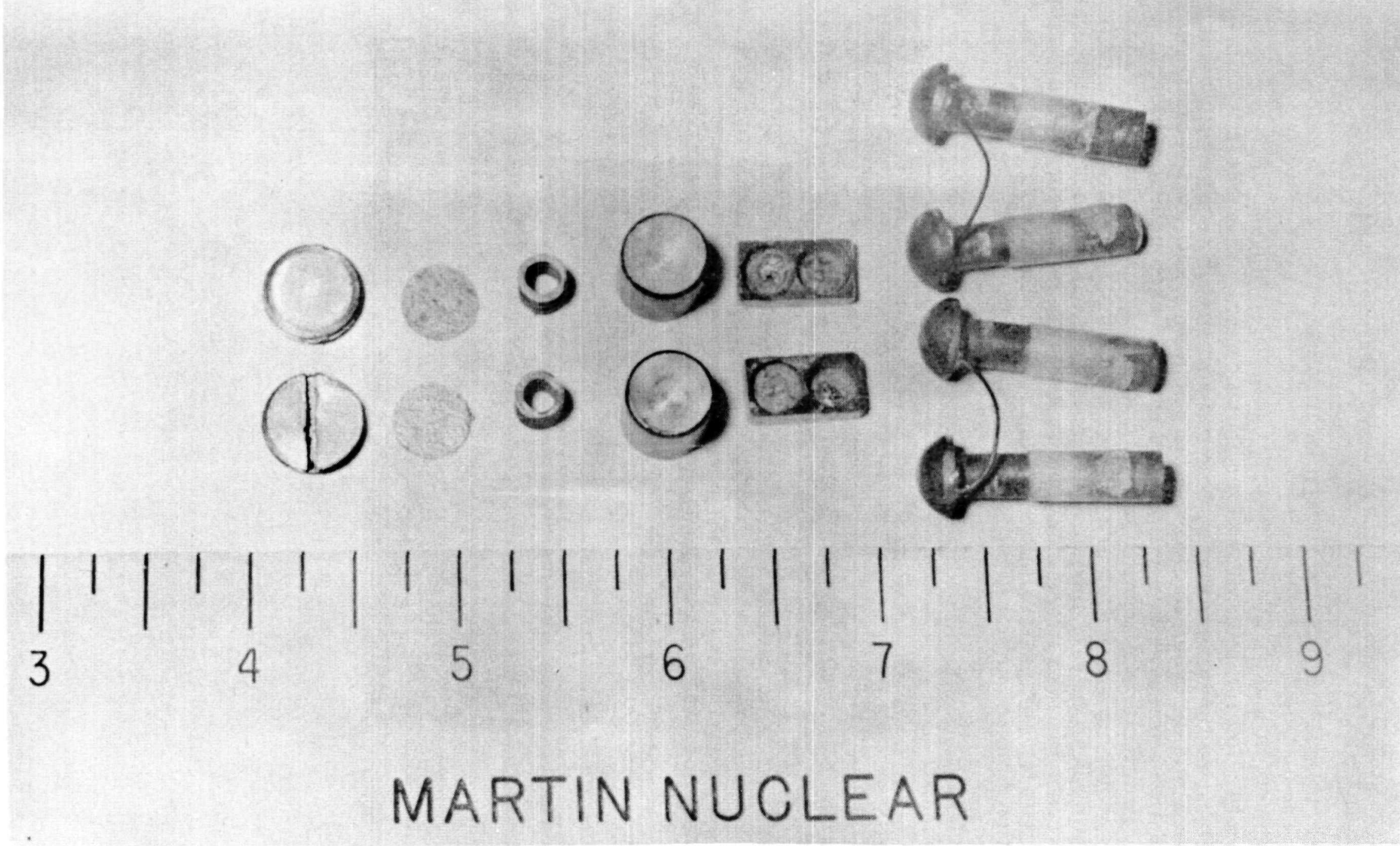

$\omega$

Fig. 2. Element Assemblies Removed from Generator 


\section{B. TEST REVISION}

A new test specification (MNET-JV-49) dated November 19, 1959 , was written for future testing of the thermoelectric generator. In this specification, there are two methods of evaluation. First, equilibrium or steady-state parametric conditions of power input, load resistance and heat sink temperature will be attained. At each equilibrium condition, power output, open circuit voltage, "short circuit current" and all generator temperatures are to be measured. The second method of evaluation is to quickly measure (instantaneous values) output voltage and current for load resistances from short circuit to open circuit, with the generator at equilibrium operating conditions under optimum load condition (maximum power output).

Under steady-state conditions, each generator will be operated and performance data obtained for power inputs ranging from maximum permissible power input to minimum useful power input. The five power inputs to be used are listed in Table 1. These power inputs will be determined with the generator under no load and when the hot junction temperature is stabilized for the internal gas environment specified.

TABLE 1

Maximum Hot Junction Temperatures Allowable Vs Internal Generator Pressures

\begin{tabular}{|c|c|c|c|}
\hline $\begin{array}{c}\text { Input Power } \\
\text { Level }\end{array}$ & $\begin{array}{c}\text { Gas at } 1 \text { Atmos } \\
\left({ }^{\circ} \mathrm{F}\right)\end{array}$ & Vacuum & P mm $\underset{\left({ }^{\circ} \mathrm{F}\right)}{\mathrm{Hg} \text { Pressure }}$ \\
\hline $\mathrm{P}_{\max }$ & 1100 & 900 & $T=900+(P / 760)(200)$ \\
\hline $\mathrm{P}_{2}$ & 1000 & 800 & $800+(P / 760)(200)$ \\
\hline$P_{3}$ & 900 & 700 & $700+(P / 760)(200)$ \\
\hline $\mathrm{P}_{4}$ & 800 & 600 & $600+(P / 760)(200)$ \\
\hline $\mathrm{P}_{\text {min }}$ & 700 & 500 & $500+(P / 760)(200)$ \\
\hline
\end{tabular}

Other Gas at Pressures $\left({ }^{\circ} \mathrm{F}\right)$ 
This procedure is designed to prevent inadvertent overheating of the hot junctions.

For each of the above power inputs, maximum equilibrium output current at "short circuit" (load resistance less than $0.10 \mathrm{ohm}$ ) will be obtained. Equilibrium conditions will also be obtained at nine load currents spaced approximately equally between zero and "short circuit" current for each of the five power input levels. Data as listed below will be taken to enable the determination of generator power output, generator power input, load resistance, internal resistance and various temperatures at each equilibrium power-load condition.

Output voltage

Output current

Input voltage

Input current

Hot junction temperature

Cold junction temperature

Shell temperature

Heater temperature (if available)

To determine the transient characteristics of the generator when stabilized at maximum power output for each of the predetermined power input levels, the output voltage and current will be obtained for three values (approximately equally spaced) between steady-state load and open circuit, and three values (approximately equally spaced) between steadystate load and "short circuit." 
Blank page 


\section{SUBTASK 5.1--ADVANCED THERMOELECTRIC POWER SYSTEM*}

The objective of this subtask is to evaluate and determine the performance characteristics of the electrically heated, 3-watt thermoelectric generator (SNAP IIIA) presently being fabricated by the New Products Engineering Department of Westinghouse. This generator includes a variable heat dump mechanism for thermal power flattening.

\section{A. STATUS OF THE WESTINGHOUSE GENERATOR}

Modifications were made to improve the structural integrity of the thermoelectric elements in the generator. The revised elements are larger than the original, so fewer elements are required. These revised elements were fabricated, the couples formed, and the generator assembled in the early part of December 1959.

During the calibration and demonstration runs, a leak developed in the valving mechanism of the variable heat dump system. Attempts were made to repair the valve, and the generator was delivered to Martin Nuclear for test. Examination disclosed that the valve was not satisfactory, so the generator was returned to Westinghouse for further repair, along with a request for an operating manual and a complete set of drawings. This was the status of the generator at the close of the period.

\section{B. DESCRIPTION OF THE WESTINGHOUSE SNAP IIIA GENERATOR}

The generator is as shown in Fig. 3. The generator weighs 10.18 pounds including the electrical heater, or approximately 7 pounds without the heater. The $N$ elements are lead telluride and the segmented $P$ elements consist of germanium telluride at the hot junction end of the element and zinc antimonide at the cold junction end of the element. The assembly of the generator is as shown in Fig. 4. As shown in Section A-A of Fig. 4, the generator consists of four sections located symmetrically around the cylindrical heat source; two thermoelectric ladders located 180 degrees apart, and two heat dump sections also located 180 degrees apart.

The generator was designed to utilize an isotopic heat source; it therefore has a heat dump mechanism for regulating the power output over the life of the generator, as the isotope power output decreases. The design life of the generator is one half-life of the isotopic heat source. Theoretically, the gas heat-dump mechanism should conduct away one-half of the power output from the isotope at the beginning of

\footnotetext{
*R. Harvey
} 


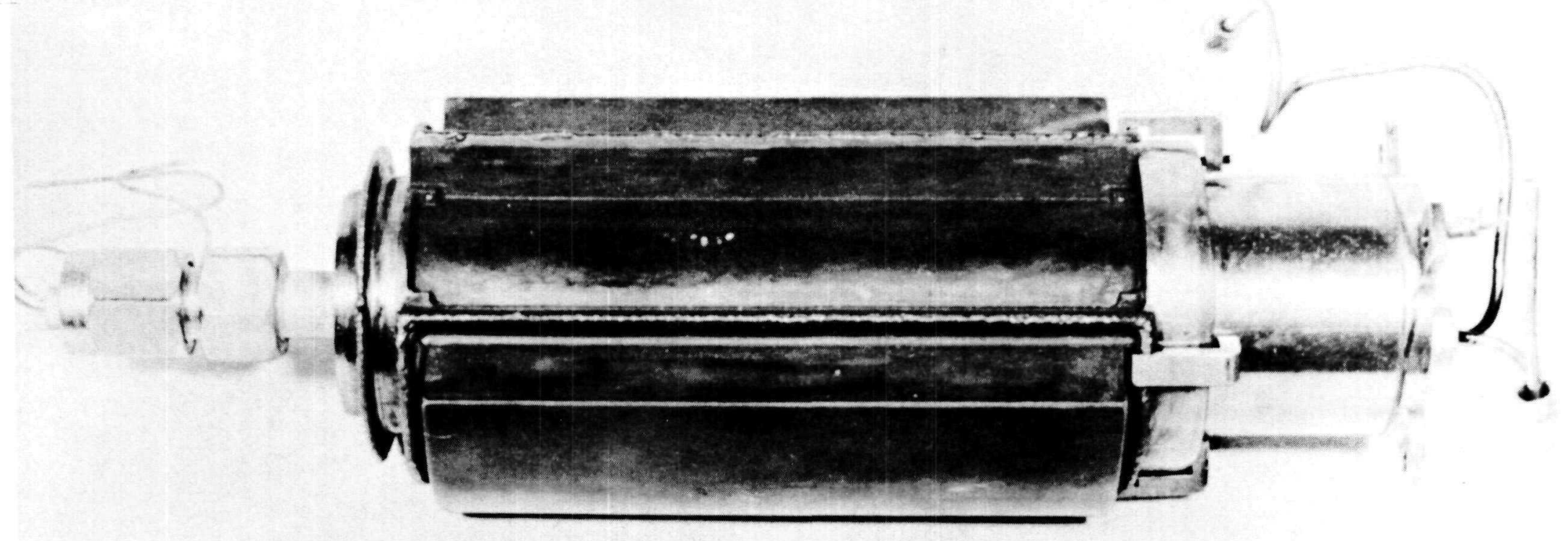

MARTIN NUCLEAR

Fig. 3. SNAP-III A Thermoelectric Generator 


\section{NOTES:}

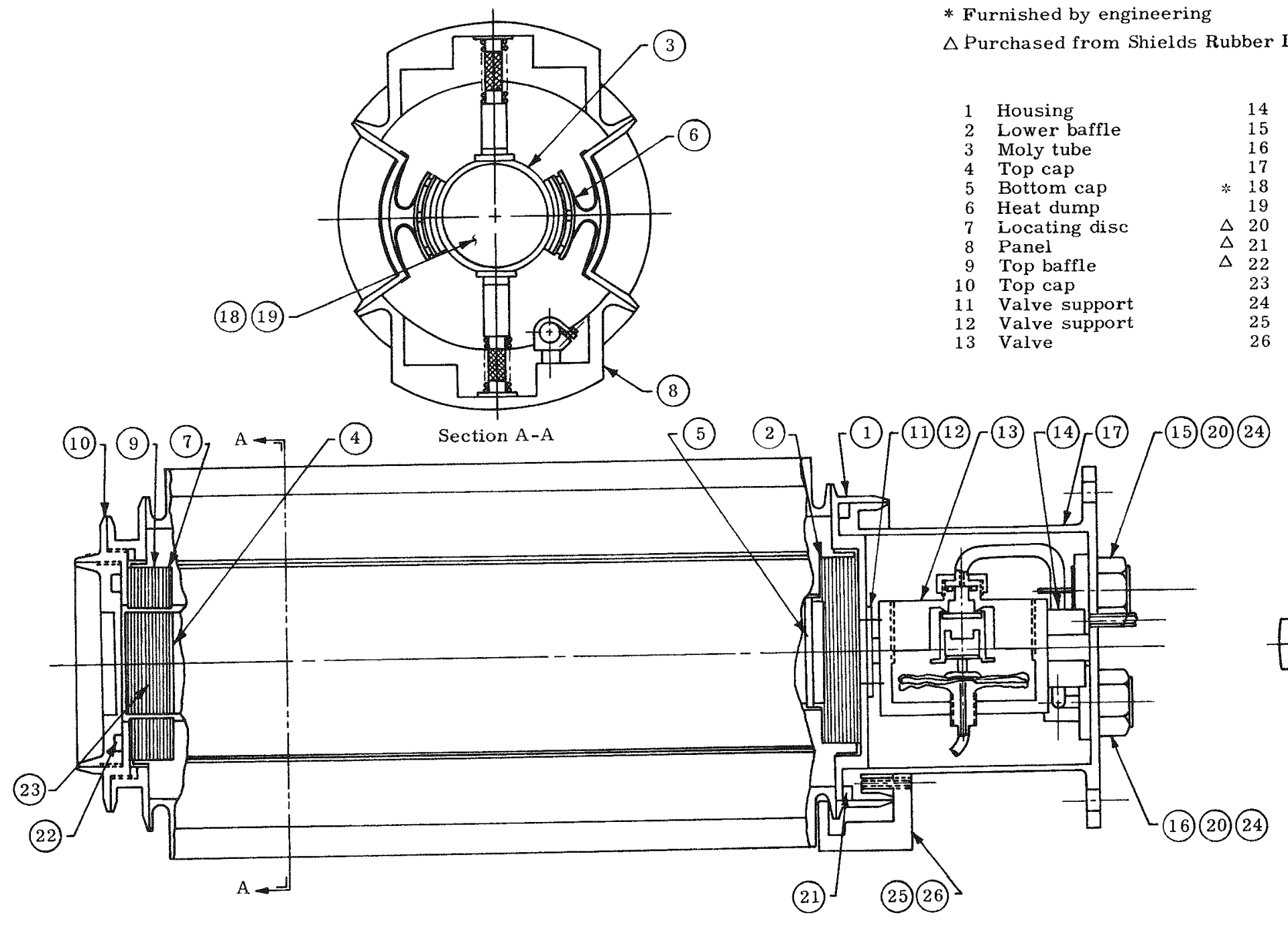

* Furnished by engineering

$\triangle$ Purchased from Shields Rubber Inc., Pittsburgh 22, Pa.

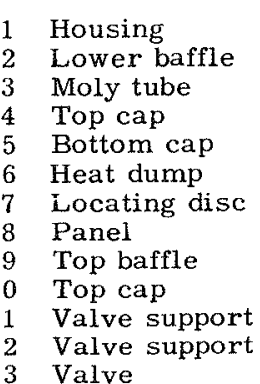

14 Porous Plug

15 Terminal seal

16 Exhaust sea

18 Valve can

18 Heat source

19 Test heat source

$\triangle 20$ "O" ring

$\Delta 21$ "O" ring

$\triangle 22$ "O" ring

24 Nuts--5/16--24 hex lock nut-SST

$25 \quad 112-40 \times 9 / 16$ SST set screw

Valve

26 Clamp

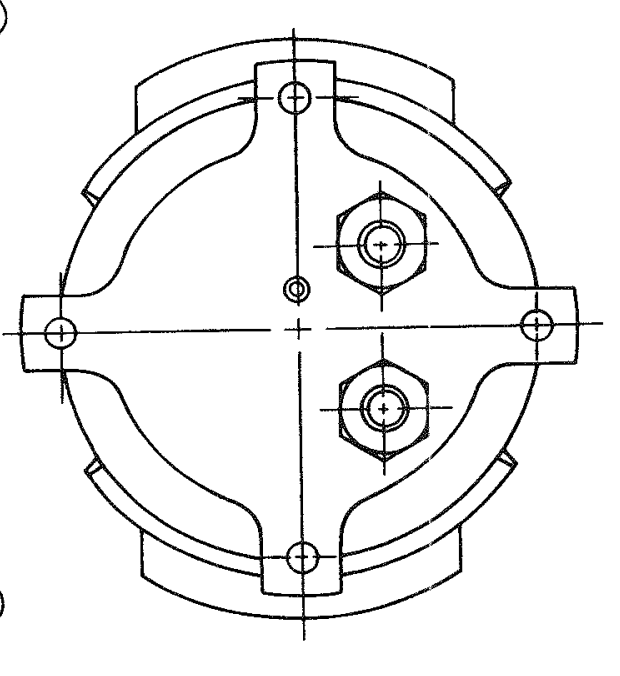

Fig. 4. SNAP III-A Thermoelectric Generator Assembly 
life, and no heat at the end of life. The generator must leak the gas in such a way as to match the exponential decay of the isotopic heat source over the half-life.

The heat dump mechanism operates on the thermal conductivitypressure relationship of gases. Argon is the gas chosen for this generator.

Table 2 lists data for the argon fill gas taken at the highest and lowest pressure points.

$$
\frac{\text { TABLE } 2 *}{\text { Performance Data }}
$$

$760 \mathrm{~mm}$

$$
\text { Argon Pressure }
$$

$0.07 \mathrm{~mm}$

$577^{\circ} \mathrm{C}$

$224^{\circ} \mathrm{C}$

$234^{\circ} \mathrm{C}$

482 milliohms

169 watts

1.78 watts

$0.7 \%$
Hot junction temperature

Cold junction temperature

Heat dump temperature

Internal resistance

Power input

Power output

Efficiency $544^{\circ} \mathrm{C}$

$172^{\circ} \mathrm{C}$

$175^{\circ} \mathrm{C}$

385 milliohms

91.5 watts

0.83 watts

$0.9 \%$

* Data reported by Westinghouse.

\section{PERFORMANCE EVALUATION PROGRAM}

A detailed test procedure for evaluating the Westinghouse thermoelectric generator was prepared. The tests are to consist of two parts, the electrical parametric tests and the power flattening tests.

The parametric tests are to determine generator power output, efficiency and junction temperatures as a function of power input, load resistance, internal gas pressure and external environment. 
The power flattening tests will be performed over a period simulating a half-life of the isotope, Po-210 in this case. Thermal power input to the generator is to be varied to simulate the decay of the isotopic heat source. The power output, internal gas pressure, and generator temperatures will be monitored. The load will be a matched load and the environment will be a vacuum with a black body receiver at a significantly low temperature.

The limiting operating conditions of the generator will be determined by taking data manually. These same data points will be reproduced on the automatic data-taking device to serve as a checkout of this device. Following the successful checkout of the automatic equipment, all other tests will be recorded automatically 24 hours a day, five days a week.

The test equipment was assembled. Construction of the vacuum bell jar base plate, on which the generator is to be mounted, was started. Passages through the base plate provide access for adjusting generator internal gas conditions, external environment, coolant flow and all necessary electrical functions (heat leads, thermocouple leads, etc。) The bell jar system was approximately $70 \%$ complete before work was stopped due to the inoperative condition of the generator. 
Blank page 


\section{SUBTASK 5.2--BASIC THERMIONIC DEVELOPMENT*}

The objective of this subtask is to advance the technology of thermionic converters, specifically, to increase the efficiency of thermionic generators by developing better emitter and collector materials and by reducing heat losses. This work is being performed by Thermo Electron Engineering Corporation.

For convenience, the work has been organized as follows:
A. Cesium Diodes
B. Effect of Cesium on Materials
C. Electrical Heaters
D. Diffusion of Gases Through Metals
$E_{\text {。 }}$ Work Function Study.

\section{A. CESIUM DIODES}

Work has been performed on four different types of cesium diodes during this reporting period. These are:

(1) Close-spaced cesium diodes

(2) Wire-emitter cesium diodes

(3) Ribbon-emitter cesium diodes

(4) Electron-heated cesium diodes.

\section{Close-Spaced Cesium Diodes}

A simple diode was constructed to study the effect of cesium on the space charge barrier between a closely spaced emitter and collector. The diode consisted of an impregnated emitter and a pure molybdenum collector. Both electrodes were 0.748 inch in diameter. The electrodes used in the prototype are of the same diameter. This size was chosen in order to observe directly the effect of cesium on the cathode performance. Sapphire spheres separate the emitting and collecting surfaces, giving a spacing of approximately $0.001 \mathrm{inch}$. The collector was cooled by radiation from its side. The emitter was heated by a simple spiral-wound tungsten heater. The emitter was shielded

\footnotetext{
* $R$, Harvey
} 
around the periphery to reduce radiation losses. After the tube was baked out for several hours, the cathodes were activated and the getter was fired before sealing off the tube from the vacuum pump.

The cesium source for this tube was contained in eight nickel capsules and consisted of a mixture of cesium chromate and silicon. The pure cesium was formed by heating these capsules in a radiofrequency furnace. The section of the tube containing the cesium was immersed in a variable temperature oil bath. The top part of the tube and the presses were maintained at a higher temperature by means of a heater tape and a heater lamp. This was done to avoid condensation of cesium on the cathodes and supporting structure.

Tests were made with and without cesium at very high emitter temperatures and, in the former case, at very high cesium bath temperatures. Unfortunately, sufficient cesium was not available at the highest cesium bath temperatures. It was determined that the space charge reduction due to cesium was small in this case, since almost as much output power had been obtained without cesium.

One reason for this appears to be the fact that the smallest mean free path of the cesium atom, determined by the highest cesium bath temperature, was several times larger than the emitter-collector spacing. This resulted in a decrease in cesium pressure in the interelectrode space, below that indicated by the bath temperature. This pressure gradient indicates that we cannot accurately interpret the experimental results. However, the results obtained from this cesium tube did indicate that there was a lesser pressure in the interelectrode space than in the rest of the tube. Two remedies could be tried. Either the spacing between the emitter and the collector might be increased, or higher cesium bath temperatures might be attained. The mean free path would be less than the spacing used in either case.

The tests were terminated when a crack developed in one of the presses.

\section{Wire-Emitter Cesium Diodes}

In November, a cesium diode was designed with a tungsten wire emitter, a tungsten wire ion source and a tubular silver collector. Silver guard-rings were used to prevent collector end effects. Figure 5 is a drawing of the tube.

The assembly for the wire-emitter cesium diode was completed and tested in a bell jar vacuum system. With the small diameter of the silver collector tube, it was extremely difficult to mount two 


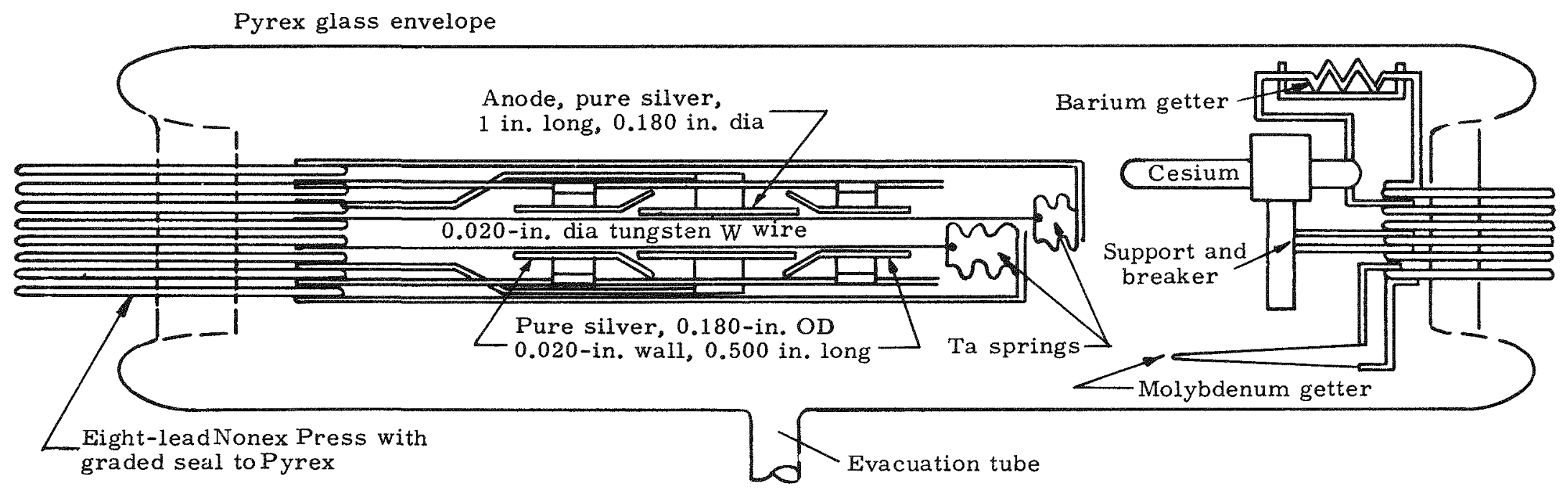

Fig. 5. High Temperature Wire-Emitter Cesium Diode 
tungsten emitter wires with springs through the tube. Although the construction was accomplished three times, with modifications, when the wires were heated to operating temperature (about $2000^{\circ} \mathrm{K}$ ) they shorted either to each other or to the silver tungsten wire emitter down the center of the collector.

Springs of $0.020-$ in. OD are wound from the same wire at each end for tension. Although the springs kept the wire straight and presumably in tension, the hotter spring (at the top of the tube away from the press) became longer and the colder spring became shorter; this permanent deformation is undesirable. Future springs will be made of larger diameter wire (0.025 in.).

The filament was operated for about one hour at 2000 to $2500^{\circ} \mathrm{K}$ to determine effects of time and temperature on the structure. The silver tube became so hot that it melted at its center, despite care in construction to provide conduction paths away from the collector and to provide a large radiating area. The collector $O D$ and supports were purposely roughened with emery cloth to increase emissivity. Approximately 300 watts input were necessary to reach $2500^{\circ} \mathrm{K}$ emitter temperature.

\section{Ribbon-Emitter Cesium Diodes}

A resistance-heated ribbon emitter is being investigated to determine the effects of cesium on output power and operating characteristics of a close-spaced diode. Although the design of the device was not yet final, a preliminary configuration for the emitter structure was built and tested.

The test apparatus consists of a 0.0013 -in. tungsten ribbon, 1 $\mathrm{cm}$ wide except in the $4-\mathrm{mm}$-wide test section, supported by two 0.010 -in. tantalum lead strips. The test section is an emitting area, $2 \mathrm{~cm}$ long, supported by two 0,040-in. tantalum wires. The ribbon at the extremities of the test section is bent in several places to produce a spring effect. It is then spotwelded to the $0.010-\mathrm{in}$. tantalum sheet. This is shown in Fig. 6.

The emitter structure was tested in $10^{-6} \mathrm{~mm} \mathrm{Hg}$ vacuum under a glass bell jar. All temperatures were measured with an optical pyrometer and corrected for tungsten emissivity. Initially, the center of the emitting section reached a temperature higher than the area closest to the $0.040-$ in. supports as the element was electrically resistance-heated. 


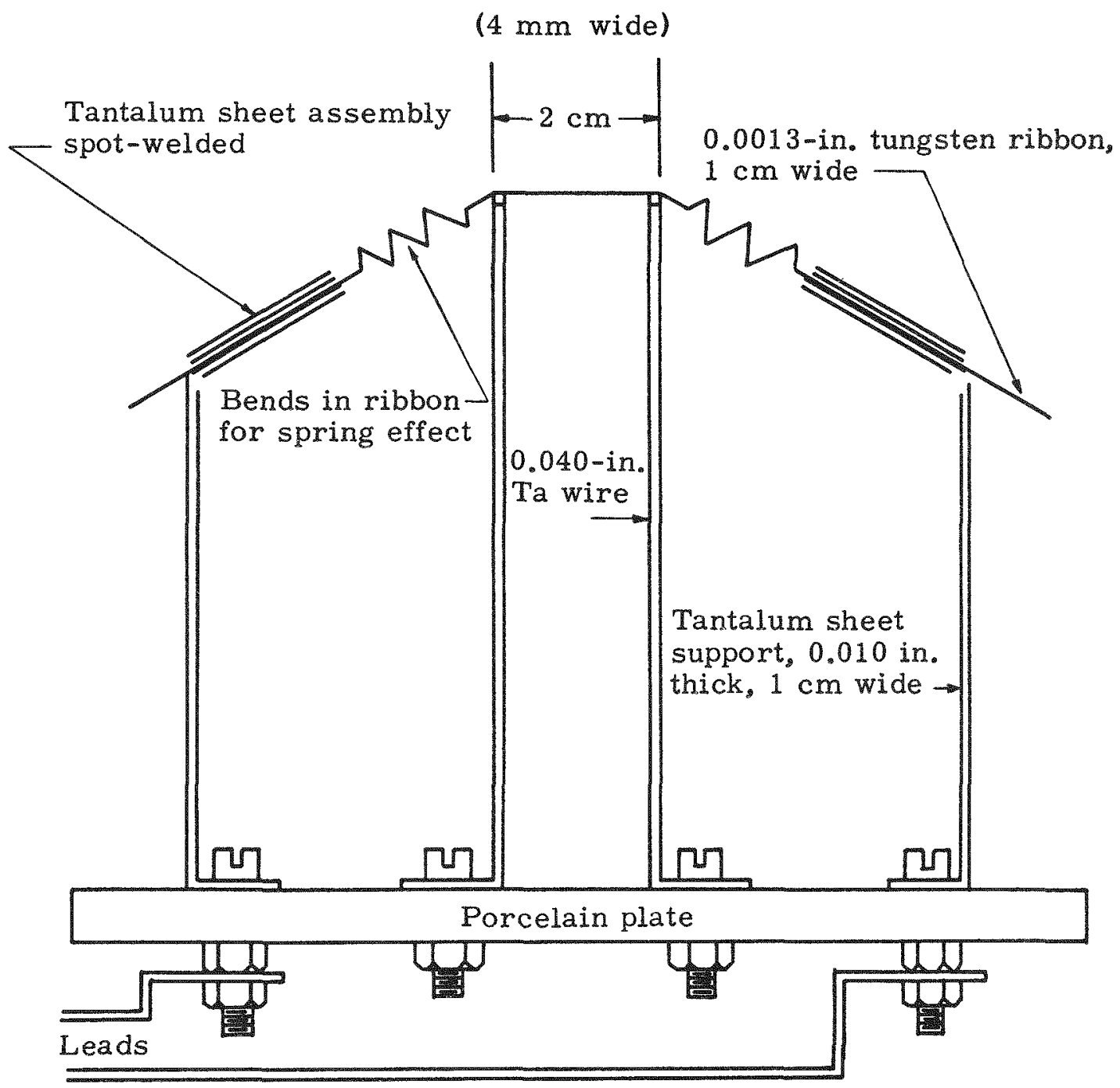

Fig. 6. Ribbon Filament Test Apparatus 
As the $I^{2} R$ heat input was increased, the high center temperature extended throughout the entire test section of the ribbon, until a uniform $1500^{\circ} \mathrm{K}$ was obtained. A further increase in the heat input to 272 watts increased the temperature of the emitting area to a uniform $2400^{\circ} \mathrm{K}$. The spring area between the $0.010-i n$. tantalum sheet support and the $0.040-\mathrm{in}$. tantalum wire was at $1370^{\circ} \mathrm{K}$. The temperature gradient between the $2400^{\circ} \mathrm{K}$ test section and the $1370^{\circ} \mathrm{K}$ spring area extended only over a ribbon length of $1 \mathrm{~mm}$. The emitting area remained flat, even through cyclic changes in temperature. The tantalum supports never became visibly hot.

The results of this experiment offered an encouraging indication that tungsten ribbon might be used for the emitting element in a cesium diode. The filament did not deform or heat nonuniformly; therefore, it is suitable for use in designs incorporating small emitter-collector separations.

A device similar to the test apparatus was then constructed (see Fig。 7). On each side of the ribbon emitter, a collector of 0.010 -inch thick tantalum sheet was supported by ceramic washers 0.027 inch thick. This device operated very successfully, except for some overheating of the collector. The emitter reached $2500^{\circ} \mathrm{K}$ and the collector at this temperature was at about $1500^{\circ} \mathrm{K}$. The emitter temperature appeared quite uniform over its length (above $1600^{\circ} \mathrm{K}$ according to measurements made with an optical pyrometer). The support structure did not become excessively hot。

Because of the structural success of this device, it was decided that the tube should be rebuilt with a revised collector (see Fig. 8). Flat plates of molybdenum 0.020 inch thick and $3 \mathrm{~cm}$ wide were substituted. The increased thickness and conductivity should reduce the high localized temperature just above the emitter and allow the collectors to reach an evenly distributed, decreased temperature. The press has been sealed in an envelope, with an arm for a cesium tube. This envelope is now being baked out prior to sealoff.

\section{Electron - Heated Cesium Diodes}

Certain basic engineering designs and methods of operation have been developed for an electron-heated cesium diode. The unit consists of two chambers: one chamber contains the cesium diode, which is sealed, and the second chamber, which houses the electron gun, is continuously pumped. A method for the controlled heating and cooling of the tube envelope, in order to obtain a desired cesium condensation temperature, has been developed. Envelope materials, leadthroughs and ceramic insulators have also been investigated. 


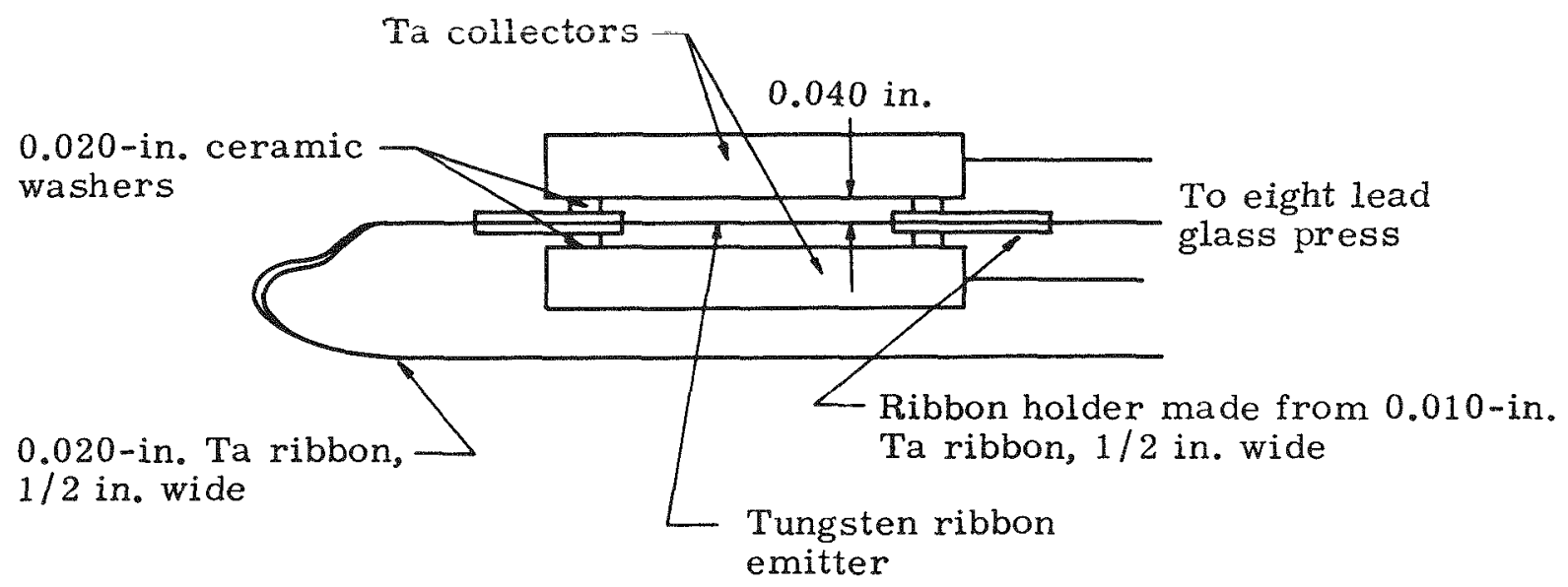

a. Side View

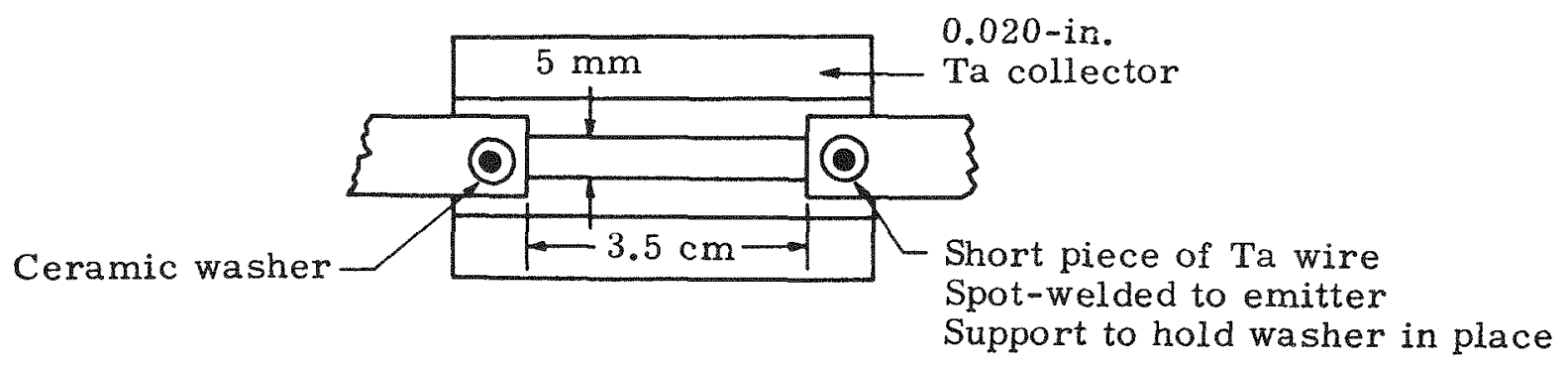

b. Top View (with top collector removed)

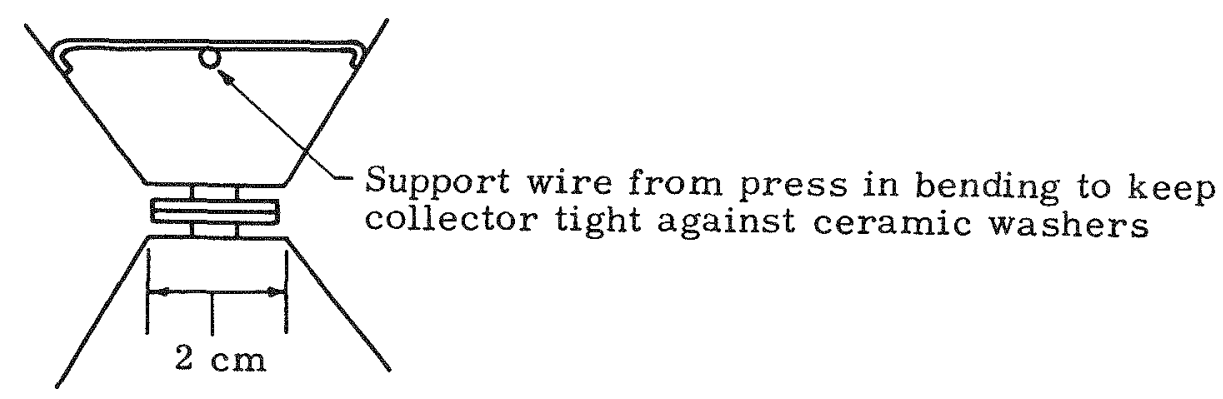

c. End View

Fig. 7. High Temperature $I^{2} \mathrm{R}-$ Heated Ribbon Emitter 


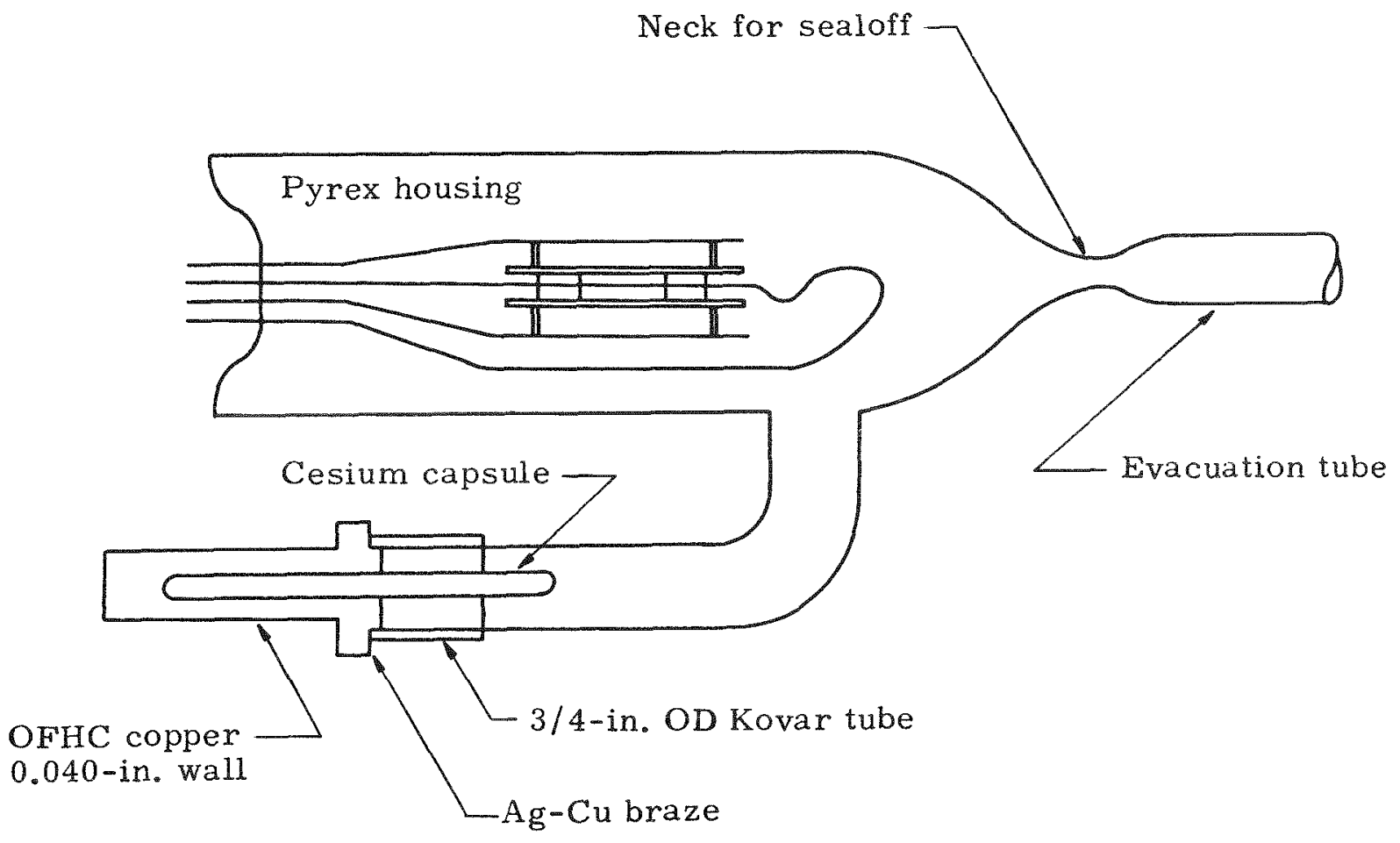

Fig. 8. Modified High Temperature $I^{2}$ R-Heated Ribbon Emitter Assembly 
To study the preliminary problems involved in electron heating, a test assembly was constructed as shown in Fig. 9. In the test apparatus, a 0.030 -inch tungsten filament and the tungsten impregnated emitter are supported by molybdenum posts. The three posts are firmly held by a porcelain plate. A bell jar covers the entire unit and is evacuated to a pressure of $10^{-6} \mathrm{~mm} \mathrm{Hg}$ 。 To supply power to the apparatus, a 600 -volt, 500-ma unit was designed and constructed.

When the filament current and the electron accelerating potential were adjusted to a maximum value, the temperature of the tungsten impregnated emitter was $1720^{\circ} \mathrm{K}$. The accelerating potential was 420 volts at 520 milliamps, yielding a heating power of 218 watts. The temperature obtained was uniform over the emitter. No significant problems were encountered.

Higher emitter temperatures are desired, however. To accomplish this, a 3000-volt, 500-ma power supply and a magnetic amplifier for filament current regulation are being constructed.

In support of this effort, an investigation of construction techniques, assembly of information on standard parts, a preliminary sketch and a partial assembly drawing were accomplished. No new hardware has been constructed. Some parts (tubing, leadthroughs) have been ordered. The design is crystallizing and a list of steps in the design and testing of parts of this device has been drawn up. Calculations of heat losses, thermal expansions, and mechanical strength are now being made.

\section{B. EFFECT OF CESIUM ON MATERIALS}

A literature search was undertaken to determine the corrosive and alloying effects of cesium on various materials at high and low temperatures. Tubes containing cesium were also carefully investigated for the effects of cesium on ceramics, metals, presses, brazes and welds.

Cesium corrosion may take place by several fairly common mechanisms. One is a uniform solution attack on solid surfaces by the cesium corrodent in its liquid state. Others are direct alloying, intergranular penetration and corrosion by contaminants. A literature search seemed the most expedient method of determining these effects of cesium on metals.

In addition, two vacuum tubes were constructed to determine the extent of cesium attack on certain metals. The tubes were identical except for the sample materials included in each. One contained OFHC copper, pure tantalum, tungsten and molybdenum; the second contained Type "A" nickel, an iron-nickel alloy, Kovar and pure silver. Figure 10 is a drawing of the test setup. 


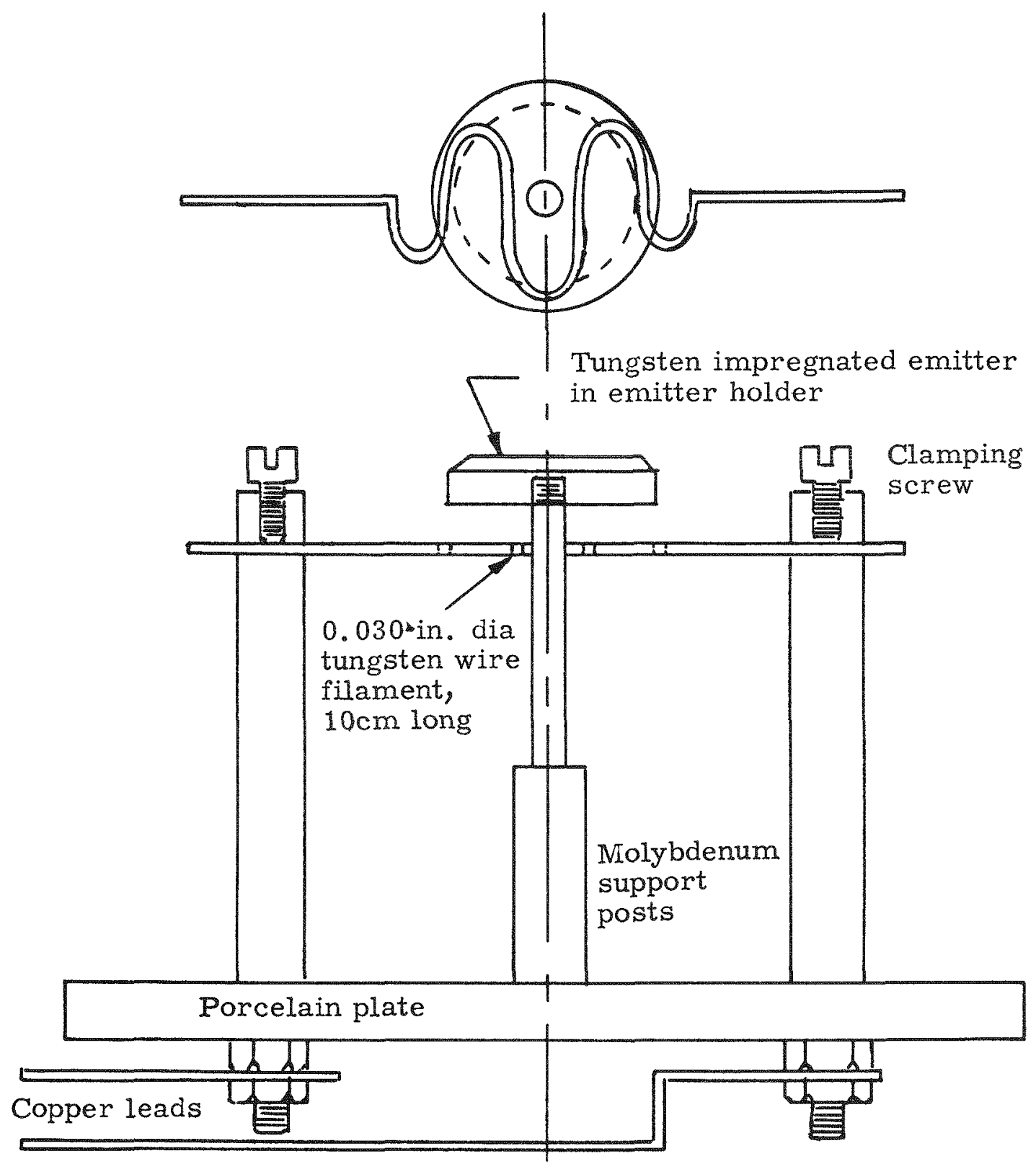

Fig. 9. Electron Heater Test Apparatus 


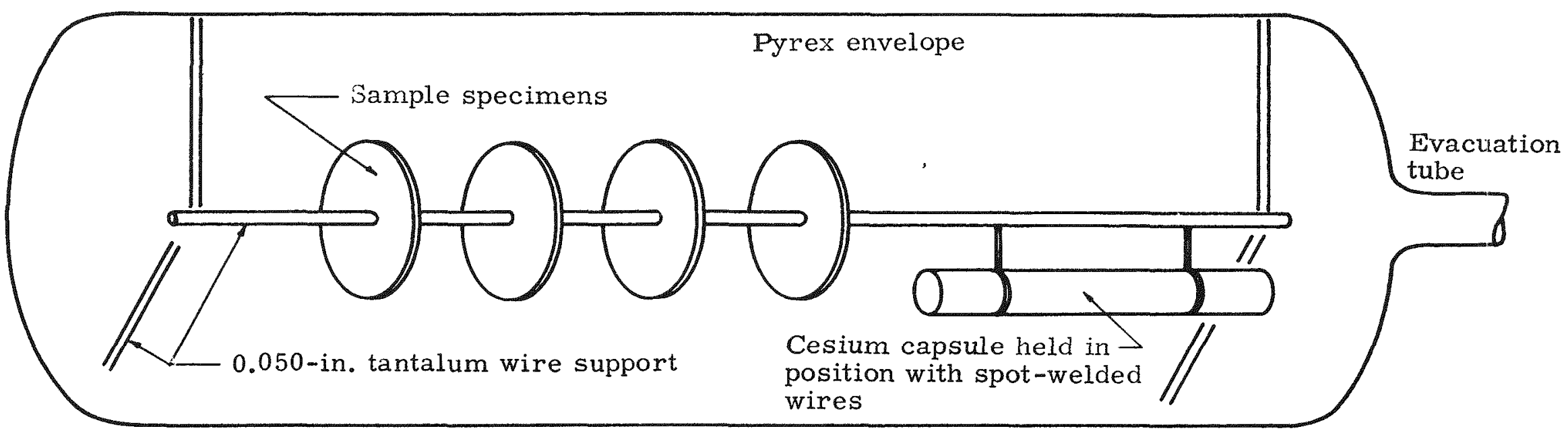


The iron-nickel material, Driver-Harris No. 142 , is a $41.5 \%$ nickel$58.5 \%$ iron alloy. Kovar is a $29 \%$ nickel $-17 \%$ cobalt $-54 \%$ iron alloy. These metals match the thermal expansion of certain glasses and ceramics. Attack on these materials is being investigated since they will be used for prototype casings and for metal-ceramic and metal-glass seals. Tantalum, tungsten, nickel and molybdenum are being tested because of their use as either emitter and collector materials or as structural supports where high operating temperatures exist. Copper and silver will be used as braze materials.

To determine the extent of cesium corrosion on metals, the pyrexenvelope tube containing samples of tungsten, copper, molybdenum, and tantalum was evacuated on a glass system, baked at a temperature of $720^{\circ} \mathrm{F}$ for two hours and sealed at a pressure of $1 \times 10^{-6} \mathrm{~mm} \mathrm{Hg}$.

The cesium capsule was opened by induction heating the support wires which encircle the capsule until the wires were sufficiently heated to locally melt the glass cesium container. The cesium was also heated by the RF furnace, which produced sufficient vapor pressure to force the cesium out through the soft glass.

With the sample metals exposed to the cesium corrodent, the tube was baked in an oven at $425^{\circ} \mathrm{C}$ for 118 hours. At the end of this period, no liquid cesium remained in the envelope. The pyrex, however, was a light yellow color. When the tube was opened, the yellow film readily flaked off the glass, leaving an etched surface on the pyrex. Preliminary analysis indicated a reaction took place between the cesium and the pyrex at $425^{\circ} \mathrm{C}$. Samples of the yellowish film were sent to an outside firm to be chemically and spectrographically analyzed.

No corrosive effects of cesium on the metal samples were evident. The results, however, are somewhat inconclusive since it is not known how long cesium vapor was present in the tube before the reaction which produced the yellow flakes consumed all the cesium.

A second tube has been constructed with a pyrex envelope housing samples of silver, Kovar, Driver-Harris alloy and nickel.

A third tube will be constructed as shown in Fig. 11. This tube will not employ a glass envelope and will permit higher temperature exposure.

\section{ELECTRICAL HEA TERS}

Two types of heaters were investigated. 


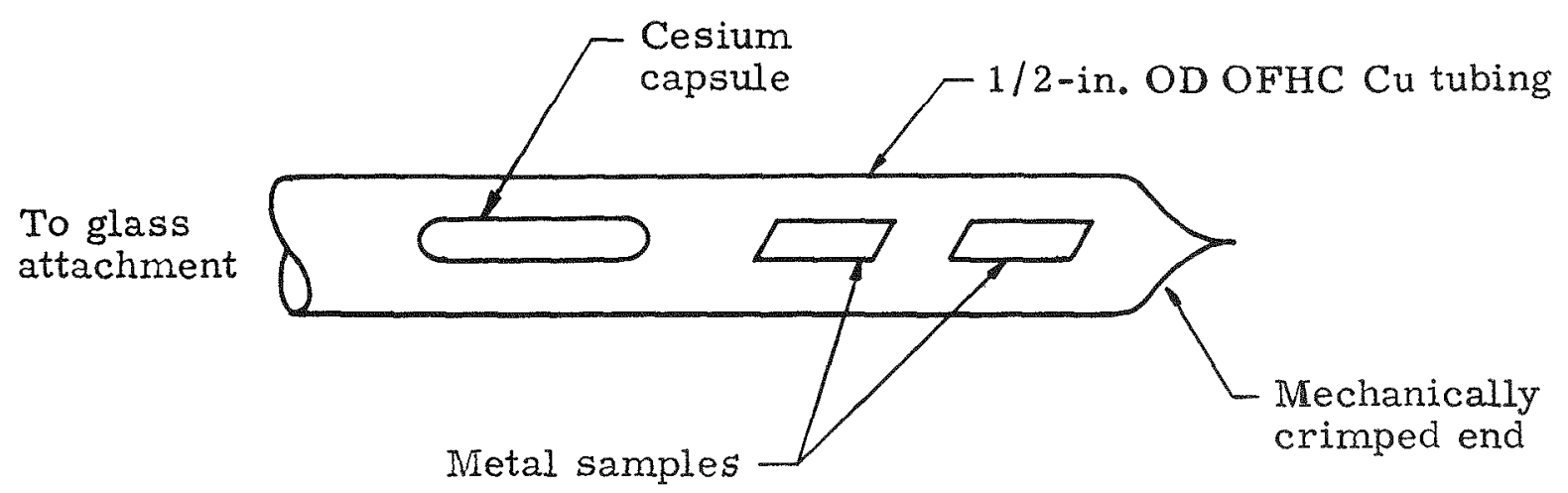

Fig. 11a. Apparatus to Determine the Effect of Cesium on Certain Materials

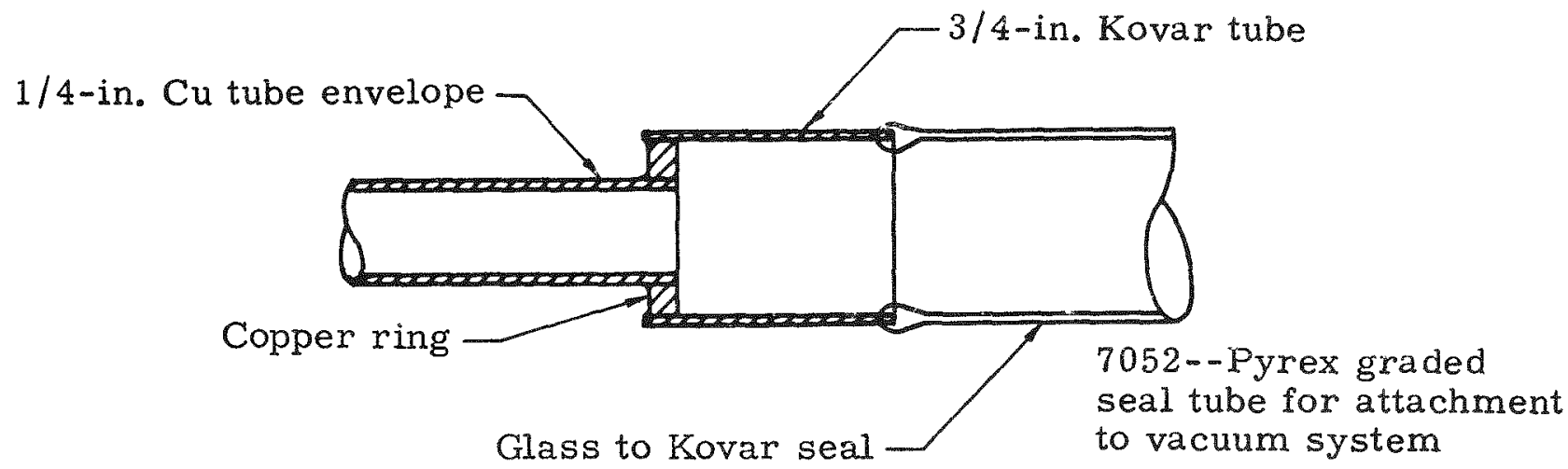

Fig. 11b. Detail of Cu to Glass Attachment 
The first type has the heating wire fitted into helical grooves cut in a ceramic cylinder. The second design involves metallizing a ceramic cylinder with molybdenum and making a helical cut in the periphery of the metallized cylinder. The metallizing work was done by an outside facility and the cutting and grinding have been done at the Thermo Electron Engineering Corporation. Tests made to date show that this metallized heater is the most promising from the standpoint of ruggedness and reliability。

The design features required of a long-life, reliable heater element include durability and stability in thermal shock and vibration. The heater should also be simple to make and install, and the geometry should be adaptable to various configurations. Heater designs have generally incorporated spiraled tungsten wire, wound on ceramic mandrels. However, other approaches, such as the metallized ceramics, are being considered.

\section{Wire-Wound Heater}

The heater element was made by winding 0.012 -in. diameter tungsten wire on 0.010 -in. molybdenum wire (see Fig. 12). When the tungsten had been annealed, the molybdenum was either chemically dissolved or mechanically removed, so that a tungsten coil remained. A solid cylindrical mandrel was diamond-ground from $94 \%$ pure, dense aluminum oxide and provided with double grooves for supporting the heater wire. Four holes were drilled ultrasonically for the leads.

The heater assembly was tested under a bell jar on a 3 -inch vacuum system. The mandrel rested on ceramic washers in a shallow molybdenum cup. No heat shielding was provided。

The system was evacuated to $10^{-6} \mathrm{~mm} \mathrm{Hg}$ and slowly increasing current was passed through the heater wire. At 45 volts, 2.75 amps, the wire temperature was a uniform $1900^{\circ} \mathrm{F}$ 。As the power was increased to 65 volts and 3.75 amps, the wire temperature reached $2500^{\circ} \mathrm{F}$ 。 The temperature of the ceramic read $1450^{\circ} \mathrm{F}$. Even though the heater wire was wrapped tightly on the ceramic, conduction between the wire and the ceramic apparently was poor, otherwise higher ceramic temperatures would have been observed.

The molybdenum leads should remain cool when heat is supplied to the tungsten filament to prevent embrittlement. Throughout the test, the leads remained at a sufficiently low temperature, Thus, the heat transfer design of the leads is judged to be satisfactory. 
Heater wire:

0.012 tungsten wire wound

on 0.010 molybdenum wire

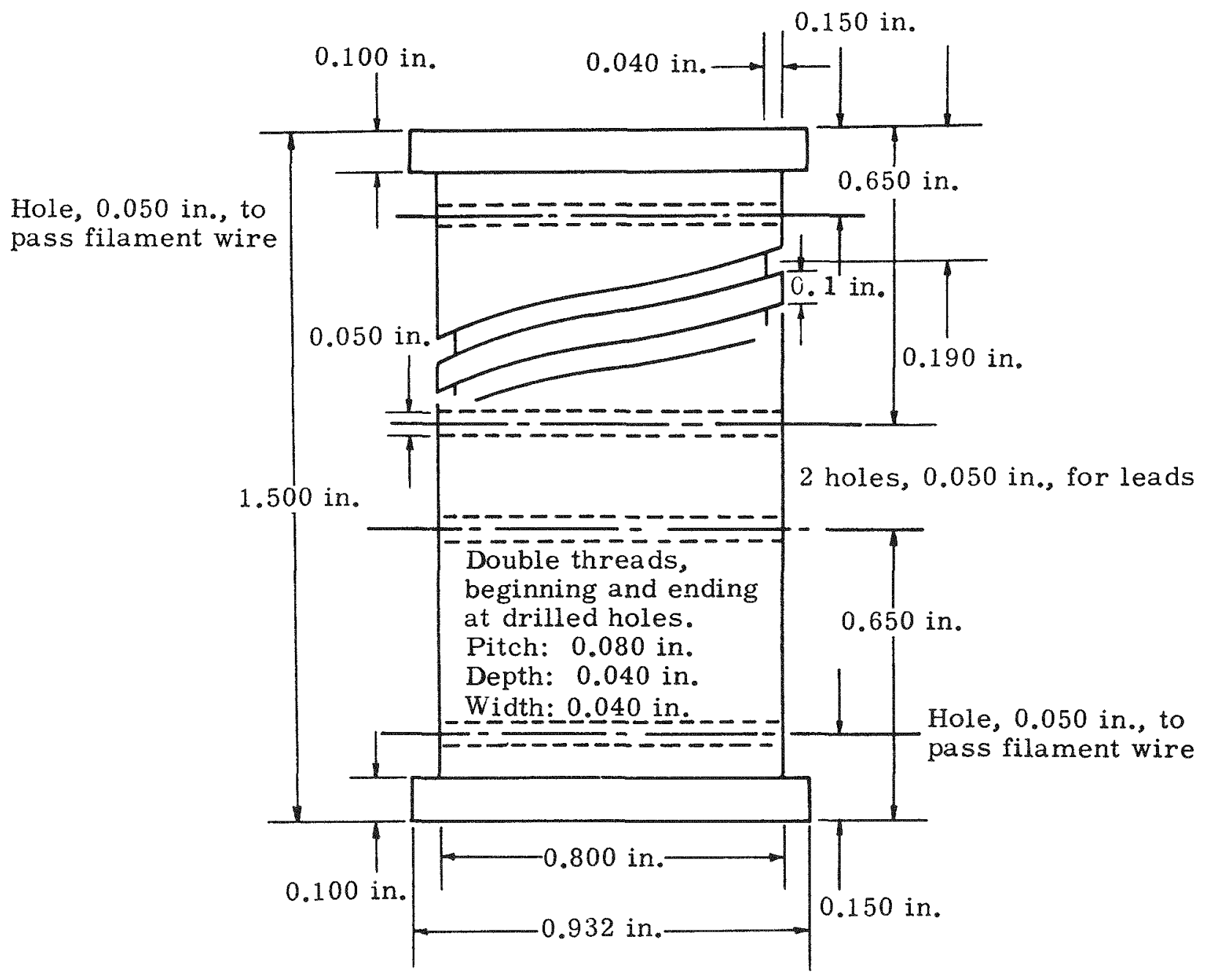

Not to scale

Note: Ends must be flat and parallel to \pm 0.003

Tolerances: Dec.: $\neq 0.005$

Fig. 12. Wire-Wound Heater 
Further testing of this heater unit will fllow. A 0.010-in. molybdenum sheet will be tightly wrapped around the ceramic mandrel. Conduction and radiation from the heater will raise the temperature of the cylindrical shell to a level high enough to provide the required heat source for cesium diode operation.

\section{Metallized Ceramic Heater}

The metallized ceramic heater is a unit made from a pure aluminum oxide tubing of $0.962-$ in. OD, 0.695-in. ID and 0.625 -in. length. The exterior walls of the cylinder and its ends are metallized with a $0.003-i n$. to 0.005 -in. coating of $94 \%$ molybdenum and $6 \%$ titanium. This heater is shown in the sketch below.

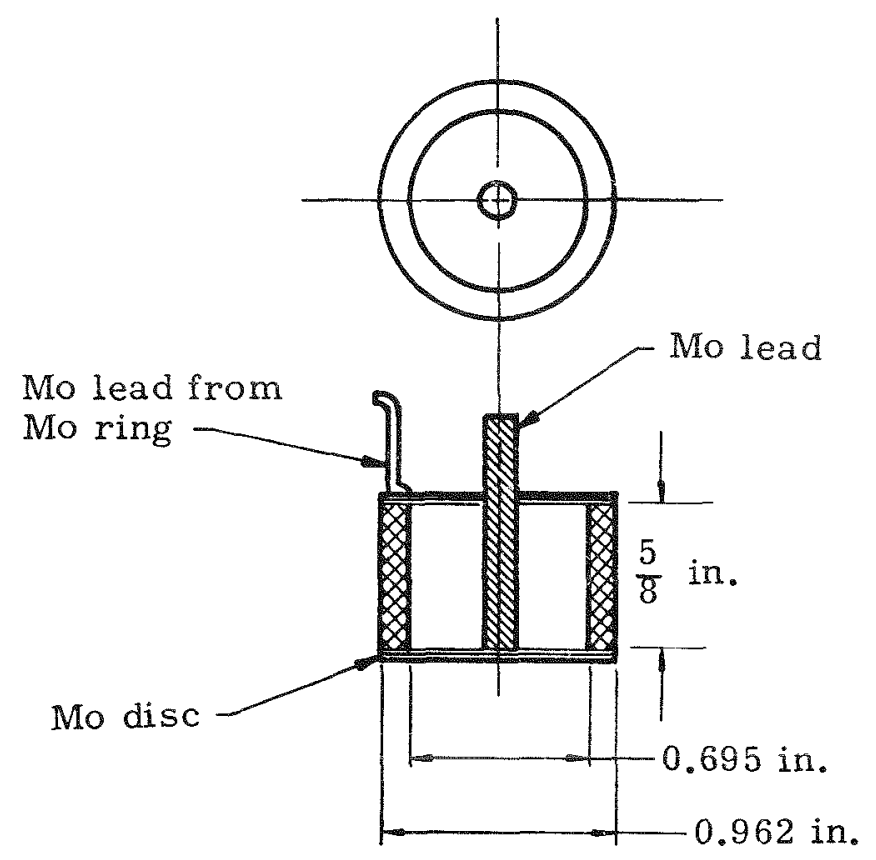

Metallized Ceramic Heater

The metallized ceramic periphery is diamond-ground to produce a metal helix approximately $0.030 \mathrm{in.}$ wide. Contacts for electrical leads are attached by brazing a molybdenum disk to one end of the ceramic tube and a molybdenum ring to the other end. To the disk is brazed a molybdenum rod which extends through the ceramic tube and serves as a lead. A lead wire is welded to the ring. 
Since the heater is to be operated between 1200 and $1300^{\circ} \mathrm{C}$ in vacuum, and since the aluminum oxide will soften at temperatures above $1400^{\circ} \mathrm{C}$, the braze material must melt and flow in the 1300 to $1400^{\circ} \mathrm{C}$ temperature range. At temperatures below $1300^{\circ} \mathrm{C}$, the braze must exhibit a vapor pressure less than $10^{-6} \mathrm{~mm} \mathrm{Hg}$. Brazes offering suitable characteristics are now being studied and evaluated.

A sintering method for joining the molybdenum pieces to the metallized ceramic is being considered as an alternate to the brazing process. The method involves sintering molybdenum powder at a relatively low temperature. The molybdenum disk and ring would be pressed firmly against the metallized ceramic by a sufficiently heavy weight placed on top of the assembly. The entire unit would then be brought to a temperature of $1400^{\circ} \mathrm{C}$ for $1 / 2$ to 3 hours in a $20^{\circ} \mathrm{C}$ to $40^{\circ} \mathrm{C}$ saturated mixture of pure hydrogen and water vapor. A tight bond should then result.

The interest in developing this heater design stems from the need for a rugged, reliable and easily mountable unit with long operating life. This assembly, when installed in thermionic devices, should meet vibration and shock requirements. If leads can readily be attached, this heater should meet all the specifications.

\section{DIFFUSION OF GASES THROUGH METALS}

To develop any device designed to operate in a vacuum, the phenomena of absorption of gases by metals and diffusion of gases through metals must be thoroughly understood. Absorption or solution of gases in metals is closely related to the process of gas diffusion through metals. Therefore, the basic phenomena can be studied by investigating only diffusion. Forturiately, diffusion of gas through metals is readily measured by laboratory analysis. Hydrogen exhibits the most advantageous characteristics for a gas to determine the porosity of a medium and the rate of diffusion. Because of this, diffusion of $\mathrm{H}_{2}$ through metals was investigated.

A literature search was undertaken on the diffusion of various gases through metals. Hydrogen generally has the highest rate of diffusion through metals and the rate varies with the temperature of the metal. The literature is being critically reviewed to compile all the available data on the diffusion rates of hydrogen through metals. Temperature versus rate data will be plotted. Diffusion of hydrogen from a high pressure side at $760 \mathrm{~mm}$ of $\mathrm{Hg}$ to a low pressure side below 1 micron of $\mathrm{Hg}$ in a clean sample will be the standards of measurement. The methods for calculating the diffusion rates of hydrogen and other gases through metals will be investigated. A bibliography of pertinent references will be compiled. 
If the literature search yields insufficient information on particular materials applicable to the proposed prototypes, tests of specific metals will be undertaken.

\section{E. WORK FUNCTION TESTS}

This section describes tests which were conducted on various materials in order to study the variation of work function, $\phi_{2}$, of these materials with temperature and time. Four different types of collectors are under evaluation. These are:

(1) Impregnated nickel collectors.

(2) Oxide-coated nickel base collectors.

(3) Molybdenum collectors.

(4) Nickel collectors.

A low work function collector is one of the primary prerequisites in the development of an efficient thermionic power transducer. Before describing the work which has been done, the theory will be reviewed briefly.

The nomenclature is as follows:

Symbol

$\mathrm{A}_{1}$

$\mathrm{J}_{0}$

$\mathrm{J}_{\mathrm{R}}$

$\mathrm{k}$

$P_{\max }$

$q_{i}$

$\mathrm{T}_{1}$

$\mathrm{T}_{2}$

Vo
Description

Richardson's constant

Diode current density

Current density at critical point

Boltzmann's constant

Point of maximum power density

Heat loss

Emitter temperature

Collector temperature

Diode output voltage
Units

$120 \mathrm{amps} / \mathrm{cm}^{2}-{ }^{\circ} \mathrm{K}^{2}$

$\mathrm{amps} / \mathrm{cm}^{2}$

$\mathrm{amps} / \mathrm{cm}^{2}$

$8.61 \times 10^{-5}$ volts $/{ }^{\circ} \mathrm{K}$

watts $/ \mathrm{cm}$

watts

${ }^{\circ} \mathrm{K}$

${ }^{\circ} \mathrm{K}$

volts 
Symbol

$\mathrm{V}_{\text {OC }}$

$\mathrm{V}_{\mathrm{R}}$

$\mathrm{V}_{\mathrm{T}}$

W

$\phi_{2}$

o

$\eta$
Description

Open circuit voltage

Voltage at critical point

Voltage thermal equivalent

Diode spacing

Work function

Space charge barrier

Efficiency
Units

volts

volts

$\mathrm{T} / 11,600$

$\mathrm{cm}$

volts

volts

percent

The current flow per sq $\mathrm{cm}$ of emissive surface in a thermionic diode, assuming that the back emission is small, is equal to

$$
\mathrm{J}_{\mathrm{o}}=\mathrm{A}_{1} \mathrm{~T}_{1}^{2} \exp \left(-\phi_{2} / \mathrm{kT}_{1}\right) \exp \left(-\mathrm{V}_{\mathrm{o}} / \mathrm{k} \mathrm{T}_{1}\right) \exp \left(-\delta / \mathrm{kT}_{1}\right)
$$

and the power output,

$$
\mathrm{P}_{0}=\mathrm{J}_{0} \mathrm{~V}_{0}-\mathrm{P}_{1}
$$

and the efficiency,

$$
\eta=\frac{J_{0} V_{0}-P_{1}}{\Sigma q_{i}} .
$$

From equations (1), (2) and (3), we can see that a small decrease in $\phi_{2}$ results in a large increase in current densities and, therefore, higher power and efficiencies.

The experimental data was analyzed in the following way. For small current densities, it can be assumed that $\delta=0$ and, therefore, equation (1) becomes

$$
\mathrm{J}_{\mathrm{o}}=\mathrm{A}_{1} \mathrm{~T}_{1}^{2} \mathrm{e}^{-\phi_{2} / \mathrm{kT}} \mathrm{e}^{\mathrm{V}_{\mathrm{o}} / \mathrm{kT} \mathrm{T}_{1}}
$$


The experimental dependence of current density on output voltage plotted semilogarithmically gives a straight line. As the current densities increase, the space charge barrier should be considered. The effect of the space charge barrier on the current-voltage curve causes departure from straight line dependency. The "critical" point occurs at the point of tangency of the straight line portion with the curved portion.

From the straight line portion of the plot (see sketch below), we obtain the following information:

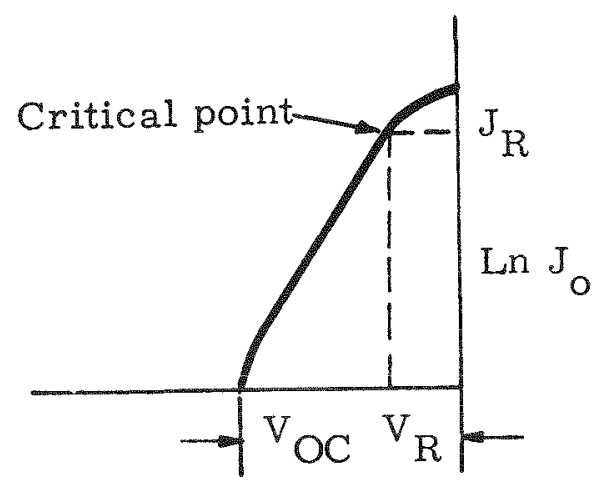

Taking the natural log of Eq. (3) gives

$$
\text { en } J_{0}=\ln \mathrm{AT}_{1}^{2}-\frac{\mathrm{V}_{0}}{\mathrm{kT}}-\frac{\phi_{2}}{\mathrm{kT}_{1}} \text {. }
$$

Assuming $\phi_{2}$ constant for a fixed $\mathrm{T}_{1}$ and taking the first derivative of $J_{0}$ with respect to $V_{0}$, we have

$$
\frac{\mathrm{d} \ell \mathrm{n} \mathrm{J}_{0}}{\mathrm{~d} \nabla_{\mathrm{O}}}=-\frac{1}{\mathrm{kT}}
$$

or

$$
\mathrm{T}_{1}=\frac{\Delta \mathrm{V}}{\mathrm{k} \Delta \ell \mathrm{n} \mathrm{J}_{0}}
$$

Therefore, the temperature of the emitter can be calculated from Eq. (6). 
Also, solving for $\phi_{2}$ from Eq. (3), we find that the work function of the collector is given by

$$
\phi_{2}=\mathrm{kT}_{1} \text { \&n } \frac{\mathrm{AT}_{1}^{2}}{\mathrm{~J}_{\mathrm{o}}}-\mathrm{V}_{\mathrm{o}}
$$

Other characteristics of a diode that can be found from a $J_{0}-V_{0}$ curve are the spacing between the emitter and collector, collector temperature, and maximum power output.

$$
\begin{aligned}
& \mathrm{W}=\frac{7.729 \times 10^{-12}}{\mathrm{~J}_{\mathrm{R}}} \mathrm{T}^{3 / 4} \\
& \mathrm{~V}_{\text {oc }}=\phi_{2} \frac{\mathrm{T}_{1}}{\mathrm{~T}_{2}}-1+2 \mathrm{kT}_{1} \text { ln } \frac{\mathrm{T}_{1}}{\mathrm{~T}_{2}} \\
& \mathrm{P}_{\max }=3.7 \times 10^{-6} \mathrm{~V}_{\mathrm{T}} 1 / 2 \frac{\mathrm{V}_{2}}{\mathrm{~W}}
\end{aligned}
$$

\section{Impregnated Nickel Collector}

Four identical nickel collector tubes with Type $S$ impregnant in a porous nickel base were designed. The emitters were ordered and received. The presses, getters, glass tubing, nickel sheet for the collectors and the thermocouples are presently in stock. Figure 13 is a drawing of the nickel collector tube. No tests of this design have been performed to date.

\section{Oxide-Coated Nickel Base Collectors}

Prior to this report period, progress in determining the true work function of oxide-coated nickel consisted of tests with assemblies which incorporated oxide-coated nickel as a collector, and a relatively low temperature $\left(1600^{\circ} \mathrm{K}\right)$ emitter such as Type B impregnated tungsten. However, the oxide-coated nickel collector work function value invariably was masked by the deposition of barium and other contaminating agents on the collector. If a pure metal such as tungsten or molybdenum were used as the emitter, the high temperature $\left(2500^{\circ} \mathrm{K}\right)$ necessary to produce suitable emission would, by conduction through the sapphire separators and by radiation, heat the oxide-coated collector to the point of decomposition. 


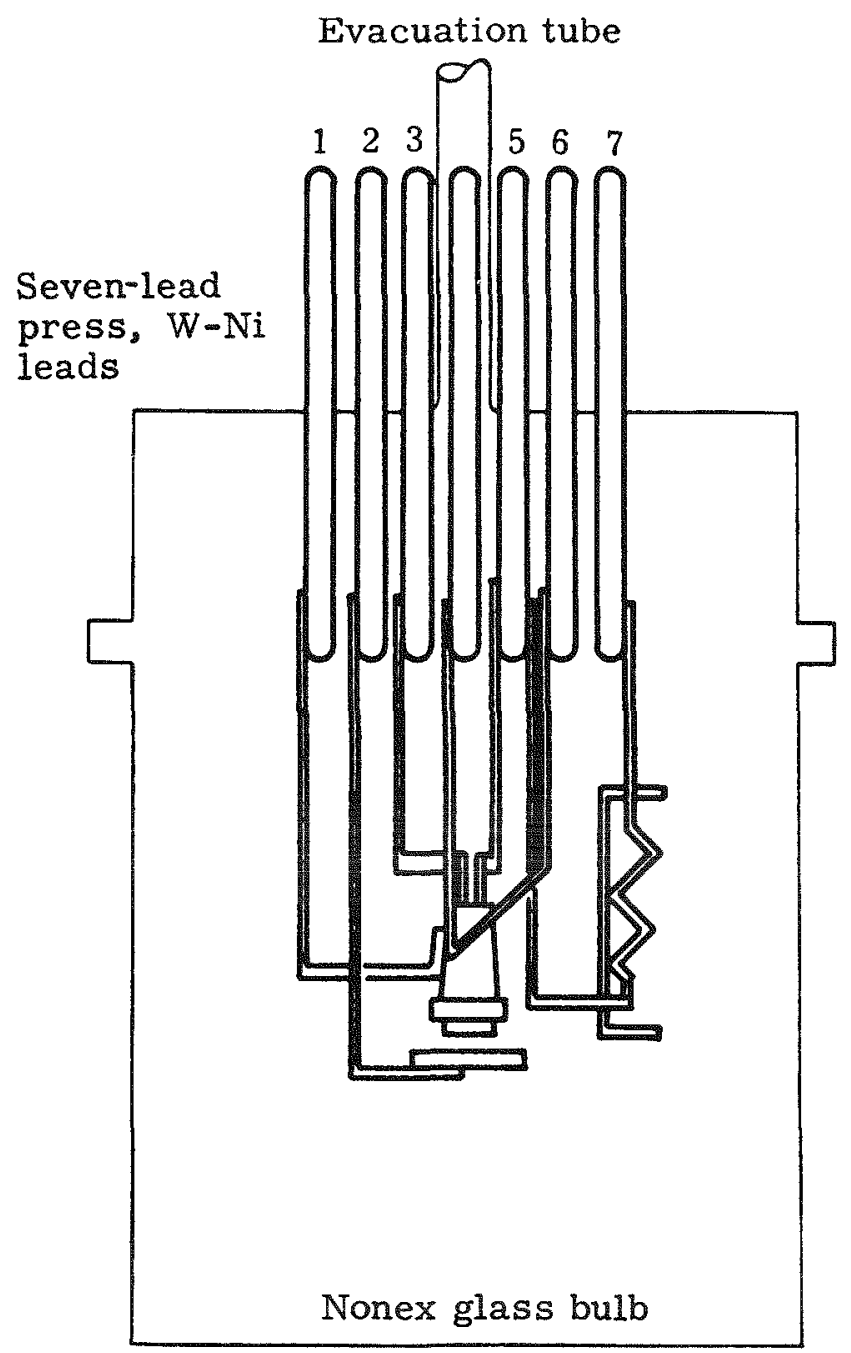

1. Emitter, with internal coiled- coil heater

2. Nickel collector

3. Heater

4. Thermocouple, Pt--Pt $10 \% \mathrm{Rh}$

5. Heater, getter (barium)

6. Thermocouple

7. Getter (barium)

Fig. 13. Nickel Collector Tube 
Therefore, to determine the work function of an oxide-coated, nickel base specimen, a diode was designed and constructed which incorporated an oxide-coated nickel emitter and a molybdenum collector to achieve electrical continuity for the assembly. The collector was designed to be heated and outgassed by an RF induction furnace (see Fig. 14).

\section{Molybdenum Collectors}

Tests were run using a Type B emitter and a pure molybdenum collector, with an emitter temperature of $1540^{\circ} \mathrm{K}$. During the first 20 minutes, the rate of change of $\phi_{2}$ was quite large due to deposition of barium. This can be seen in Fig. 15, Collector Work Function vs. Time. After approximately an hour, a minumum value of $\phi_{2}$ was obtained at 1.9 volts. However, as more time elapsed, $\phi_{2}$ slowly increased to a value of about 2.2 volts. This indicates that an excessive amount of barium must have been deposited on the collector.

Another test run was made with the emitter operating at a temperature lower than $1500^{\circ} \mathrm{K}$ in an effort to decrease the rate of change of $\phi_{2}$ by reducing the barium deposition on the collector. After 26 hours, the work function was observed to be still decreasing towards 2.0 volts (see Fig. 16).

An alternate method for reducing $\phi_{2}$ was tried in the next run. The deposition of barium on the collector was expedited until a minimum value of $\phi_{2}$ was. obtained. The temperature of the cathode was then lowered to curtail further deposition of barium and thus to hold $\phi_{2}$ at this minimum. The high emitter temperature in this run was $1500^{\circ} \mathrm{K}$. After the minimum of 1.8 volts was reached, the emitter temperature was lowered by $125^{\circ} \mathrm{K}$ (see Fig. 17). However, $\phi_{2}$ increased very sharply up to 2.3 volts instead of holding at 1.8 volts. This shows that the temperature decrease must be very fast, almost instantaneous, in order to stop further deposition.

Results obtained for the Type B emitter and Type B collector are given in Table 3 . These results show $\phi_{2}$ to be about 2.5 volts, which is rather high. A possible explanation is that the collector was too hot. It was observed to be glowing. These tests were suspended since molybdenum collectors not only give lower work functions than the Type B collector, but they can also be machined more easily. 


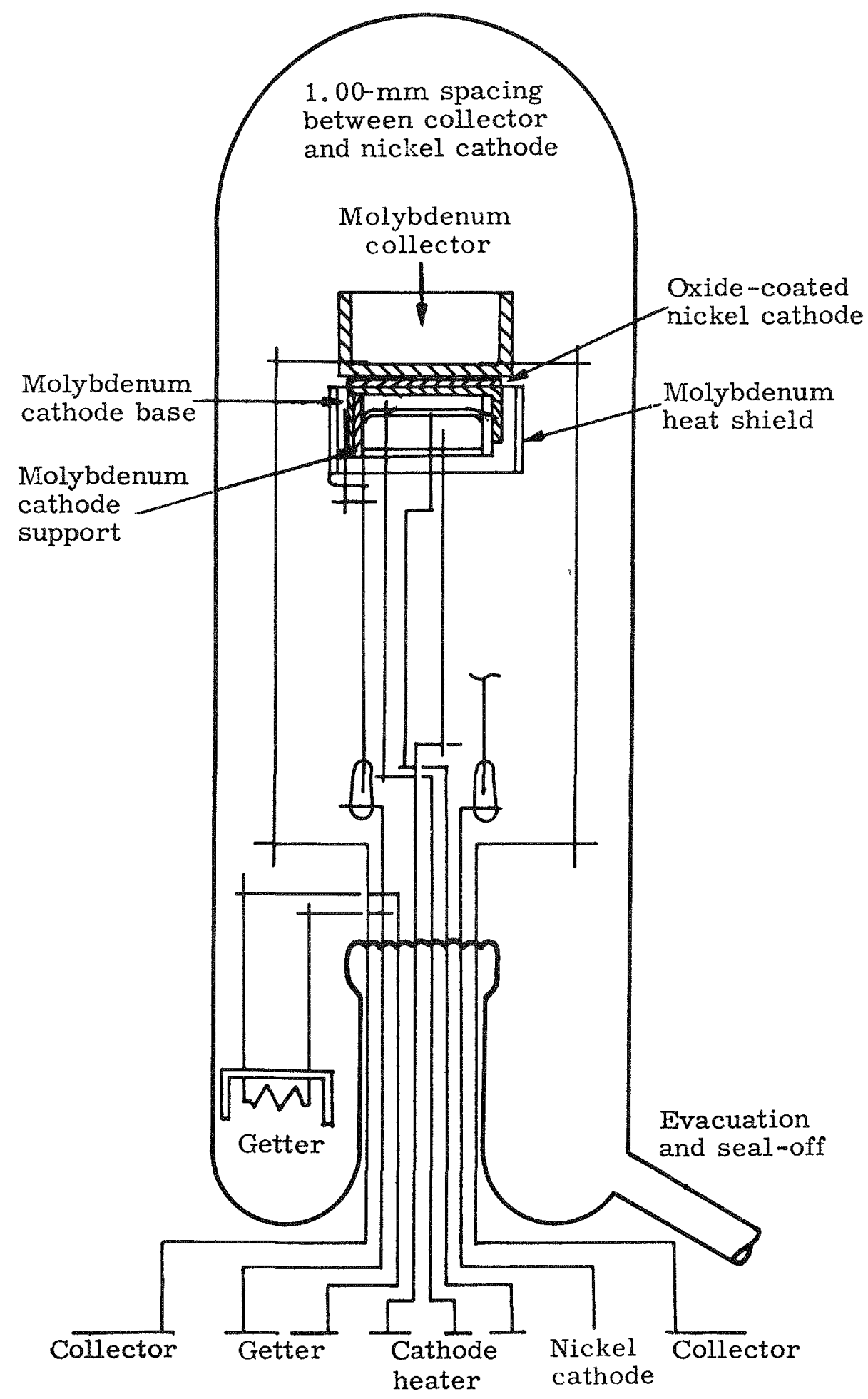

Fig. 14. Oxide-Coated, Nickel-Base Cathode Tube 


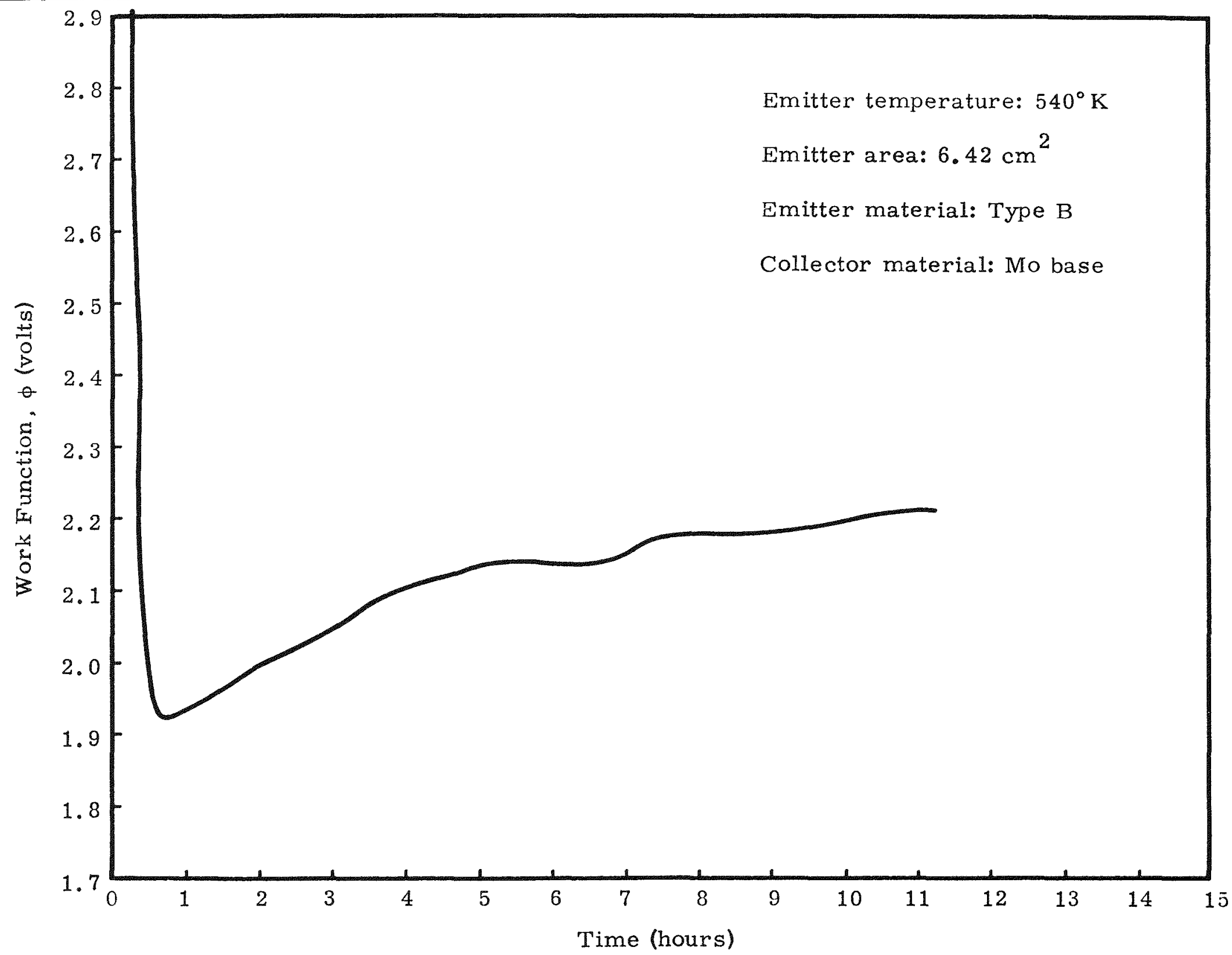

Fig. 15. Collector Work Function vs Time 


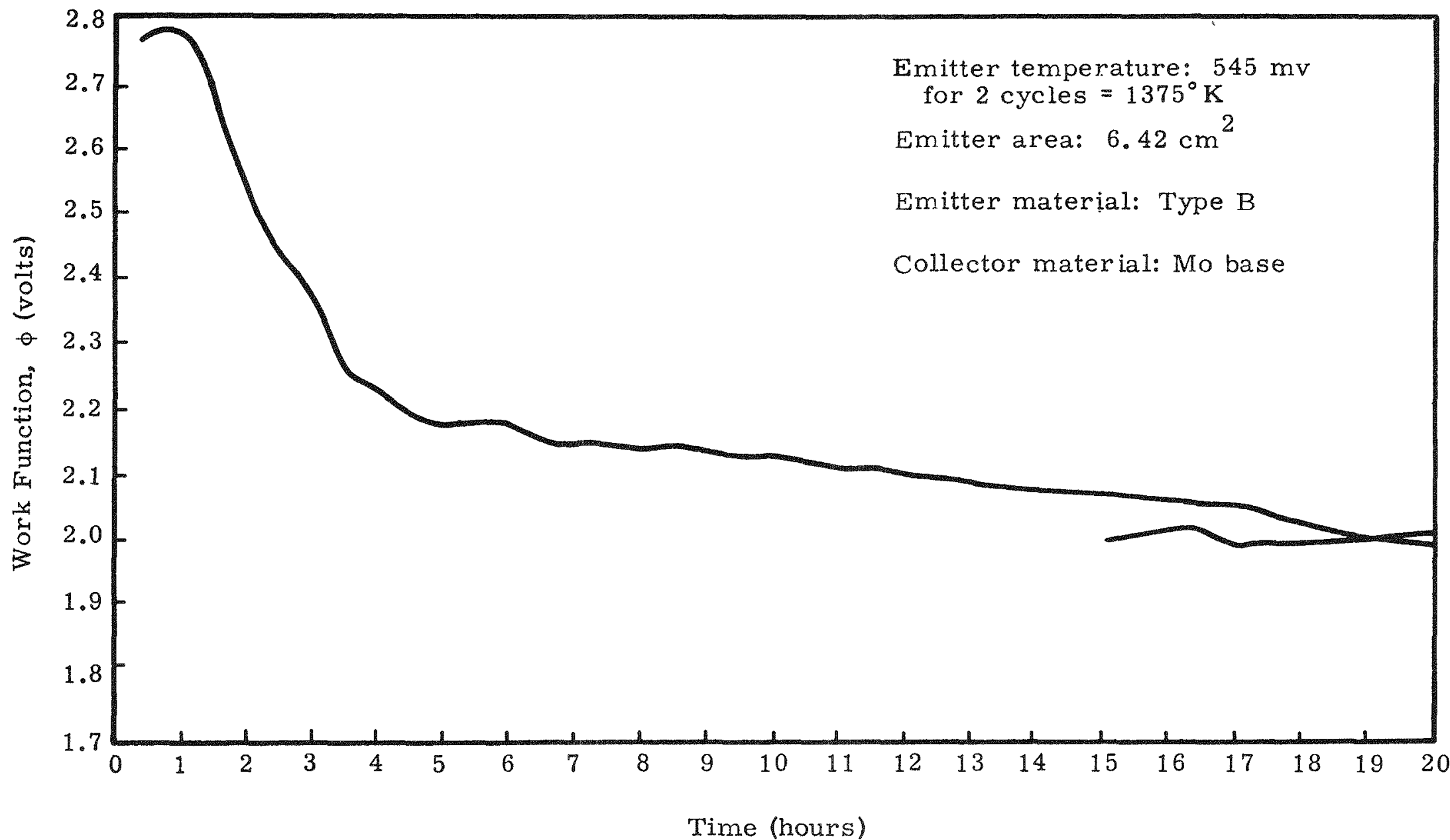

Fig. 16. Collector Work Function vs Time 


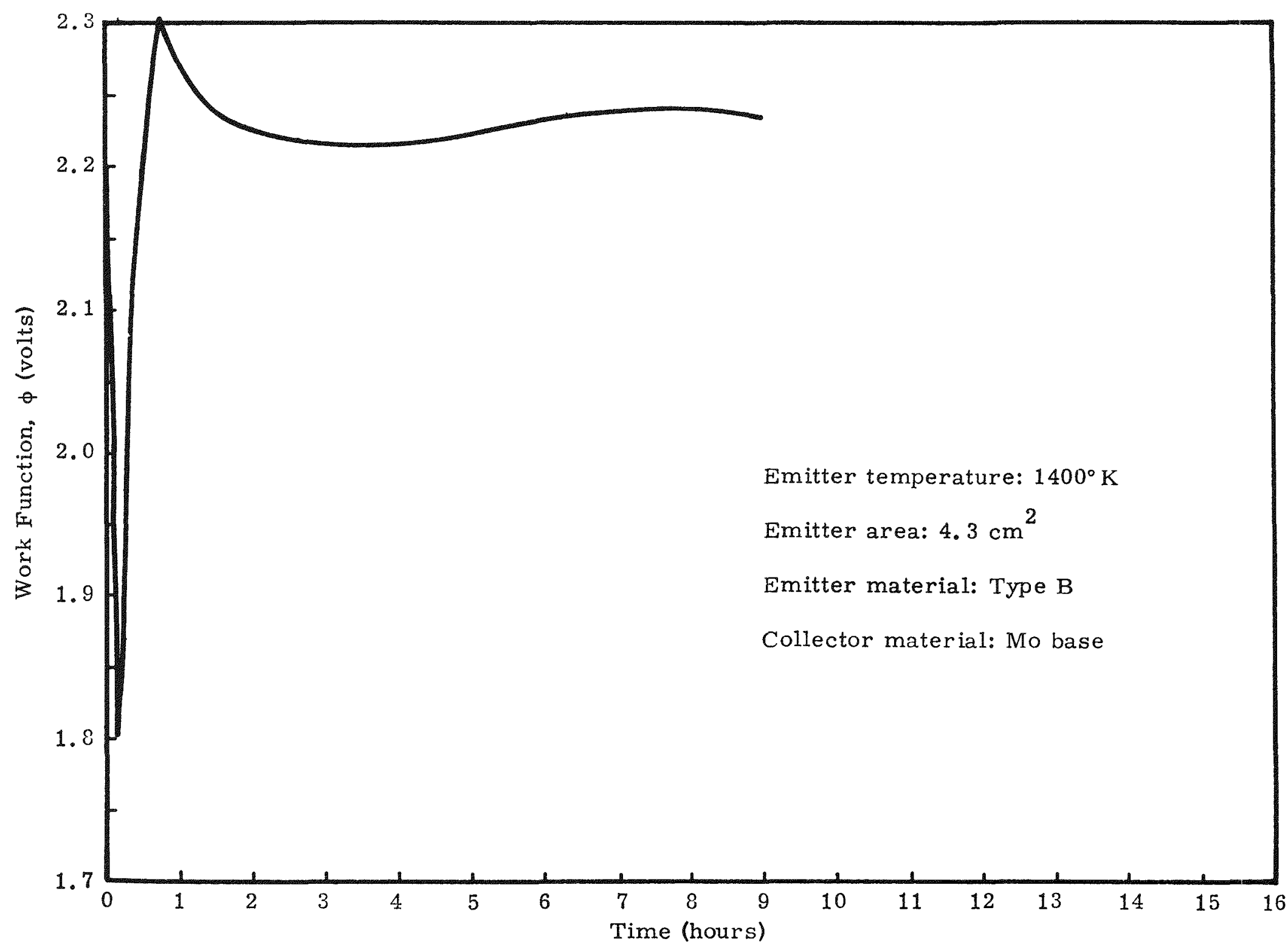

Fig. 17. Collector Work Function vs Time 
TABLE 3

Type B Collector--Type B Emitter

$\underset{\left({ }^{\circ} \mathrm{K}\right)}{\operatorname{Tmitter}}$

1460

1460

1540 $\phi$ Collector (volts)

2.44

2.44

2.74

The next series of tests was made to study the work function characteristics of a nickel-based oxide-coated cathode. The cathode was placed opposite a plain molybdenum plate. Emission currents from the cathode were measured at various temperatures. From these, the work function of the cathode at each temperature was calculated. These values are shown in the first part of Table 4 . The average value of $\phi_{2}$ is 2.4 volts.

The collector plate was then changed from molybdenum to titanium and emission tests were run again. The value of the work function of the nickel cathode was then observed to be around 2.0 volts. These values are shown in the last part of Table 4. Assuming that all other parameters are the same, the decrease in work function of the nickel emitter in the presence of titanium can be ascribed to the fact that titanium is a better getter than molybdenum, so there was better vacuum near the nickel cathode with titanium as a collector.

Even though low work functions were obtained, further tests were necessary in order to establish a definite procedure for stabilizing these low values. It was planned to carry out these experiments with a Type $B$ emitter and a molybdenum collector sealed in an evacuated tube. This would also facilitate testing for longer periods. 
TABLE 4

Oxide-Coated Cathodes

\begin{tabular}{|c|c|c|}
\hline & & Short Circuit \\
\hline $\begin{array}{c}\mathrm{T} \\
\left({ }^{\circ} \mathrm{C}\right)\end{array}$ & $\stackrel{\phi}{(V)}$ & $\begin{array}{l}\text { Current } \\
\text { (ma) }\end{array}$ \\
\hline
\end{tabular}

Cataphoritic coating (Raytheon) moly collector

Cataphoritic coating (Raytheon) moly collector

Cataphoritic coating (Raytheon) moly collector

Above cathode heated in hydrogen-argon atmosphere to $882^{\circ} \mathrm{C}$

Cataphoritic coating (Raytheon) titanium collector

Cataphoritic coating (Raytheon) titanium collector

Cataphoritic coating (Raytheon) titanium collector

Spraying coating (MIT)

titanium collector

Spraying coating (MIT)

titanium collector
788

2.34

0.4

860

2.5

0.3
2.55

$860 \quad 2.51$

966

2.4

945

2.45

930

2.0

889

2.1

878

2.0

In order to determine the work function of the molybdenum, a Type $B$ impregnated tungsten emitter was incorporated in a tube with no molybdenum collector as shown in Fig. 18. Sapphire spheres control the interelectrode spacing so that, at room temperature, the spacing was 0.0006 inch. It is expected however, that, at high temperatures, this separation will increase due to thermal expansion of the sapphire spheres.

A feature of the diode is the collector design. The configuration makes the molybdenum collector readily accessible for $\mathrm{RF}$ induction heating during initial diode outgassing and activation. The collector is of sufficient length so that good coupling with the RF unit can be achieved. 


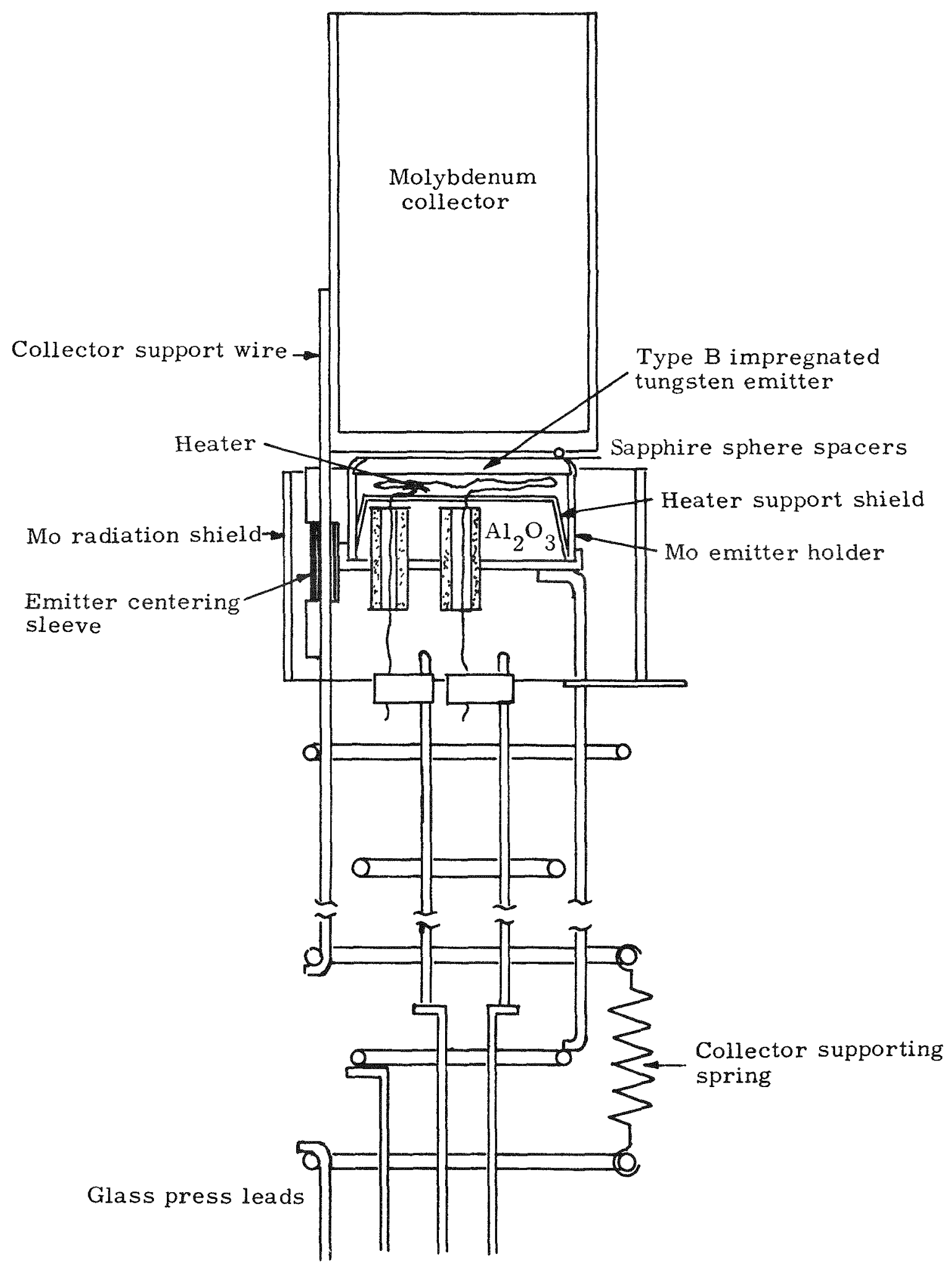

Fig. 18. Type B Impregnated Tungsten Emitter Assembly 
This length also minimizes the possibility of damaging the emitter structure and the leads through the glass press by heating caused by inductive coupling. The long cylindrical walls of the collector permit cooling by radiation while operating and testing the emitter at high temperatures.

The purpose of these tests was to determine the effect of barium emitted from the Type B emitter on the work function of the collector as a function of operating time.

The diode consists of the following main parts:

(1) A Type B emitter encased in a molybdenum sleeve (see Fig. 19).

(2) Coil heaters in the hollow molybdenum sleeve to heat the emitter.

(3) A hollow molybdenum cylinder with a flat bottom used as the collector, and which is spaced 0.001 inch from the emitter by sapphire spheres.

The tube was assembled with great care in keeping all parts as clean as possible and allowing a minimum exposure of the emitter to the atmos phere. The collector and radiation shield were fired in a vacuum at $2000^{\circ} \mathrm{F}$ for 10 minutes before assembly.

After the tube was completely assembled, it was connected to a glass vacuum system. A vacuum of $10^{-7} \mathrm{~mm}$ of $\mathrm{Hg}$ was obtained. The glass tube was outgassed by means of an oven provided in the vacuum system. It was baked out at a temperature of $700^{\circ} \mathrm{F}$ for 24 hours.

The entire glass system, including the glass ways and cold trap, was outgassed to obtain the best vacuum possible. The emitter, collector and radiation shield were outgassed by the emitter coil heater and induction heating. The outgassing rate was adjusted so that the pressure never rose above $10^{-5} \mathrm{~mm}$ of $\mathrm{Hg}$. Once the temperature of the emitter and collector reached $1500^{\circ} \mathrm{K}$, this temperature was maintained for $1-1 / 2$ hours. At theend of the 1-1/2 hours of outgassing, the induction heater was maintained at $1500^{\circ} \mathrm{K}$.

The output voltage at a fixed output current of 40 ma was recorded as a function of time. The emitter temperature, vacuum condition, and voltage across the emitter heater were continuously monitored. Sufficient data were taken to determine the work function over a 122-hour period. After 12 hours of operation, the work function was essentially constant with time, as can be seen in Fig. 20. The stabilized value of 


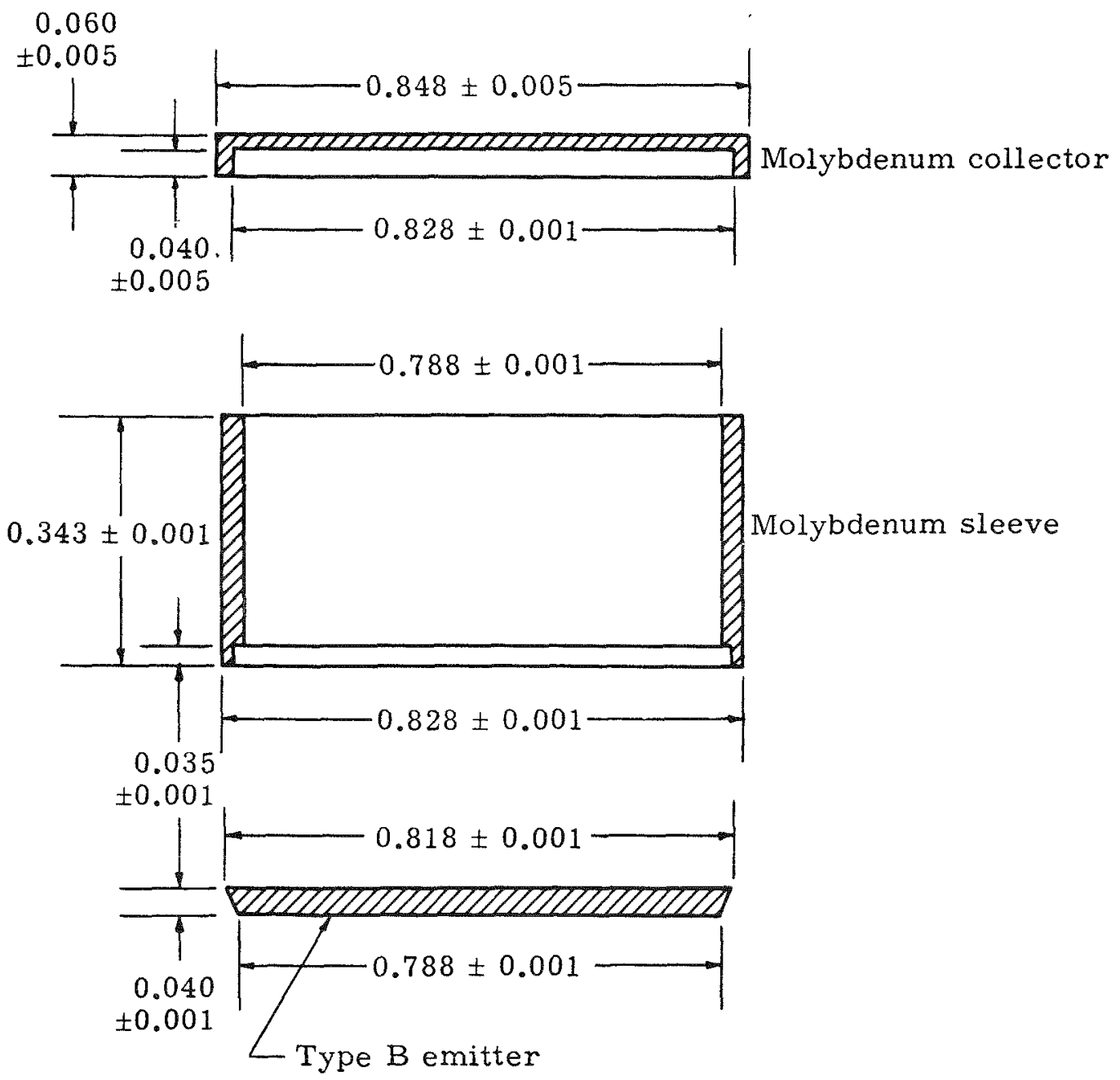

All dimensions given in inches.

Fig. 19. Type B Emitter and Molybdenum Sleeve Assembly 


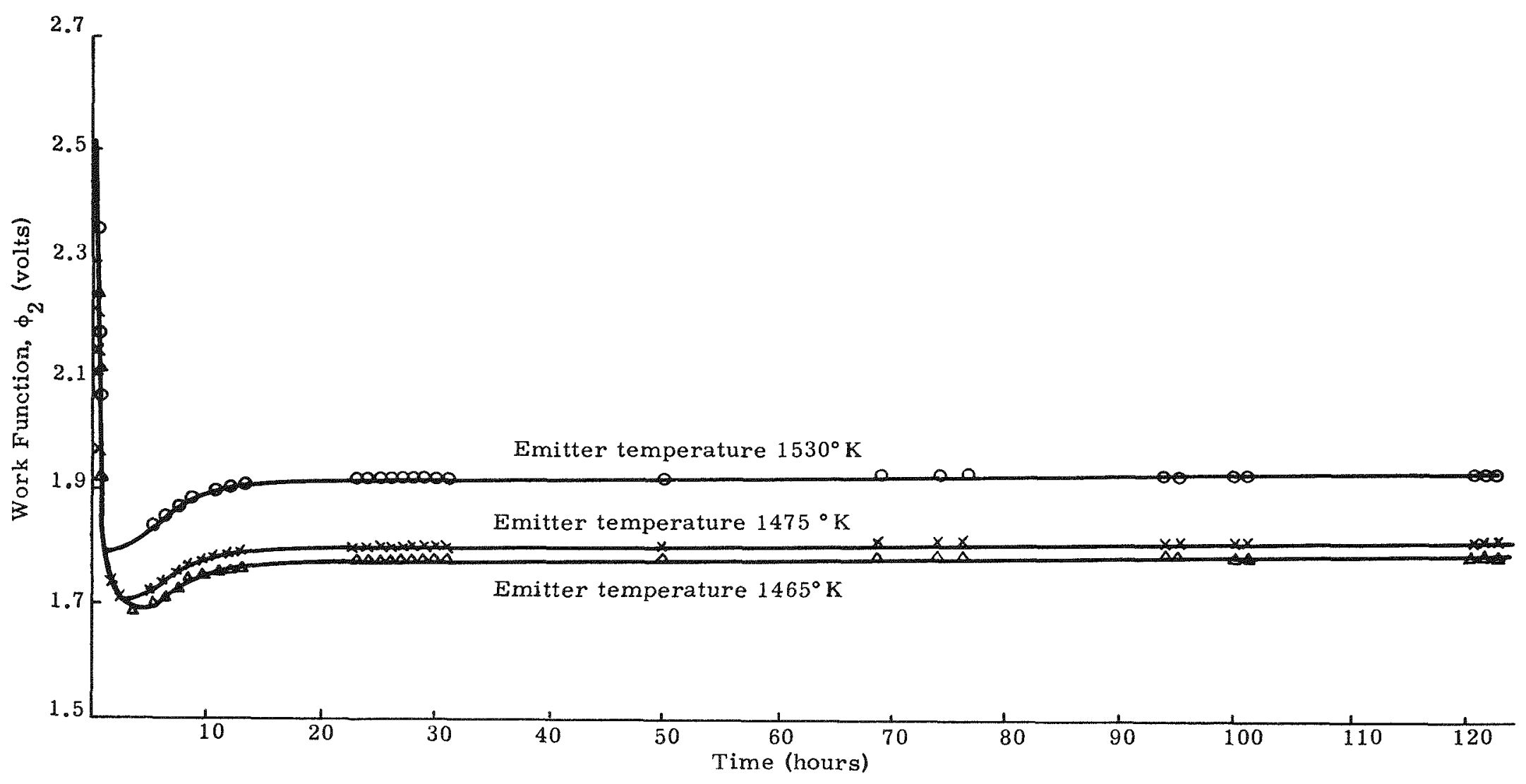

Fig. 20. Collector Work Function vs Time 
the work function is either $1.95,1.82$ or 1.80 volts, depending on which value is used for the temperature of the emitter. Pyrometer temperature measurements, with correction for spectral emissivity, indicate a temperature of $1530^{\circ} \mathrm{K}$, resulting in a work function of 1.95 volts. Pyrometer measurements applying the manufacturer's correction indicate a temperature of $1475^{\circ} \mathrm{K}$, corresponding to a work function of 1.82 volts. The emitter temperature obtained from the $\mathrm{I}-\mathrm{V}$ curves is $1465^{\circ} \mathrm{K}$, which corresponds to a work function of 1.80 volts. The I-V curves were taken at large time intervals, and the temperatures obtained are in good agreement with each other.

The important result is that the primary objective of this test series was accomplished, in that a low work function on a molybdenum collector was obtained and maintained for an indefinite period. A work function of approximately 1.82 to 1.85 volts was obtained and, after 12 hours of operation, it remained constant at this value for 110 hours. The test was terminated because the heater was beginning to weaken. In order to prove reproducibility of the work function after the tube was inoperative for some time, the tube was left in a cold condition for 5 days and then tested again. The work function had not changed, and all points were constant over a 2-hour period.

Considerations as to why and how such a stability occured point up several interesting factors. First, the emitter was outgassed at such a rate and period of time that the greatest part of barium emission from a Type $B$ cathode occured during outgassing while the collector was hot. Therefore, little or no barium was deposited on the collector. When the collector was cooled down, the rate of barium emission from the emitter was only sufficient to deposit a partial coverage on the collector, resulting in a low work function. The barium emission was not sufficient to deposit a thick layer, which would have resulted in a work function on the collector equal to that of barium, 2.2 volts. Secondly, the environment inside the vacuum tube was of constant quality. The vacuum, although it was not measured, probably was better than $10^{-6} \mathrm{~mm} \mathrm{Hg}$ (Before sealoff, the vacuum was $10^{-5} \mathrm{~mm} \mathrm{Hg}$ without having activated the getter.) During the construction of the tube, great care was taken to keep all parts as clean as possible, thus minimizing any poisoning effects of the emitter or collector by impurities. Also, the slow outgassing evaporated agents that might otherwise have poisoned the cathodes before the electrodes were very hot ${ }_{2}$ at which time they are more susceptible to oxidation.

The work function of molybdenum was originally about 3 volts and started decreasing to a value of 1.70 volts within a matter of minutes. 
Then, it slowly rose to the constant values as reported, depending on what temperature was used for the calculation of $\phi_{2}{ }^{\circ}$ The time it took $\phi_{2}$ to reach a stable value was less than 12 hours. Although the change from the minimum to the steady $\phi$ was relatively small (0.10 volt), it is obvious that excessive barium was deposited on the collector, thus altering its work function. It is hoped that this can be eliminated by longer outgassing of the emitter while the collector is kept at $1500^{\circ} \mathrm{K}$ and a constant value of 1.7 volts achieved.

Although this tube was not designed for high power density, it produced a power output of 0.135 watt per square inch which is an excellent value for the spacing of the device. The maximum power output throughout the 122 hours, plus the retesting 5 days later, was constant within $5 \%$ of experimental error.

The spacing obtained from the $1-\mathrm{V}$ data is relatively large. This is because the emitter surface is off flatness by 0.0004 to 0.0006 inch. and the spheres used to separate the cathodes were protruding by 0.0003 to 0.0005 inch. When the thermal expansion of the shperes is included, a spacing of 0.0015 inch $(0.0038 \mathrm{~cm})$ is fairly realistic.

\section{Nickel Collectors}

During this period, parts were completed for the nickel collectorType B impregnated tungsten emitter diode. When assembled, this tube was identical to the configuration described for the molybdenum collector except for the collector materials. The activation and testing will follow the procedures mentioned previously.

\section{Study of Vacuum Improvement in a Bell Jar System}

The development of an assembly designed to provide a vacuum better than $10^{-6} \mathrm{~mm} \mathrm{Hg}$ was initiated in November. Most of the parts have been machined and assembled. Figure 21 is a drawing of the setup.

The unit consists of a titanium housing within a glass bell jar. Initially, the metal housing is raised from its mating base plate. The glass bell jar is then evacuated to minimum pressure attainable with the system, generally about $10^{-6} \mathrm{~mm} \mathrm{Hg}$. The titanium housing is then mated with its base plate, forming an independent system in a vacuum equal to that of the glass bell jar system. The barium getter inside the titanium housing is then fired, and this should improve the smaller system's vacuum appreciably. 


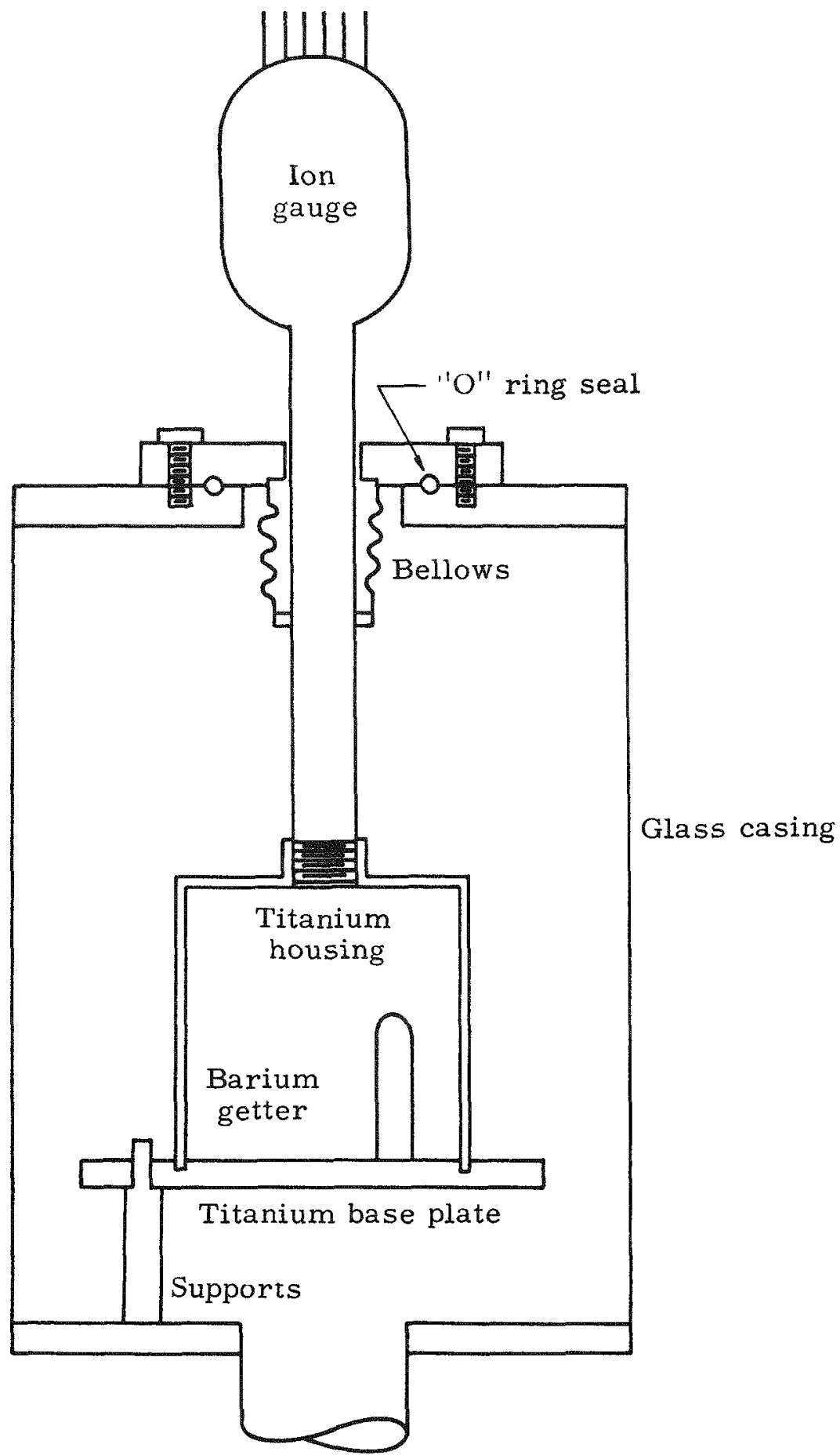

Evacuation Outlet

Fig. 21. Apparatus for Vacuum Improvement In a Bell Jar System 


\section{SUBTASK 5.3--DEVELOPMENT OF LOW POWER THERMIONIC GENERATOR*}

The objective of this subtask is to develop, design, fabricate, and test an operational power system coupling a thermionic conversion device to a radioisotope heat source. The thermionic development is being performed by Thermo Electron Engineering Corporation of Cambridge, Massachusetts, as a subcontractor to The Martin Company. The work on the isotopic heat source and all other related work is being done by Martin Nuclear Division.

At the beginning of the quarter, plans to fuel a 2 - to 3 - watt thermionic prototype with a Cerium-144 isotopic heat source during the first week in December were still in effect. As work progressed, it became apparent that there was little to be gained by proceeding with these plans. Much more could be learned about both the heat source and the thermionic device by working with each component individually. The electrically heated thermionic units are more flexible than a ceriumfueled unit, and therefore, it is possible to obtain much more basic data from them. This also applies to the heat source. Much more basic data on the Cerium-144 pellets and their interaction with the molybdenum fuel capsule are needed before heat sources can be designed and fabricated with a high degree of reliability. Therefore, at presentations given in Germantown on November 13, 1959, and at the New York Operations Office on November 23,1959, it was recommended that the fueling of the thermionic unit be indefinitely postponed, but that the molybdenum fuel capsule be fueled as planned.

The plans which had been made and the work performed in preparation for the fueling were extremely helpful. This work highlighted all of the problems one can expect when attempting to fuel a thermionic unit with a radioactive heat source such as Cerium-144. The importance of not contaminating the electrode surfaces of the thermionic unit during fueling became apparent. Also, the difficulties involved in assembling a thermionic unit by remote operation in a hot cell are better appreciated now. Thus, the work which was done was quite valuable in developing the art of fueling thermionic generators.

\section{A. THERMOELECTRON ENGINEERING CORPORATION (TEECO) EFFORTS}

The work performed by TEECO can be conveniently broken down for reporting purposes as follows: 
(1) Prototype Development。

(2) Heat Transfer Study and Tests.

(3) Vacuum Tests in Prototype Shells。

(4) Cathode and Sapphire Support Creep Tests.

(5) Parametric Study.

\section{Prototype Development}

Work in this quarter was devoted to prototypes PT3, PT4, PT6, and Unit 1A。Prototypes PT3, PT4, and PT6 were all designed as versions of the two-stage thermionic unit which was to be fueled with the Cerium-144 isotopic heat source. Figure 22 is assembly drawing of PT6. Unit 1A was developed after the decision had been made not to fuel a thermionic unit with a radioisotope at this time. Unit $1 \mathrm{~A}$ is a single-stage unit, the purpose of which is to prove the feasibility and compatibility of the design features, each of which was developed more or less individually. The objectives of the development effort on this unit were as follows: to get the unit operating by reproducible methods; to be able to accurately predict the performance of the generator; and to be able to maintain this performance for an extended period of time. It was not an objective to try to achieve a highly efficient generator. This would be accomplished by an orderly improvement of the $1 \mathrm{~A}$ unit.

Unit PT3. All the necessary parts for this two-stage prototype were ready and as sembly was under way, as scheduled, at the beginning of October.

The prototype housing was made of monel and consisted of two identical halves. Monel was chosen because it can be heliarc-welded with the same welding equipment required for the molybddenum fuel capsule. After the final machining, thhe housing was tested for leaks by the differential absorption method. This test showed that the housing did leak. Investigation into the reason for this leak revealed that only forged monel is nonporous enough to be leakproof. Forged monel was not readily available at this time, so it was decided to use titanium for the housing of the unit.

Unit PT4。This unit was essentially the same as PT3. The only difference was in the choice of metals for the housing which, in this case, was titanium. The two halves of the housing were brazed together by a silver-copper eutectic which melts at $780^{\circ} \mathrm{C}$. The housings were leak checked before and after the final machining and were found to be leakproof. 


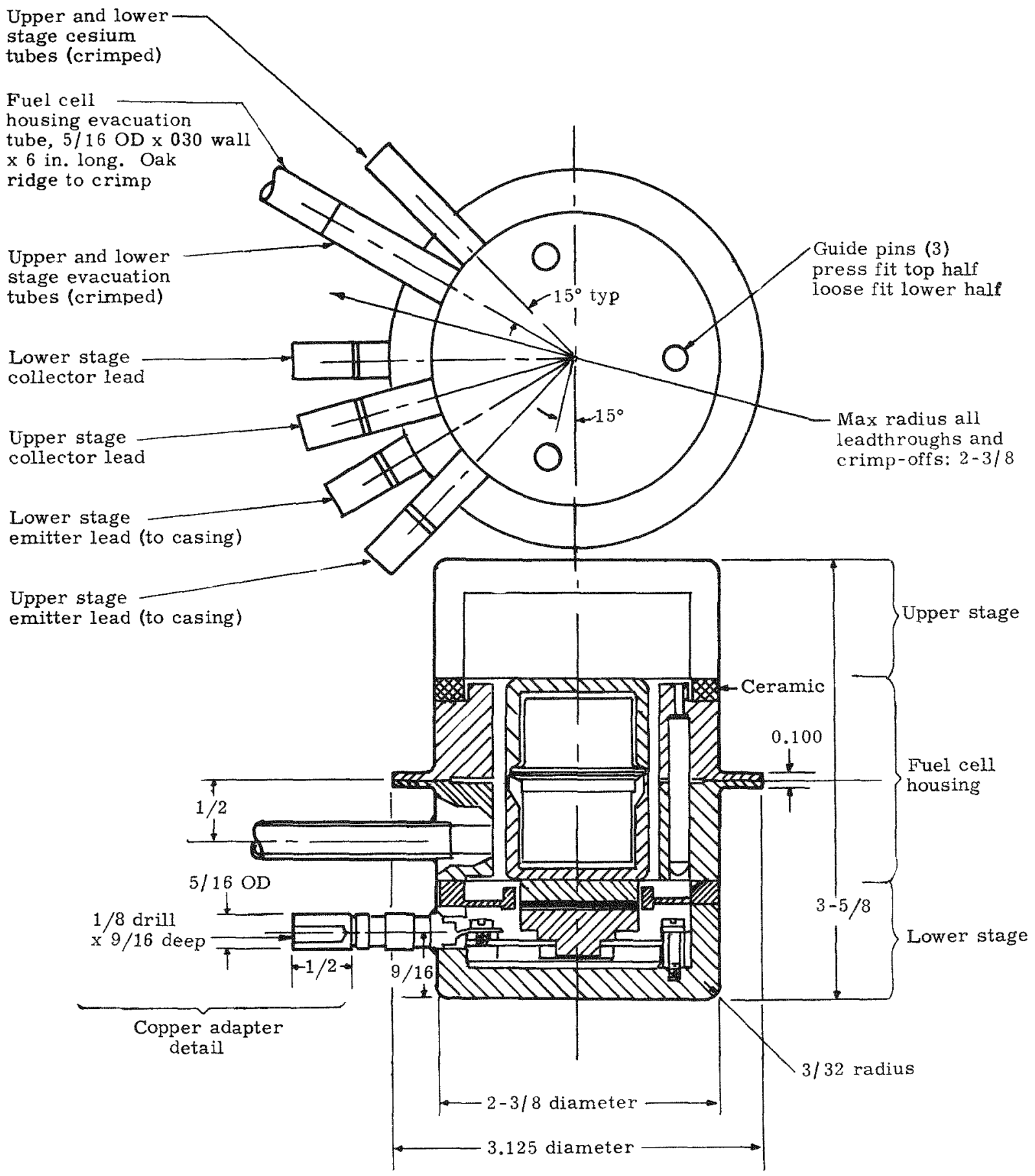

Fig. 22. Final Assembly PT6 
The unit was assembled with the electrical leadthrooughs brazed in place and the unit was evacuated with a twin vacuum system. The whole unit was placed in a chamber which was then evacuated. Provision for heating the housing was provided by an RF coil placed around the outer chamber, and the emitter of the unit was heated by an electrical heater.

The RF unit and the electrical heater were turned on. The temperature of the casing was kept below the melting point of the silver-copper eutectic braze. The unit was allowed to outgas for three hours; the length of the outgassing period was determined by the final stabilizing of inside and outside pressures considered suitable for the performance of the prototype. These conditions of pressure and temperature were maintained for another hour; then the temperature was raised until the braze melted and the unit was sealed. The temperature of the casing was kept $100^{\circ} \mathrm{C}$ below the brazing temperature for another three hours for further outgassing; then both the RF and electric heater powers were gradually decreased until the unit was cool.

The unit was next leak tested by filling the outer chamber with helium. An immediate increase in pressure indicated a leak in the unit.

The unit was removed from the evacuation system and subjected to a thorough leak test on the differential absorption leak detector. These tests showed that one of the leadthroughs was responsible for the leak. A crack had developed in the ceramic part of the leadthrough, causing the leak. This leadthrough was replaced by another, but the leak persisted. Thermal cycling tests on other specimens of the same type showed that the leadthroughs were unsuitable for use under the operating conditions of this unit.

The unit was machined open. An examination of the interior revealed the following:

(1) The leadthroughs developed leaks due to the cracking of a nickel part which had formed a brittle alloy with the silver-copper braze.

(2) The leaks in the leadthrough were also caused by cracks which might have developed due to thermal shock received during the heating, brazing and cooling cycle.

(3) One of the leaking leadthroughs, upon further examination, showed that the center tube was not adequately brazed to the ceramic.

(4) The molybdenum spring structure of the collector holder was permanently deformed due to annealing while brazing, thus reducing the effectiveness of the spring. This resulted in large emitter-collector spacing and improper positioning of the emitter structure. 
(5) One of the ceramic bushings, which insulated the collector holder from the housing, was broken. In addition, one of the screws supporting the collector holder was broken.

(6) By operating the heater with the housing removed, it was noted that the heat transfer between the emitter holder and the emitter was very poor. The temperature difference between the holder and the emitter was approximately $400^{\circ} \mathrm{F}$.

(7) During the time the unit maintained a good vacuum in the twin vacuum system, the two emitters were activated and tested for emission. A small amount of emission was observed for several minutes under a vacuum of $10^{-6} \mathrm{~mm} \mathrm{Hg}$ before the leads had broken.

Unit PT6. To avoid the objectionable features mentioned, prototype PT 4 was redesigned. This new prototype known as PT6, was a twostage unit with provision for an isotopic heat source. During assembly of this unit, it was noted that the delivery date for isotope fuel would not be realized, and the fueling of this two-stage prototype was indefinitely postponed. Instead, it was decided to concentrate efforts on an electrically heated prototype which would be a two-stage unit to be fabricated, tested and delivered to The Martin Company.

New leadthroughs were designed and manufactured. The modified design utilizes the principle of matched thermal expansion. These leadthroughs have kovar sleeves which are brazed to alumina ceramic by the powder metallurgy technique. The brazing material is copper. These leadthroughs successfully survived repeated thermal cycling between ambient and brazing temperatures.

Results of the tests carried out on prototype PT4 indicated that the molybdenum spring would not maintain the desired interelectrode spacing. The modified design uses a 0.001 -inch thick platinum-10\% rhodium foil which is brazed to the casing. The foil has atmospheric pressure acting on the exterior and a pressure of $10^{-6} \mathrm{~mm} \mathrm{Hg}$ inside. This pressure differential forces the collector against the emitter assembly. The foil was tested for leaks at ambient and operating temperatures.

The assembly for tests at ambient temperature consisted of a titanium container sealed off by a 0.002 -inch thick platinum-10\% rhodium foil. The whole assembly was placed on a differential sorption leak detector. The detector showed no leaks, which meant that leaks through the foil, if any, were not more than $10^{-8} \mathrm{cc} / \mathrm{sec}$. 
The arrangement for tests at operating temperatures consisted of a diode which was placed in a housing sealed off by a 0.002 -inch platinum$10 \%$ rhodium foil which also supported the emitter. A heater for the emitter was located inside the unit. The complete unit was heated by an external source and was contained in an inert atmosphere. The vacuum obtained in the unit was satisfactory and was held at elevated temperatures by the platinum-10\% rhodium foil.

The incomplete unit, PT6, was an ideal vehicle for leak testing the 1 mil platinum-10\% rhodium membrane. The tests showed that the membrane did have leaks. Photographic testing of all the platinum-10\% rhodium foil in stock indicated the presence of minute holes.

Unit 1A. Prototypes of the 1 series $\left(1 \mathrm{~A}, 1 \mathrm{~B}\right.$, etc $\left.{ }_{\circ}\right)$ will be designed for the power density of Cerium-144since many parts have been fabricated on this basis. These units will serve only as test generators because it is not planned to actually fuel a low powered thermionic unit with Cerium-144. Prototypes of the 2 series (2A, 2B, etc.,) will be based on the power density of Curium-242 and, therefore, will be much more efficient, smaller and lighter.

Fabrication of parts and the assembly of the prototype $1 \mathrm{~A}$ began in mid-November. Platinum-10\% rhodium foil 0.001 inch thick was used, despite the fact that it was not completely vacuum-tight. The reason for this choice over 0.002 -inch foil was to avoid doubling the heat losses with the thicker foil. The collector now has a rigid support. Molybdenum powder is included between the emitter and the holder to decrease the temperature film drop at this interface. (The use of molybdenum is explained in Section A.2.)

The assembly was placed in a bell jar without the housing, and the emitter was raised to the operating temperature. Output current vs voltage for this unit is shown in Fig. 23. After satisfactory operation, the unit was assembled in the casing, electrical leads were fastened to the leadthroughs and appropriate braze shims were provided. Figure 24 is a drawing of the assembly.

The unit was heated externally by an RF coil and internally by an electric heater to outgas the unit thoroughly before sealoff. Figure 25 is a diagram of the setup. The unit remained in this final stage for 3 hours, heated to $700^{\circ} \mathrm{C}$ as measured by an optical pyrometer. When a pressure of $5 \times 10^{-6} \mathrm{~mm} \mathrm{Hg}$ was maintained for one hour, the outgassing was considered sufficient. The temperature was then raised gradually, to avoid thermal shock on the ceramic part of the leadthroughs, to $780-800^{\circ} \mathrm{C}$, which is the melting point of the Ag-Cu eutectic. All of the final brazes were successful, but the cap did not seat correct- 


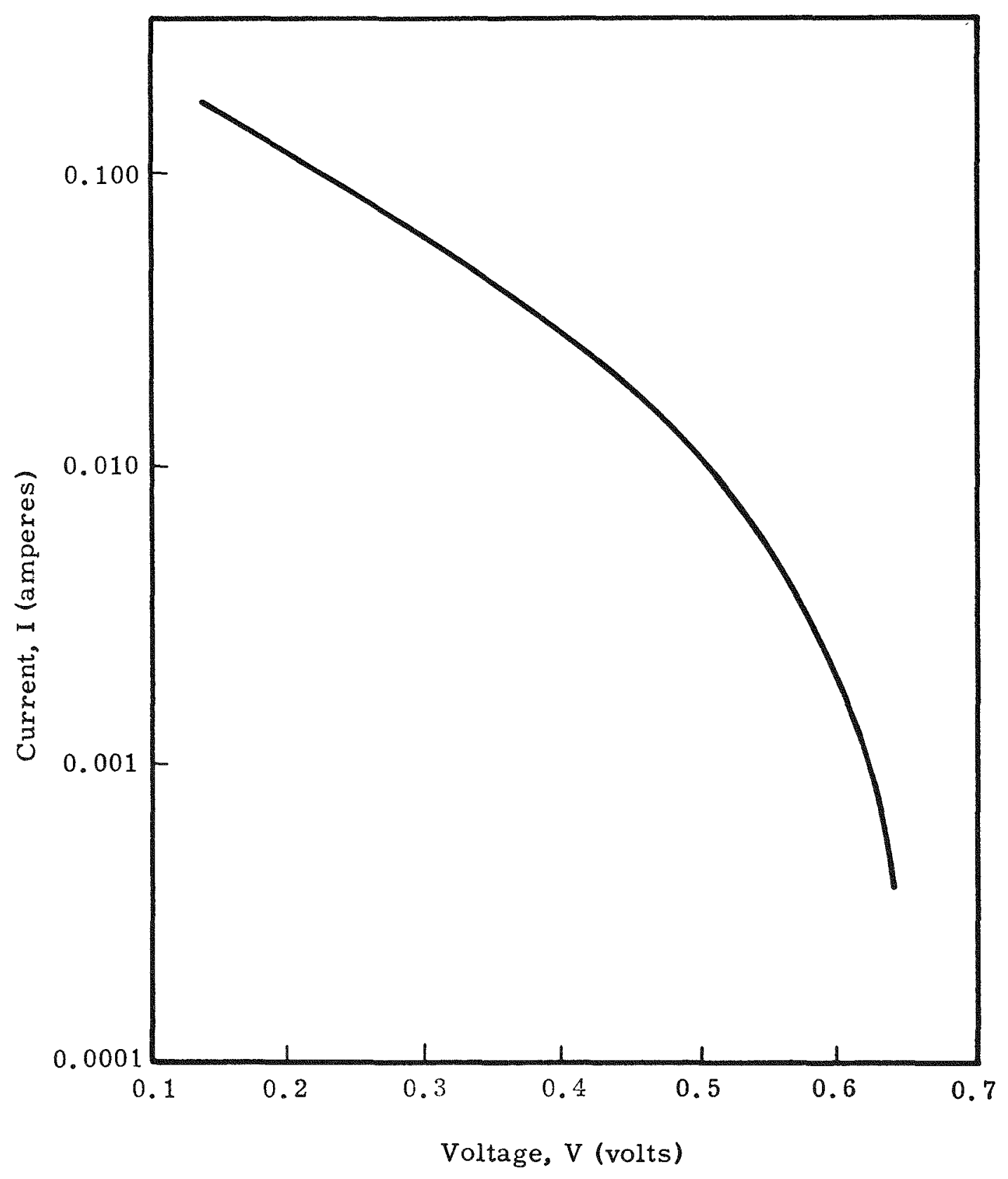

Fig. 23. Output Current, I, vs Output Voltage, V 


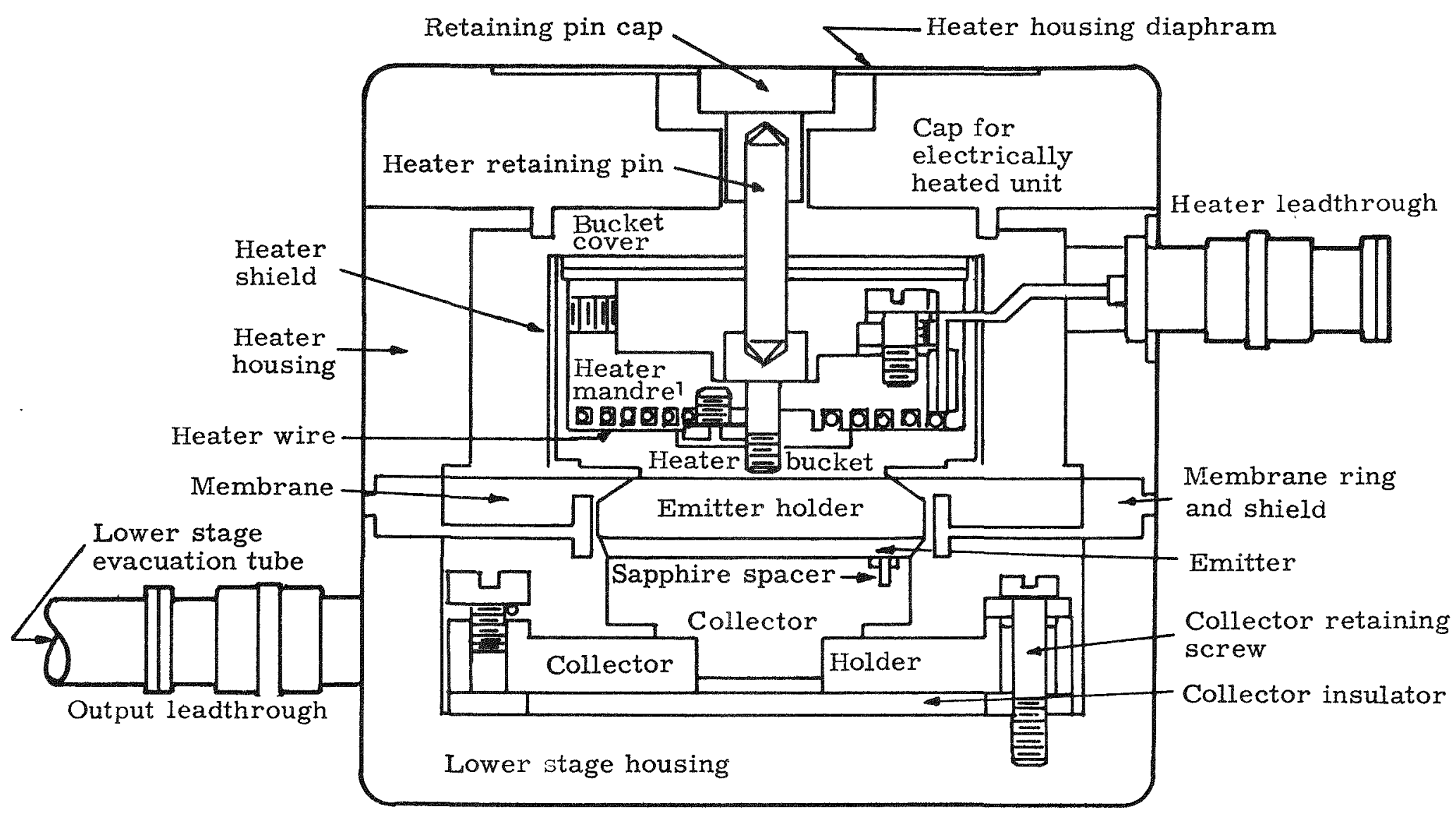

Fig. 24. Assembly No. 1-A, Electrically Heated Model 


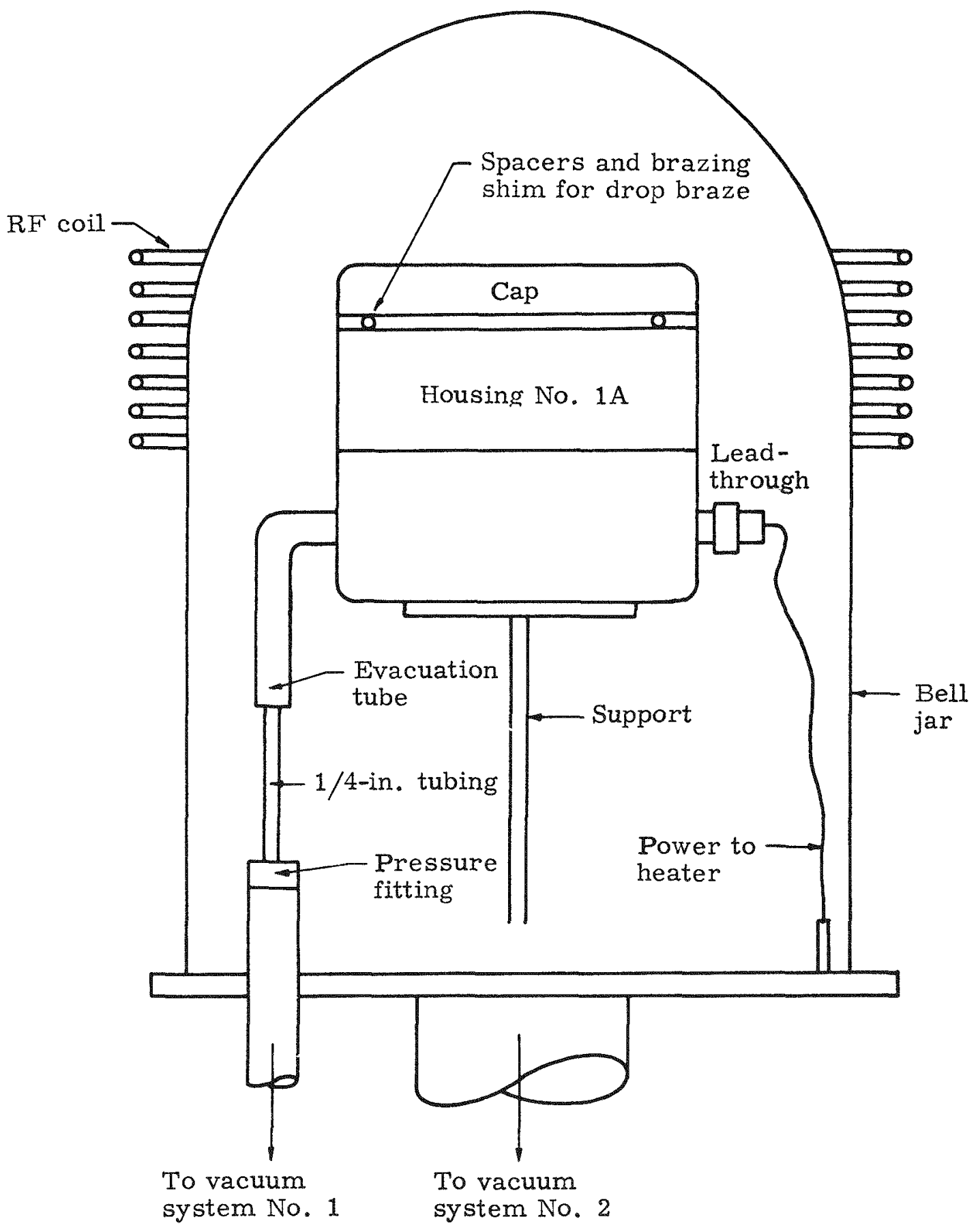

Fig. 25. Setup Used to Braze Housing 
ly due to uneven heating and partial melting of the brazing shim. The temperature was increased within the limits allowed by the leadthroughs $\left(1000^{\circ} \mathrm{C}\right.$ max.) but without effect. The unit was cooled to stop evaporation of $\mathrm{Ag}-\mathrm{Cu}$, which was causing high frequency discharges, endangering the bell jar.

Attempts to locally increase the temperature of the cap to melt the formed $\mathrm{Ti}-\mathrm{Cu}-\mathrm{Ag}$ eutectic were unsuccessful. A heavy molybdenum weight of suitable configuration to insure good RF coupling was placed on the cap. When the cap reached a temperature high enough to melt the braze, the weights forced it tight on the housing. Unfortunately, the excessive heat needed to accomplish this alloyed the platinum-10\% rhodium foil with the titanium, and a leak resulted.

Despite the fact that the unit was not vacuum-tight, it was tested under a bell jar. A weight acting on the platinum foil of the cup substituted for atmospheric pressure. The emission obtained was poor because of the poor vacuum inside the unit and the large spacing. Repeated exposure to the atmosphere and insufficient activation contributed to the poor emission.

The unit was then used to study temperature distribution while in operation. Holes $1 / 8$ inch in diameter were drilled in the casing to facilitate temperature readings. Emitter, getter and shield temperatures were 1100,800 and $916^{\circ} \mathrm{C}$, respectively。

\section{Heat Transfer Study and Tests}

There are two sources of contact surface heat resistance. One is between the emitter and the emitter holder and the other between the emitter holder and the heater. The second resistance is caused by the platinum-10\% rhodium foil which is placed between the holder and the heater. These resistances should be minimized to improve the heat transfer between the heater and the emitter.

The surface coefficient between emitter and holder was reduced by placing a thin film of molybdenum powder ( 325 mesh) on the holder before securing the emitter in place. This reduced the temperature difference between the two surfaces from $60^{\circ} \mathrm{C}$ to $20^{\circ} \mathrm{C}$.

To reduce heat losses from the heat source to the holder, the following procedure was followed. The holder was provided with a cup to accomodate the entire heater. A cap was used to close the opening after the heater was inserted. The heat radiated from the side and top of the heater was collected by the holder. Great care was taken to make the alumina coating on the active side as smooth as possible to 
increase the contact area for heat transfer. In addition, a molybdenum heat shield was placed around the entire cup assembly to reduce radiation losses.

A new heater has been designed which has the heat sources directly brazed to the emitter holder, thus eliminating one of the surface barriers. The wire is imbedded on the side of the heater opposite that of the emitter holder. This alumina-coated side of the heater is very carefully shielded to avoid excessive losses due to high thermal emissivity of the alumina. This heater is now under construction.

As mentioned previously, there is a temperature gradient between the emitter and the emitter holder. An apparatus was constructed to measure this gradient over a range of contact pressures. Figure 26 is a drawing of the apparatus.

The assembly includes a weight platform attached to the top of the collector heater and collector. Interelectrode separation is maintained by sapphire spheres. The emitter heater, holder and emitter rest in a molybdenum housing which supports the collector assembly. Contact pressure between the emitter and holder is a function of the weight placed on the platform.

The unit was first evacuated to a pressure of $10^{-6} \mathrm{~mm} \mathrm{Hg}$ under a bell jar on a 2 -inch vacuum system. All temperatures were measured with an optical pyrometer.

The emitter heater brought the holder up to an operating temperature of $2100^{\circ} \mathrm{F}$. At this temperature, various weights were placed on the platforms. The temperature difference, $\Delta \mathrm{T}$, between the emitter and the holder was then recorded as a function of the emitter contact pressure developed by the weights. Figure 27 shows the results of this experiment.

The experiment showed that, at a pressure of 8.0 psia, the temperature gradient between the emitter and emitter holder was only $30^{\circ} \mathrm{F}$. This indicated that the weights reduced the film resistance between the holder and the emitter by increasing the contact area of the two pieces.

It was found that the optical pyrometer temperature readings were not sufficiently accurate due to the dependency on spectral emissivities of molybdenum and tungsten which vary from specimen to specimen. Thermocouples were introduced into the same experimental setup and tests were run again to determine temperature difference, $\Delta T$, vs contact pressure. Results are shown in Fig. 28. 


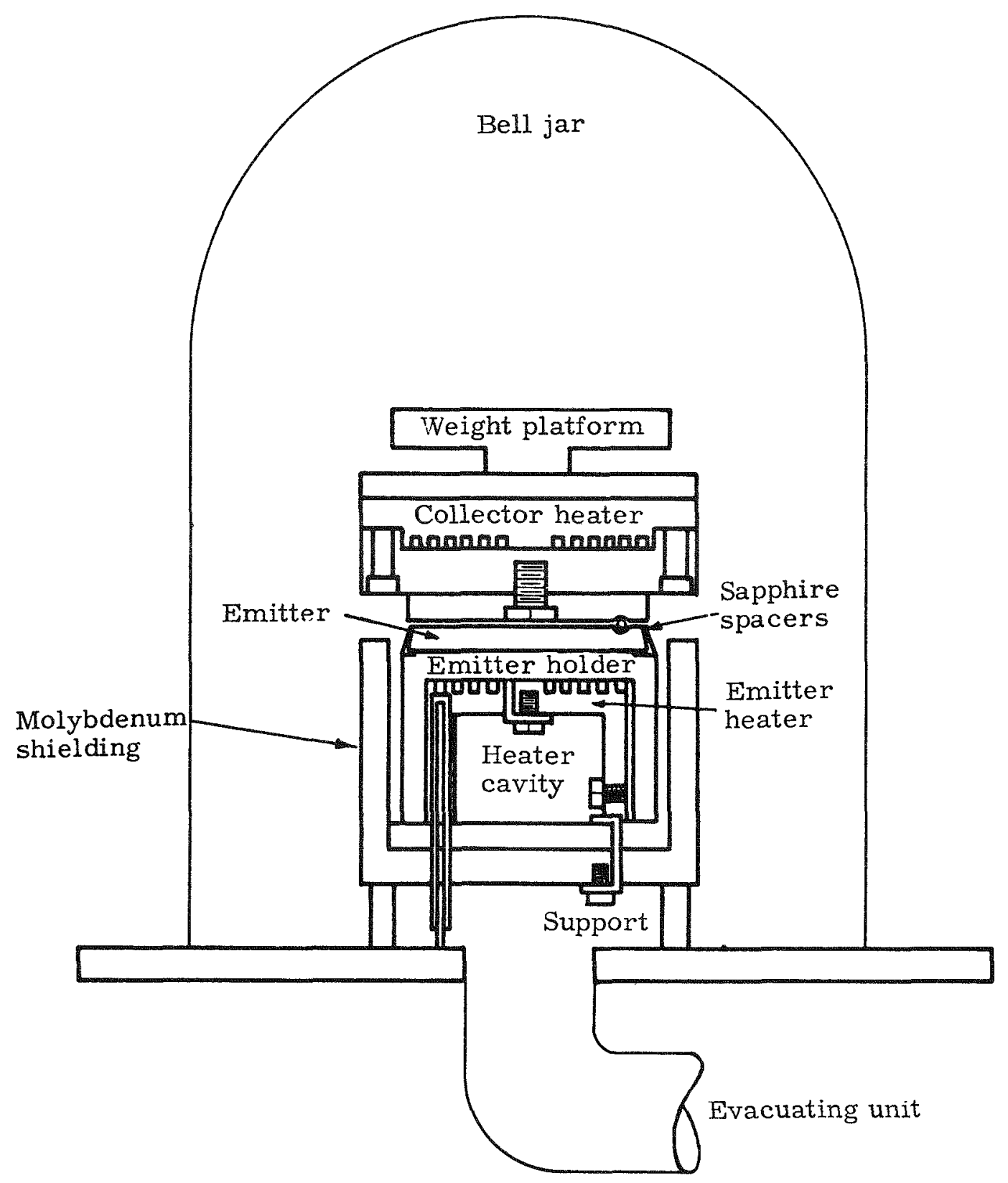

Fig. 26. Apparatus to Determine Heat Transfer from Emitter to Emitter Holder 


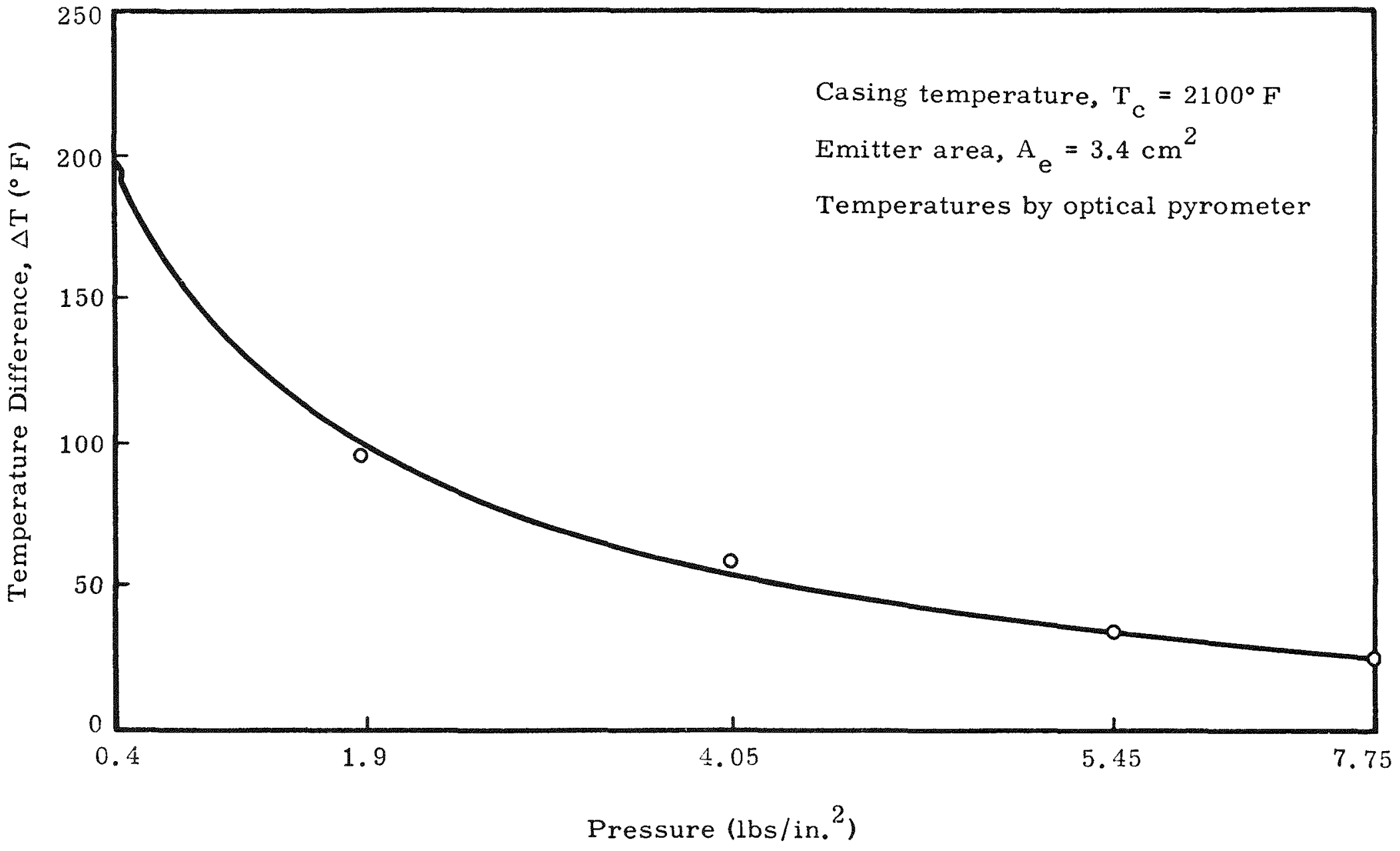

Fig. 27. Variation or Temperature Difference between Emitter and Emitter Holder with Pressure on Emitter 


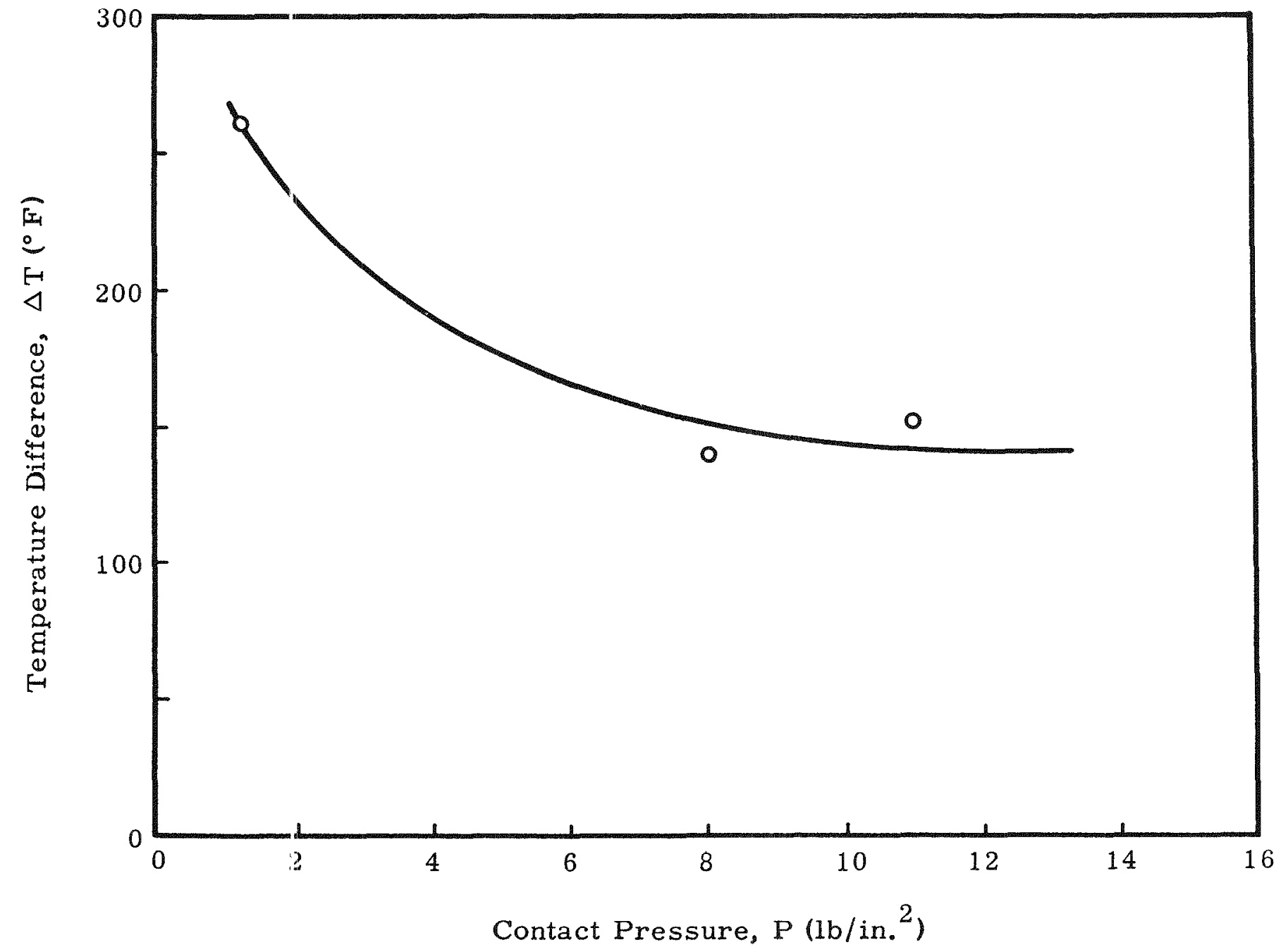

Fig. 28. Te.nperature Difference vs Contact Pressure Using Thermocouples 
Comparison of the results thus obtained with the ones obtained with the optical pyrometer shows the same behavior, but appreciable differences in exact value. $\Delta \mathrm{T}$ as measured by thermocouples is generally twice as great as that measured with a pyrometer. Readings were also taken while contact pressure was kept constant to see the variation of $\Delta T$ with casing temperature. The $\Delta T$ remains essentially constant with variation of casing temperature. An interesting phenomenon occurred during these experiments. Smaller $\angle \Delta T^{\prime} s$ were achieved as the temperature was decreased. This can be seen in Fig. 29. A possible explanation is that the contact area of the mating surface irregularities has increased as a result of the pressure and high temperature which produce some creep, thus increasing the heat conduction. However, this effect has to be investigated further before conclusions can be drawn.

As mentioned previously, the heat transfer from emitter holder to emitter may be improved significantly by coating the holder with a thin film of molybdenum powder. To improve the heat transfer further, a method of brazing the emitter to the holder must be developed.

A suitable braze must;

(1) Wet both tungsten and molybdenum.

(2) Not poison the emitter.

(3) Allow for mismatch of thermal coefficients of expansion.

3. Vacuum Tests in Prototype Shells

Titanium Casings. The shell is evacuated through the annular opening formed by spacers around the braze ring. These spacers are made of the same material as the braze to insure a tight seal (see Fig. 30).

A braze was performed in a vacuum bell jar with an $R F$ induction coil for heating. The ionization gage was connected to an amplifier to permit monitoring the pressure during the operation as well as to permit outgassing of the ion gage components.

An RF coil was also used to outgas the ionization gage. At the time of the braze, the pressure in the bell jar was about $10^{-5} \mathrm{~mm} \mathrm{Hg}$. Immediately after brazing, the pressure inside the shell was observed to be about $10^{-6} \mathrm{~mm} \mathrm{Hg}$. 


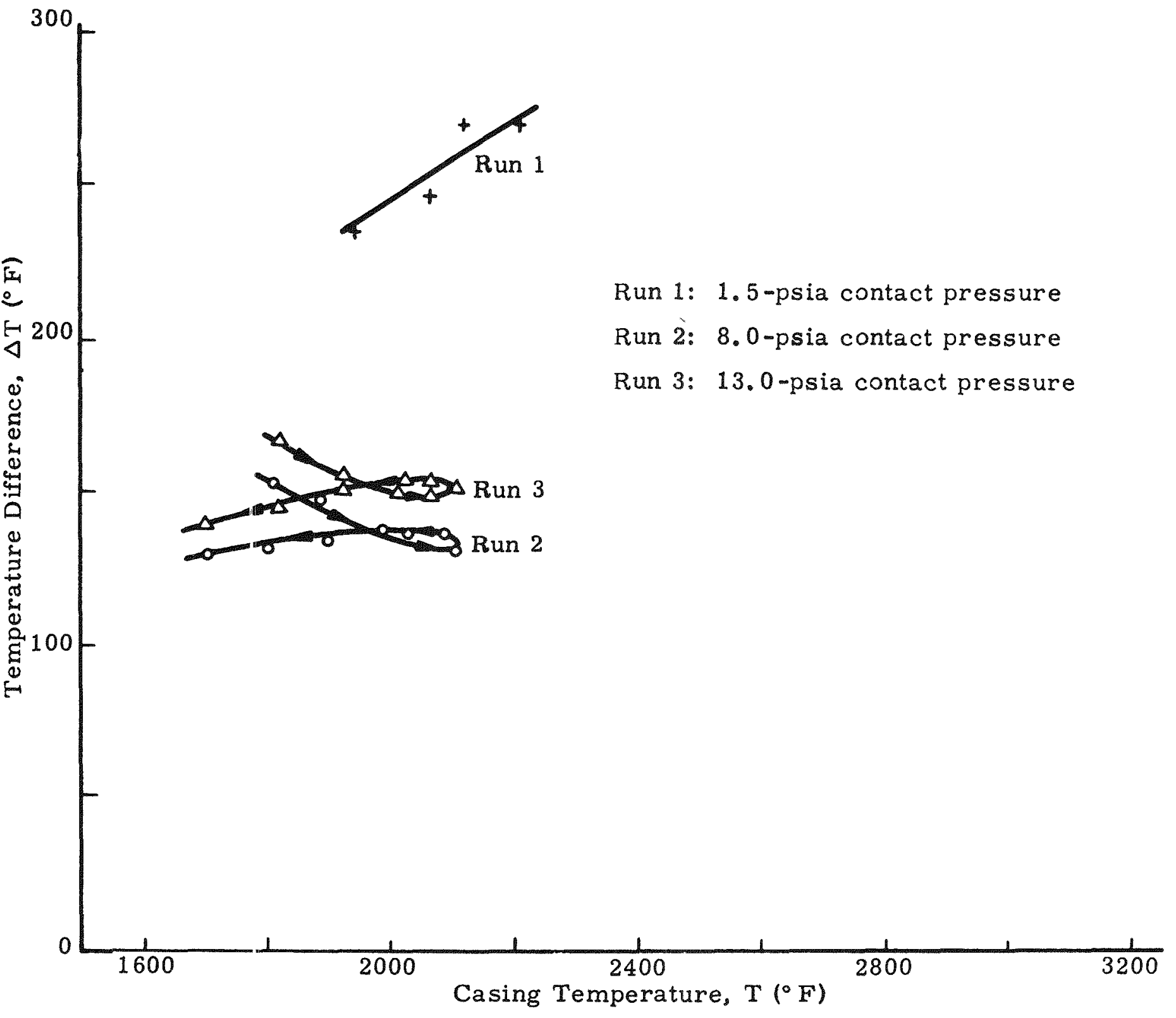

Fig. 29. Temperature Difference vs Casing Temperature 
To Kovar to glass seal, glass tubulation and ion gage

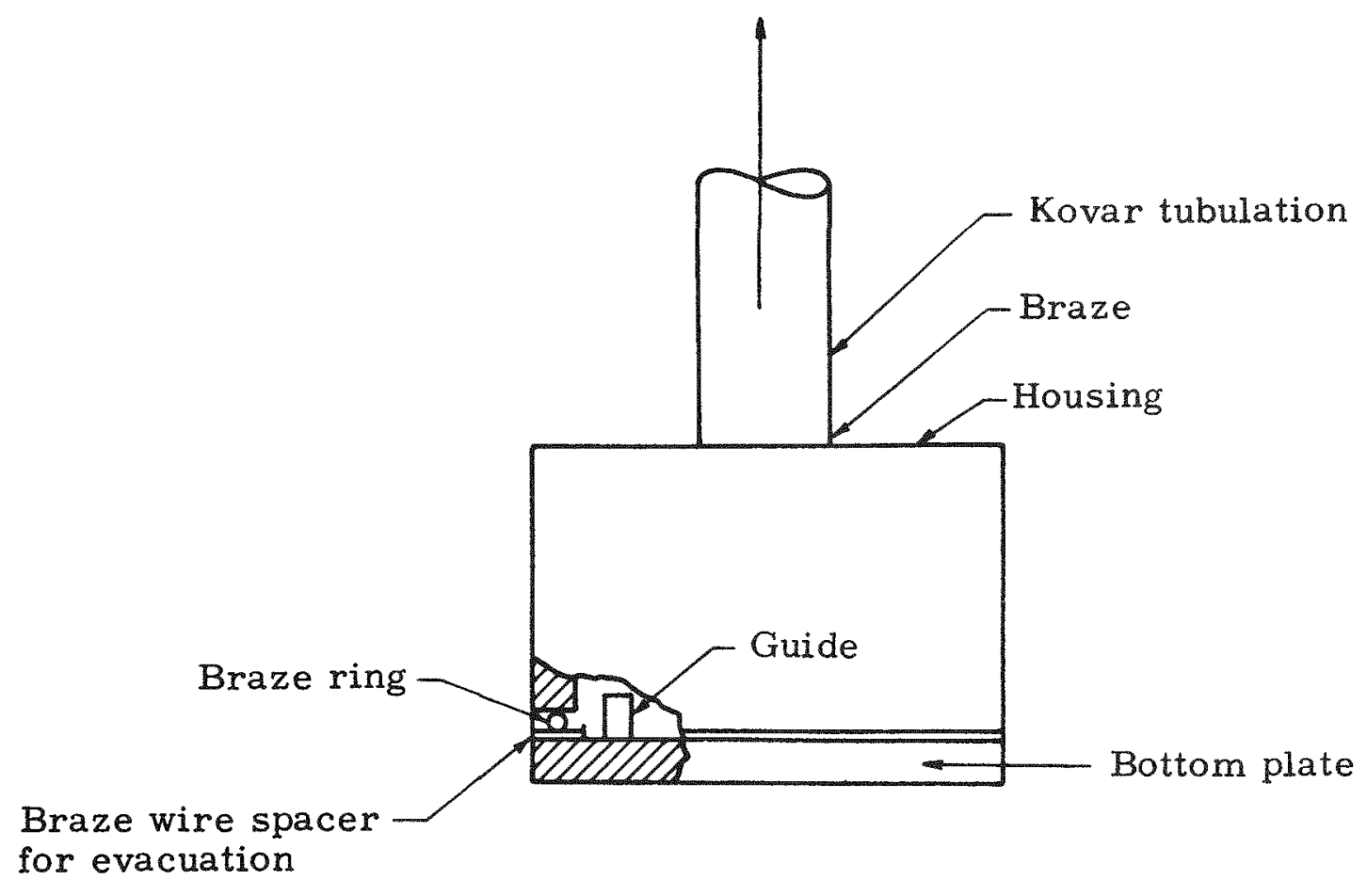

Fig. 30. Typical Shell for Evacuation Test

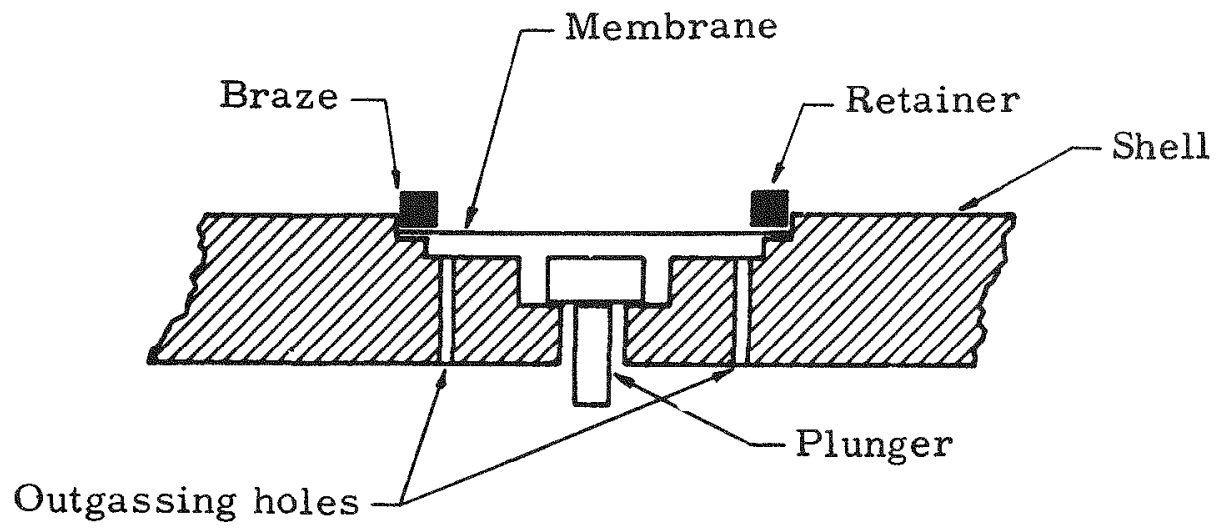

Fig. 31. Sketch of Membrane Detail 
Subsequently, the shell was heated by various means. A heating tape, bunsen burner, oven, and a hot plate were used to increase the rate of outgassing. Generally, it was found necessary to cool the ionization gage and its attachment to prevent overheating these components while the shell was being heated. Heating the unit with a gas flame for a few minutes produced a pressure of $6 \times 10^{-7} \mathrm{~mm} \mathrm{Hg}$.

The entire unit was later placed in an oven for 14 hours at a temperature of about $300^{\circ} \mathrm{C}$. At the end of this time, the pressure in the unit was higher than $5 \times 10^{-3} \mathrm{~mm} \mathrm{Hg}$. The application of a spark coil showed a black color. The loss of vacuum was attributed to the fact that the ion gage and associated kovar tube had been insufficiently outgassed during pressing, and gases were evolved during the bakeout.

Titanium casing with dummy leadthrough and pinched-off copper tubulation. This shell was similar in appearance to Shell no. 1, except a dummy leadthrough and a pinched-off evacuation tube were added.

Evacuation was accomplished through the joint where the final braze would be made. At the time of brazing, the pressure in the bell jar was $2.8 \times 10^{-6} \mathrm{~mm}$ of $\mathrm{Hg}$. After some time elapsed, the pressure inside the shell was $7 \times 10^{-7} \mathrm{~mm} \mathrm{Hg}$.

The ionization gage assembly was immersed in a water bath. The shell itself was heated by an electric hot plate to approximately $500^{\circ} \mathrm{C}$ for about 15 hours. At the end of this time, the gage was only partially immersed in the cooling bath and the amplifier was connected. The pressure in the unit was shown to be $1.2 \times 10^{-6} \mathrm{~mm} \mathrm{Hg}$ after 5 minutes. After heating for 15 minutes more, the pressure was found to be $2 \mathrm{x}$ $10^{-7} \mathrm{~mm} \mathrm{Hg}$.

When the ion gage was again completely immersed in the cooling bath, a hand torch was applied to the shell. Before heating with the torch, the pressure was recorded as $1.2 \times 10^{-6} \mathrm{~mm} \mathrm{Hg}$. Immediately after the heating was stopped, pressure was $3 \times 10^{-7} \mathrm{~mm} \mathrm{Hg}$. The amplifier was turned off. After about two hours, the amplifier was again turned on, showing a pressure of $3 \times 10^{-7} \mathrm{~mm} \mathrm{Hg}$.

It is concluded from these tests that the titanium shell acts as a vigorous getter when heated to a temperature of 400 to $600^{\circ} \mathrm{C}$. Unfortunately, pressures inside the unit cannot be measured while the shell is being heated due to the overheating of the glass and kovar parts associated with the gage. 
Titanium casing with metal ceramic leadthrough. Shell No. 3 was similar to No. 1 except that a leadthrough was brazed to the side of the shell. This unit was processed in the same manner as the other units. During brazing, the pressure in the bell jar was $2 \times 10^{-5} \mathrm{~mm} \mathrm{Hg}$, and the pressure inside the shell read $7 \times 10^{-4} \mathrm{~mm} \mathrm{Hg}$. When the unit was cooled to room temperature, the pressure was $3 \times 10^{-6} \mathrm{~mm} \mathrm{Hg}$. The shell was heated by a burner and the ion gage was immersed in a cooling bath. The lowest pressure observed after heating for 5 to 10 minutes was $6 \times 10^{-7} \mathrm{~mm} \mathrm{Hg}$.

Titanium casing with platinum membrane. To test the leak tightness of the membrane to be used in prototypes, a membrane of 0.002 -inch thick platinum-10\% rhodium foil was brazed to a titanium shell (see Fig. 31\%. A plunger was applied behind the membrane to act upon the inside of the shell. Suitable outgassing holes were provided in space between the membrane and the casing.

In the first attempt to braze this unit a copper evacuation pipe, which was pinched off at the end, was included. Overalloying of the copper took place due to excessive brazing temperature, resulting in a porous braze.

In the second attempt, the copper tube was removed and all the brazes were successfully completed. At the time of the braze, the bell jar pressure was $7 \times 10^{-6} \mathrm{~mm} \mathrm{Hg}$, and in the shell $1 \times 10^{-4} \mathrm{~mm} \mathrm{Hg}$. When the unit was cooled to room temperature, the shell pressure was $1 \times 10^{-6}$ $\mathrm{mm} \mathrm{Hg}$.

Titanium and monel shell. A shell was prepared in which a monel ring was sandwiched between two titanium cups, forming the rest of the shell. The parts have been prepared and are awaiting brazing.

CFI leadthroughs. A large-diameter CFI leadthrough was removed from a leaky unit and sectioned to facilitate physical examination. It was then evident that no copper brazing material was alloyed with or firmly attached to the metallized portion of the ceramic. The metal section could easily be removed from the ceramic part. It is not known whether the strain resulting from the physical removal of the leadthrough from the housing or the continued reheating of the ceramic caused its failure.

A smaller diameter CFI leadthrough was incorporated in a sealed housing containing an ionization gage. The unit was heated to a temperature of $1000^{\circ} \mathrm{C}$ for 10 minutes to braze several joints. After extensive testing, the housing proved to be vacuumtight. This indicated that the CFI leadthrough did not leak and could be considered reliable. 
TEECO metallized ceramic leadthrough. Six matched expansion metallized ceramic leadthroughs were constructed from thin-wall kovar cylinders and Raytheon R-95 ceramic washers, metallized with Ti-Mo metallizing compound. An OFHC copper braze joined the parts. The six units were tested on a differential absorption leak detector. One assembly leaked due to insufficient metallizing on the inner diameter of the ceramic washer.

A leakproof unit was examined by cross sectioning. Good bonding was evident. The thin-wall kovar tubing was then stripped off the ceramic. Considerable force was required to accomplish this separation. Metallization adhered well to both the ceramic and kovar, although no ceramic chips stripped off with the metal.

Another unit was repeatedly thermally cycled from ambient temperature to $900^{\circ} \mathrm{C}$. No leaks developed.

Initial tests indicate that the TEECO metallizing technique is quite successful. The process is lengthy, however, since the metallized ceramic pieces must be vacuum fired at $1500^{\circ} \mathrm{C}$ and nickel plated before brazing.

TEECO reactive metal leadthroughs. Four titanium, nickel shim and ceramic leadthroughs were designed and constructed. Two were made with 0.00075 -inch nickel shims and a tight fit between the ceramic and titanium. The second two were made with 0.003 -inch nickel shims. The titanium and ceramic were fitted loosely so that the nickel alloy would form fillets. Fillets did form, but one unit leaked. One unit was sectioned and showed good adherence of alloy, both to the ceramic and the titanium.

\section{Cathode and Sapphire Support Creep Tests}

Tests on prototype PT4 showed that the flexible collector support developed significant creep at the high temperatures necessary for brazing and outgassing. Therefore, the flexible support was changed to a rigid support which was brazed to the collertor with nirkel hraze. The collector assembly was held in position by alumina insulated screws and an alumina disc provided insulation from the housing.

The results reported in Section A.2. indicate that an increased pressure between two surfaces will improve the thermal conductivity across the film. However, this would increase the pressures transmitted by the sapphire rod spacers, which will aggravate the creep problem. Effort was initiated at the close of this quarter to determine the feasibility of increasing the contact pressure. 


\section{Parametric Study}

A parametric study was begun this quarter to lay the foundation for later isotopic-fueled thermionic generator designs. Information is sorely needed concerning the characteristics of the thermionic generator for different isotope power densities--the optimum power level, the efficiency, etc.

Considerable thought was given to the approach to be taken during the early part of the work. It became apparent that all parameters could not be considered equally, so the first task was to determine which parameters were paramount. It was decided that the power level, the emitter temperature, the efficiency, the design approach and the power density of the isotope were most important. It was further decided that the power density of the isotopic heat source would be used as the common abscissa and the power level as the common ordinate, with the other terms as vaariable parameters. These other parameters would then be shown over the possible ranges of interest. The analytical work is now underway generating points for such a graph(s).

\section{B. MARTIN NUCLEAR DIVISION EFFORTS}

The work performed by Martin Nuclear Division can be broken down for reporting purposes into the following categories:

(1) Development of Molybdenum Fuel Capsules.

(2) Heliarc Welding of Molybdenum Fuel Capsules.

(3) Hazards Report on Ce-144-Fueled Thermionic Unit.

(4) Fabrication of Containment Cask for Ce-144 Unit.

(5) Fueling of the Molybdenum capsule with the Ce-144 Fuel Pellets Fabricated by ORNL.

\section{Development of Molybdenum Fuel Capsules*}

In October, six molybdenum fuel capsules were fabricated for use in making practice heliarc welds and to determine the fabricability of these capsules. One of these capsules is shown in Fig. 32.

Eight additional molybdenum fuel capsules were fabricated. Five of these were sent to ORNL. ORNL personnel will use two or three of these to make practice welds with the Martin-furnished welder. One of the others will serve as the capsule to be fueled with Cerium-144 fuel pellets, and the remaining capsule(s) is a spare. 


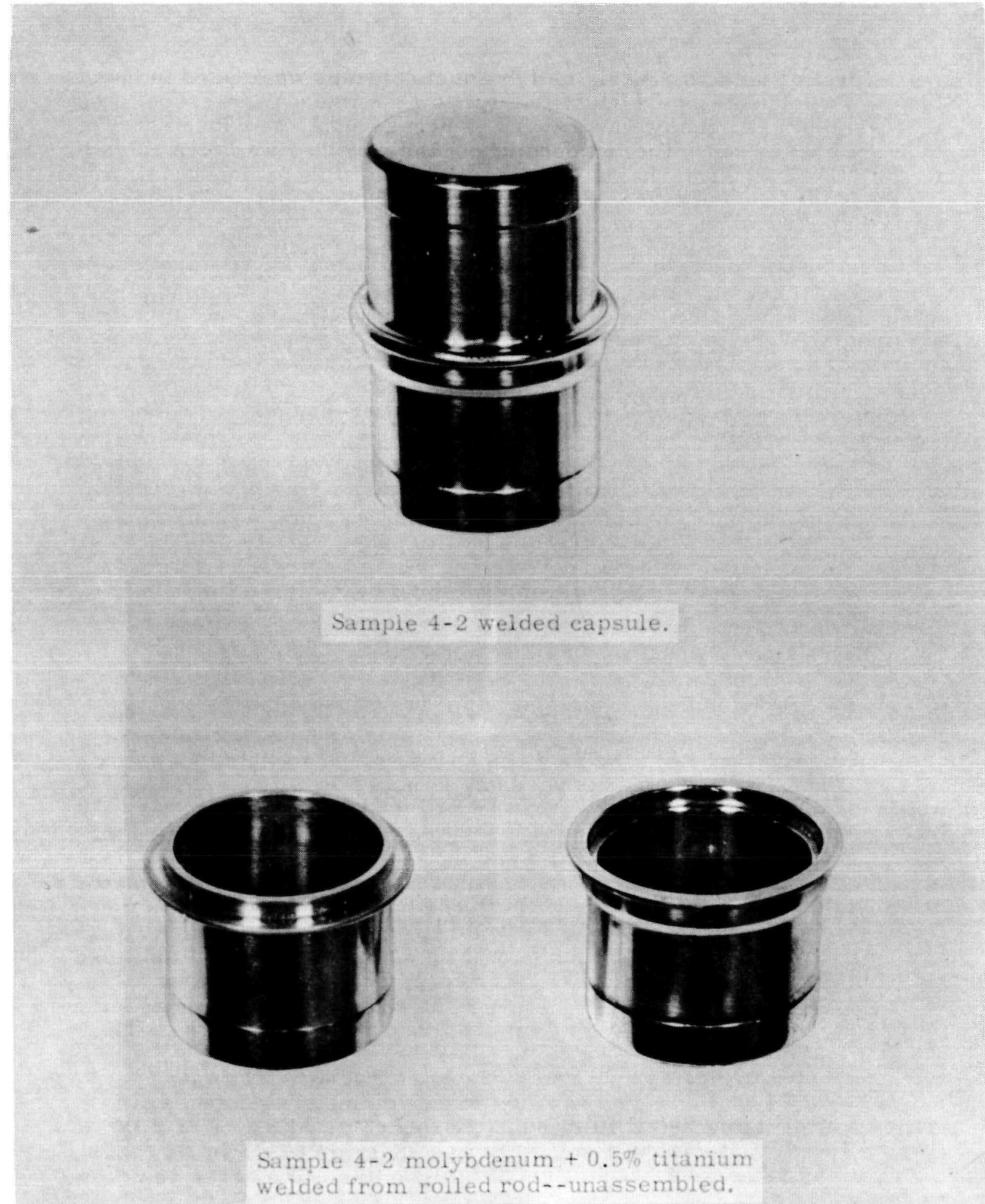

Fig. 32. Molybdenum Fuel Capsule 
Several of the original six molybdenum capsules were used in burst tests. The capsules were modified to allow the internal pressure tu be increased by introducing gas at various pressures. The capsules were then heliarc welded. The temperature of the capsules was then raised to $1700^{\circ} \mathrm{F}$ and the internal gas pressure raised in increments until the capsules failed. Pressures as high as 2200 psi were obtained before failure of the capsule occurred (see Fig. 33).

In two valid tests, the peripheral molybdenum weld failed in line with the parallel faces of the mating parts. One capsule came completely apart at the weld $\left(1600^{\circ} \mathrm{F}\right.$ at $\left.2000 \mathrm{psig}\right)$, while the other capsule $\left(1700^{\circ} \mathrm{F}\right.$ at $\left.2200 \mathrm{psig}\right)$ showed a split of about $3 / 8$ inch around the circumference where the gas pressure relieved itself. The molybdenum was ductile at this temperature, resulting in the expansion or bulging of the flat ends of the right cylinder by approximately 0.036 inch (Fig. 33).

One problem had to be solved before the burst test could be carried out. An adequate means had to be found of attaching a tube to the molybdenum capsule to permit raising the internal gas pressure in the capsule. The tube attachment had to be leakproof, able to withstand temperatures in the neighborhood of $1700^{\circ} \mathrm{F}$, and inherently stronger than the capsule weld. After attempts to braze stainless steel tubing to the molybdenum capsule failed, the use of molybdenum tubing was investigated. This approach was successful because a molybdenumto-molybdenum weld could be used.

Better data on the thermal coefficient of expansion of ceric oxide indicated that the expansion of fuel pellets in going from low temperature during the fueling to the operating temperatures would be approximately double the expansion of the molybdenum fuel capsule. This would rupture the fuel capsule. Therefore, it was decided to put a modified Belleville spring in series with the two fuel pellets to absorb the differential expansion. Inquiries were made to locate a spring manufacturer who could provide a spring that would operate satisfactorily at the high temperatures to be encountered in the fuel capsule. Manufacturers of ceramic as well as metallic springs were contacted. However, all vendors contacted considered it a development item, and were reluctant to commit themselves. Therefore, The Martin Company attempted to develop a spring for use in the ceric oxide fueled capsule. Tantalum-tungsten alloys were first examined as the spring material.

A satisfactory spring requires a material that will retain its elasticity at $2700^{\circ} \mathrm{F}$. A series of seven experiments was made. All samples were subjected to an argon atmosphere in a furnace at $2700^{\circ} \mathrm{F}$, and the spring material was subjected to a 35 -gram load. In all cases, the material failed to retain its elastic properties. The seven samples were as follows: 


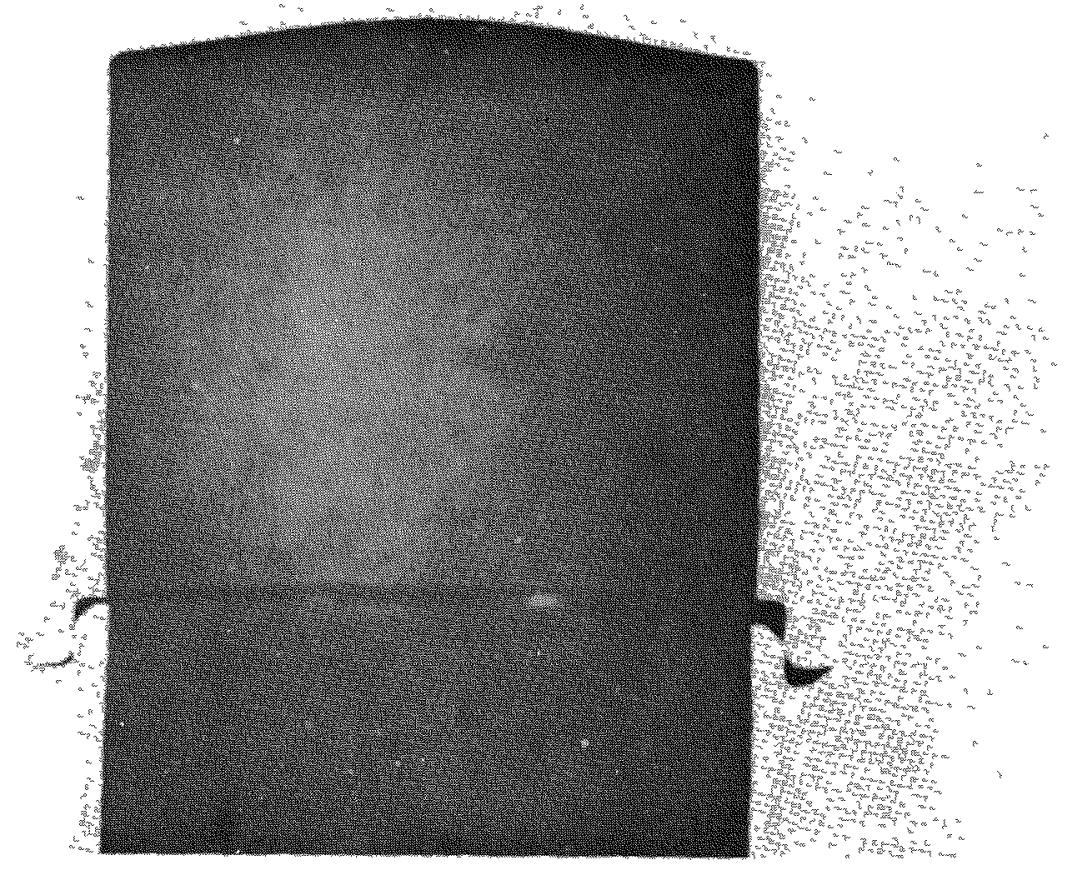

a. Capsule at Magn $3 \mathrm{X}$

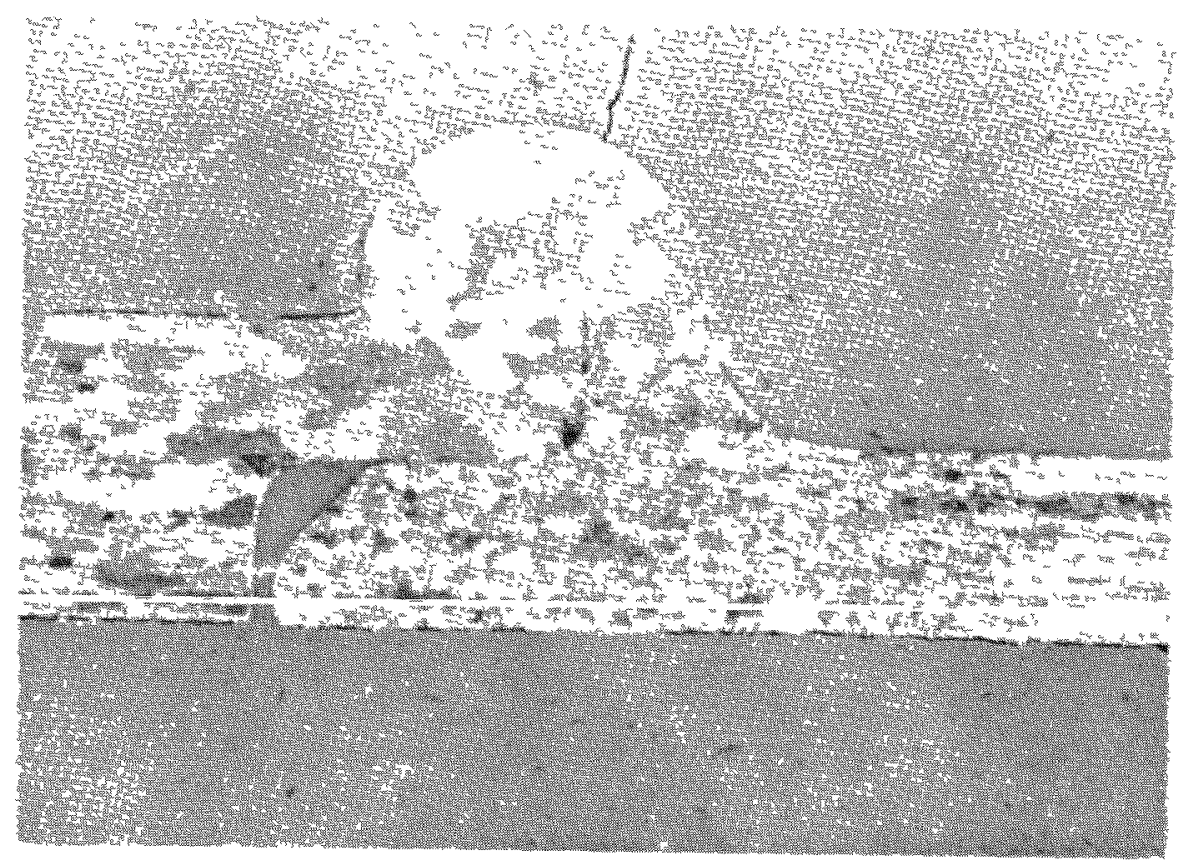

b. Micrograph of Burst Test Capsule Weld

Fig. 33. Burst Test Capsule 
(1) $7.5 \%$ tungsten $-92.5 \%$ tantalum, 0.012 inch thick, original deflection 0.032 inch.

(2) $10 \%$ tungsten-90\% tantalum, 0.023 inch thick, original deflection 0.041 inch.

(3) $100 \%$ tungsten, 0.005 inch thick, original deflection 0.036 inch.

(4) The same as (3), except rectangular-shaped spring instead of cross type.

(5) $10 \%$ tungsten-90\% tantalum, chemically etched to a thickness of 0.007 inch, original deflection 0.032 inch.

(6) $7.5 \%$ tungsten $-92.5 \%$ tantalum, 0.0115 inch thick. Material was given a special heat treatment at the suggestion of the supplier, Fansteel, original deflection 0.032 inch.

(7) Same as (6), except the sample was etched to a thickness of 0.007 inch.

The results of a typical run can be seen in Fig. 34 .

Separate tests have not been conducted to determine the effects on the molybdenum capsule of oxygen generation from the ceric oxide fuel. However, the various tests that have been conducted have shown that the molybdenum fuel capsule as designed, fabricated, and welded is a suitable container for a Cerium-144 heat source. The work is now complete.

\section{Heliarc Welding of the Molybdenum Fuel Capsule*}

Welding fixture. Modification of the welding fixture continued in order to simplify the remote welding of the fuel capsule and the shell of the thermionic unit. An electrode positioning mechanism was considered that would permit positioning the electrode relative to the piece to be welded before the bell jar was lowered into place. This positioning mechanism would also permit adjustments during the welding operation. Because of schedule pressures and the additional complexity of such a mechanism, a compromise design was selected in which the positioning mechanism for the welding electrode extends through the side of the bell jar. An experimental run verified the proper functioning of this mechanism. This modification was then incorporated in the welder. 


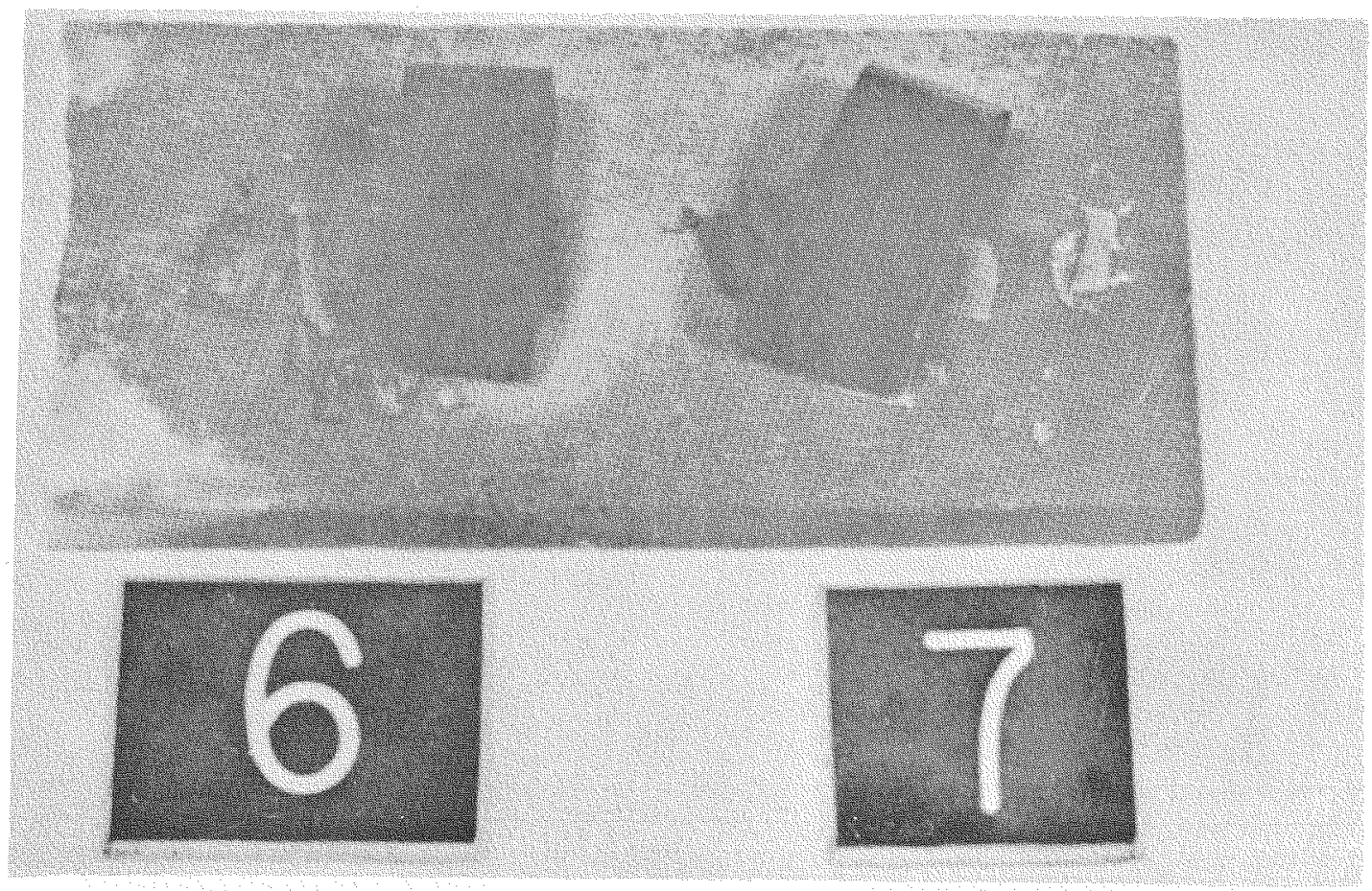

a. Sample with Weight

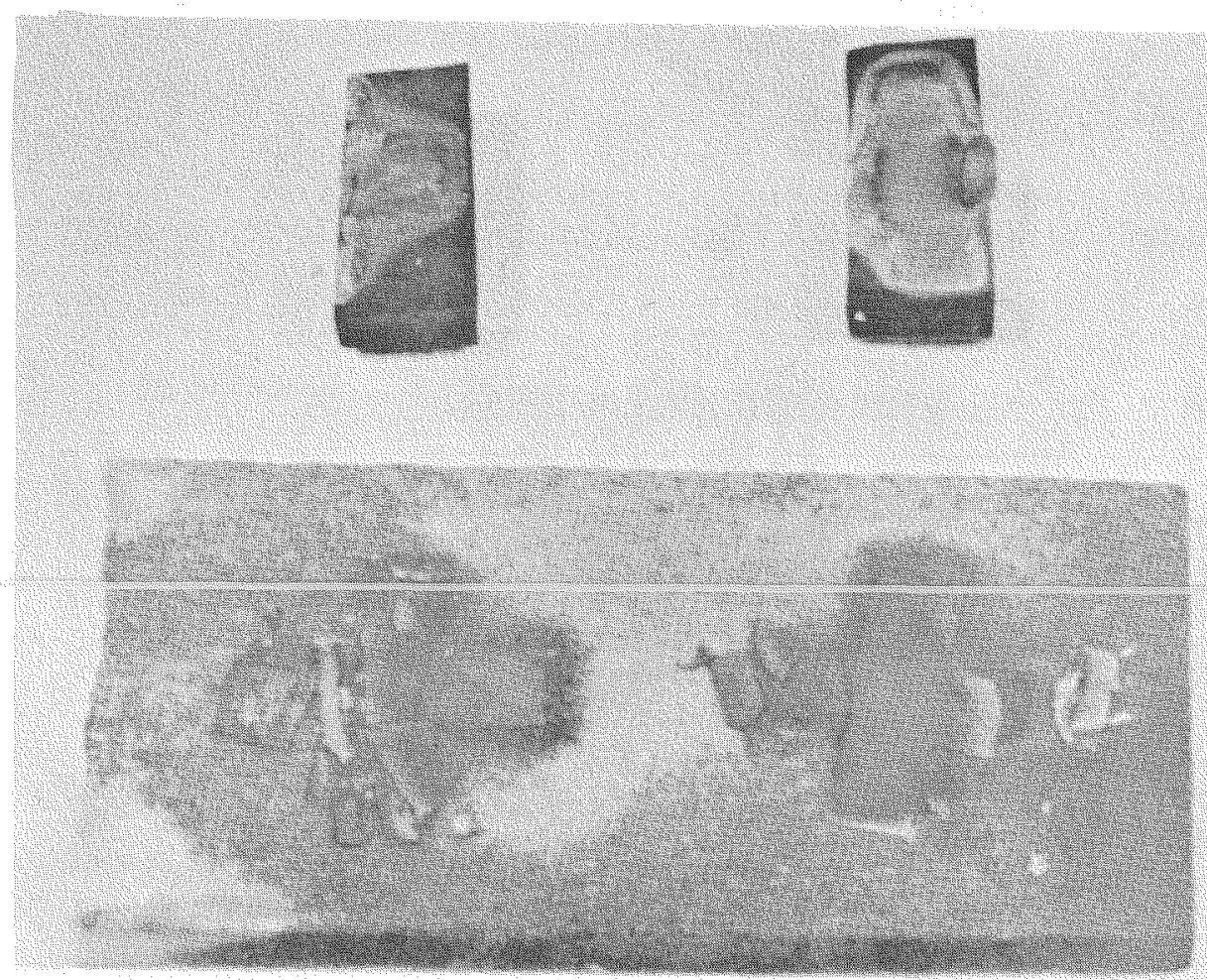

b. Sample with Weight Removed

Fig. 34. Typical Spring Sample 
All necessary parts were received for installing safety plate glass ports in the welder in lieu of quartz ports. This modification necessitated the design and fabrication of sight glass adapters.

Sufficient test runs were made to show that it is not necessary to fire a titanium getter in the welding fixture to obtain a satisfactory vacuum. It is sufficient to evacuate and backfill with a purge gas for three cycles before each weld.

The torch mechanism and chill blocks were completed, and were installed in the welder along with the sight glasses and adapters. The completed welding fixture is as shown in Figs. 35 and 36.

A smaller welder (see Figs. 37 and 38 ) was shipped to ORNL to familiarize the ORNL personnel with the remote welding of the molybdenum fuel capsules. The scheduled loading of the Ce-144 fuel pellets into the molybdenum fuel capsules was delayed at least six weeks due to the unavailability of hot cell facilities at ORNL. However, during the visit to ORNL, sufficient orientation was accomplished that, when the hot cell facilities become available, the fueling can get underway without requiring the presence of Martin personnel.

ORNL found that the clamps provided for sealing the welder were not a practical hot cell device. The welder is being modified by ORNL, to provide a toggle clamp arrangement--a simpler hot cell operation.

Welding techniques. Significant progress has been made in developing the technology required to produce a leak tight, structural weld on the molybdenum fuel capsule. Sample molybdenum washers were heliarc-welded to determine the best helium pressure, arc-gap, and current. Figure 39 shows a sample washer.

Work continued in evaluating the molybdenum welding techniques to determine the parameters most significant in producing good welds. The welds obtained to date have been leak tight. However, a large grain size is produced in the weld zone which raises some doubt as to the relative strength of the weld and its ability to resist corrosion. Since the weld should be both leak tight and structurally sound, work was concentrated on obtaining a weld with smaller grain size, while preserving the leak tight characteristics of the weld.

Three types of tests for determining the structural strength of the molybdenum welds have been used. The first method, and the most conventional, consists of drawing strap specimens which have been buttwelded together in tension until failure occurs. The second method consists of sectioning welded washer specimens and drawing these in direct 


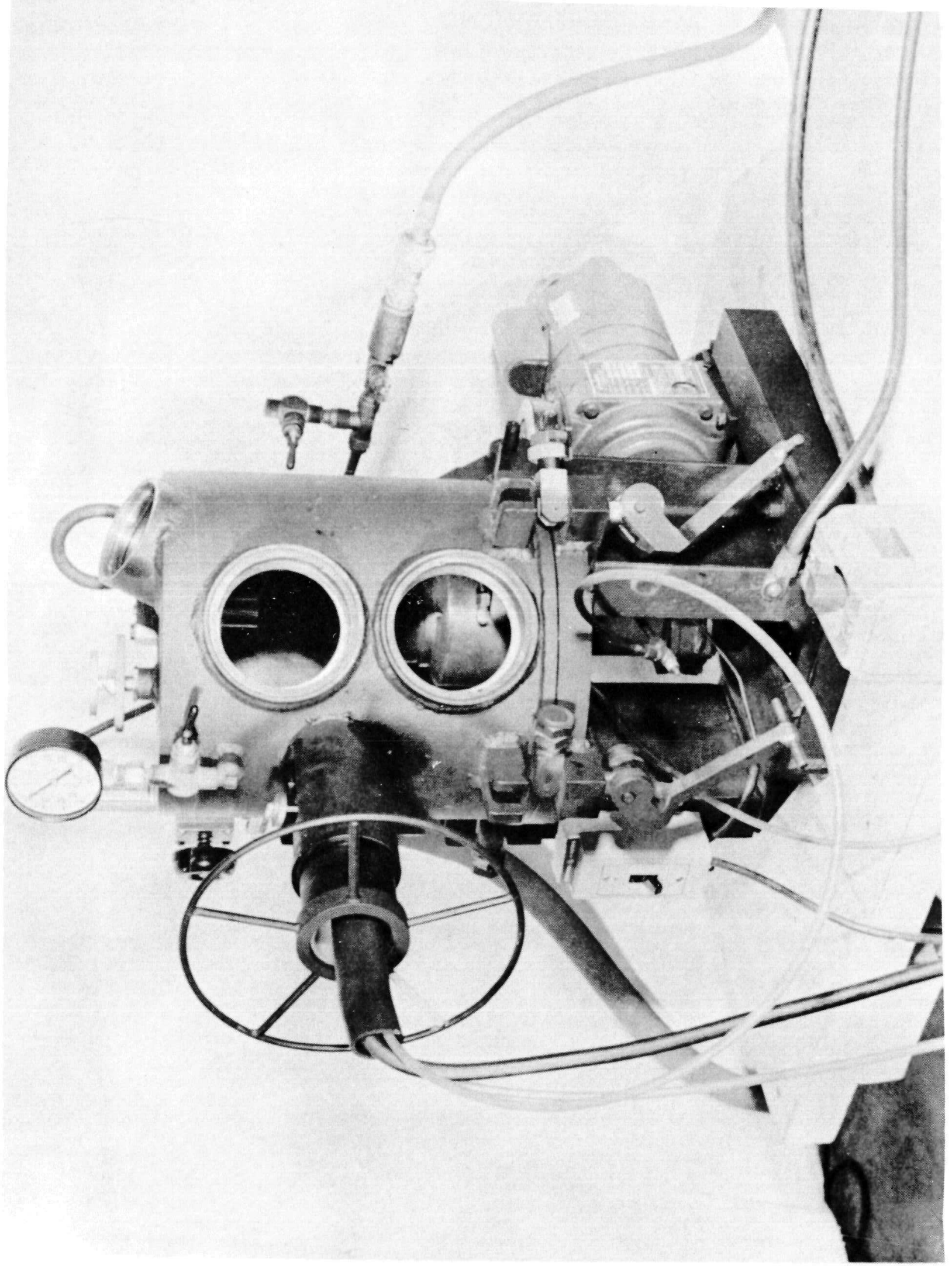

Fig. 35. Welding Fixture 


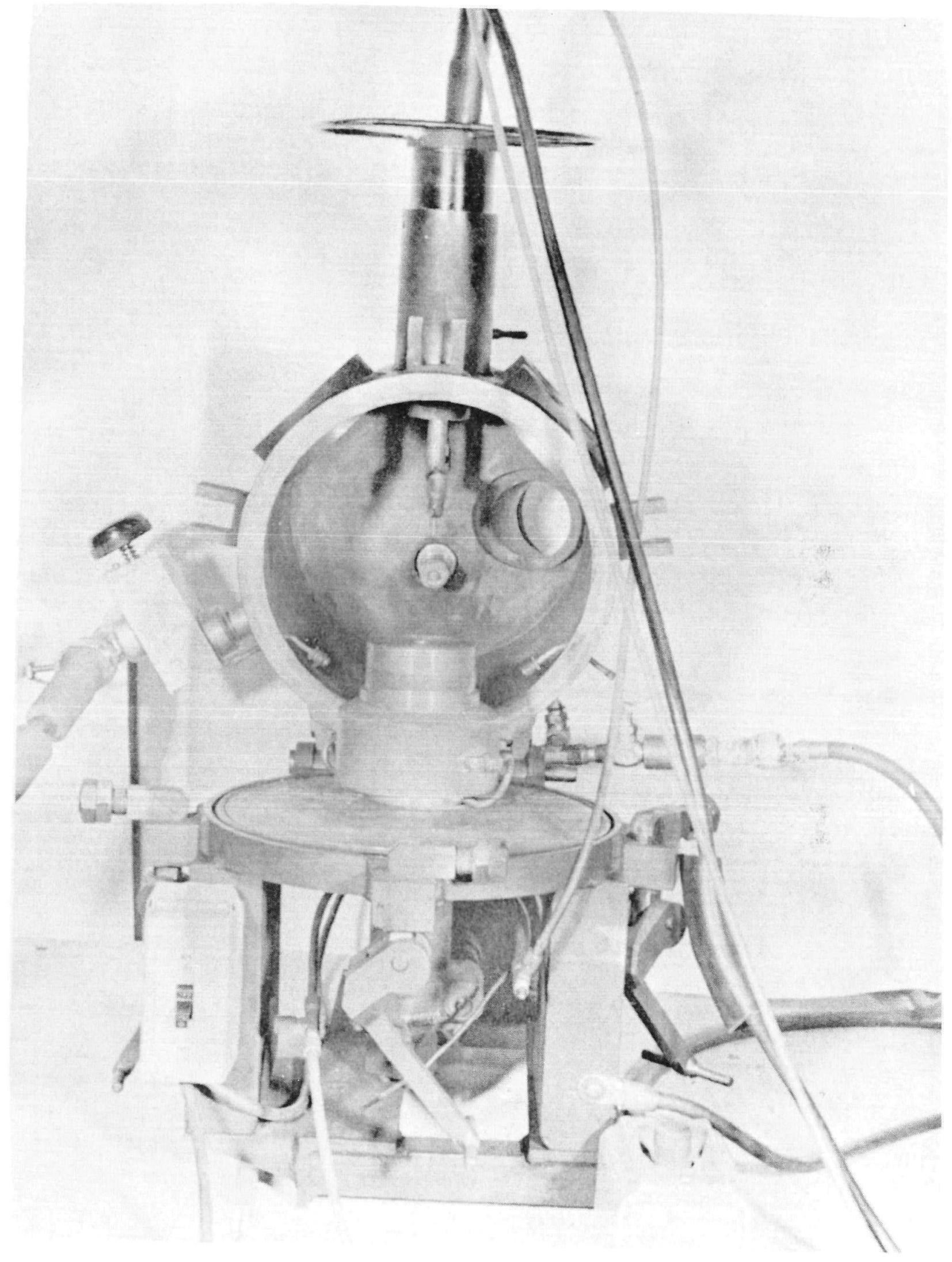

Fig. 36. Welding Fixture, Open 


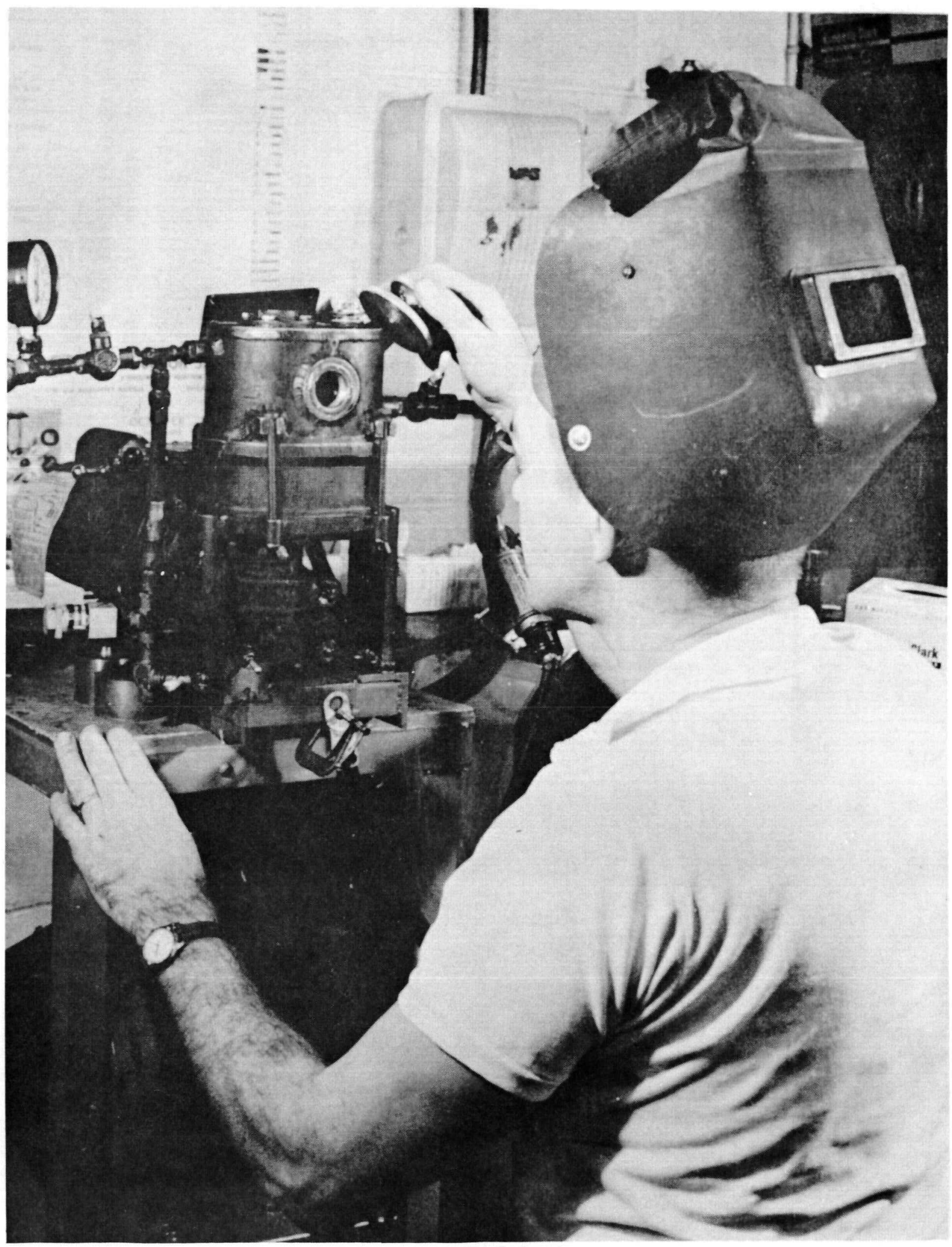

Fig. 37. Small Welding Fixture 


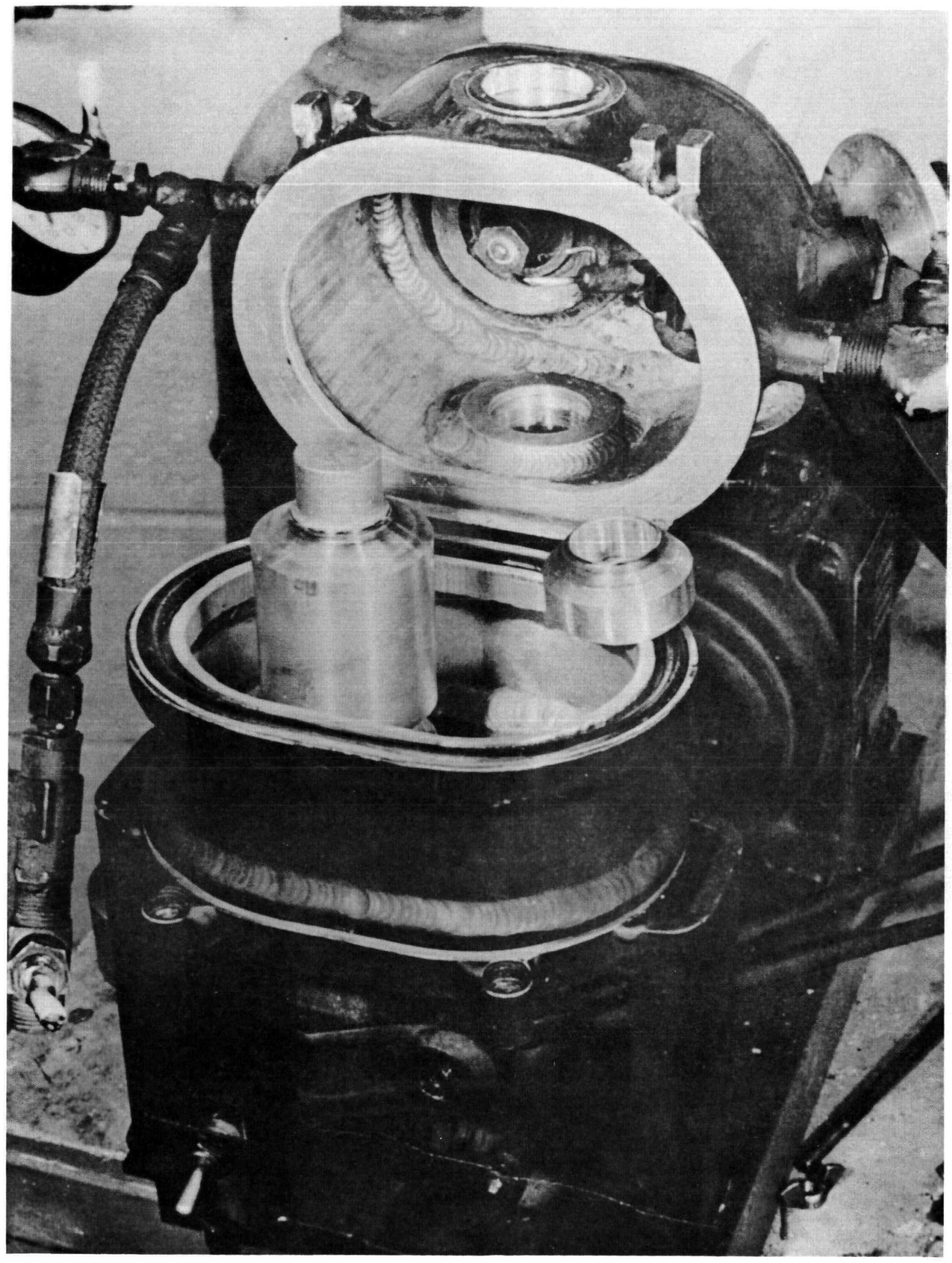

Fig. 38. Small Welding Fixture, Open 


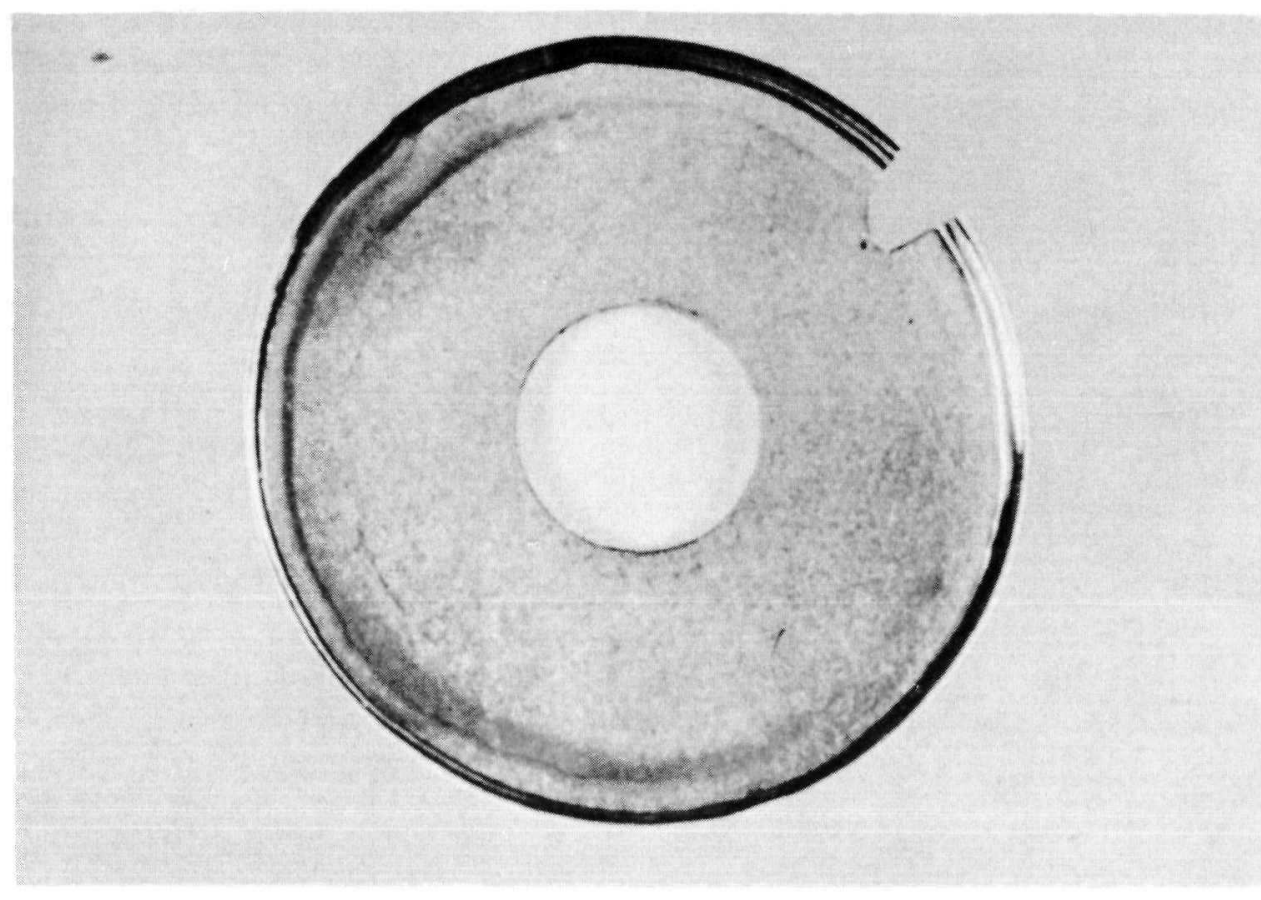

Fig. 39. Washer Welding Sample

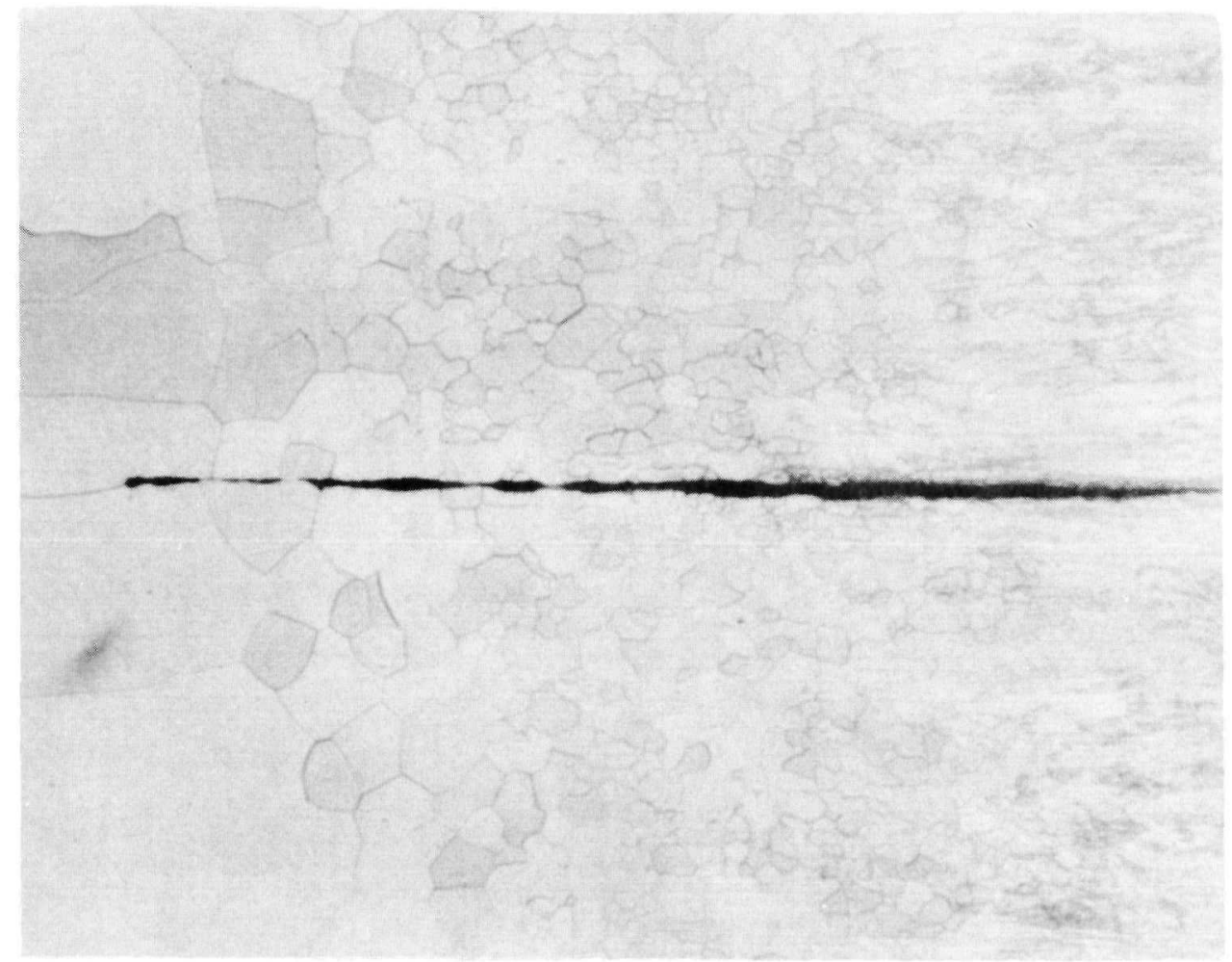

Fig. 40. Heat Affected Zone of Molybdenum Disc R-7, Welded at 90 amps in 150 psi He--Magn $75 \mathrm{X}$ 
shear. The third method, utilizing the same type of washer specimens, consists of drawing the weld in tension in the same direction the weld section of the capsule would be drawn due to an increase in internal pressure. All three methods were carried out at room temperature. These tests revealed that the heat-affected zone was generally weaker than either the weld area or the parent hot rolled molybdenum. This confirms reports in the literature.

A possible explanation for this weak heat-affected zone is the presence of nitrides which were observed in the intergranular areas. The nitrides are present in the parent molybdenum in minute quantities. The temperature-time history in the heat-affected zone may be responsible for concentration of the nitrides and resulting weakness. (See Fig. 40.)

A limited effort was made to evaluate the tensile data of the molybdenum weld samples. However, due to the small number of samples and the erratic results obtained, no definite conclusions can be drawn. The point of fracture varied from sample to sample; some weld fractures were intergranular while others were transgranular. Samples where the grain orientation was considered also yielded erratic results. Available time and funds do not permit the effort required to explore this behavior fully.

Within these limitations, all that can be said is that the weld is sufficiently strong for the present application.

The molybdenum capsule is composed of two halves, with the mating surfaces approximately $0.030 \mathrm{in}$. thick. The weld penetration was generally about equal to the lip thickness. (See Fig. 41.) This penetration would have been satisfactory except that, in many cases, the large grains extend from the root of the weld to the surface. (See Fig. 42.)

It was found that varying the helium pressure in the welding fixture had an effect on the grain size. Welds were made at pressures of 20 $\mathrm{Hg}, 50$ psig, 100 psig and 150 psig. The results are as shown in Figs. 43 through 46, respectively. Notice that the grain size reached a minimum at $50 \mathrm{psig}$, and then increased in size with increasing pressure.

As work progressed, the heavy black soot that formed during each weld cycle became more and more troublesome. The sooting actually darkened the sightglasses, contaminated the welding electrode, and formed a heavy deposit on the molybdenum material. The deposit was rich in molybdenum, but also contained traces of almost every material used in the fabrication of the welder. Workers with the heliarc welding 


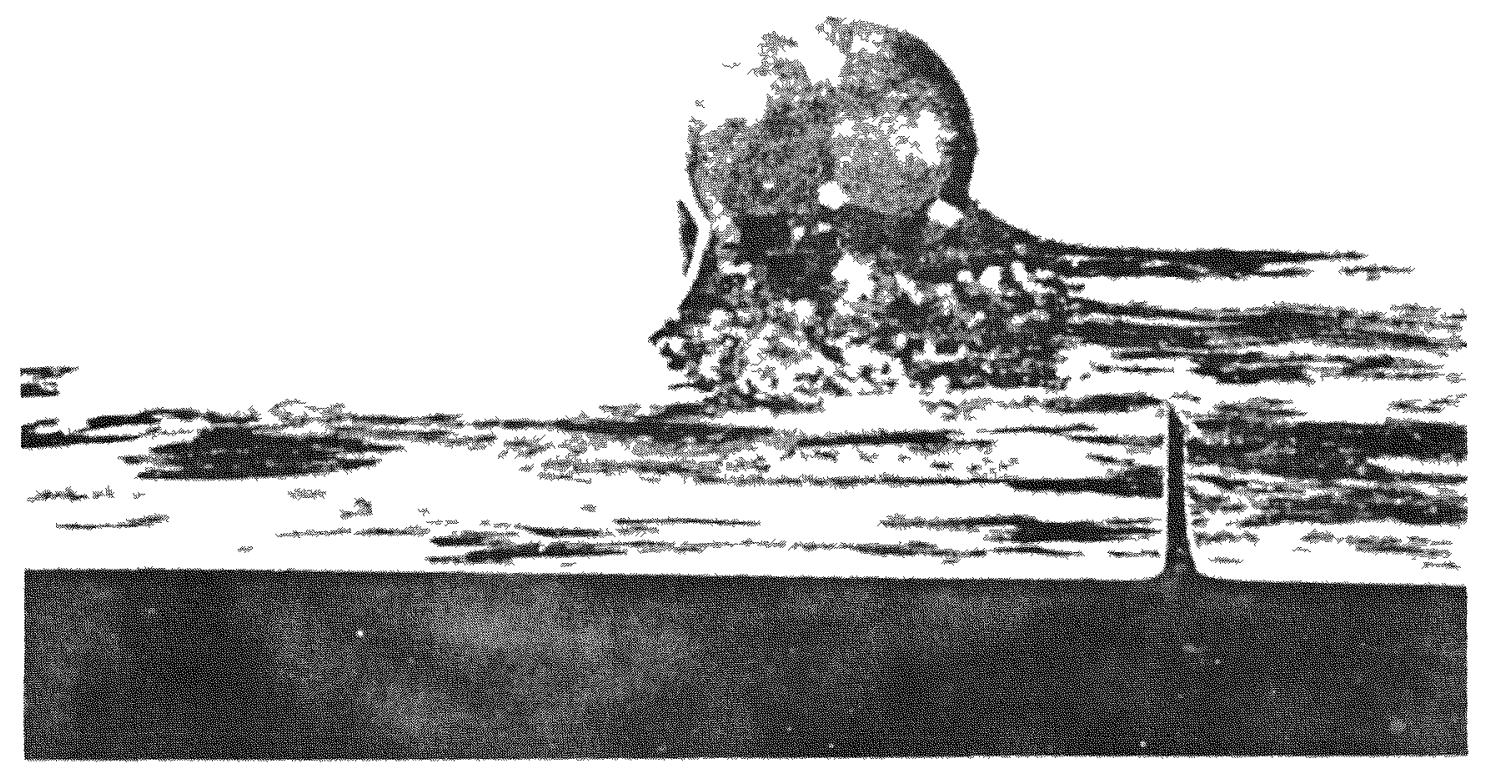

Fig. 41. Weld Area of Capsule Welded at 120 amps in 1 Atmosphere of He-Magn 10X
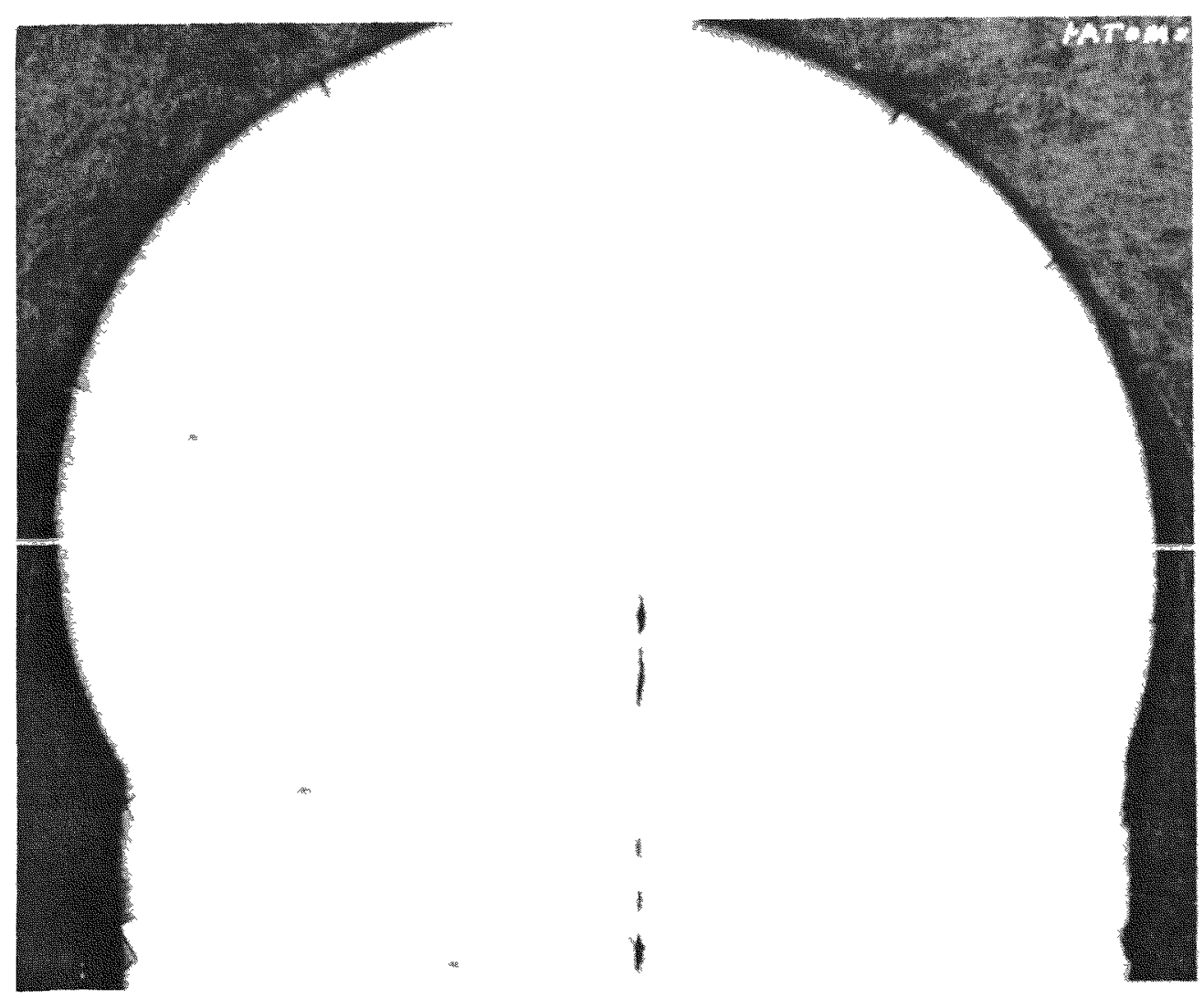

Fig. 42. Weld Zone of Molybdenum Disc R-1, Welded at 90 amps in 1 Atmosphere of He--Magn $75 \mathrm{X}$ 


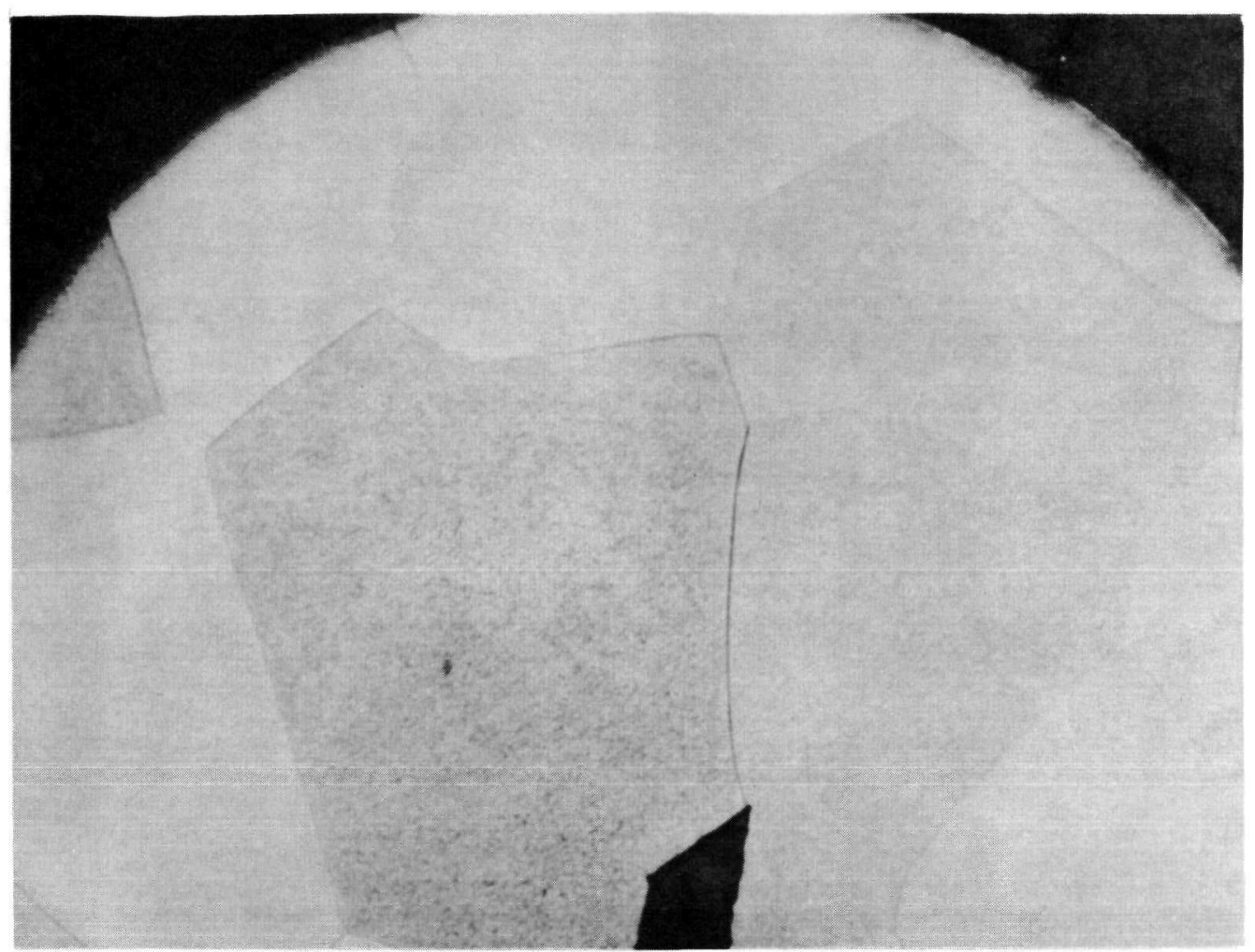

Fig. 43. Weld Zone of Molybdenum Disc R-1A, Machine Welded at 90 amps in 20-Inch Vacuum--Magn 75X

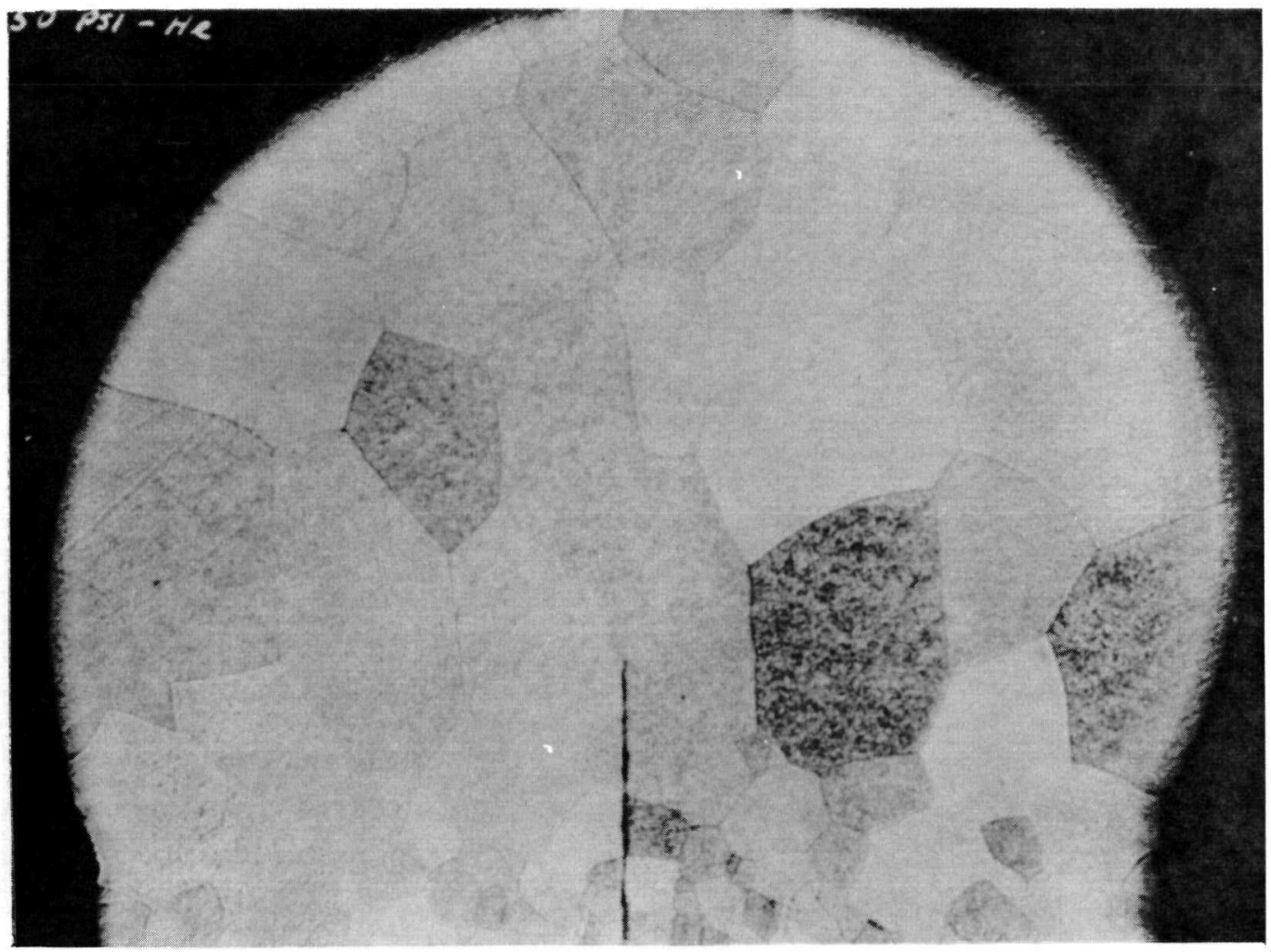

Fig. 44. Weld Zone of Molybdenum Disc R-3, Welded at 90 amps in 50 psi He--Magn $75 X$ 


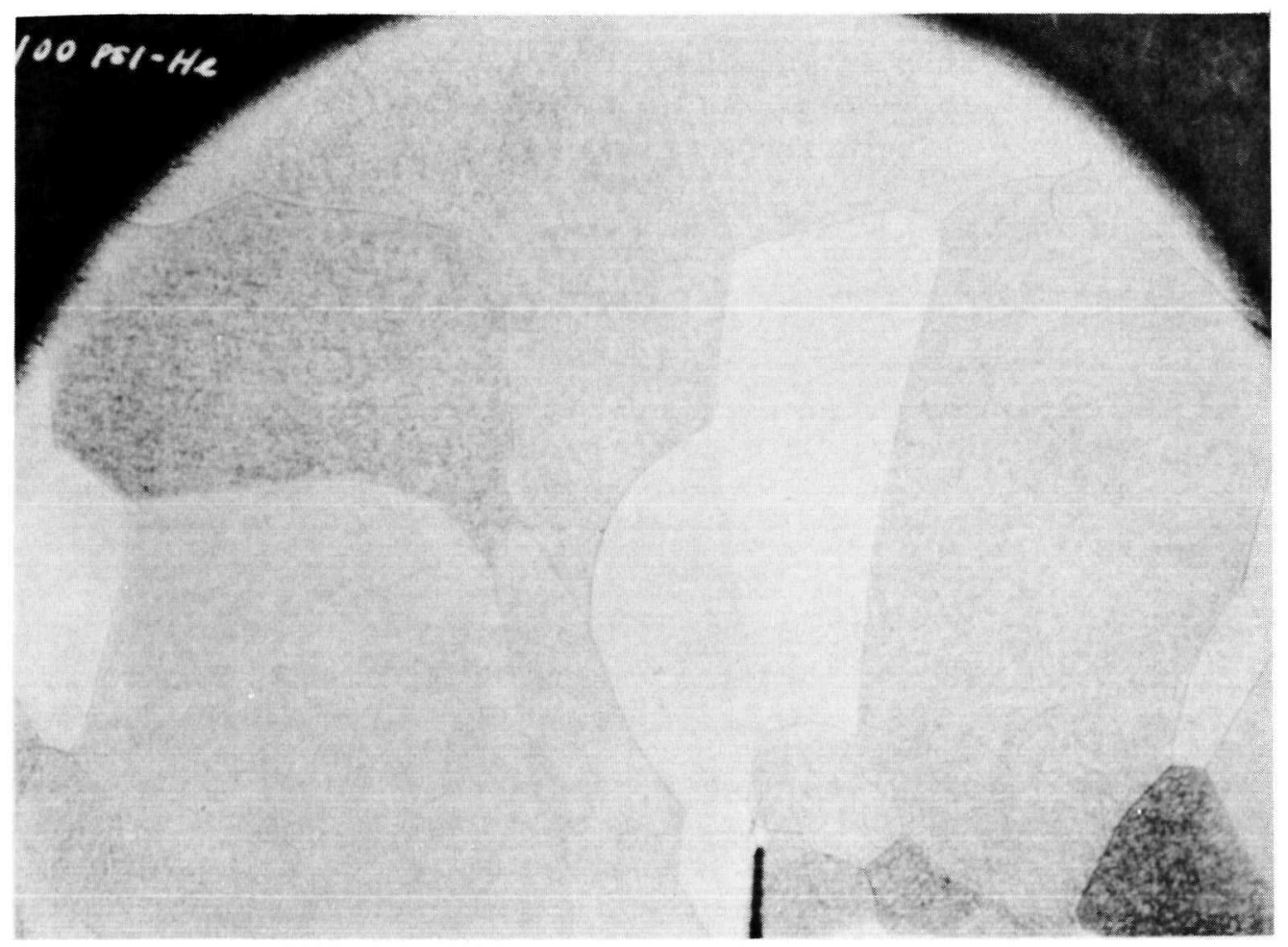

Fig. 45. Weld Zone of Molybdenum Disc R-5, Welded at 90 amps in 100 psi He--Magn 75X

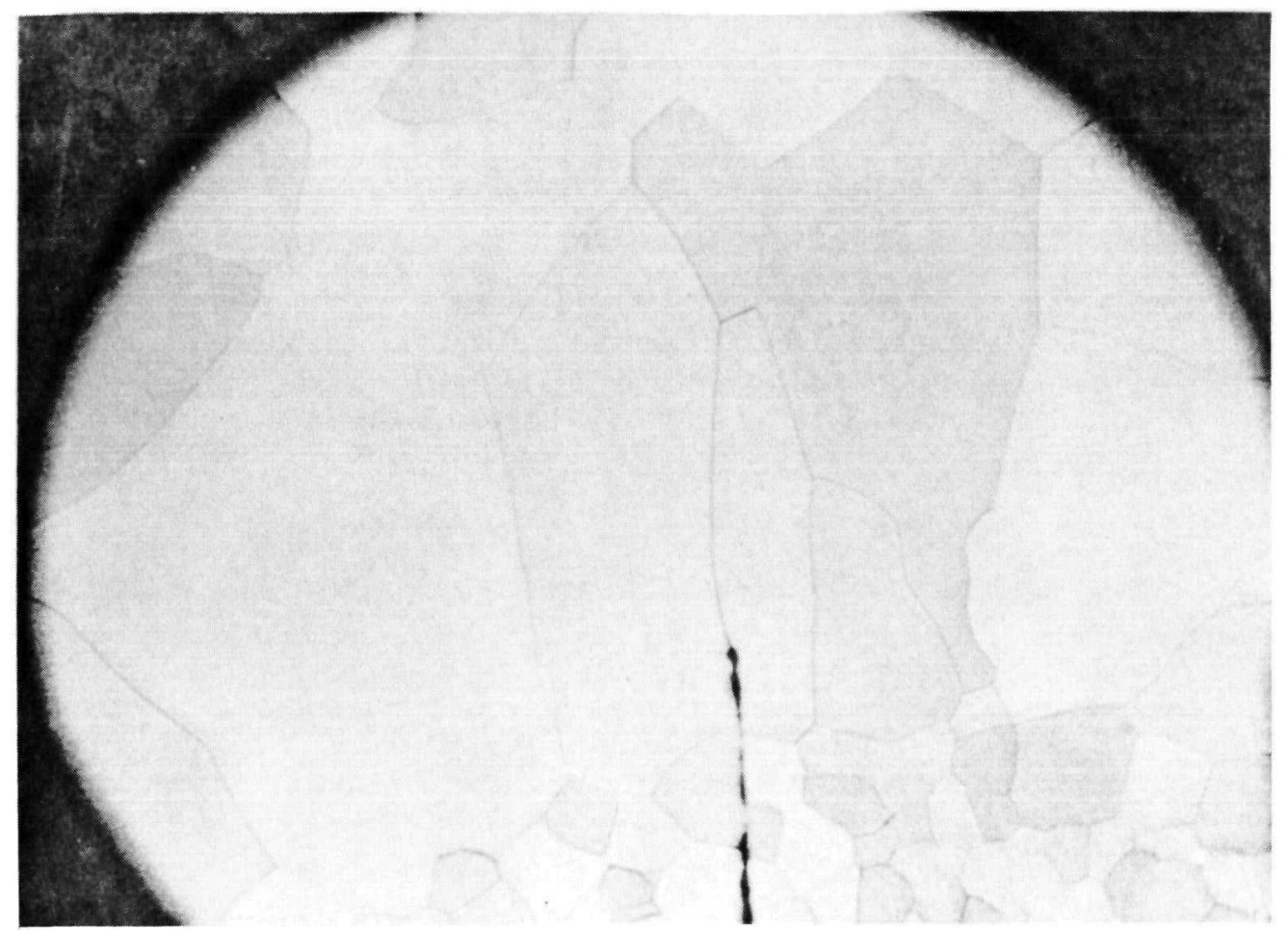

Fig. 46. Weld Zone of Molybdenum Disc R-7, Welded at 90 amps in 150 psi He--Magn 75X 
of other materials such as stainless stecl, Haynos 25 alloy, etc., have also encountered this sooting. Welding these materials with argon is a known solution to the sooting problem. Therefore, argon gas was tried in the welder, and this eliminated the sooting and produced a satisfactory weld.

The work with argon changed the heat removal rates and, therefore, the temperature of the molybdenum capsules which were being welded. This produced more control of the grain size, weld penetration and the heat-affected zone. This fact, coupled with the results obtained by varying the electrode speed, produces a very satisfactory weld. Figures 47 and 48 show the results of welding at speeds of 15 and 30 seconds per revolution, respectively.

To duplicate the ORNL operation of loading the molybdenum capsule with a heat-producing radioisotope, an electric heater was inserted in the capsule, and the capsule was welded while the capsule heater was producing power equivalent to the Cerium-144 fuel pellets. This resulted in some important changes. The temperature of the molybdenum capsule and chill blocks was much higher (approximately $400^{\circ} \mathrm{F}$ ). The chill blocks were removed and another weld was made. The heat dissipation without the chill blocks was better than with the chill blocks installed. The temperatures of the electrode holder and the gasket were satisfactorily low. This test showed that it would be possible to use this smaller welder to make the final capsule weld at ORNL.

The welds made at ORNL on molybdenum washers and fuel capsules differed somewhat from those made at Martin. A weld metal overhang invariably resulted with each weld. (See Fig. 49). It was later found that the reason for this overhang was low welding voltage. At Martin, the welding voltage was 18 volts dc. The power supply at ORNL would only go up to 12 volts, dc. It became apparent that the electrode positioning was an important factor in controlling the overhang. The problem of overhang due to the use of a 12-volt power supply was solved by adjusting the electrode positioning. Figure 50 shows the improvement in the weld.

Some molybdenum parts were irradiated in a high energy gamma field at ORNL for a 24-hour period. Some discoloration occurred from the temperature involved during the irradiation. However, these pieces exhibited no unusual effects when they were subsequently werded. 


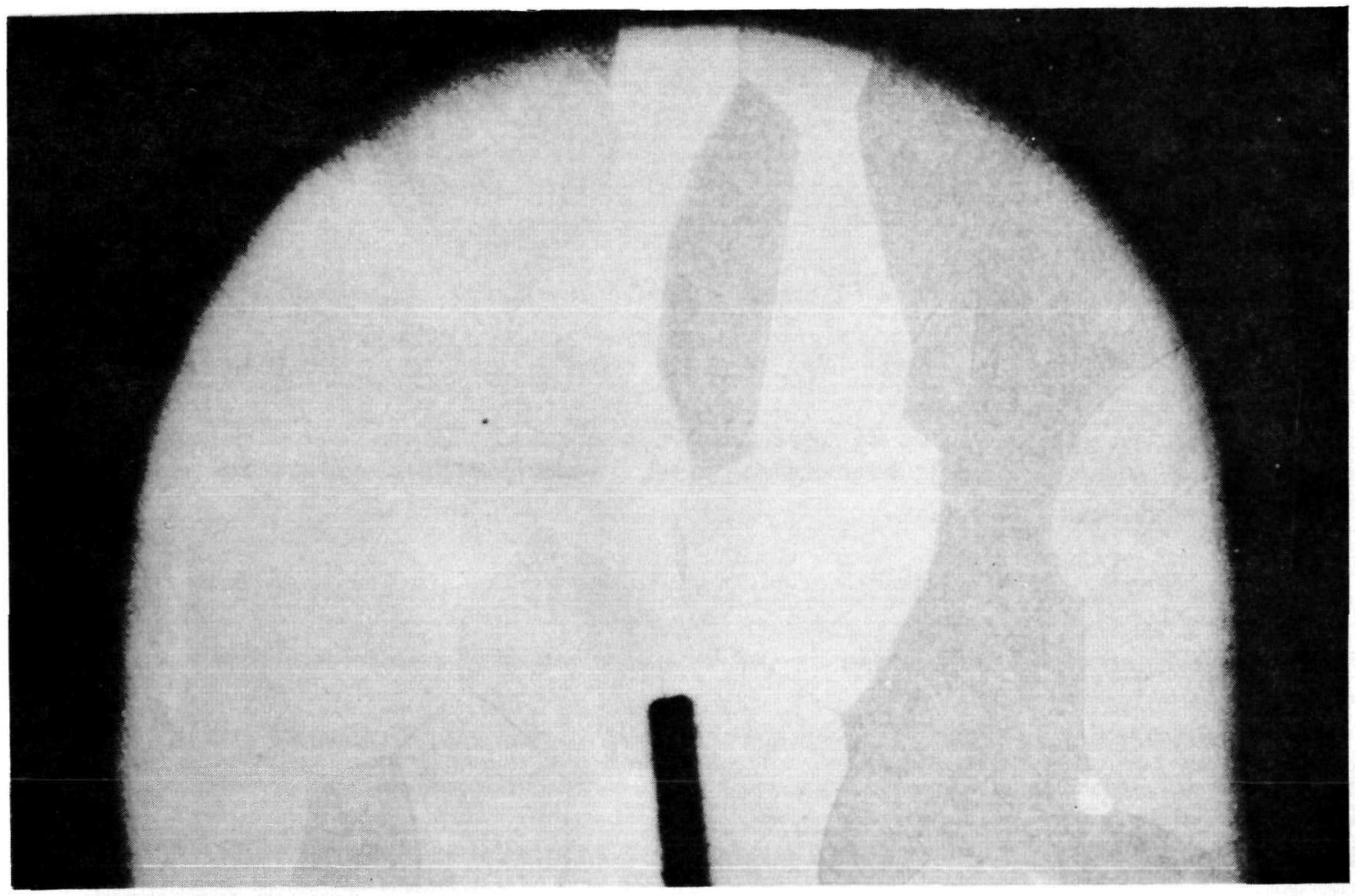

Fig. 47. Molybdenum Welded in Argon Atmosphere at 15 Seconds per Revolution--Magn 75X

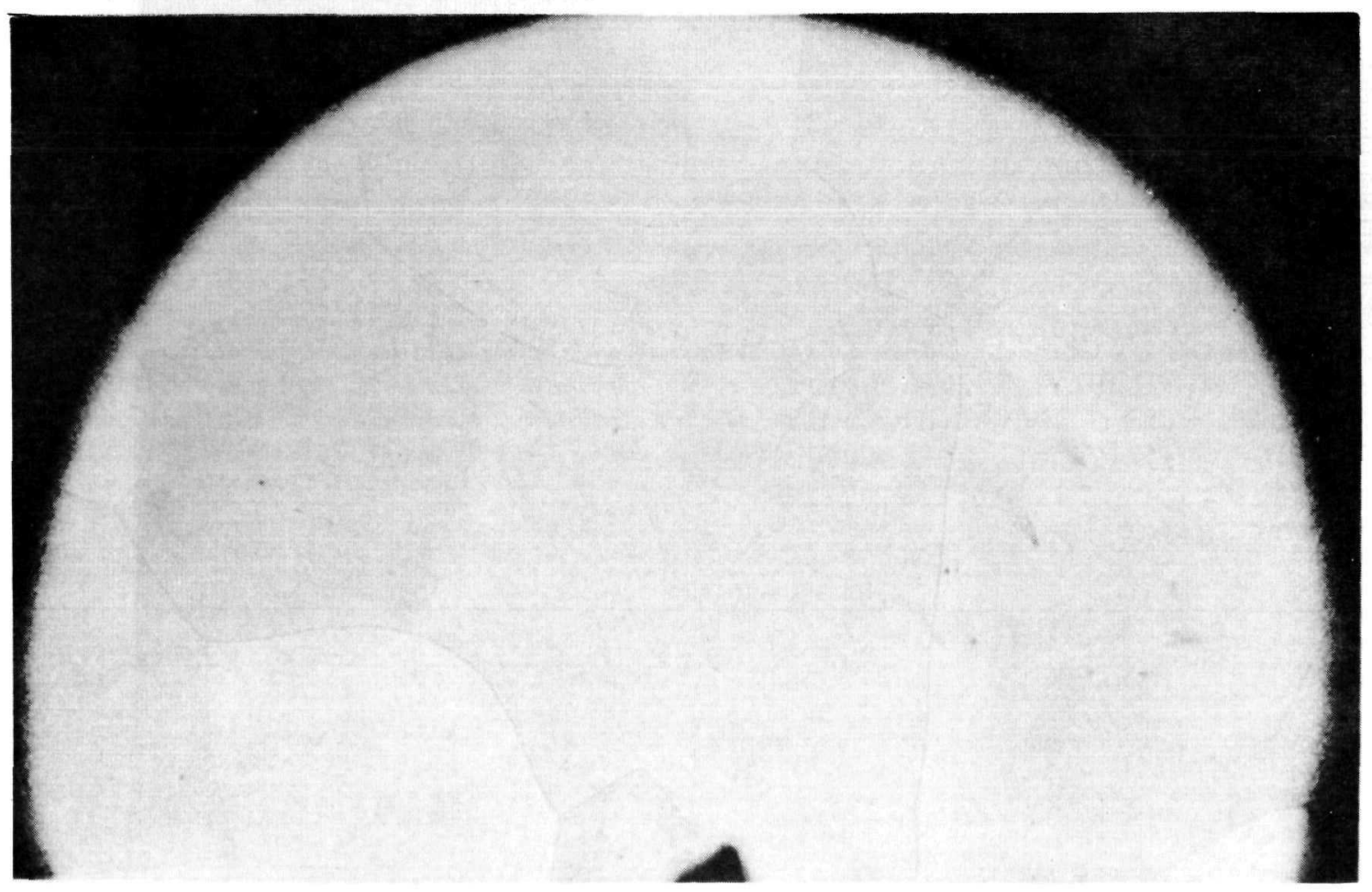

Fig. 48. Molybdenum Welded in Argon Atmosphere at 30 Seconds per Revolution--Magn 75X 


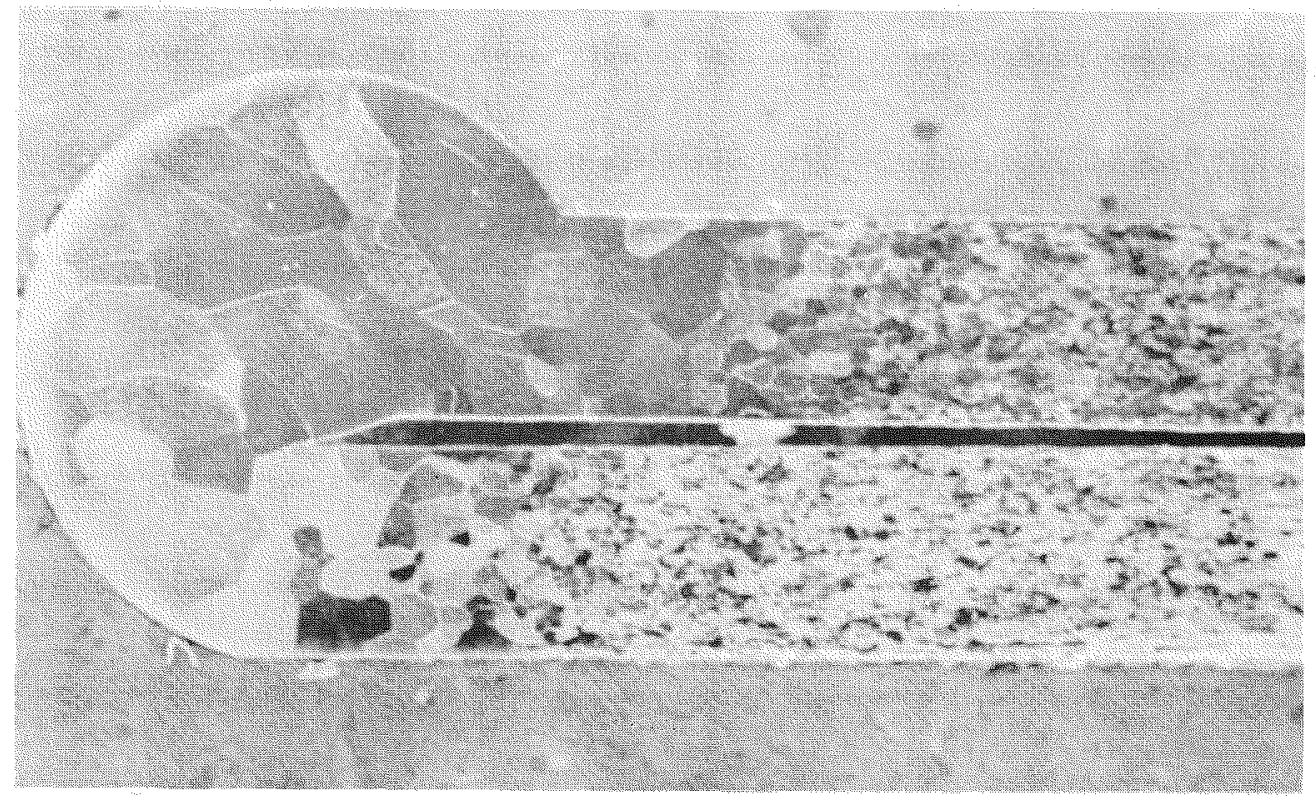

Fig. 49. 0.030-Inch Thick Molybdenum Washers Welded at ORNL in 30-psi Argon at 90 amps, Electrode Position Low--Magn 30X

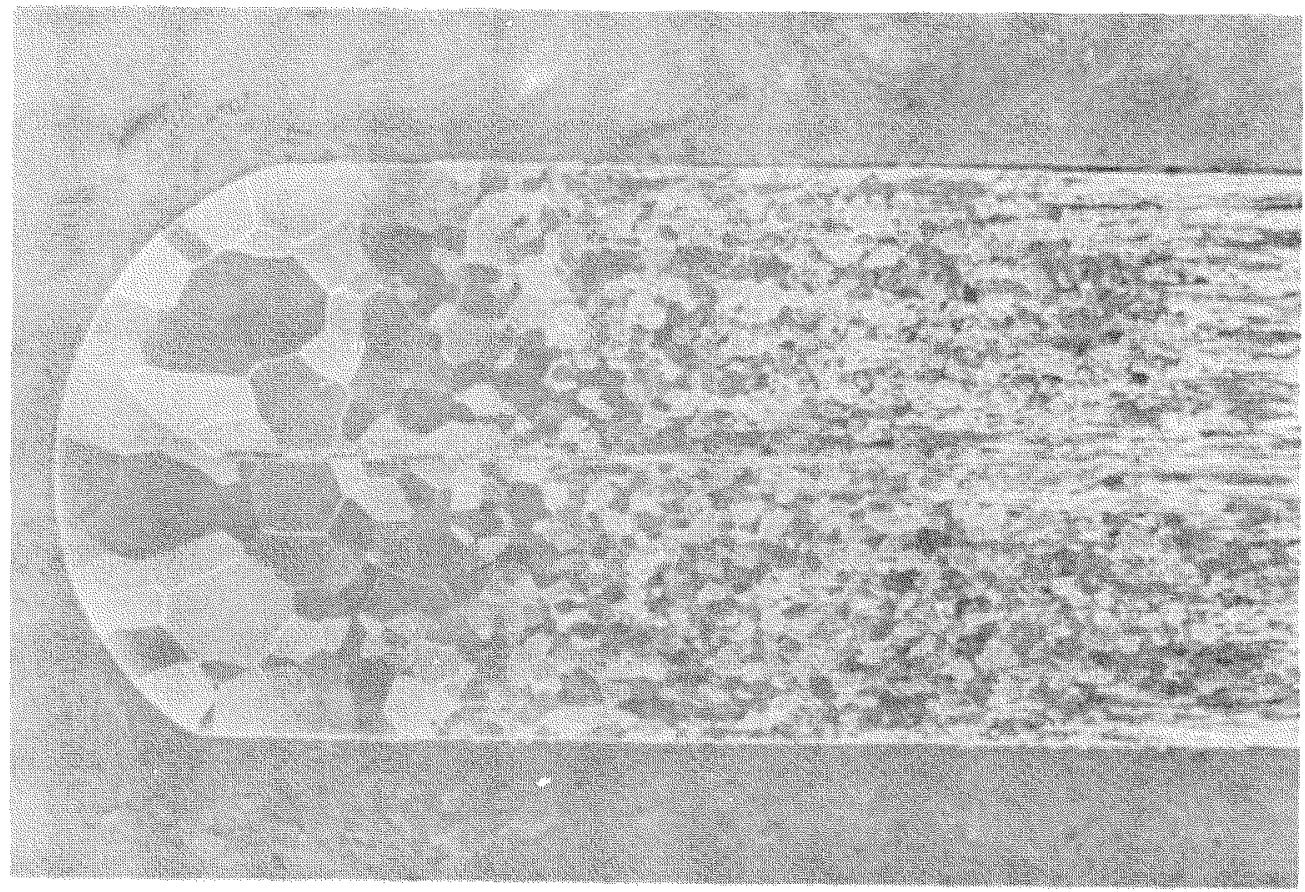

Fig. 50. 0.030-Inch Thick Molybdenum Washers Welded at ORNL in 30-psi Argon at 70 amps, Electrode Position Center-Magn 30X 


\section{Hazards Report on Ce-144 Fueled Thermionic Unit*}

A Radiological Hazards Report for the Cerium-144-fueled thermionic unit was prepared and submitted to the AEC (MND-P-2182). The report contains the following: a description of the thermionic generator, shielding container and radiocerium fuel; the results of the shielding calculations for the bare unshielded generator as well as for the shielded generator; the results of the containment analysis for mechanical integrity, thermal integrity, and chemical integrity; and the radiation hazards analysis for an atmospheric release and a release in a room.

\section{Fabrication of Containment Cask for the Ce-144-Fueled Unit}

A subcontract was let to the O.G. Kelley Company to design and fabricate a suitable containment and shipping cask for the ceriumfueled unit. The design was approved by the Bureau of Explosives and met the requirements of the ICC. The cask was fabricated and delivered to ORNL prior to November 15, 1959. $\frac{\text { 5. Fueling of the Molybdenum Fuel Capsule with the Ce-144 Fuel }}{\text { Pellets Fabricated by ORN } * *}$

A coordination meeting was held at ORNL on October 13,1959. The results of this meeting were reported in a memorandum dated October 21,1959 , which was distributed to all interested parties. The memorandum described the fueling procedure and revealed a few of the difficulties to be expected in remotely fueling the thermionic generator in a hot cell.

Early in November, personnel from ORNL visited The Martin Company to discuss the fueling of the thermionic generator with a Cerium-144 heat source. They proposed that two hot cells be used in the operation so that any contamination that may occur in the process of loading the fuel pellets into the molybdenum capsule would not be transferred to the thermionic unit. In line with this reasoning, the use of two welders, one to weld the molybdenum fuel capsule and one to weld the monel shell of the thermionic generator would greatly simplify the fueling operation. Immediate plans were made to use Martin's small welder tor the molydaenum capsuie and iile newiy developed large welder for the monel shell of the thermionic generator.

$* \mathrm{G}$. Dix
$* *$ W. Bierds 
The ORNL representatives did not particularly care for the idea of using the mass spectrometer-type leak detector. They felt that it unduly complicated the hot cell operation. As an alternate, they proposed the use of a Krypton-85 leak detector system which they had developed. In this system, a small amount of radioactive Krypton -85 gas is added to the argon atmosphere in the welder. When the capsule is welded, a certain quantity of the Krypton is trapped in the capsule. The fueled capsule is then placed in a container, the container evacuated and the leak rate determined by radioactive counting methods. The Martin Company agreed to use this method of leak detection.

After the decision was made not to fuel the thermionic unit, consideration was given to what data could be derived from the fueled molybdenum capsule.

During an ORNL visit, several discussions were held with the ORNL staff relative to the tests that would be conducted on the Cerium-144fueled capsule. The following items were tentatively agreed upon.

(1) Martin will make a device for measuring the thermal expansion of the radioactive ceric oxide pellets. This will consist of a dial indicator tool employing comparator techniques. The ambient conditions could be altered and the temperature and the length of the ceric oxide pellet would be observed.

(2) Martin will expose a molybdenum capsule containing an inert ceric oxide pellet to a measured amount of oxygen, while maintaining a wall temperature of $2300^{\circ} \mathrm{F}_{\text {。 }}$. The formation of an oxidized surface on the molybdenum and the fusing of the ceric oxide to this surface will be studied. Martin will also work with a thermocouple for measuring container temperature that could later be used in hot cell studies.

(3) A device will be made by Martin to take a sample of the gas generated within the molybdenum capsule after a half life has elapsed. The present thinking is toward a unit that would seize a tube and open the sealed end of the tube. The gas sample could be taken by means of a side arm leading to a vacuum bottle.

(4) ORNL would measure the attenuation of the gamma radiation due to the molybdenum fuel capsule.

(5) The containment vessel to hold the molybdenum capsule for storage during its half life would be designed by ORNL. It would have sufficient insulation and vacuum to achieve an 
initial molybdenum surface temperature of $2100^{\circ} \mathrm{F}$ to $2300^{\circ} \mathrm{F}$. The thermocouple well to measure this temperature will be part of the design. This unit would be fabricated by ORNL.

(6) Equipment and techniques for cutting open the molybdenum container in a hot cell after the half life will be developed by ORNL。 Metallographic studies will be made of the molybdenum, the weld area and, in particular, the ceric oxide-to-molybdenum interfaces. 


\section{SUBTASK 5.5--OPERA TIONAL THERMOELECTRIC 2- TO 5-WATT GENERATOR FOR SPACE USE*}

The objectives of this subtask are to prove the operational capabilities of the SNAP III type generator through tests simulating the anticipated environments in space and to develop conceptual designs of the SNAP III type generator to produce 2 to 5 watts of electrical power. The program consists of two phases: generator operational tests and system development. Although these phases are being conducted concurrently, the test results will be used to evaluate the system conceptual designs. The details of this program are available in the Statement of Work, MND-P-2199, and the Program Plan, MND-P-2175.

\section{A, GENERATOR OPERATIONAL TESTS}

The generator test program is subdivided according to the environments existing during the operational or missile failure and re-entry phases of the mission.

\section{Operational Environments}

The environments the generator is subjected to during this phase may be classified by origin as vehicle- or ambient-induced.

a. Vehicle-induced

The acceleration, vibration, and shock tests of the $3 \mathrm{M}-1 \mathrm{G} 4,-1 \mathrm{G} 5$, $-1 \mathrm{H} 2$ and $-1 \mathrm{H} 3$ electrically heated generators were completed with satisfactory performance throughout the program. A three-volume topical report, MND-P-2101, describes the tests in detail.

b. Ambient-induced

These tests will determine the performance of the existing generator when subjected to the temperatures and pressures anticipated at orbital altitudes. Additional tests are being conducted to develop a reliable method of power flattening and to determine the effectiveness of coatings applied to the thermoelectric elements and the generator external surface.

Limited tests of various generators were conducted in an evacuated bell jar to obtain performance comparisons. The difference in output was found to be insignificant, although a slight deterioration was noted as hours of operation increased.

*R. Wilson 
The first two phases of the altitude chamber tests on the $3 \mathrm{M}-1 \mathrm{G} 4$ unit with a bare surface and the same unit with a painted surface have been completed with satisfactory results. Voltage versus amperage data were plotted with input wattage as a parameter for ambient temperatures of $+70,+50,0,-40$ and $-100^{\circ} \mathrm{F}$. The generator was suspended inside a sealed spherical shell 24 inches in diameter. The shell internal surface was coated with a flat black lacquer to simulate the absorptivity of the space void. The shell was evacuated while chamber pressure was maintained at one atmosphere to provide a constant shell temperature. This installation is illustrated in Figs. 51 and 52. Figure 53 is a typical plot of generator performance at $-100^{\circ} \mathrm{F}$ ambient temperature with a bare external surface.

The same unit was painted with two coats of black lacquer and reinstalled; its performance is plotted in Fig. 54. The maximum generator outputs for each ambient temperature consistent with hot and cold junction temperature limitations are shown in Figs. 55 and 56. The next phase of the chamber tests will determine generator performance with a black copper oxide coating on the external surface.

The controlled gas leak method of power flattening was proven to be feasible for periods up to $70 \%$ of the radioisotope half life by preliminary proof-of-principle tests. This test work is summarized in Report MND$\mathrm{P}-2167$.

Contrary to expectations, procurement of a commercially fabricated orifice for additional testing was difficult since vendors were reluctant to guarantee the desired leak rate tolerance and flow rate as a function of pressure. In the interim, a variable orifice was designed and fabricated but calibration tests were discontinued when an adequate valve seat material could not be developed without additional testing. Several orifices fabricated by rolling copper tubing possessed a pressure exponent of 1.22 as compared to the desired exponent of 1.0. This method of fabrication is not recommended since desired characteristics can only be developed through a series of time-consuming calibrations during the fabrication process.

The commercially fabricated orifice was received late in the period. Its leak rate was $2-1 / 2 \%$ lower than desired and the pressure flow rate was nonlinear, with a pressure exponent of 1.46 . The combination of these deviations from the optimum would result in an internal generator pres sure $135 \mathrm{~mm}$ of $\mathrm{Hg}$ higher than desired after 32 days of operation. The resultant deviation in electrical power output would be small, since the power dumped would be only 3-1/2 thermal watts less than optimum at this time. A life test of the generator and orifice combination was initiated using less than one atmosphere of internal pressure to compensate for the lower leak rate of this orifice. 


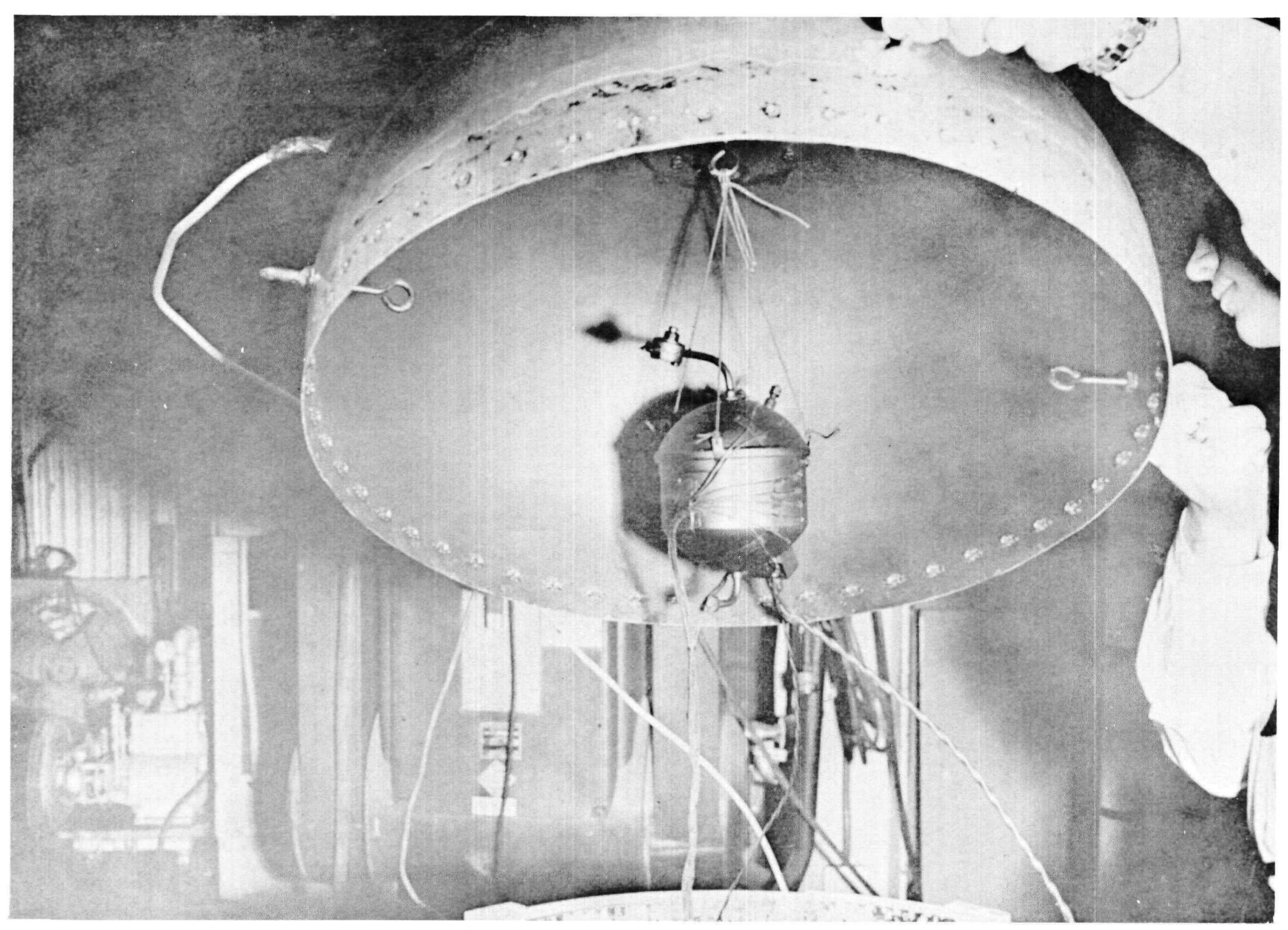

Fig. 51. Generator Installation for Altitude Chamber Tests 


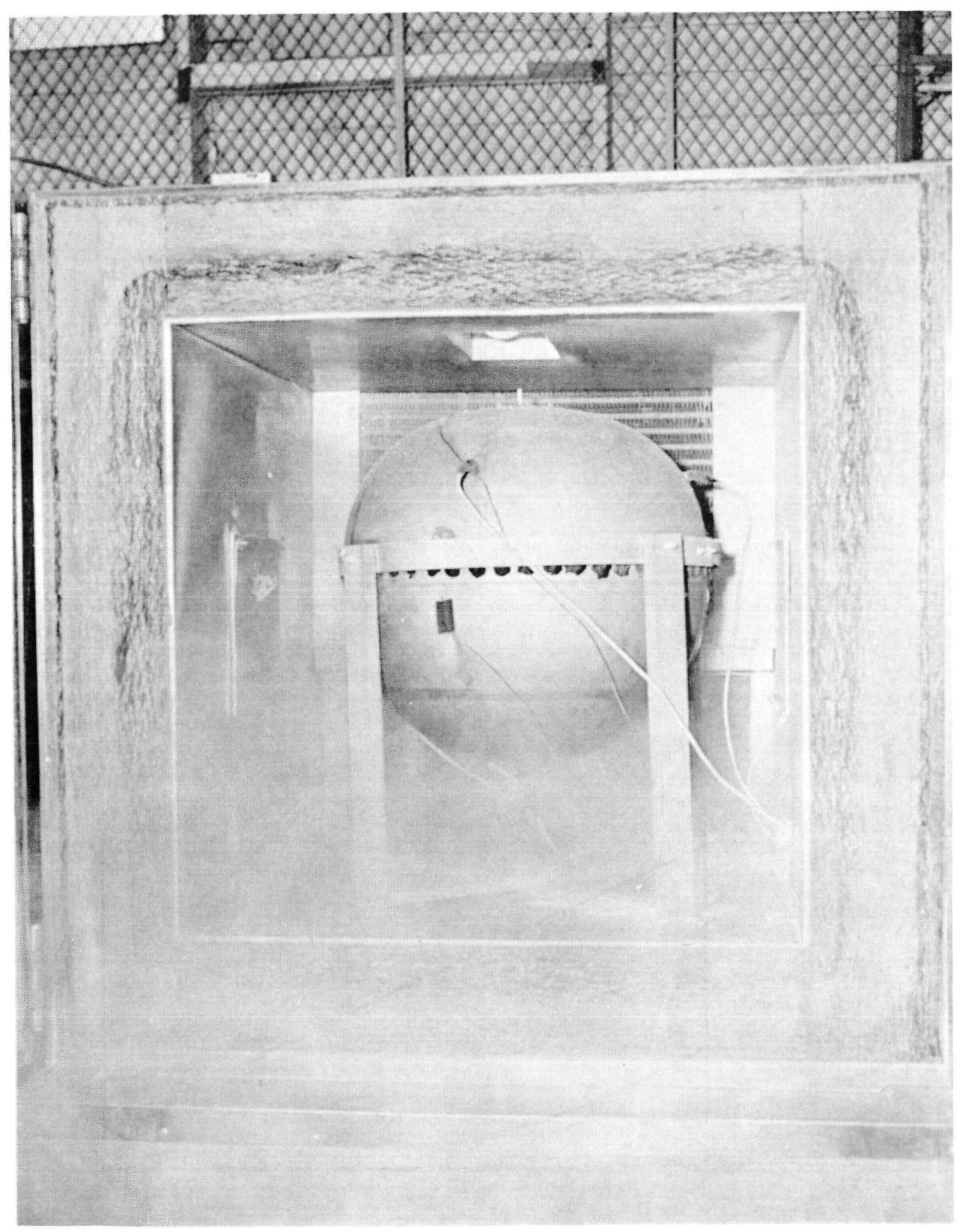

Fig. 52. Generator Installation in Altitude Chamber 


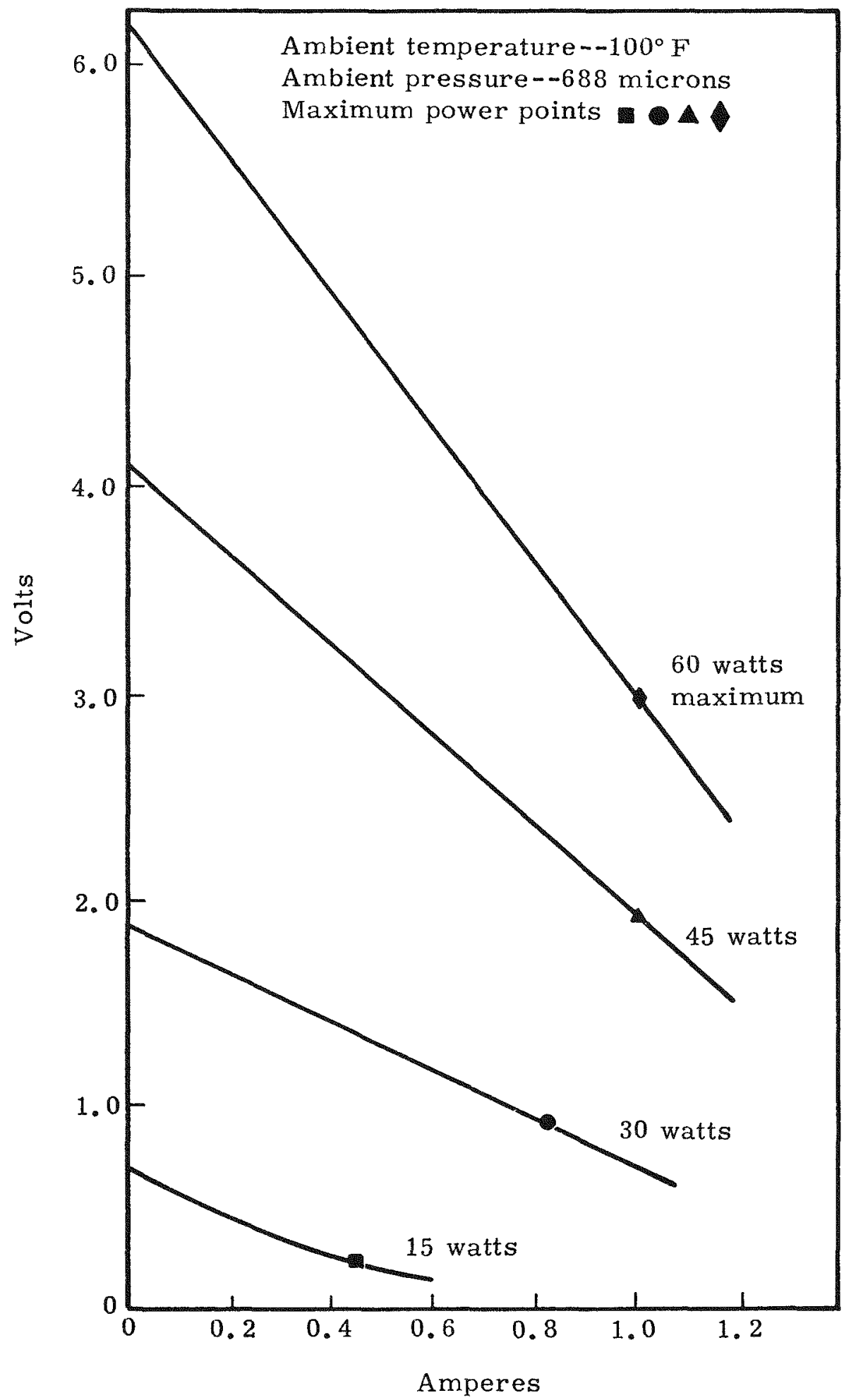

Fig. 53. Altitude Chamber Test Results, Bare Generator Surface 


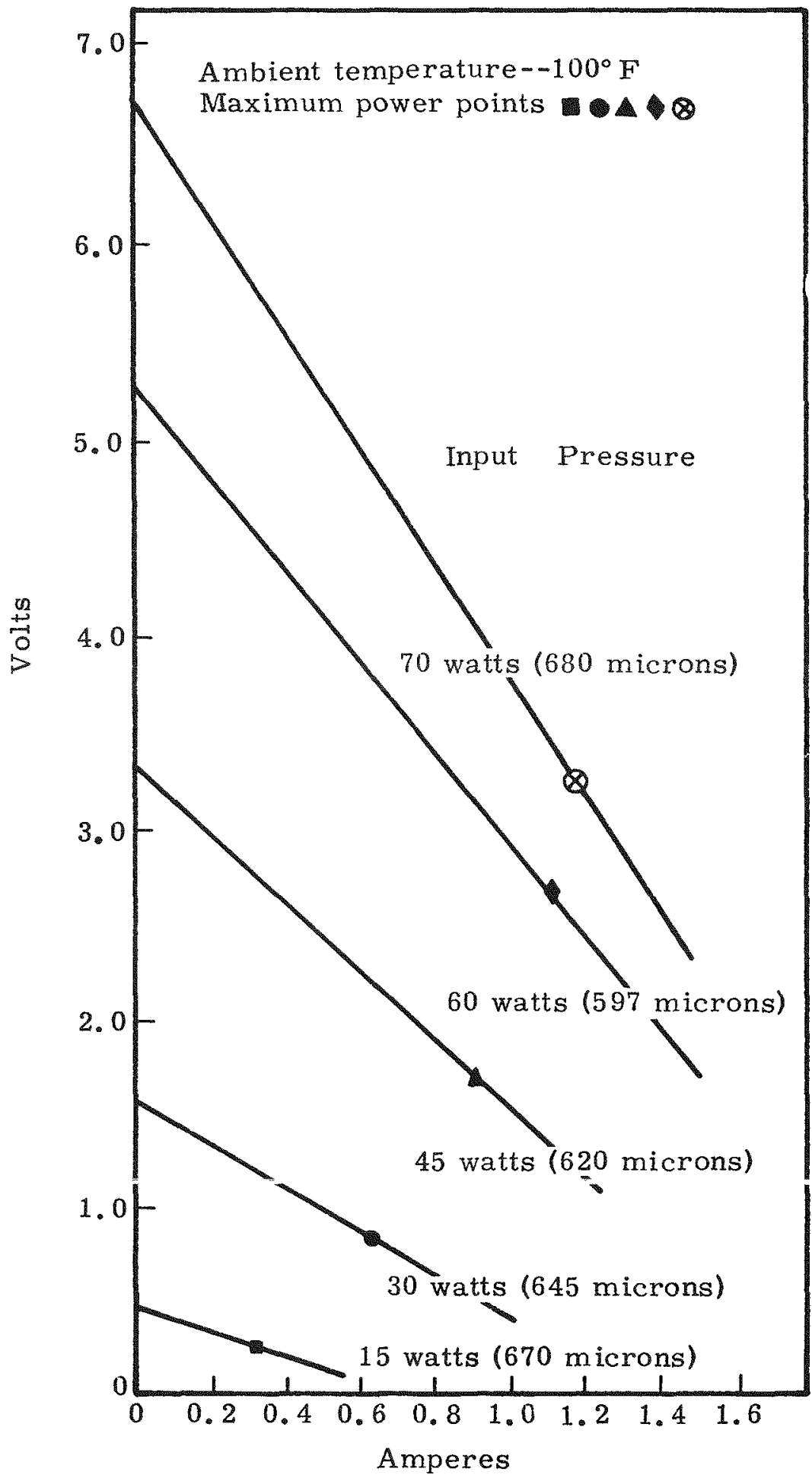

Fig. 54. Altitude Chamber Test Results, Painted Generator Surface 


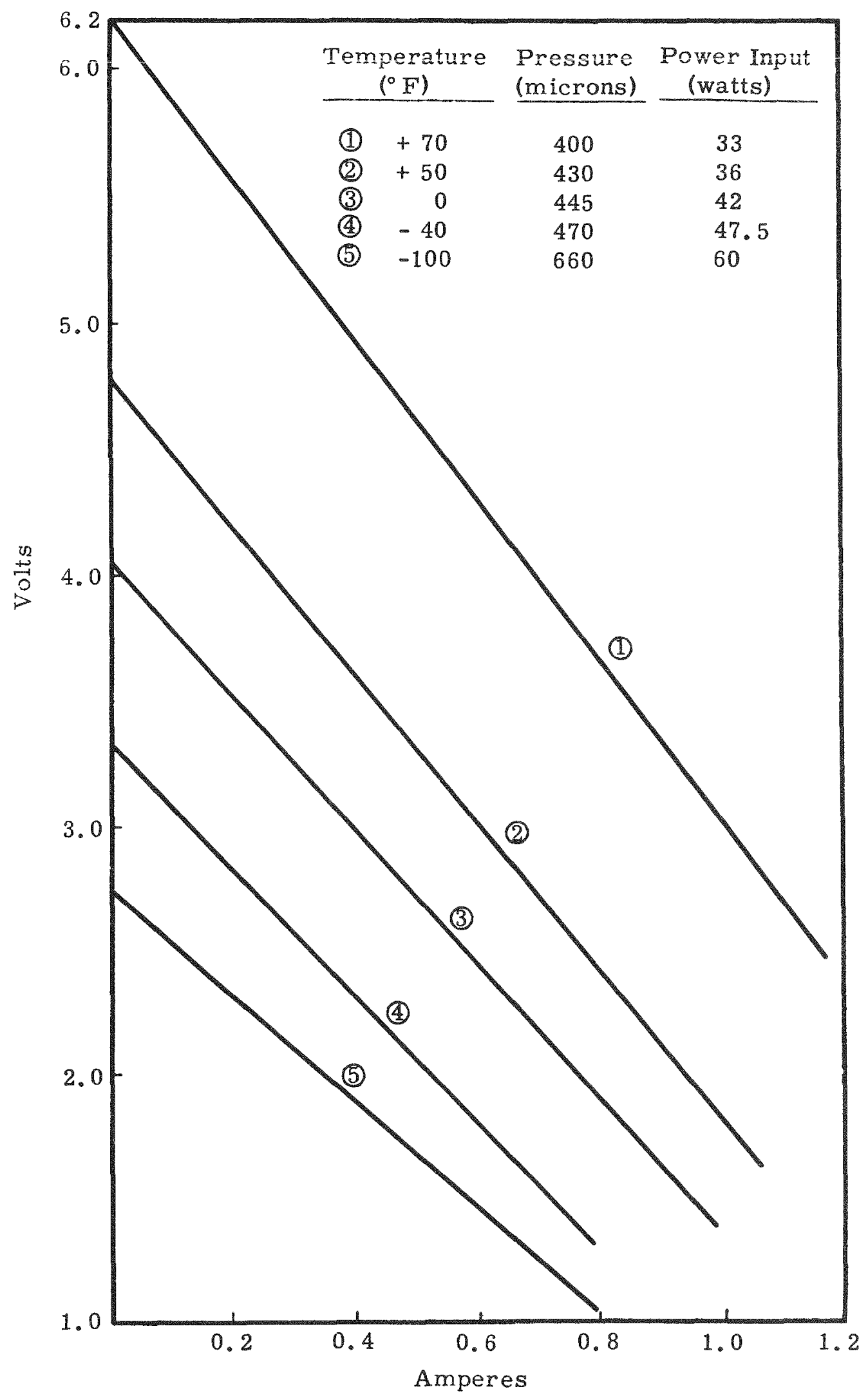

Fig. 55. Altitude Chamber Test Summary, Bare Generator Surface 


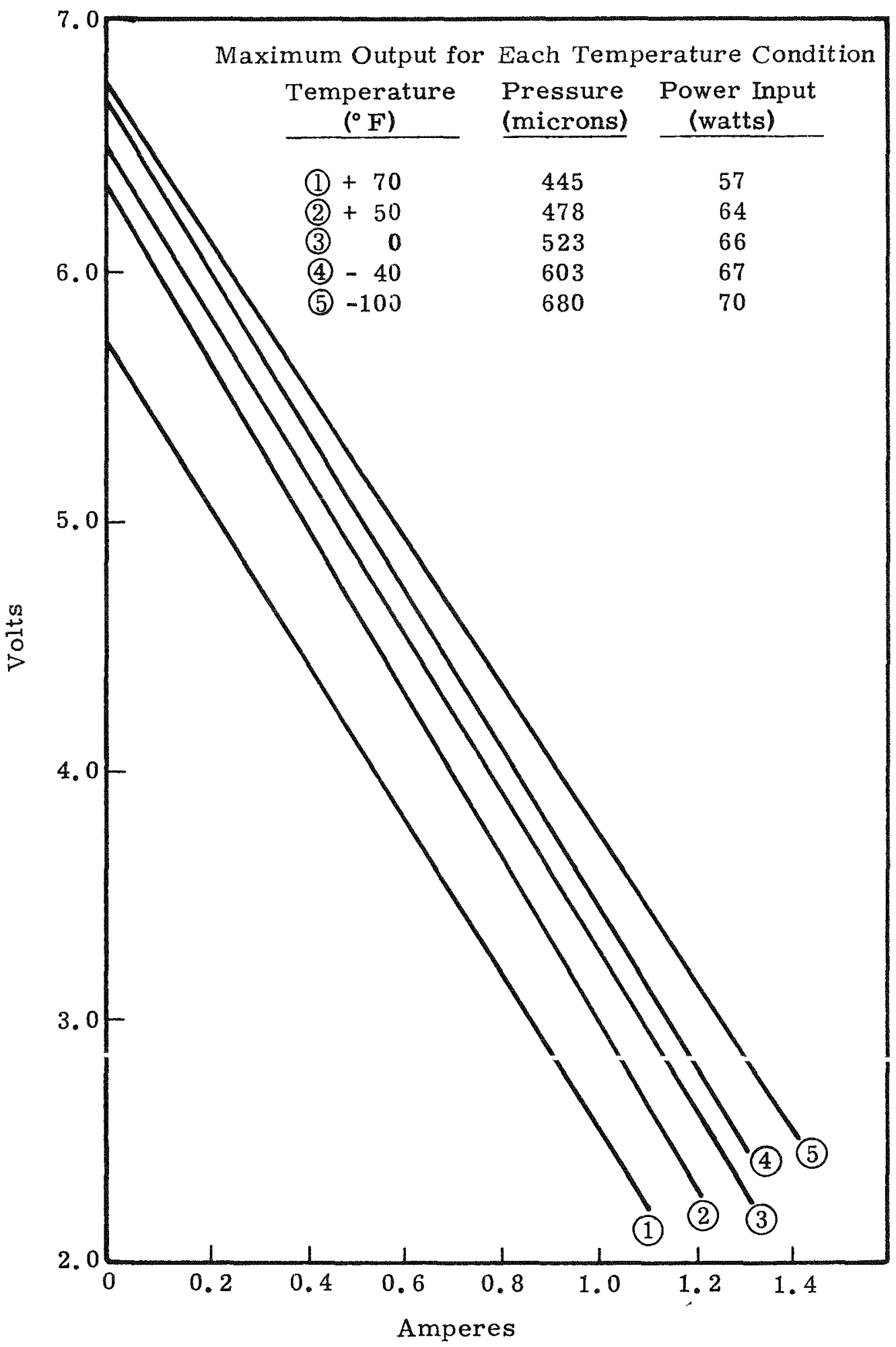

Fig. 56. Altitude Chamber Test Summary, Painted Generator Surface 
Tests to evaluate the effectiveness of various flame-sprayed coatings in reducing the sublimation rate of lead telluride thermoelectric elements were continued. Single coatings of aluminum or zirconium oxide reduced the sublimation rate significantly, but reliability is reduced by flaws in the coating. These flaws develop as a result of differential expansions during the heating cycle.

To investigate this differential expansion effect, coatings were sprayed on copper, which was selected for its high thermal expansion rate. When the specimens were heated at $1100^{\circ} \mathrm{F}$ for 36 hours, all single oxide coatings developed severe cracks. Specimens with a second metal coat applied over the oxide displayed good adherence with no visible cracking. Numerous second coat materials were then furnace tested on actual lead telluride elements. On the basis of these tests and because it has little effect on the electrical properties of lead telluride, flame-sprayed iron was selected as a second coat material. Data from the furnace tests are listed in Table 5. The results indicate a slight weight gain in some cases due to oxidation of the metal coat. Oxidation would be reduced in the actual generator installation by purging with reducing gas prior to generator operation.

Another method of reducing sublimation has been investigated and offers promise. This method utilizes a sealed ceramic sleeve to both protect and support the thermoelectric element. The assembly consists of an iron base plate to which a thin-walled short stainless steel cylinder is attached using silver solder. An aluminum oxide ceramic sleeve is inserted into the stainless steel cylinder. A compatible expansion effect was achieved in that the thin-walled stainless steel cylinder exerted sufficient force to effect a seal without cracking the ceramic. Development work will be continued and, if this method shows sufficient promise, it will be included in the electrically heated fixture tests.

A number of organic and inorganic materials with properties superior to lacquer were evaluated as external generator surface coatings. The evaluations resulted in the following recommended coating procedures for the generator case materials.

Stainless steel. Immersion in molten sodium dichromate at a temperature of $740^{\circ} \mathrm{F}$ produces a dull black oxide coating. This coating proved adequate with respect to heat stability, mercury corrosion resistance and adherence.

Copper. Immersion in two patented Du-Lite Chemical solutions at a temperature of $240^{\circ} \mathrm{F}$ produces a dull black oxide coating. This coating demonstrated good heat stability and adherence but poor mercury and acid corrosion resistance. 
TABLE 5

Weight Loss of Coated PbTe Elements

(Furnace pressure--20 microns)

\begin{tabular}{|c|c|c|c|c|c|}
\hline Sample & $\begin{array}{c}\text { Time of } \\
\text { Test } \\
\text { (hr) }\end{array}$ & $\begin{array}{c}\text { Temperature } \\
\left({ }^{\circ} \mathrm{F}\right)\end{array}$ & $\begin{array}{l}\text { Weight Loss } \\
\text { (gm) }\end{array}$ & $\begin{array}{c}\text { Weight Loss } \\
(\%)\end{array}$ & $\begin{array}{c}\text { Weight Loss/ } \\
\text { Area/Hour } \\
\left(\mathrm{gm} / \mathrm{cm}^{2} / \mathrm{hr}\right) \times 10^{-6} \\
\end{array}$ \\
\hline $\mathrm{PbTe}$ (uncoated) & 192.5 & 900 & 0.1207 & 19.19 & 111.97 \\
\hline PbTe (uncoated) & 192.5 & 900 & 0.1312 & 20.75 & 136.95 \\
\hline $\mathrm{PbTe}-\mathrm{Al}_{2} \mathrm{O}_{3}$ & 233.0 & 900 & 0.0075 & 0.11 & 5.78 \\
\hline $\mathrm{PbTe}-\mathrm{Al}_{2} \mathrm{O}_{3}$ & 233.0 & 900 & 0.0046 & 0.07 & 3.54 \\
\hline $\mathrm{Pb}^{\prime} \mathrm{Te}-\mathrm{ZrO}_{2}$ & 233.0 & 900 & 0.0050 & 0.08 & 3.85 \\
\hline $\mathrm{PbTe}-\mathrm{ZrO}_{2}$ & 233.0 & 900 & 0.0069 & 0.10 & 5.31 \\
\hline $\mathrm{PbTe}-\mathrm{Al}_{2} \mathrm{O}_{3}$ & 400.0 & 900 & 0.0096 & 0.14 & 4.31 \\
\hline $\mathrm{PbTe}-\mathrm{Al}_{2} \mathrm{O}_{3}$ & 400.0 & 900 & 0.0067 & 0.10 & 3.01 \\
\hline $\mathrm{PbTe}-\mathrm{ZrO}_{2}$ & 400.0 & 900 & 0.0063 & 0.09 & 2.83 \\
\hline $\mathrm{PbTe}-\mathrm{ZrO}_{2}$ & 400.0 & 900 & 0.0090 & 0.14 & 4.04 \\
\hline $\mathrm{PbTe}-\mathrm{Cr}_{2} \mathrm{O}_{3}$ & 197.5 & 1000 & 0.0221 & 0.80 & 27.70 \\
\hline $\mathrm{PbTe}_{-\mathrm{ZrSiO}_{4}}$ & 197.5 & 1000 & 0.0108 & 0.50 & 23.00 \\
\hline $\mathrm{PbTe}-\mathrm{Al}_{2} \mathrm{O}_{3}$ & 191.5 & 1000 & 0.0723 & 1.18 & 81.36 \\
\hline $\mathrm{PbTe}-\mathrm{Al}_{2} \mathrm{O}_{3}$ & 191.5 & 1000 & 0.0231 & 0.33 & 21.64 \\
\hline $\mathrm{PbTe}-\mathrm{ZrO}_{2}$ & 191.5 & 1000 & 0.0030 & 0.15 & 7.12 \\
\hline $\mathrm{PbTe}-\mathrm{ZrO}_{2}$ & 191.5 & 1000 & 0.0011 & 0.06 & 2.71 \\
\hline $\mathrm{PbTe}-\mathrm{ZrO}_{2}$ & 194.5 & 1000 & 0.0052 & 0.13 & 26.30 \\
\hline $\mathrm{PbTe}-\mathrm{ZrO}_{2}-\mathrm{Ni}$ (rod) & 194.5 & 1000 & 0.0009 & 0.02 & 1.49 \\
\hline $\mathrm{PbTe}-\mathrm{ZrO}_{2}-\mathrm{Al}$ (rod) & 194.5 & 1000 & +0.0012 & +0.04 & Weight gain \\
\hline $\mathrm{PbTe}-\mathrm{ZrO}_{2}-\mathrm{SS}$ (rod) & 194.5 & 1000 & 0.0018 & 0.07 & 3.69 \\
\hline 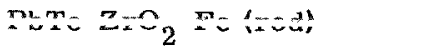 & 101.5 & $\underline{n} \Omega \Omega$ & $\pm n 002 n$ & $\pm n_{1} 16$ & Waight grin \\
\hline $\mathrm{PbTe}-\mathrm{Al}_{2} \mathrm{O}_{3}$-Electroless $\mathrm{Ni}$ & 197.5 & 1000 & 0.0016 & 0.04 & 2.41 \\
\hline $\mathrm{PbTe}-\mathrm{Al}_{2} \mathrm{O}_{3}-$ Electroless $\mathrm{Cu}$ & 197.5 & 1000 & 0.0016 & 0.04 & 2.06 \\
\hline $\mathrm{PbTe}-\mathrm{Al}_{2} \mathrm{O}_{3}-\mathrm{Al}$ (powder) & 197.5 & 1000 & 0.0085 & 0.20 & 14.50 \\
\hline $\mathrm{PbTe}-\mathrm{Al}_{2} \mathrm{O}_{3}-\mathrm{SS}$ (powder) & 197.5 & 1000 & 0.0012 & 0.02 & 1.15 \\
\hline $\mathrm{PbTe}-\mathrm{Al}_{2} \mathrm{O}_{3}-\mathrm{Fe}$ (rod) & 197.5 & 1000 & +0.0037 & +0.10 & Weight gain \\
\hline $\mathrm{PbTe}-\mathrm{Al}_{2} \mathrm{O}_{3}-\mathrm{Ni}$ (rod) & 197.5 & 1000 & +0.0007 & +0.02 & Weight gain \\
\hline $\mathrm{PbTe}-\mathrm{Al}_{2} \mathrm{O}_{3}-$ Enamel $\mathrm{A}-5$ & 197.5 & 1000 & 0.0001 & 0.00 & 0.10 \\
\hline
\end{tabular}


Aluminum. Immersion in a sulfuric acid solution anodizes the surface, producing an aluminum oxide coat. Immersion in cobalt acetate followed by sodium sulfide forms a stable black cobalt sulfide coat that is sealed by immersion in hot water.

\section{Missile Failure Environments}

These environments consist of conditions that might be encountered through a failure of the vehicle system at any time during its trajectory。 These situations range from missile explosions and fires on the launch pad to impact on the earth's surface with subsequent exposure to corrosive media after failing to achieve orbital velocity. In addition, the generator may be ejected at any time by a cartridge actuated device which will separate the unit from the immediate area of missile failure.

\section{a. Launch pad failure}

The detonation of the booster propellant will be simulated by exposing specimens to the shock wave and temperatures created by a TNT explosion. The quantity of explosive, distance to specimens, and the specimen size will be scaled down to reduce the explosive required and to duplicate the same shock overpressure. One charge of approximately 2000 pounds of TNT will be detonated with a 1/3-scale specimen array as follows: the

3 generator mockups containing 3 capsule specimens

3 bare capsule specimens.

The fire resulting from ignition of the hyperbolic propellants used in the final stage will be simulated by mixing red fuming nitric acid and aniline. The heat created will in turn ignite a mixture of typical missile structural materials. The temperatures are expected to reach a peak of $6000^{\circ} \mathrm{F}$ and decay to 1500 to $2000^{\circ} \mathrm{F}$ over a 20 -minute period. Full-scale heated specimens will be arranged around the fire area as follows:

3 generator mockups containing 3 capsule specimens

3 bare capsule specimens.

Additional details of the test procedures are available in Statements of Work, MND-2238 and MND-2239。

These tests will be conducted at the Aberdeen Proving Ground by Army personnel and monitored by Martin personnel. The statements of work and the final cost estimate were submitted to the New York Operations Office of the AEC and tests will begin when the transfer of funds has been approved. 


\section{b. Impäct}

The failure or command destruction of the vehicle after launch will cause the generator to impact on the earth's surface with various velocities. A terminal velocity of 500 feet per second for the isotope capsule and 350 feet per second for the generator assembly was assumed to be the maximum attainable in each case and was the velocity used for the tests. The specimens were accelerated to the desired velocities by a rocket sled propelled by a single 2.75-inch FFAR rocket motor. The tests were conducted on the ballistic track at Aberdeen Proving Ground under the direction of Army personnel and monitored by Martin personnel.

The configuration of the specimens and method of heating prior to impact is as follows:

\section{Specimen}

Type

5

6

\author{
Shape \\ Cylinder with \\ hemispherical \\ end caps
}

Truncated cone

Material
$\begin{aligned} & \text { Shell-copper } \\ & \text { Capsule- } \\ & \text { Haynes } 25\end{aligned}$

1
2-1/16 Furnace

The configuration of the isotope capsule test specimen assembly (Type 6) is shown in Fig. 57. The two small stainless steel welded capsules enclosed in a single stainless steel capsule is typical of the encapsulation technique used with Polonium-210 radioisotope.

The simulated generator impact specimens were assembled as shown in Figs. 58 and 59. The only component not shown in these figures is the Min-K insulation used to fill the void between the internal capsule and the outer copper case. The powdered Min-K is difficult to work with since it is light and fluffy with poor packing qualities. Respirators and a hood are required to prevent inhalation of the airborne glass particles which are very irritating to exposed mucous membranes. Attempts to machine or form solid pieces ot insulation were siow, and airivurue par iivies wite also created. Moistening the powder with water solved the packing and airborne particle problems. After assembly, the specimens were furnaceheated to evaporate the water. Some shrinkage occurred but the amount was not significant for test specimen use.

Four types of targets were used during this program: granite, consolidated rock, unconsolidated rock and water. The granite targets were rectangular blocks $2 \times 2 \times 4-1 / 2$ feet. The density of this material was 


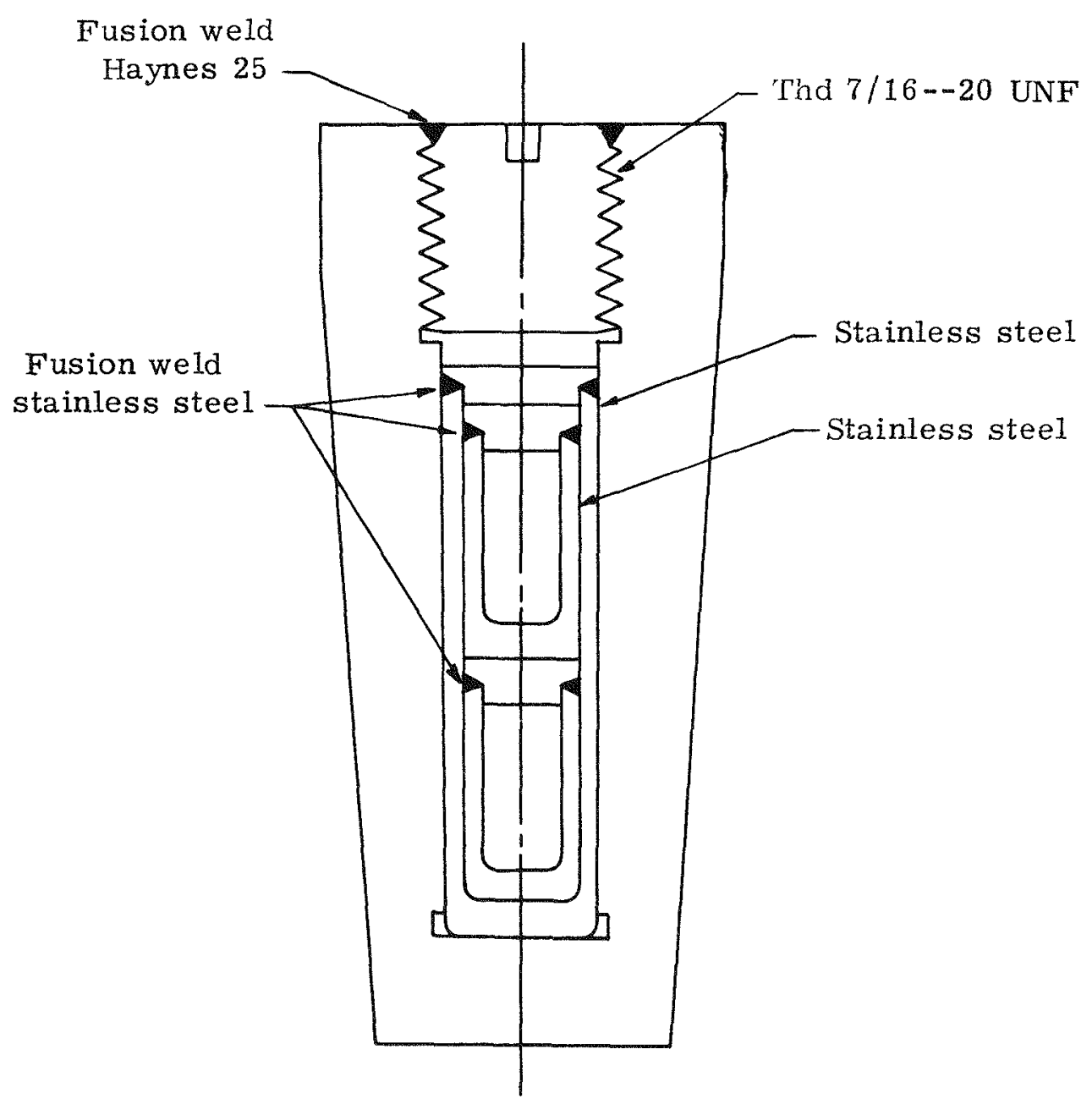

Scale: $2=1$

Fig. 57. Type 6 Test Specimen Assembly 


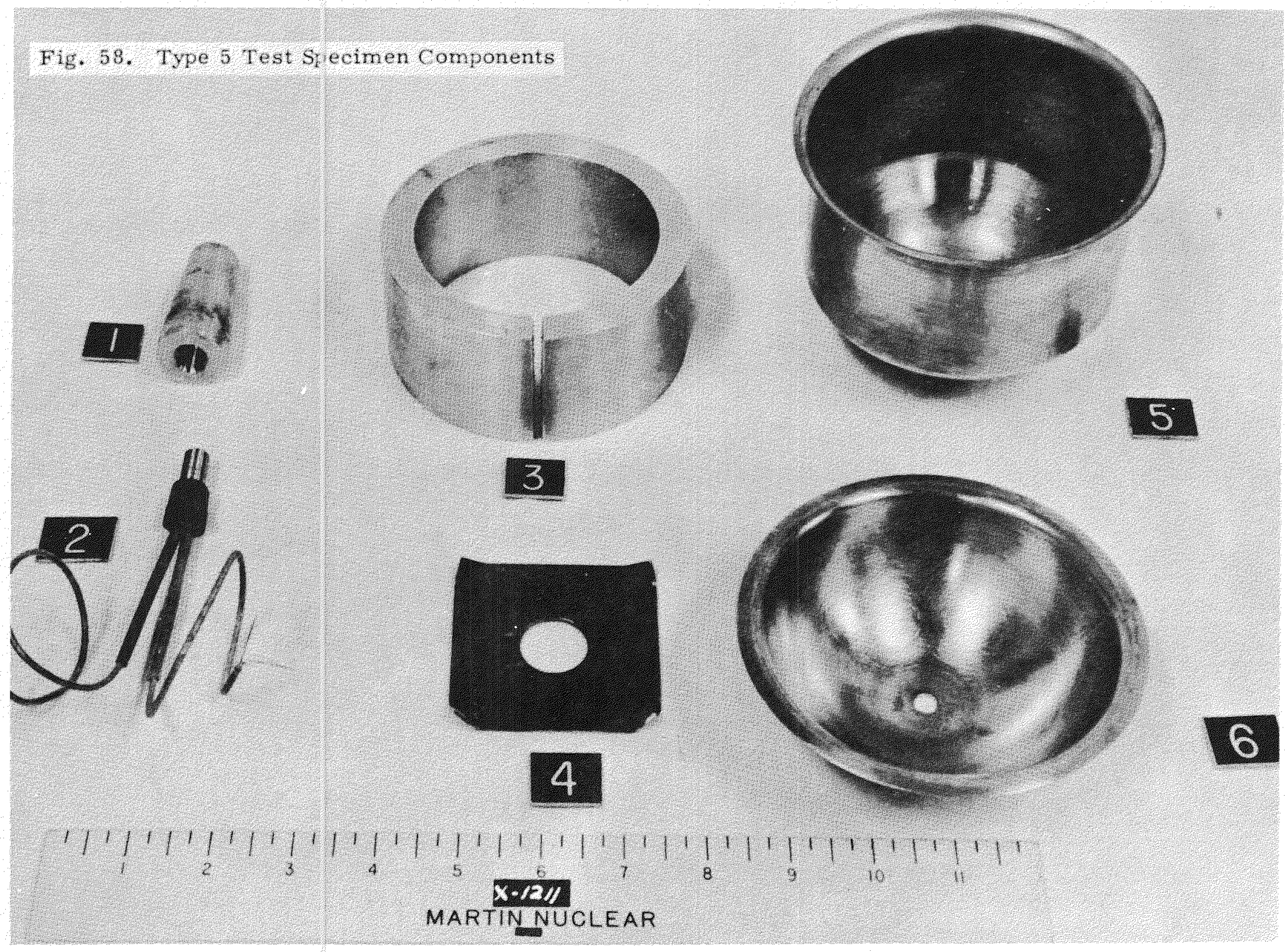




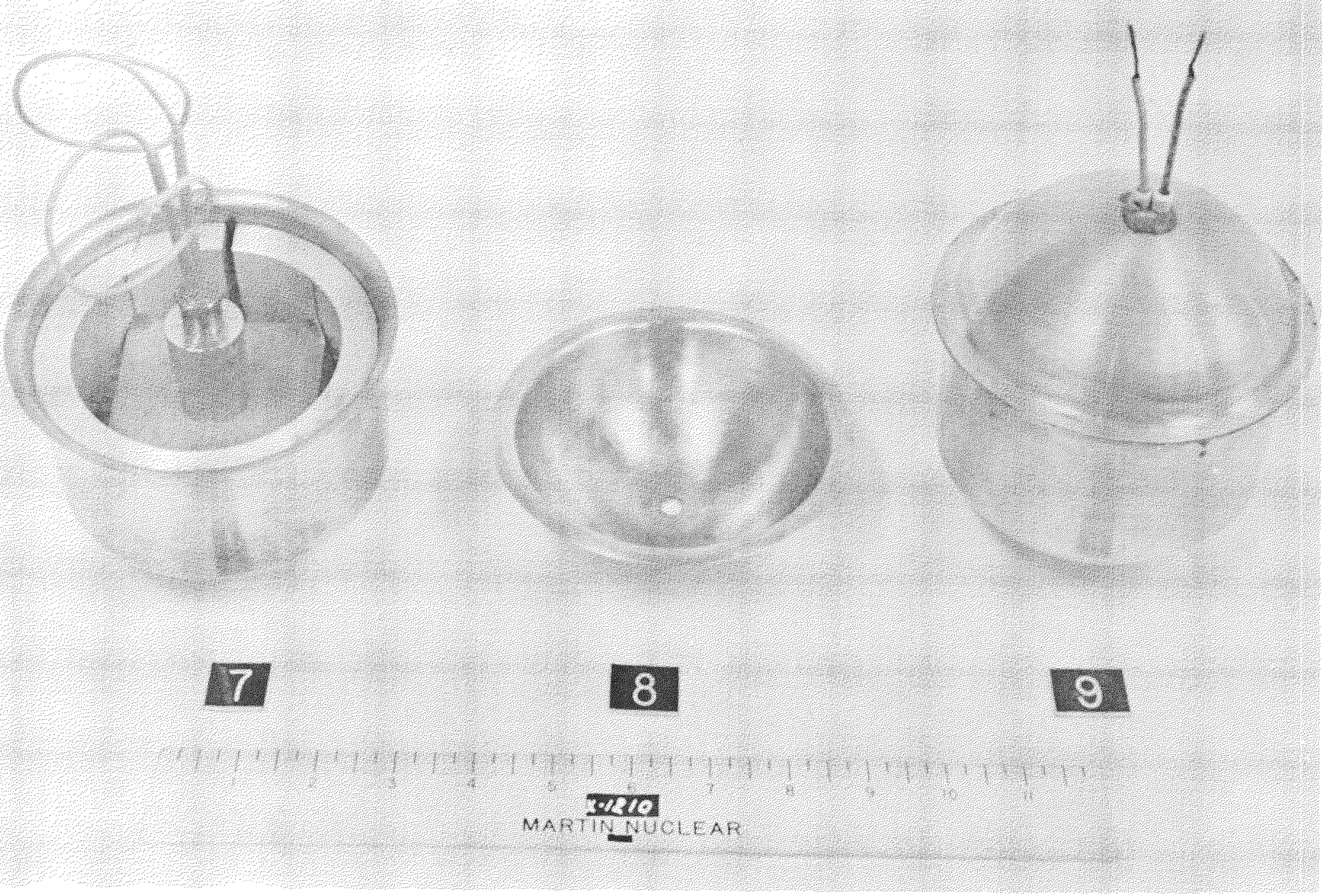

Fig. 59. Type 5 Test Specimen Assembly 
168 pounds per cubic foot, and it had an abrasive hardness similar to that of steel (Rockwell hardness C-53). The consolidated rock targets were a mixture of earth plus $20 \%$ cement and adequate water to ensure proper mixing. The targets were poured in $3 \times 3 \times 4$ foot plywood frames and allowed to cure for 26 days prior to the firing. The crushing strength of these targets was 643 pounds per square inch. The unconsolidated rock targets consisted of earth packed into a box with physical dimensions of 4 feet square by 20 feet long. The density of the earth was approximately 75 pounds per cubic foot. The water targets were portable plastic pools 10 feet in diameter by 2 feet high. The plastic liners were supported by a steel wire mesh located at the circumference of the pool. The wire was removed in the area of impact so that the specimens would contact only the plastic liner and water.

Impact test results are listed in Table 6 . Figures $60,61,62$ and 63 show the simulated generator specimens (Type 5) after impact. The only effect of the impact on the capsule was a slight deformation as a result of the impact on the granite target. The isotope capsule specimens (Type 6) were not recovered.

Aberdeen personnel attributed the failure of the specimens of Type 6 to impact the targets to unsatisfactory design of the track hardware. The details of the sled will be redesigned for this type specimen and two tests will be repeated at a future date. Two new isotope capsule specimens are being fabricated for tests during the next period. The target media for these two tests will be granite and consolidated rock. The additional track runs will be conducted at no additional cost by the Aberdeen Proving Ground because they did not meet contractual agreements.

\section{c. Corrosion}

After impact, the isotope container material will be exposed to the corrosive media existing on the earth's surface. The more serious of these in the case of a steel container are considered to be oxidizing atmosphere, salt water and salt spray.

The corrosion tests in an oxidizing atmosphere are complete. Four samples each of Haynes 25 and Type 304 stainless steel were maintained at $1100^{\circ} \mathrm{F}$ in air for a total of 136 hours. The results are as listed in Table 7 . 
TABLE 6

Impact Test Results

\begin{tabular}{|c|c|c|c|c|c|c|c|c|}
\hline $\begin{array}{c}\text { Specimen } \\
\text { Type } \\
\end{array}$ & $\begin{array}{c}\text { Specimen } \\
\text { Number }\end{array}$ & $\begin{array}{l}\text { Vehicle } \\
\text { Weight } \\
\text { (1b) }\end{array}$ & $\begin{array}{c}\text { Specimen } \\
\text { Weight } \\
\text { (gm) } \\
\end{array}$ & $\begin{array}{l}\text { Specimen } \\
\text { Tem- } \\
\text { perature } \\
(0 \mathrm{~F})\end{array}$ & $\begin{array}{c}\text { Impact } \\
\text { Velocity } \\
\text { (fps) } \\
\end{array}$ & Target & $\begin{array}{c}\text { Penetration } \\
\text { (in.) }\end{array}$ & Remarks \\
\hline 5 & 1 & 81.7 & 1590 & 925 & 318 & Granite & 0 & $\begin{array}{l}\text { Copper shell } \\
\text { ruptured--no } \\
\text { damage to core }\end{array}$ \\
\hline 5 & 2 & 82.71 & 1451 & 1032 & 335 & $\begin{array}{l}\text { Unconsolidated } \\
\text { rock }\end{array}$ & 24 & $\begin{array}{l}\text { Shell dented-- } \\
\text { no damage to } \\
\text { core }\end{array}$ \\
\hline 5 & 3 & 80.9 & 1402.5 & 1000 & 341 & Water & Note 1 & $\begin{array}{l}\text { Shell dented-- } \\
\text { no damage to } \\
\text { core }\end{array}$ \\
\hline 5 & 4 & 81.5 & 1388.5 & 850 & 342 & $\begin{array}{l}\text { Consolidated } \\
\text { rock }\end{array}$ & 9 & $\begin{array}{l}\text { Shell ruptured- } \\
\text { no damage to } \\
\text { core }\end{array}$ \\
\hline 6 & 1 & 58.8 & 210.5 & 1050 & 441 & Granite & -- & $\begin{array}{l}\text { Specimēn } \\
\text { missed target }\end{array}$ \\
\hline 6 & 2 & 59.2 & 208 & 995 & 434 & $\begin{array}{l}\text { Unconsolidated } \\
\text { rock }\end{array}$ & -- & $\begin{array}{l}\text { Specimen } \\
\text { missed target }\end{array}$ \\
\hline 6 & 3 & 59.6 & 211.25 & 1150 & 453 & Water & - & $\begin{array}{l}\text { Specimen } \\
\text { missed target }\end{array}$ \\
\hline 6 & 4 & 59.3 & 211.25 & 900 & 442 & $\begin{array}{l}\text { Consolidated } \\
\text { rock }\end{array}$ & -- & $\begin{array}{l}\text { Specimen } \\
\text { missed target }\end{array}$ \\
\hline
\end{tabular}

Note 1. The specimen traveled through the water about five feet before breaking the surface of the pool. 


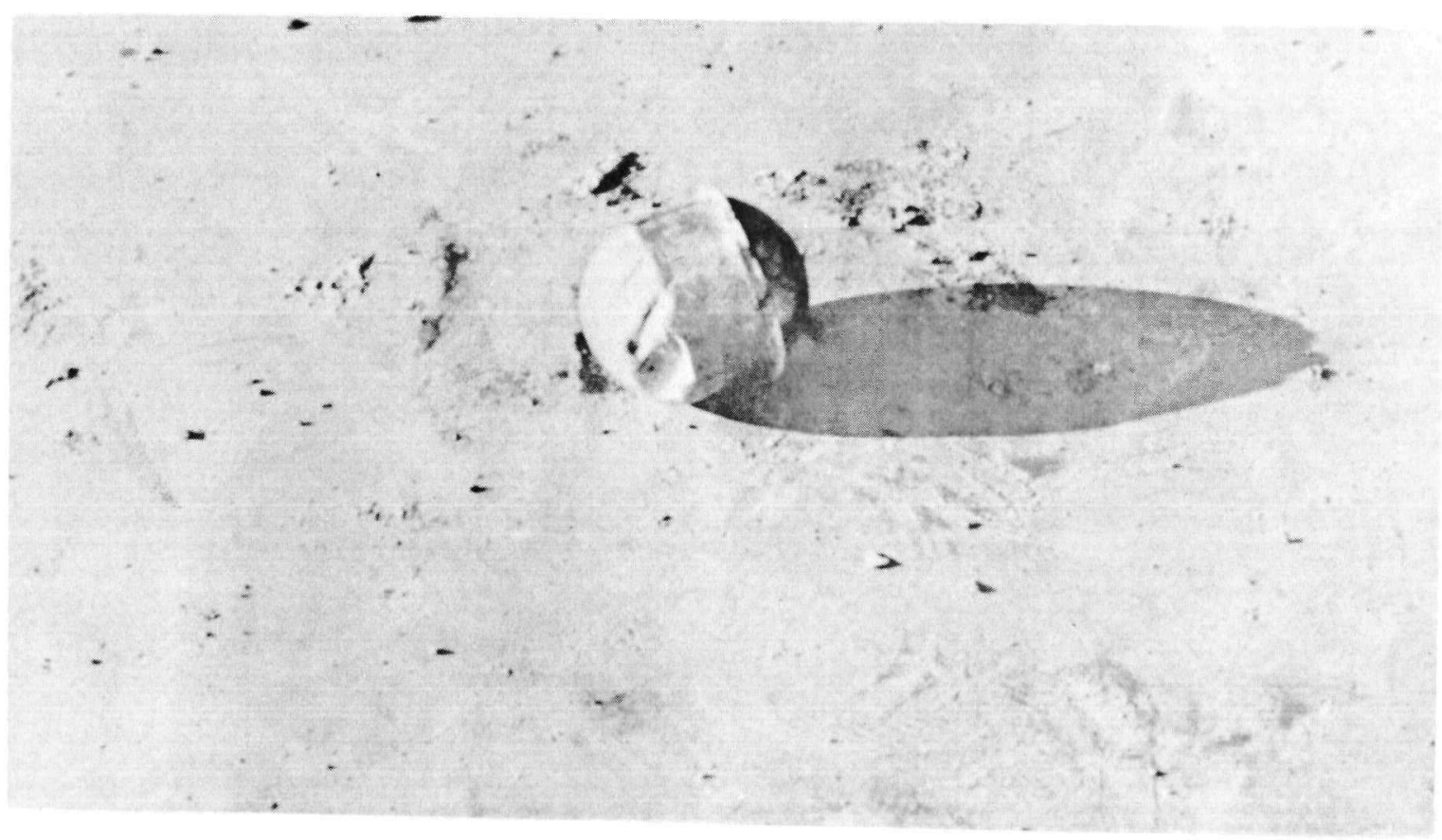

Fig. 60. High Velocity Impact Test--View of Specimen 5 After Impact on Water Target

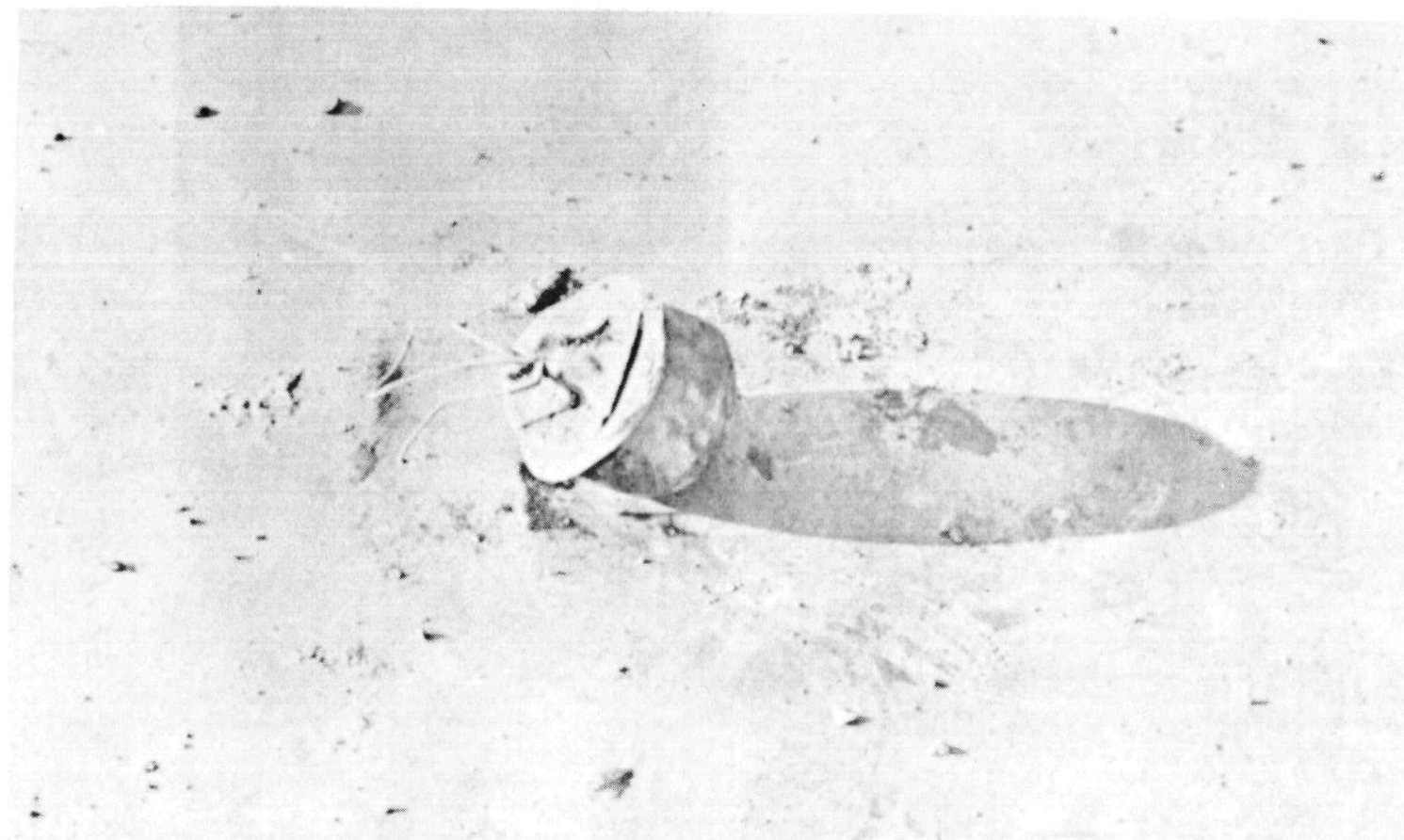

Fig. 61. View of Specimen 5 After Impact on Unconsolidated Rock Target 


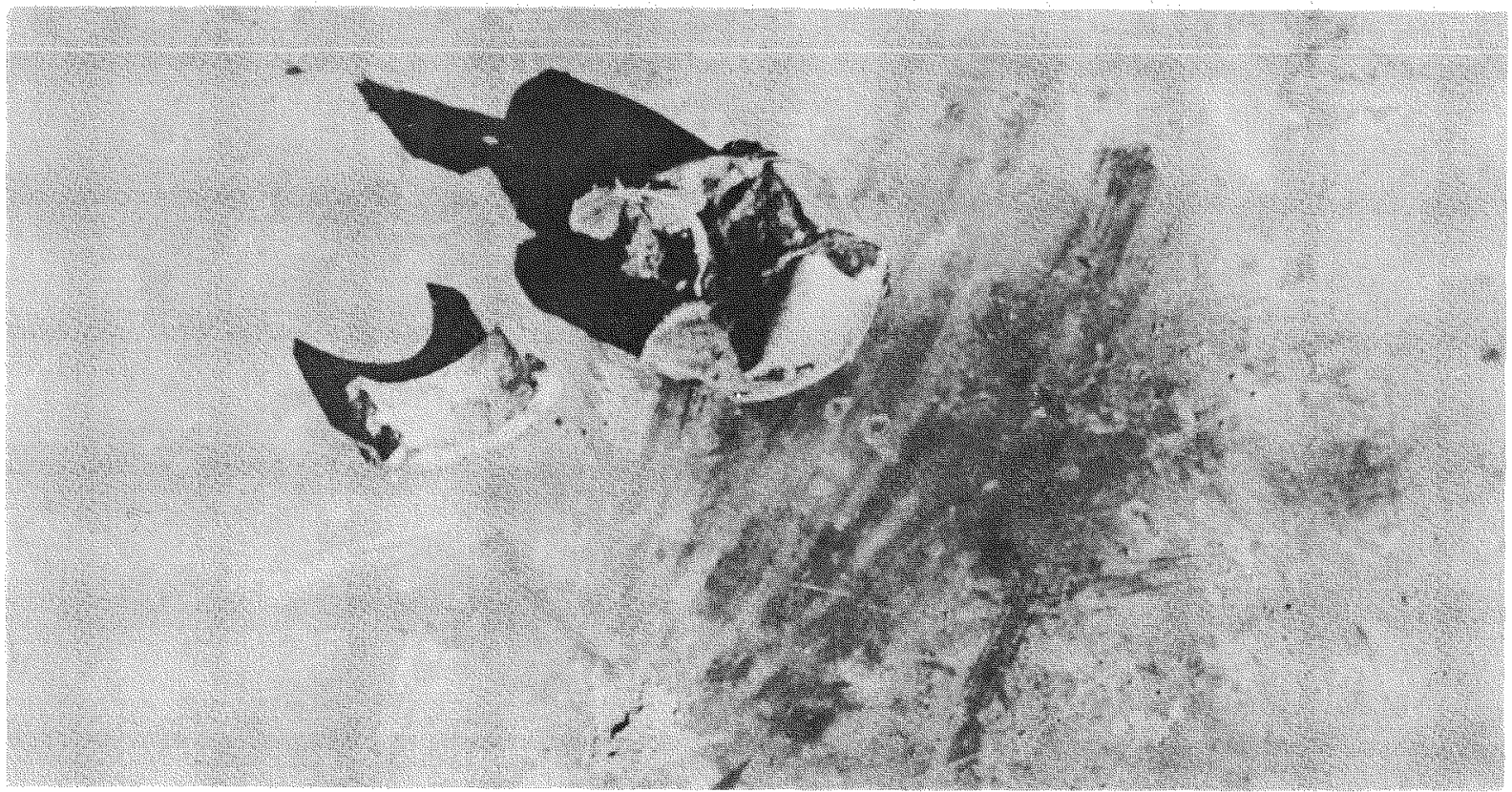

Fig. 62. View of Specimen 5 After Impact on Consolidated Rock Target

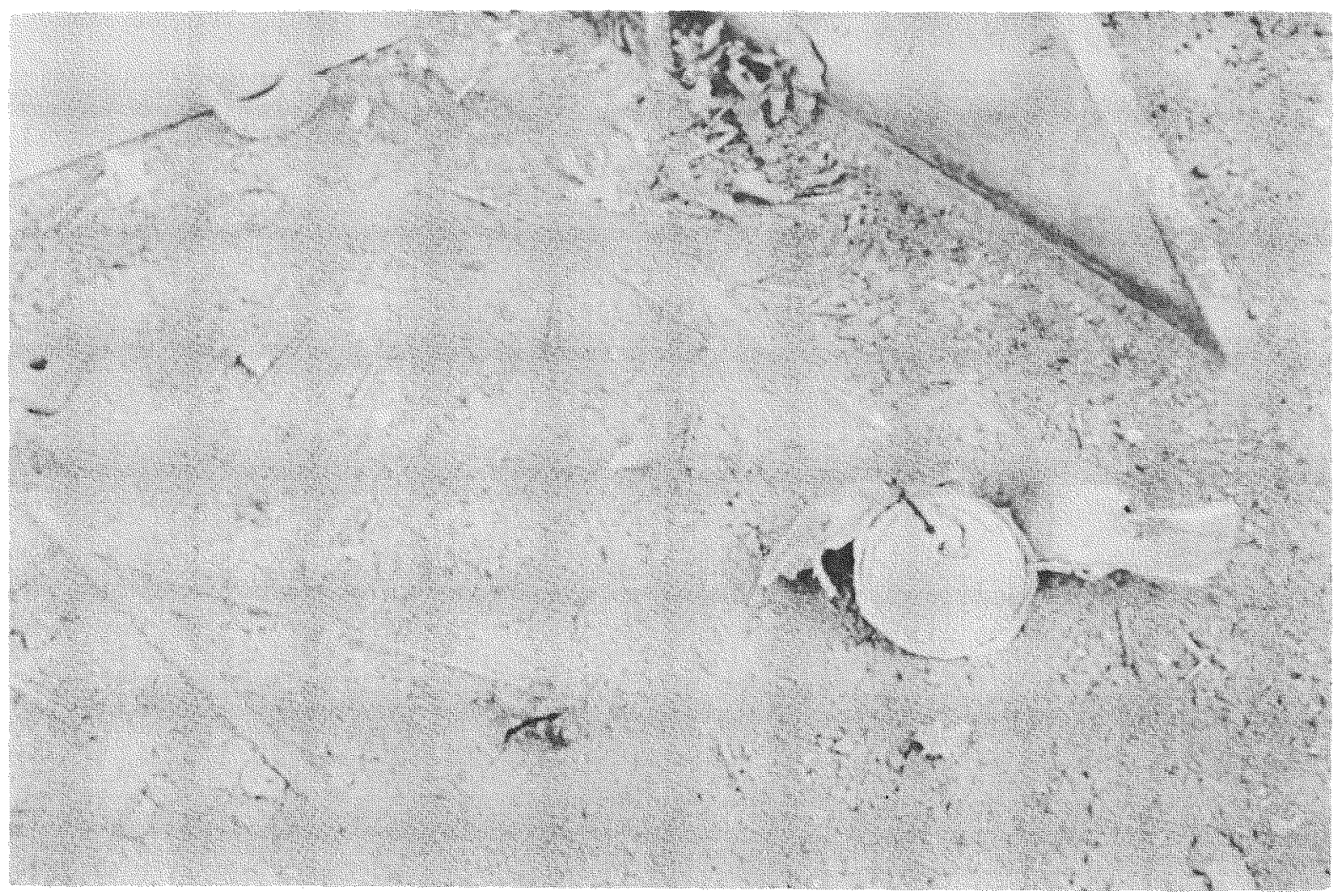

Fig. 63. View of Specimen 5 After Impact on Granite Block 


\section{TABLE ?}

Oxidation Test Results

\begin{tabular}{|c|c|c|c|c|}
\hline $\begin{array}{c}\text { Type } \\
\text { Material } \\
\end{array}$ & Atmosphere & $\begin{array}{l}\text { Test } \\
\text { Time } \\
(\mathrm{hr} r) \\
\end{array}$ & $\begin{array}{c}\text { Weight } \\
\text { Change } \\
(\mathrm{gm}) \\
\end{array}$ & Cleaning Method \\
\hline Haynes 25 & Air & 136 & +0.0065 & Chemically cleaned \\
\hline Haynes 25 & Air & 136 & +0.0061 & Chemically cleaned \\
\hline Haynes 25 & Air & 136 & +0.0061 & Degreased \\
\hline Haynes 25 & Air & 136 & +0.0055 & Degreased \\
\hline Type 304 & Air & 136 & +0.0018 & Chemically cleaned \\
\hline Type 304 & Air & 136 & +0.0010 & Chemically cleaned \\
\hline Type 304 & Air & 136 & +0.0011 & Degreased \\
\hline Type 304 & Air & 136 & +0.0011 & Degreased \\
\hline
\end{tabular}

Attempts to determine a corrosion rate on the samples resulted in insignificant change since it was impossible to remove the oxide from the samples without chemically dissolving some of the sample. The final test evaluation is based on ASTM method (181-50) which recommends visual and metallographic examination. The following conclusions can be drawn as a result of these examinations:

(1) The Haynes 25 specimens developed an oxide surface coat and showed an appreciable weight gain. However, metallographic examination proved the effect to be limited to the surface, and the oxide coat, once formed, appeared to inhibit further oxidation.

(2) The stainless steel specimens showed less weight gain but

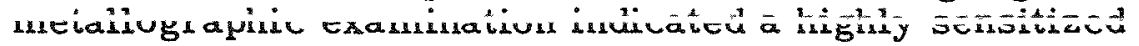
condition.

The corrosion test in salt water was concluded after 720 hours of exposure. Two samples each of Haynes 25 alloy and Type 304 stainless steel were tested. The samples were immersed in salt water in a plexiglass 
bath as shown in Fig. 64. A heat transfer analysis determined that the isotope capsule surface temperature would be $89^{\circ} \mathrm{F}$ when submerged in sea water at $68^{\circ} \mathrm{F}$. The temperature gradient was maintained by circulating warm tap water through the inside of the tubing while the bath temperature was maintained by a cooling coil. The specimens are currently being prepared for metallographic examination.

The corrosion tests of Haynes 25 alloy and AISI 304 stainless steel in salt spray were initiated after fabrication of the specimens and test fixtures. A salt spray cabinet was constructed of plexiglass with the atomizer mounted on the salt solution side of the divider wall. A cooling water jacket surrounds the salt spray box with tap water used for cooling. Two samples each of internally heated Haynes Alloy 25 and Type 304 stainless steel along with unheated controls of each metal are being tested. The Haynes Alloy 25 test specimens were formed by rolling 0.050 -inch sheet into $3 / 4$-inch $O D$ tubing and welding with parent metal weld rods. The tube bottoms were capped with Haynes 25 discs and welded. The Type 304 samples are 3/4inch $O D$ seamless tubing with the bottoms welded closed with Type 304 discs.

A heat transfer analysis to determine the surface temperature of the isotope capsule when exposed to air resulted in a predicted temperature of $657^{\circ} \mathrm{F}$. This analysis was based on radiative heat transfer to air at $68^{\circ} \mathrm{F}$. Conductive and convective heat transfer was neglected due to the difficulty of making reasonable assumptions of heat transfer coefficients for the possible surface variations. An assumed surface temperature of $600^{\circ} \mathrm{F}$ was maintained with variac-controlled Calrod heaters inserted into the tubes. Temperatures were calibrated with thermocouples before inserting the test devices into the salt spray chamber, since it was not desirable to run thermocouple wiring into the test chamber. The test arrangement is shown in Fig. 65. The test has been in operation for 673 hours to date and will be completed early in the next reporting period.

\section{d. Ejection}

A preliminary reusable ejection test fixture was designed and fabricated. Seven experimental snots were fired using a simulated generator mass to evaluate single and double squib operation, shear pin materials and vertical specimen travel. The tests were continued with a simulated generator specimen ballasted to 4.1 pounds. Based on previous test results, a series of five vertical shots resulted in an average vertical specimen rise of 49 feet using one T-6 aluminum shear pin and two M75 pressure squibs for all shots.

A ballistic analysis was performed to correlate the vertical rise in terms of range. Assuming a vacuum condition, a vertical rise of 50 feet is equivalent to the initial velocity required for a horizontal range of 200 feet with the thrust axis raised 45 degrees above the horizontal. The estimated air drag of the generator would reduce the range in the actual case by approximately $4 \%$. 


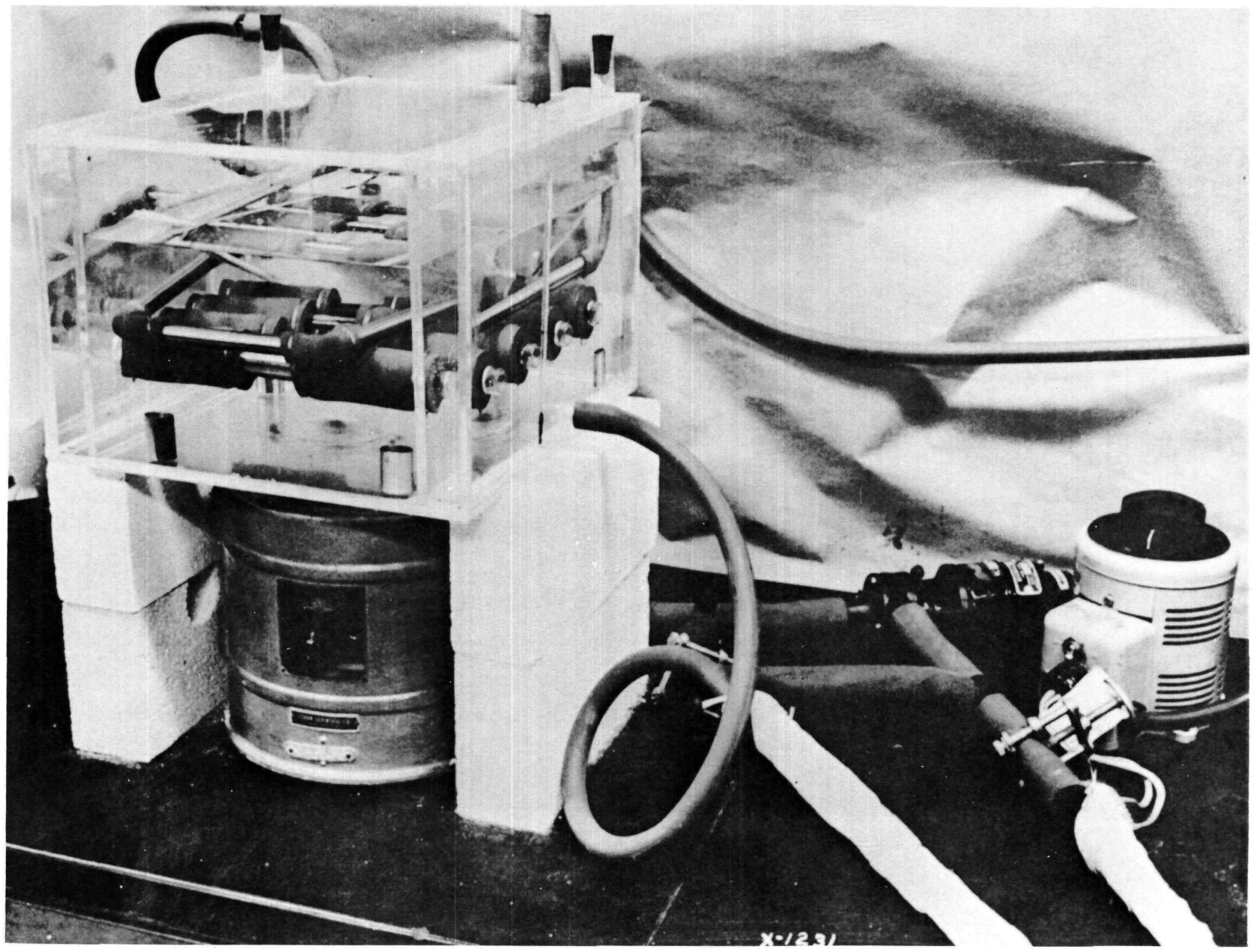

Fig. 64. Salt Water Corrosion Test Apparatus 


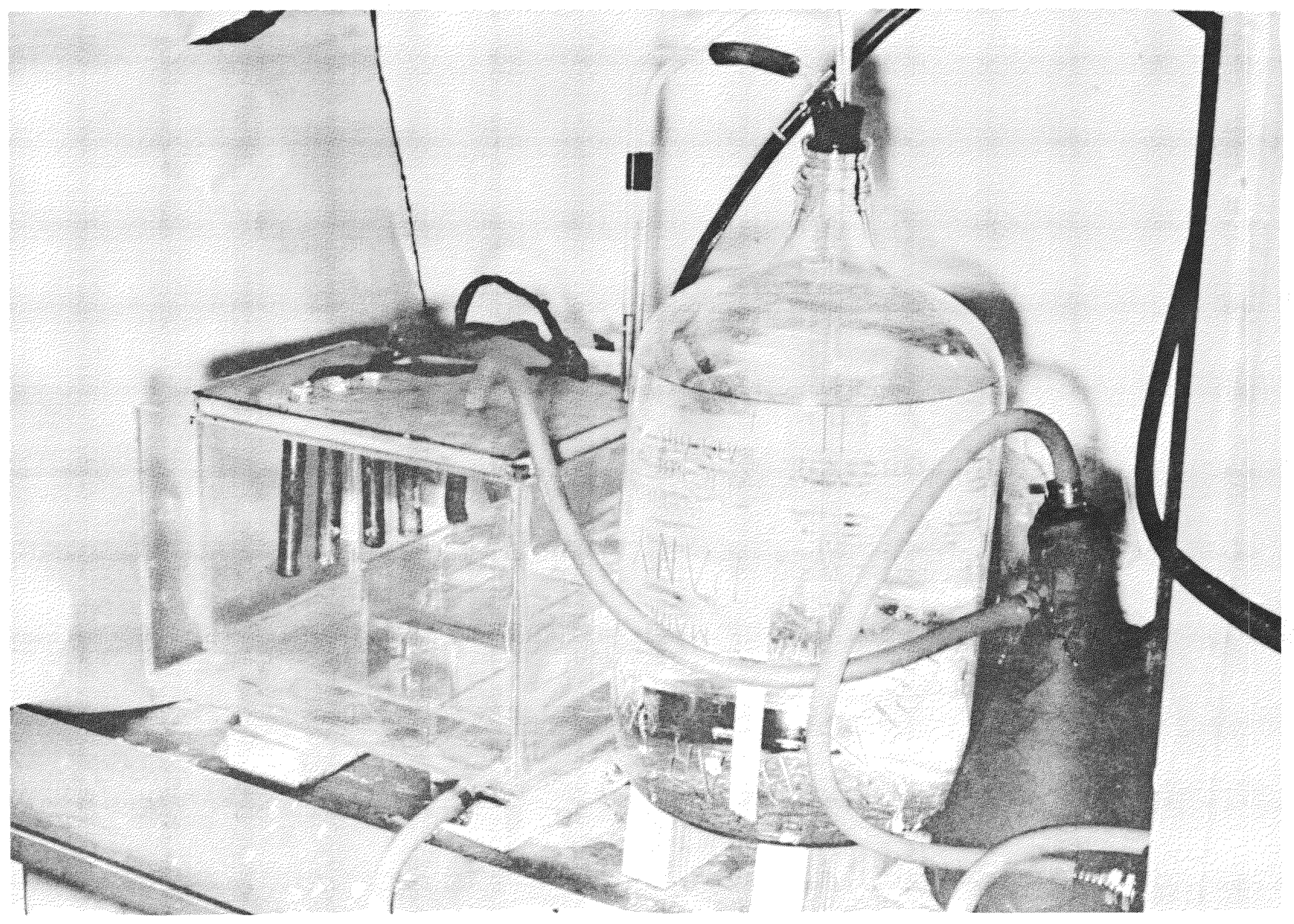

Fig. 65. Salt Spray Corrosion Test Apparatus 
To allow for this drag, charge variation, and projectile dispersion, the thruster design is currently being modified to optimize the pressurethrust relationship and effect a $15 \%$ increase in initial velocity. The thruster components, bearing cap, and specimen used are shown in Fig. 66. The bearing cap design proved to be adequate, since only a slight distortion occurred in the specimen copper shell.

\section{Re-entry Environments}

Normal operation of the vehicle system will place the payload and generator in orbit but re-entry from orbital altitudes must be assumed at any time during the planned operational life of the generator. The active radioisotope remaining must be dispersed during the re-entry phase through generator and capsule burnup. The re-entry environments will be simulated through plasma jet tests. A more positive control of isotope dispersal may be accomplished by destroying the containment capsule with a shaped charge. Tests to develop this destruction method are being conducted. The effect of the helium pressure buildup resulting from alpha emitting radioisotopes will be determined. Container specimens will be subjected to high internal pressure to define material stress rupture limits at temperature.

Plasma jet. An analysis of the aerodynamic burnup characteristics of the generator has been completed using a code programming method for machine calculation. Preliminary vehicle information was used to develop a synthetic trajectory yielding a polar orbit of 275 statute miles altitude from a Vandenberg Air Force Base launch site. The calculation results indicate that aerodynamic burnup on re-entry will occur above a safe altitude with the location dependent on the actual orbit achieved. Actual Discoverer vehicle information was received from Lockheed Missile Systems Division on December 2, 1959. These data differed from the preliminary information mainly in the orbit height achieved. Additional calculation runs were performed to incorporate these data in the input information for the plasma jet tests. The input information consists of a time history of temperature, velocity and heat input anticipated during re-entry.

A subcontract was negotiated with the General Electric Aeroscience Laboratory to conduct these tests in their plasma jet facility. The heat input will be simulated with scaled generator specimens and full-scale isotope container specimens. The subcontract agreement, including a statement of work, has been submitted to the New York Operations Office of the AEC and tests will begin as soon as approval is received. 


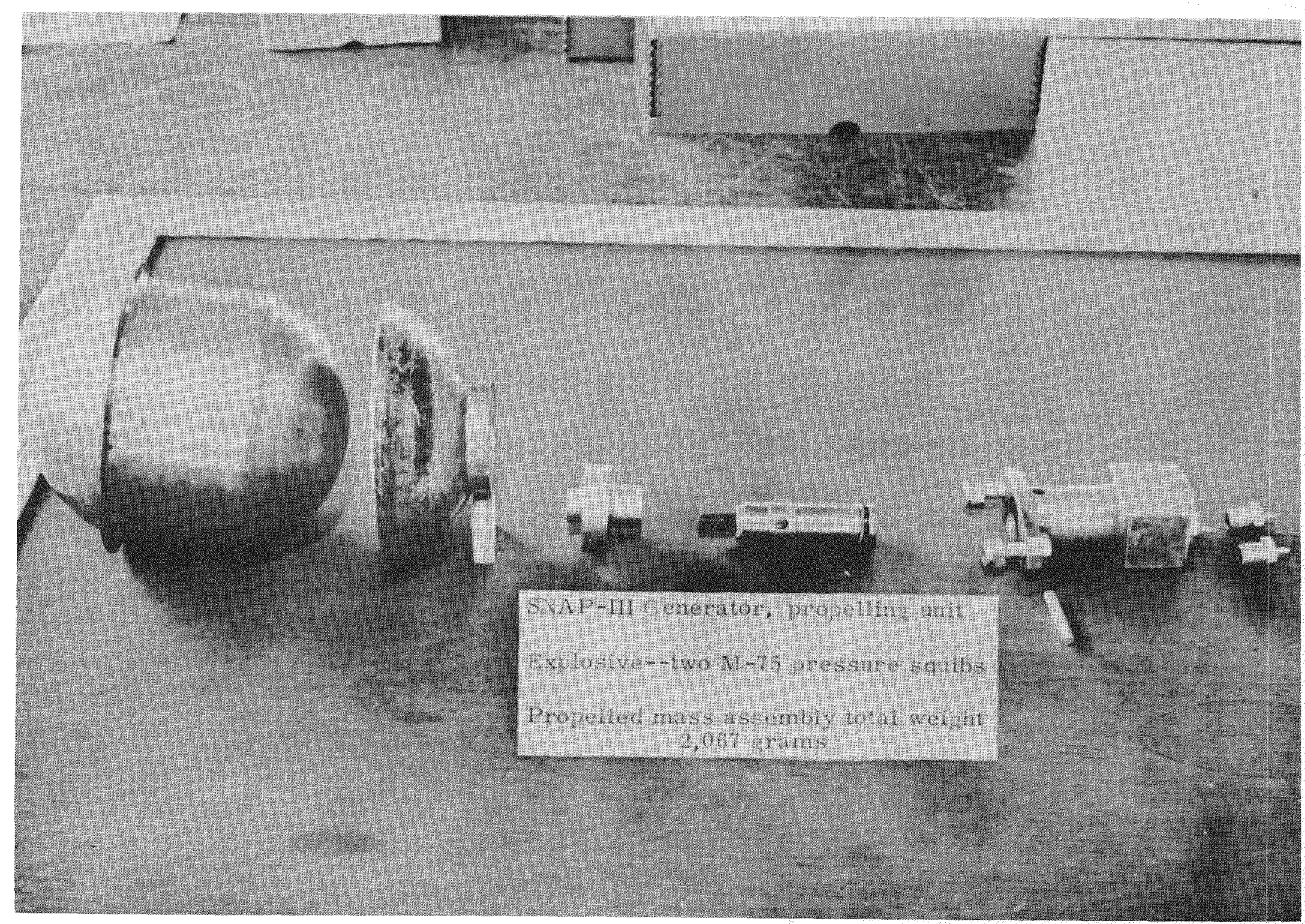

Fig. 66. Ejection Test Components 
Internal pressure. The stress rupture limits of the container material will be determined by subjecting container specimens to high internal pressures. The specimens will first be checked at normal operating temperature and maximum calculated internal pressure to determine seal integrity and creep rates if significant. The pressure and temperature will then be increased by stages to a maximum of 30,000 psi and $1700^{\circ} \mathrm{F}$ to determine failure conditions.

Fabrication of the test facility is complete except for the high pressure gauges. These items are on order and delivery is scheduled early in the next period.

Shaped charge destruction. A preliminary design of a shaped charge was completed based on the SNAP III-type generator configuration. The design objective is to vaporize the center portion of the container and radioisotope to achieve controlled dispersion.

Preliminary proof tests consisted of three shots to check design assumptions and determine optimum standoff distance between the charge and specimen. An analysis of the generator configuration indicated that 1.3 inches of steel would be an equivalent thickness. To better define penetration results, DuPont 16-A Jet Perforators were fired against a mild steel plate 2.5 inches thick with the following results:

\begin{tabular}{|c|c|c|c|}
\hline Test Number & $\begin{array}{l}\text { Standoff } \\
\text { (in.) }\end{array}$ & $\begin{array}{c}\text { Entrance Diameter } \\
\text { (in.) } \\
\end{array}$ & $\begin{array}{c}\text { Exit Diameter } \\
\text { (in.) }\end{array}$ \\
\hline 1 & 0 & 0.375 & $\begin{array}{l}\text { Incomplete } \\
\text { penetration }\end{array}$ \\
\hline 2 & 1 & 0.375 & 0.188 \\
\hline 3 & 2 & 0.375 & 0.188 \\
\hline
\end{tabular}

Figures 67 and 68 illustrate the entrance and exit side of the steel plate. The design proved adequate with a standoff distance of 1 inch.

Additional charges were ordered hut have not heen rereiver to date These charges will be directed against full-scale isotope capsules and simulated generator specimens as final proof-of-principle tests. 


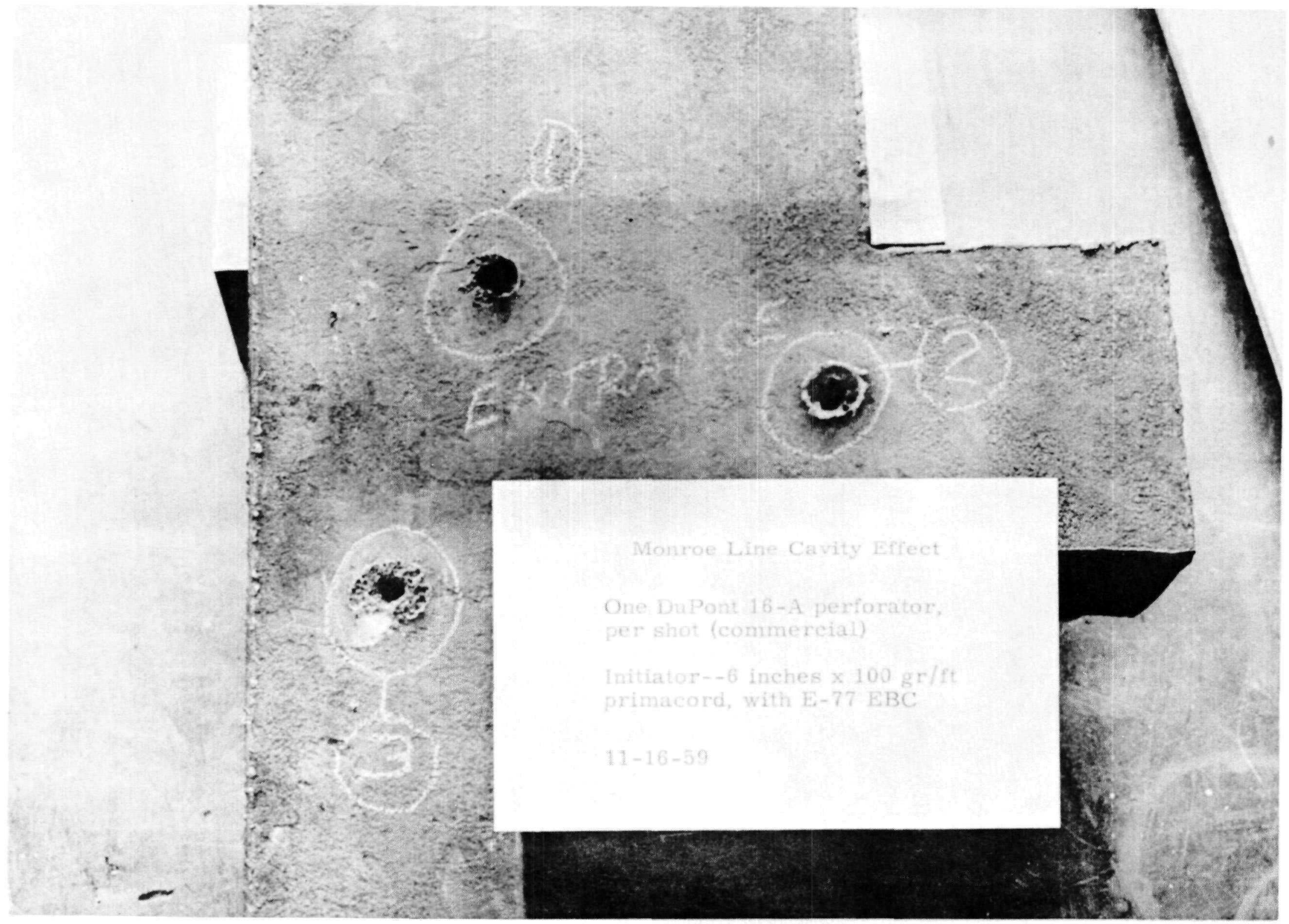

Fig. 67. Destruct Test Results--Entrance Side 


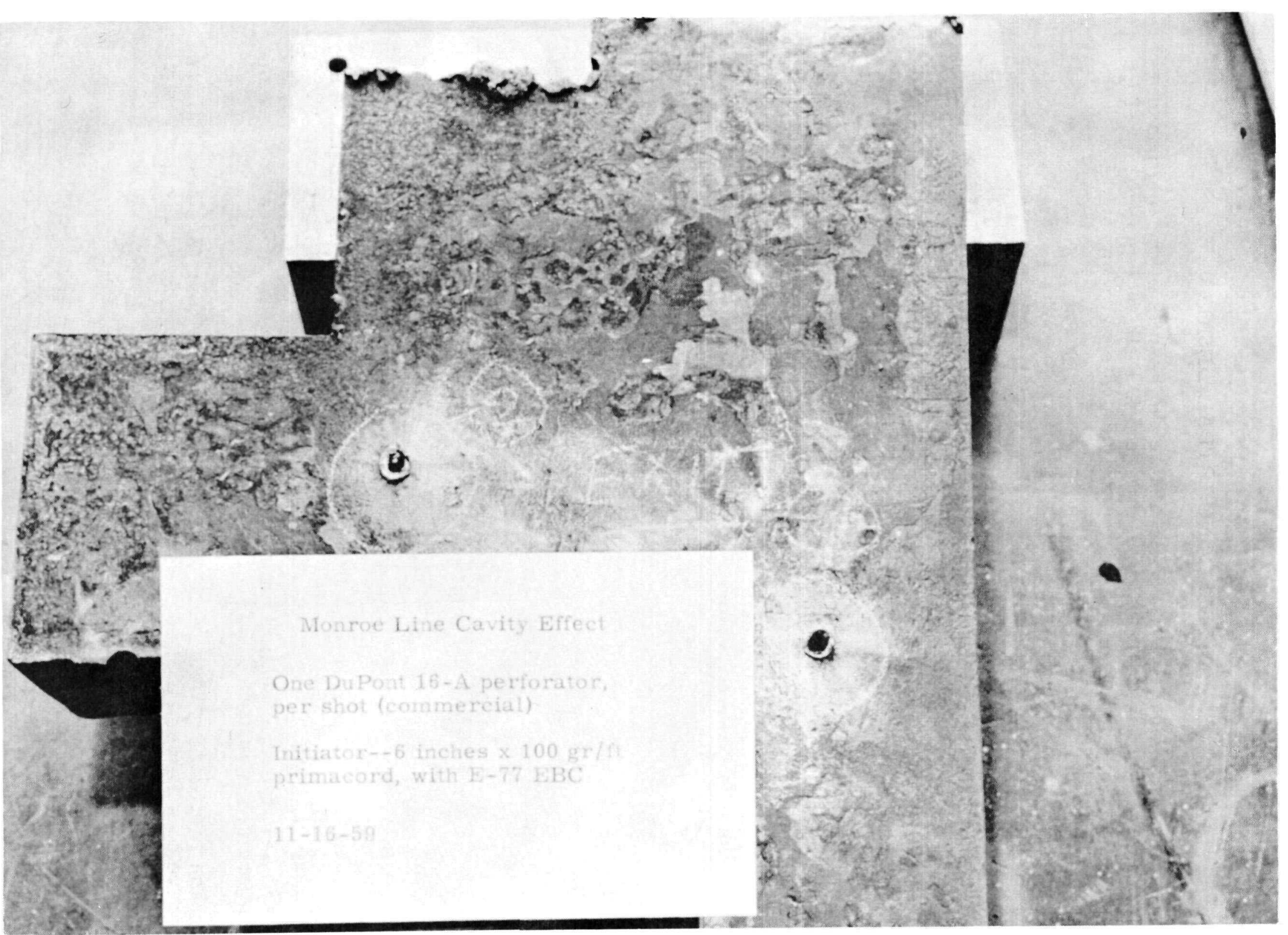




\section{B. SYSTEM CONCEPTUAL DESIGN}

The purpose of this phase is to utilize the results of the test phase to complete a conceptual design using the SNAP III-type generator. This will enable us to produce a short lead time system for any low power space application.

\section{Cerium-Fueled System}

The information for a topical report and a hazards analysis report for this system has been assembled but system evaluation must await completion of the test portion of this subtask.

2. Polonium-Fueled System

An investigation of the amount of molten lead existing in the polonium capsules was conducted to determine the possibility of internal corrosion. Using the decay rate of Polonium-210, the internal temperature of the fuel capsule and the quantity of lead present was computed as a function of time. The results indicate that approximately 0.17 grams of lead would exist within the capsule before the internal temperature decreased to the melting point of lead. These results will be used by the Materials Section to determine if the quantity of lead is significant from a corrosion standpoint.

A study of the temperature environment of a generator mounted in a missile nose cone was initiated based on the aerodynamic heat inputs during ascent. A set of differential equations defining the heat flow were derived but a solution using transform techniques has proven to be impractical due to algebraic complexity. A simplified analysis of the temperature environment is in the final phases and appears to offer a solution. The simplifying assumptions were (1) that no heat escapes from the generator surface during ascent and (2) that no heat is transferred from the nose cone material to the generator. The first assumption requires that the generator temperature be lowered artificially by external cooling methods to a point where all the isotope heat input will be absorbed in raising the temperature of the generator materials during ascent. The second assumption requires that sufficient insulation be incorporated in the nose cone structure so that the temperature rise of its internal surface due to aerodynamic heating matches approximately the generator surface temperature rise.

The preliminary data from the altitude chamber tests are being utilized to develop a revised plot of power output versus time using the input watage derived from the Polonium-210 decay curve. 
The internal pressure generated within the isotope container due to the formation of helium was calculated to be $6507 \mathrm{psi}$. This value is very conservative in that a temperature of $1100^{\circ} \mathrm{F}$ was assumed at infinite decay. The stress produced in the isotope capsule material was calculated and compared to the boiler code allowable stresses for pressure vessels. The maximum stress occurs at the inner wall of the tapered end as follows:

\section{(psi)}

Internal pressure- -6507 psi

Tangential tension stress 10,400

Radial compression stress

Shear stress 8,453

Assuming 10,000 psi external pressure from shock, these values become:

$$
\underline{(p s i)}
$$

Internal pressure- -6507 psi

Tangential compression stress

Radial compression stress

Shear stress

These stresses are well below the allowable stresses of Haynes 25 alloy. Based on an ultimate tensile strength of 90,000 psi, the maximum allowable external pressure is approximately 38,500 psi.

Various mounting arrangements incorporating ejection and destruction provisions are being studied to determine heat transfer characteristics since the explosive units are temperature sensitive. A preliminary weight estimate for a single generator installation is as follows:

\section{Item}

Structura] mounting

Destruct/ejection system

Bearing cap

Generator

\begin{tabular}{c}
$\begin{array}{c}\text { Weight } \\
(1 \mathrm{~b})\end{array}$ \\
\hline 0.70 \\
2.50 \\
0.45 \\
3.84 \\
\hline 7.49
\end{tabular}


3. Curium-Fueled System

No work scheduled this reporting period.

4. Converter and Battery System Studies

This study began with a literature search of battery performance and converter design information. 
Blank page 
VI. SUBTASK 5.6--1.0-WATT NUCLEAR POWER SUPPLY, SPACE APPLICATION*

The proposed power supply consists of a nuclear-powered generator, having an output of 1.5 watts at 1.5 volts dc, and a DC to DC static converter, with an output of 1 watt at 15 volts dc. Accomplishments to date include the establishment of the overall generator configuration, the sizing and arrangement of the $\mathrm{Pu}-238$ fuel, the analysis for the helium pressure buildup within the fuel encapsulation materials, the selection and sizing of the thermoelectric elements, the design of the radiator, the insulation arrangement and the thermal analysis of the overall configuration.

\section{A. DESIGN OF GENERATOR**}

1. Preliminary Configuration tion.

Table 8 lists specifications of the preliminary generator configura-

TABLE 8

Nuclear-Powered Generator Data

Generator Dimensions

Overall size

$4-5 / 16$ in. high

$6-1 / 4$ in. wide

Radiator area

$13-1 / 2$ in. long

$80-1 / 2 \mathrm{sq}$ in.

Electrical Conversion System

Lead telluride semiconductors

N-Type P-Type

Diameter

$0.250 \mathrm{in}$.

$0.250 \mathrm{in.}$

Length

$1.5 \mathrm{in.}$

1.5 in.

Number of elements

15

15

Radioisotopes

Plutonium -238

*D. Knighton

**J. Thomas 


\section{TABLE \& (continued)}

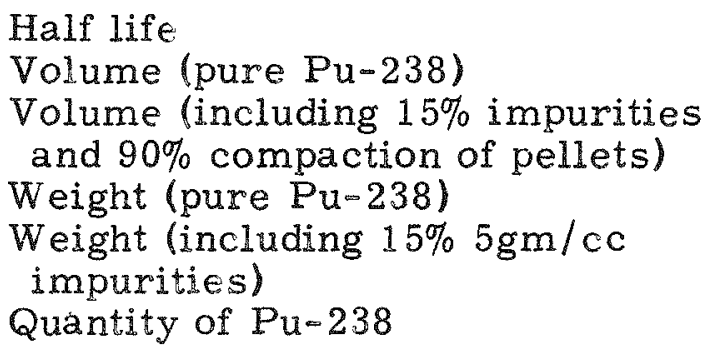

Thermal Properties

Input power

Hot junction temperature

Cold junction temperature

Electrical Properties

Output power

Voltage

Overall efficiency

Thermoelectric efficiency
86.4 years

$3.30 \mathrm{cc}$

$5.80 \mathrm{cc}$

$54.40 \mathrm{gm}$

$64.00 \mathrm{gm}$

940 curies

The generator consists of an encapsulated radionuclide heat source. a heat source support, a thermoelectric energy conversion system, a radiator, insulation and an outer housing. The support structure around the capsulated heat source is stainless steel sheet, and the outside container is formed of aluminum alloy sheet. The heat source consists of cylindrical Plutonium-238 pellets which have a length-todiameter ratio of 2 .

The unit has been designed for installation in the skin of a spherically shaped space satellite. The radiator of the generator forms part of a satellite skin surface and is insulated to eliminate high heat flow to the satellite electronic equipment. The generator output of 1.5 watts at 1.5 volts dc is fed into a static converter which powers the low power transmission equipment within the satellite.

Figure 69 is a drawing of this generator.

\section{Radioisotope and Containment}

The heat source material is Plutonium-238. This radioisotope has been selected for its 86,4 -year half life, its relatively high power density, its availability, and its low shielding requirements. Due to $\mathrm{Pu}-238^{\prime}$ s long 


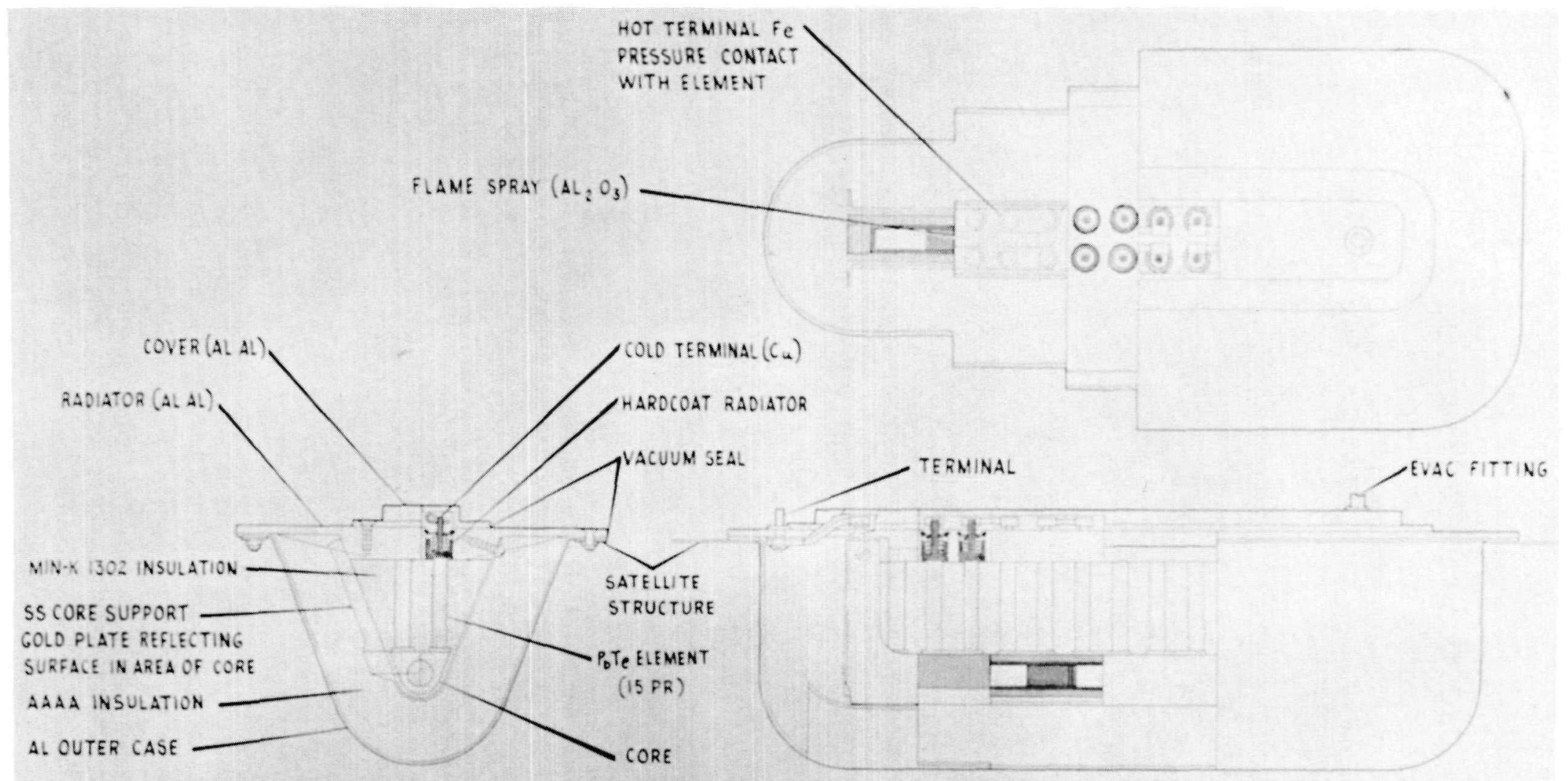

FUEL LOADING PLUG

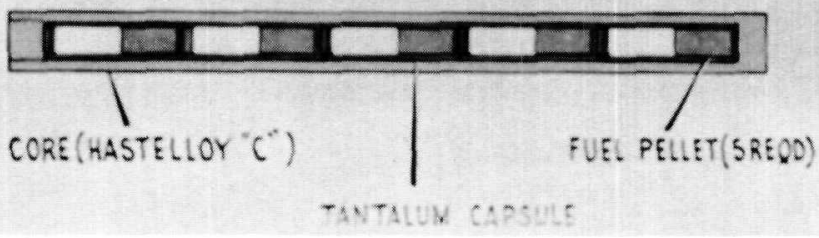

Fig. 69. Preliminary Generator Design 
half life, the power output will drop only $4 \%$ over a five-year period. This eliminates the need for a thermal control device so the generator will have no moving parts.

The calculations used to determine the volume and weight of $\mathrm{Pu}-238$ fuel are as follows:

The thermal watts required are:

Overall $\eta=4.9 \%$

Electrical power output $=1.5$ watts

$\frac{\text { Electrical power }}{\text { Overall } \eta}=\frac{1.5}{0.049}=30.60$ thermal watts.

The grams of $\mathrm{Pu}-238$ required are:

Volume $x$ Density

$\mathrm{Pu}-238$ power density $=9.3 \mathrm{w} / \mathrm{cc}$

$\mathrm{Pu}-238$ density $=16.5 \mathrm{gm} / \mathrm{sc}$

$$
\frac{30.61 \text { watts }(t)}{9.3 \text { watts }(t) / \mathrm{cc}} \times 16.5 \frac{\mathrm{gm}}{\mathrm{cc}}
$$

$3.3 \mathrm{cc} \times 16.5 \frac{\mathrm{gm}}{\mathrm{cc}}=54.4 \mathrm{gm}$ 。

To establish the final weight and volume of the fuel, an estimated $15 \%$ impurity at $5 \mathrm{gm} / \mathrm{cc}$ and a $90 \%$ compaction of fabricated fuel pellets gave the data below*

Weight of $\mathrm{Pu}-238+15 \%$ impurity $=\frac{54.4 \mathrm{gm}}{0.85}=64.0 \mathrm{gm}$

and the volume increase due to $5 \mathrm{gm} / \mathrm{cc}$ impurities is

$$
\frac{(64.0-54.4) \mathrm{gm}}{5 \mathrm{gm} / \mathrm{cc}}=1.92 \mathrm{cc} \text {. }
$$


Summarizing:

$\begin{array}{lcc}\mathrm{Pu}-238 & \frac{\begin{array}{c}\text { Volume } \\ (\mathrm{cc})\end{array}}{3.30} & \begin{array}{c}\text { Weight } \\ \text { (gm) }\end{array} \\ 15 \%-5 \mathrm{gm} / \mathrm{cc} \text { impurities } & \frac{1.92}{54.4} & \frac{9.6}{64.0}\end{array}$

The quantity of $\mathrm{Pu}-238$ in curies is

$54.4 \mathrm{gm} \mathrm{Pu}-238 \times 17.4 \frac{\text { curies }}{\mathrm{gm}}=940$ curies。

The $\mathrm{Pu}-238$ source material is encapsulated in two successive containers to prevent the release of contamination to the atmosphere during the prelaunch or launch phases of the satellite carrier. The first inner containers, as shown in Fig. 69 , consist of welded tantalum cylinders. The primary function of these containers is to prevent the plutonium metal fuel from alloying with the structural materials of the unit. The second capsule is a hollow metallic shaped structure which will take structural loadings. Materials for this second capsule are being investigated for the requirements of (1) remaining intact for all missile abort conditions and (2) burning up on a re-entry from orbit. The burnup requirement also applies to the tantalum container and the $\mathrm{Pu}-238$ fuel pellets.

\section{Helium Buildup Pressure}

Calculations to determine the maximum pressure buildup due to helium formation within the capsules have been completed. An equation relating material pressure and volume within the fuel capsule has been prepared to permit a stress analysis for various metallic fuel containers. The equation for determining the helium pressure with respect to time may be found in Appendix A.

\section{Electrical Conversion System}

The thermoelectric material used for the preliminary design is cast lead telluride which is produced in two types - - the negative type (doped with $0.03 \% \mathrm{PbI}_{2}$ ) and the positive type (doped with $1.0 \% \mathrm{Na}$ ).

In order to achieve a more efficient and more reliable element installa.

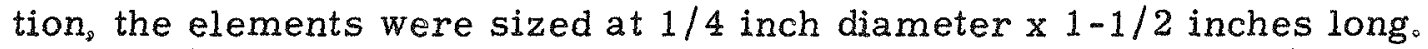
Figure 70 is a graph which was used to make a rapid selection of 
P-Type doped with $1.0 \% \mathrm{Na}$

$\mathrm{N}$-Type doped with $0.03 \% \mathrm{PbI}_{2}$

Hot junction temperature $--1000^{\circ} \mathrm{F}$

Cold junction temperature $-300^{\circ} \mathrm{F}$

$L / A=12.24 \mathrm{E} / W=L / 0.786 D^{2}$ $\mathrm{E}_{\mathrm{e}}=$ external voltage

$\mathrm{W}=$ power out in watts

$L=$ Length of $\mathrm{PbTe}$ element

$\mathrm{A}=$ Area of $\mathrm{PbTe}$ element

$\mathrm{D}=$ Diameter of $\mathrm{PbTe}$ element

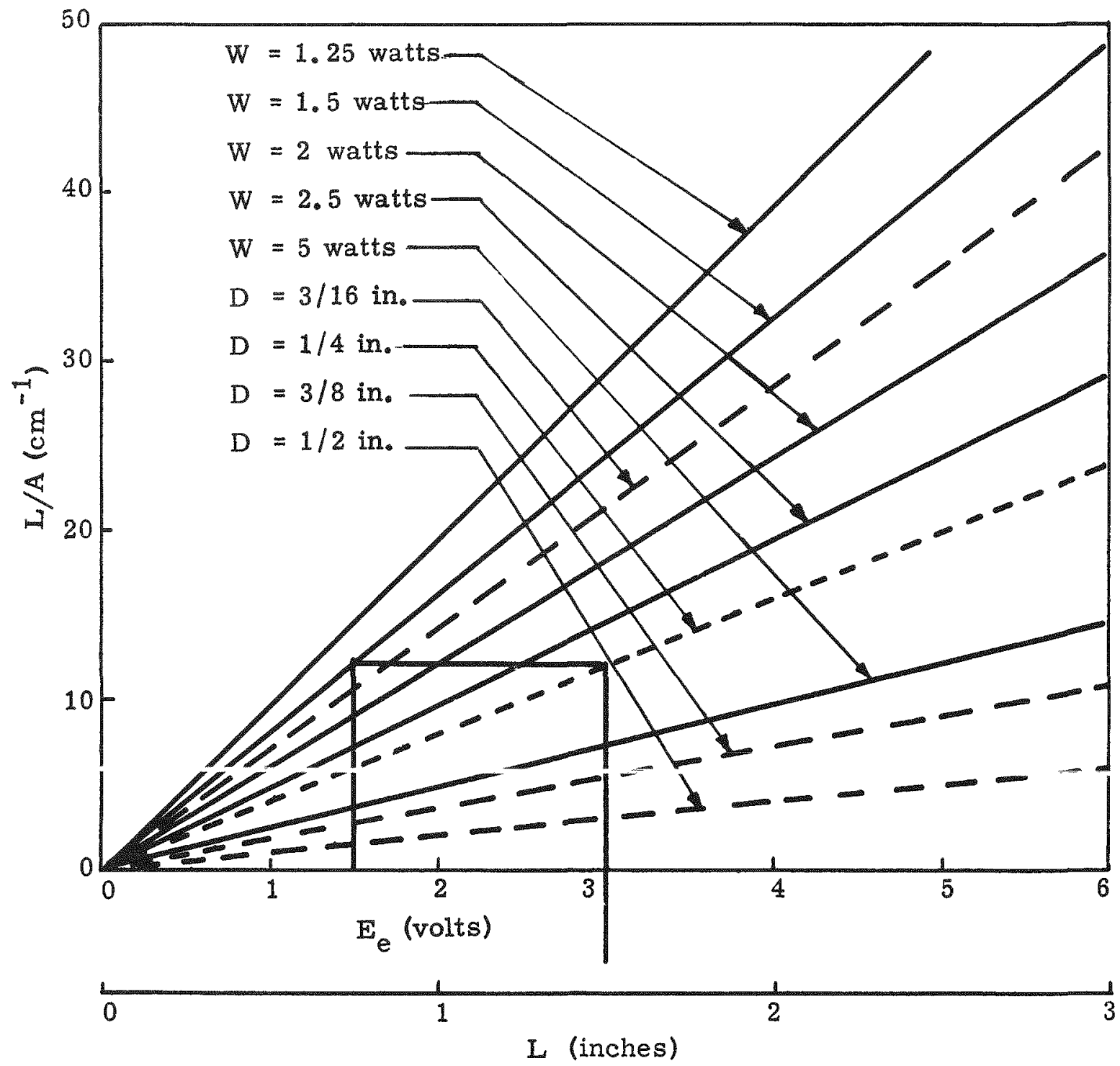

Fig. 70. Size of Lead Telluride Elements Versus Power and Voltage 
element size according to the power and voltage requirements. On the basis of theoretical data, 15 pairs of elements are required to produce 1.5 watts of electrical power at 1.5 volts de while operating at temperatures between 149 and $538^{\circ} \mathrm{C}$.

The thermoelectric elements have been placed between the flat surface of the encapsulated heat source and the radiator. To allow for thermal expansion and to maintain a good hot junction contact, the semiconductors are spring loaded on their cold junction ends. Electrical terminals from each element protrude through the radiator, simplifying electrical series connections. A cover over the exposed wiring hermetically seals the generator.

\section{Insulation Material}

Two types of insulating materials have been used in the design, a Johns Manville Min-K 1302 Rigid insulation and an Owens-Corning Fiberglas AAAA felted fiber insulation.

The Min-K 1302 insulation has been used in the areas around the elements to provide a stronger element installation. The load absorption of this insulation has been successfully proven on SNAP III shock, vibration and acceleration tests and is expected to give the same performance in this design. Besides having a reinforcing trait, the Min-K 1302 is readily fabricated into various shapes.

For the nonstructural areas of the generator design, the AAAA felted fiber insulation is used due to its lower conductivity value at a pressure of 100 microns.

Thermal insulation is also achieved by the use of a gold reflecting surface at the back side and end of the encapsulated fuel. This reflector is used to obtain a temperature drop between the source surface and the support structure, which reduces heat flow (or loss) to the radiator via the support structure. Figure 69 shows the position of the support structure and the area of gold plating.

\section{B. THERMAL ANALYSIS*}

Heat flow calculations for the generator package shown in Fig. 69 are contained in Appendix B. Figure B-1 in Appendix B shows temperatures at various points inside and outside the generator. 
The basic requirement in this generator concept is that the hot and cold junctions of the thermoelectric elements be maintained at $1000^{\circ} \mathrm{F}$ $\left(811^{\circ} \mathrm{K}\right)$ and $300^{\circ} \mathrm{F}\left(422^{\circ} \mathrm{K}\right)$, respectively. The heat source must, therefore, be at a temperature greater than $811^{\circ} \mathrm{K}$. It is assumed that the temperature of the source surface opposite the hot junction surface is $850^{\circ} \mathrm{K}$ 。 It is also assumed that the internal satellite structure is at a uniform temperature of $300^{\circ} \mathrm{K}\left(80^{\circ} \mathrm{F}\right)$. Other assumptions used in the calculations are described in Appendix B.

A summation of heat losses will give the required heat source power. The calculated heat losses are as follows:

Heat losses through elements to radiator Watts

Heat losses through Min-K to radiator 17.36

Heat losses through structure to radiator 2.73 Heat converted to electrical energy

Other heat losses (to interior of satellite)

1.50

0.83

\section{Total}

30.61

28.28 watts of this total flows to the radiator and must be dissipated into space.

In checking the adequacy of the radiator, the heat energy absorbed from the sun and earth must be considered. The condition at which the generator will operate at its lowest efficiency is when heat absorbed is at a maximum. This is when the radiator surface is normal to the radiation of both the sun and earth. The other extreme of zero energy absorbed occurs when the satellite is in the shadow of the earth.

For the analysis of this conceptual design, it is assumed that the radiator is completely insulated from the satellite skin. The heat energy is dissipated by radiation from the external surface of the radiator. For the first extreme condition, the total power absorbed in is calculated from

$$
W=A\left[1400 \alpha v\left(\mu+\frac{\alpha F}{\pi} \cos \ddot{b}\right)+e_{I R} 1 \dot{0} \bar{b} . \bar{b}\right]
$$

(Ref: Part III "Radiation Equilibrium and Temperature," by Goldman and Singer, ARS)

where

$\mathrm{W}$ = watts absorbed 


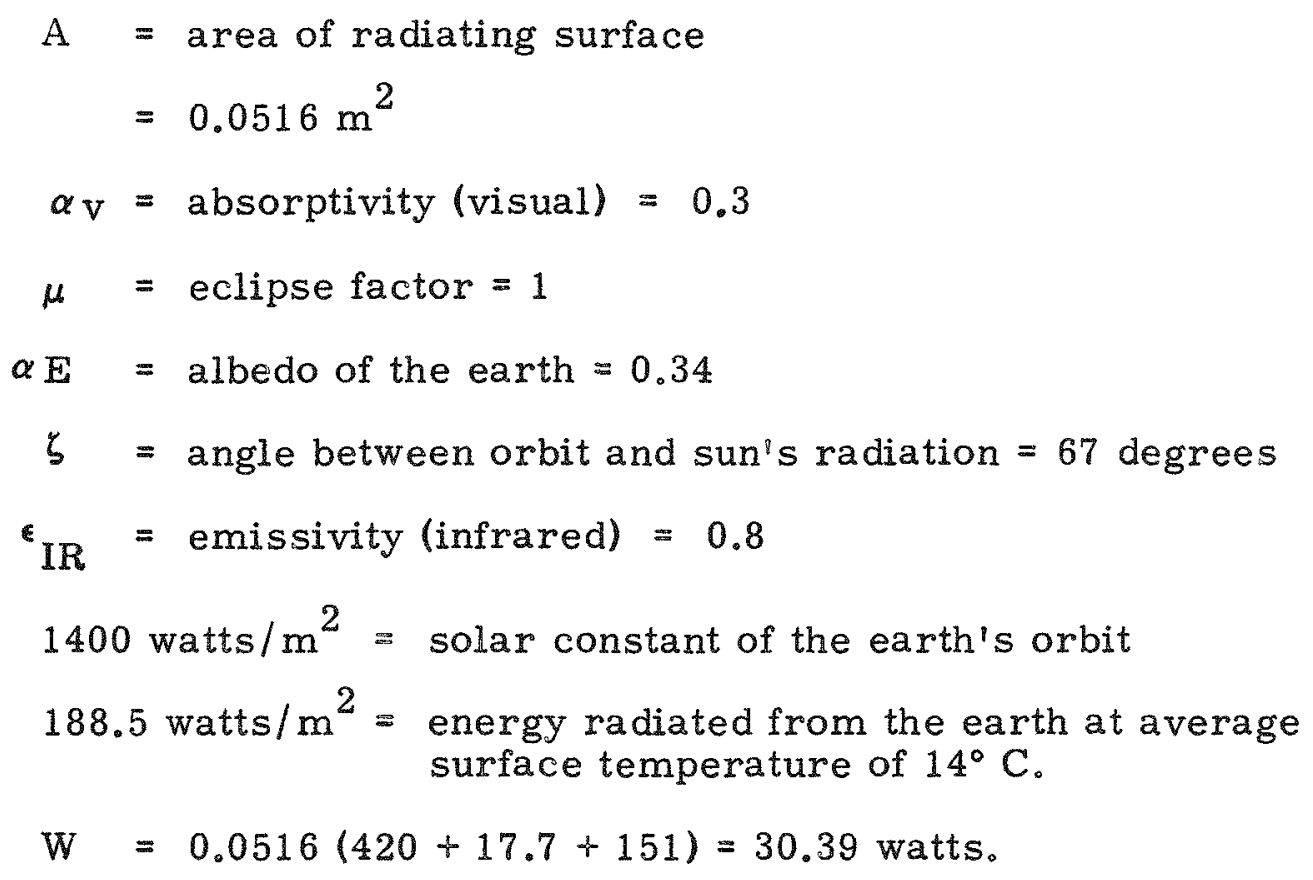

At equilibrium, the absorbed energy is equal to the thermal radiation from the radiation surface.

$$
\begin{aligned}
q_{T} & =28.28 \text { (internal source) }+30.39 \text { (absorbed) } \\
& =58.67=A \epsilon_{I R} \sigma T_{R}^{4} \\
A & =\text { area of radiating surface } \\
& =0.0516 \mathrm{~m}^{2} \\
\epsilon_{I R} & =\text { emissivity }=0.8 \\
\sigma & =5.67 \times 10^{-8} \text { watts } / \mathrm{m}^{2}-{ }^{\circ} \mathrm{K}^{4} \\
T_{R} & =\text { average temperature of radiator surface. }
\end{aligned}
$$

From this relationship, the average temperature of the radiator can be obtained by

$$
T_{R}=\left(\frac{q_{T}}{A \cdot I R}\right)^{1 / 4}=398^{\circ} \mathrm{K}
$$


For the case where heat energy is not absorbed, $\mathrm{T}_{\mathrm{R}}=341^{\circ} \mathrm{K}$. The average temperature of this particular radiator, therefore, will vary $57^{\circ} \mathrm{C}\left(135^{\circ} \mathrm{F}\right)$ from one extreme condition to the other. Since the cold junction temperature is $422^{\circ} \mathrm{K}$, the allowable temperature drop through the radiator is between $24^{\circ} \mathrm{C}\left(75^{\circ} \mathrm{F}\right)$ and $81^{\circ} \mathrm{C}\left(175^{\circ} \mathrm{F}\right)$. The final radiator design must be based on orbital conditions of a particular satellite in order to arrive at an optimum radiator design.

The temperature distribution on the radiator was calculated only for the condition of maximum energy absorption. The isotherms are shown in Fig. B-1 under Appendix B. The calculations were made by use of a two-dimensional relaxation technique for a steady-state condition. 


\section{SUBTASK 5.7--100-WATT THERMOELECTRIC GENERATOR CONCEPTUAL DESIGN*}

The objective of this task is to select and develop a conceptual design of a thermoelectric generator which will provide 100 watts of unregulated electric power throughout a six-month operational period in space. The heat source is Curium-242; this alpha emitter permits very modest biological shielding thicknesses and is much less expensive than Polonium210, Plutonium-238, etc. The generator is to meet as closely as possible the following general design conditions: reliability, including ability to withstand launching environmental shock, acceleration, and vibration; minimum biological hazard in normal operation, ground handling, and in case of abort; light weight; maximum efficiency and, hence, minimum isotope investment; and ease of fabrication and adjustment.

The areas of investigation for accomplishing these objectives include thermoelectric element and array analysis, heat transfer analysis, generator configuration and component design studies, thermoelectric materials tests, shielding analysis, and hazards analysis. Specific methods and results in each area will be discussed.

Prior to October 1959, the principal effort concerned the analysis of the generator with radiative heat transfer away from the cold junctions and with the biological shield contained, before launch, entirely within the hot junction surface. The results, already presented in the previous Quarterly Report, MND-P-3008, are summarized in the next section.

\section{A. THERMOELECTRIC AND HEAT TRANSFER ANALYSIS}

Three general configurations were originally proposed for the $100-$ watt thermoelectric generator. The first of these consisted of a radiative boundary-type structure which would entirely contain the liquid biological shield prior to launch. The shield would be located between the heat source and the hot junctions.

The second type of generator differs from the first in that its design is based on minimum weight with no consideration given to the biological shield location. The resulting shield consists of two parts: an internal shield between the heat source and the hot junctions, and an external shield outside the generator. Work is progressing on this investigation. So far, the general configuration has been established.

The third type uses forced convection of water to cool the cold junction and transfer the heat to a radiator located on the satellite skin. 
For the forced convection design, a 5-foot diameter cylindrical satellite body was considered using from 1.5 feet to 2.5 feet of the height as a radiating area. Laminar flow was assumed in order to reduce pumping requirements. It was shown that the laminar flow of an ideal incompressible fluid in a space filled by the fluid is independent of gravity. This investigation showed that a forced convection system would result in a lower cold junction temperature than would radiative cooling, thus increasing generator efficiency.

The surface temperature of the satellite skin was determined by Singer's equation. Using this temperature as the radiator temperature, a series of curves was obtained interrelating radiator area, cold junction temperature, flow rate, and number of flow tubes. These data are summarized in Fig. 71 .

In an effort to improve the performance of the convective boundary generator, heat transfer fluids other than water were examined. High specific heat was obviously desirable in order to reduce the pumping rate. Since the minimum weight design for water indicated a condition near the transition from laminar to turbulent flow, a medium with a low coefficient of viscosity was sought. Allyl alcohol, amyl alcohol, tertiary amyl alcohol, butyl alcohol, ethyl alcohol, isoamyl alcohol, isobutyl alcohol, methyl ethyl ketore, palmitic acid and ethyl ether were considered, but none permits a lower pumping rate or system weight than water.

The weight of the convective boundary generator is approximately 125 pounds. This excessive weight together with the reliability problem introduced by the pump, indicated that this design is not satisfactory.

The generator with direct radiation of heat from the cold junctions into space and with all biological shielding (required before launch) contained between the heat source and the hot junction surface, has already been analyzed. The analysis is described in detail in MND-P2229, "100-Watt Thermoelectric Generator, Preliminary Heat Transfor Analysis," and the mast impartent resulte aro given in Tahlo a

The configuration receiving chief attention during this period also depends upon the radiation of heat directly from the hot junctions to space, but the space between the heat source and the cold junction surface is now established by optimization of the power-to-weight ratio. Where this space is insufficient to include the necessary biological shielding, an external auxiliary shield is provided. The container of the auxiliary shield is integral with the final stage of the launch vehicle.

The configuration used in the preliminary analysis consists of three concentric spheres: heat source, hot junction, and cold junction. Although fabricability may not permit construction of a generator of this 

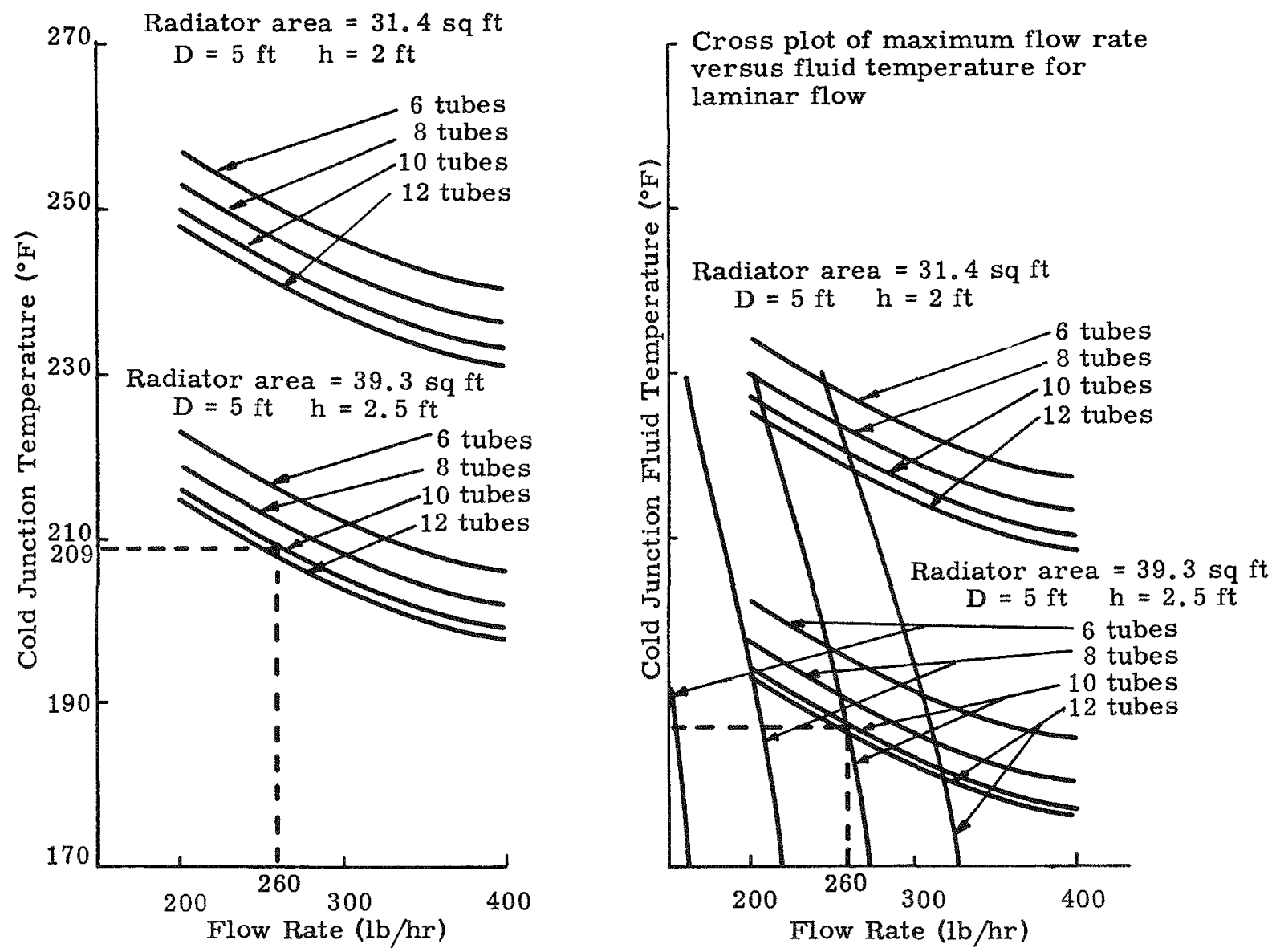

All tube diameters considered to be 0.25 ID Example:

1. Choose radiator area $(39.3 \mathrm{sq} \mathrm{ft})$ and number of tubes (10)

2. Locate fluid temperature $\left(187^{\circ} \mathrm{F}\right)$ and flow rate $(260 \mathrm{lb} / \mathrm{hr})$ on RHS with flow rate pickoff cold junction temperature on LHS

Fig. 71. Cold Junction Temperature and Cold Junction Fluid Temperature Versus Flow Rates 
TABLE 9

100-Watt Generator Specification and Data

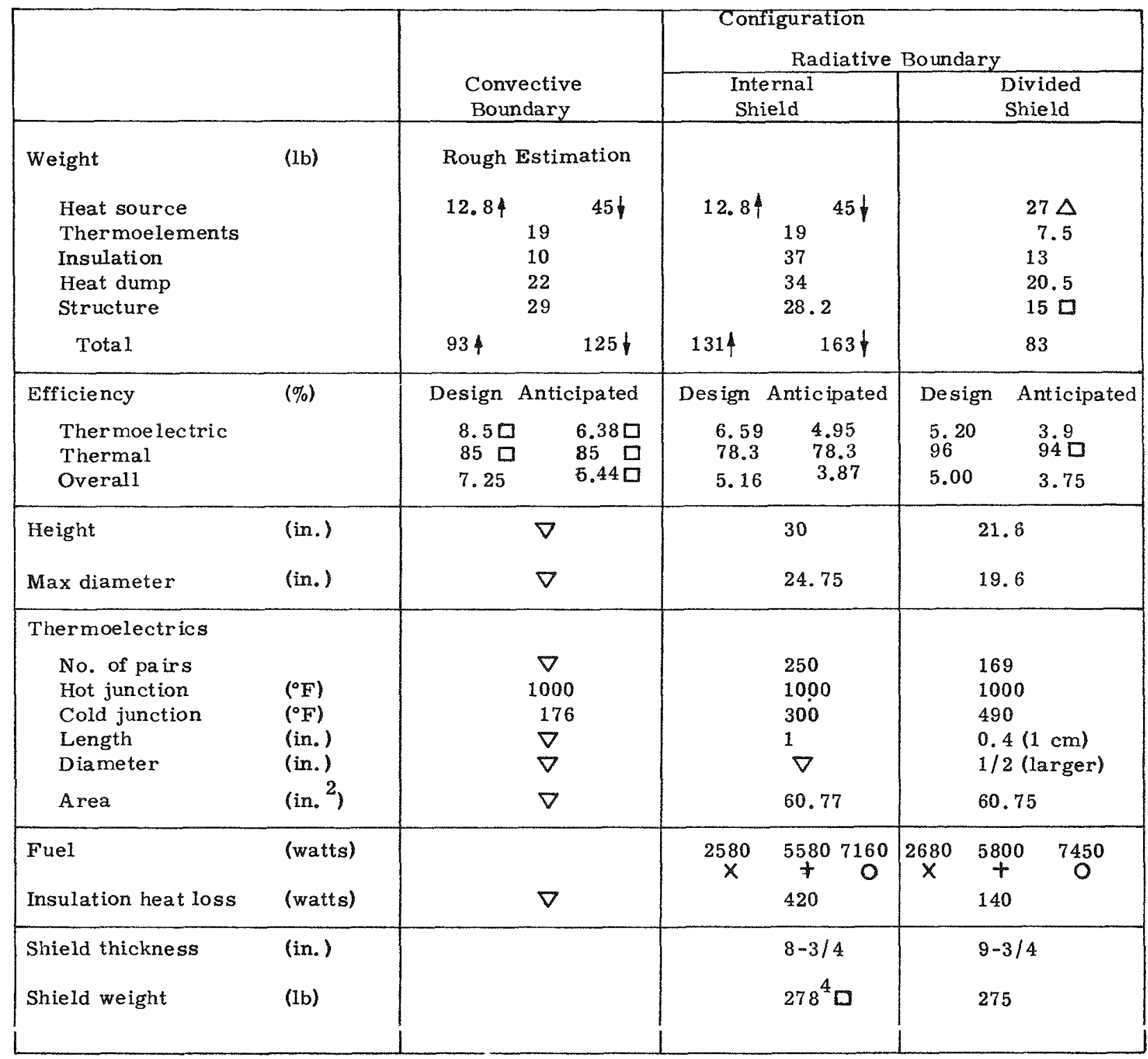

\section{With ablative}

1 Without ablative

$\Delta$ Should be decreased appreciably by design improvement

D Estimated

$\nabla \quad$ Not calculated

$X$ End of life

+ At launch

o Initial 
exact configuration, any necessary distortion should not seriously affect the calculations. Thus, an idealized optimum design was de termined which guided the choice of parameters in the actual design.

Preliminary investigations, consisting of manual calculations to check the machine procedure, showed a weight of approximately 70 pounds required to produce 100 electrical watts at 28 volts. The investigations showed that this optimum has a cold junction temperature of approximately $400^{\circ} \mathrm{F}$ to $550^{\circ} \mathrm{F}$.

Results of calculations of thermoelectric efficiency and generator weight for various radiator temperatures and thermoelement lengths are plotted in Figs. 72 and 73 . The results for the configuration chosen as optimum are listed in Table 9.

To optimize the radiative boundary configurations in a reasonable period of time, a program for the IBM-709 digital computer was developed. This program was used to obtain the results plotted in Figs. 72 and 73. A detailed description of the program, including a printout of the object deck, may be found in Appendix C.

The program just described is applicable only to generators with concentric spherical isothermal surfaces, and it assumes that thermoelement characteristics are functions of the hot and cold junction temperatures alone. A more accurate and more widely applicable method of analysis is also being developed. This effort is supported partly under this subtask, partly by other tasks in this contract, and partly by The Martin Company.

The code requires as input data the hot and cold junction temperatures of each thermoelement, the length of the elements, and the electrical resistivity, thermal conductivity, and Seebeck coefficient of the thermoelectric materials as functions of the temperature. The program is entirely rigorous and general, with the restriction that the current in each element satisfy the continuity equation. The output data a re the radii of the elements, number of pairs, and weight of elements for optimum thermoelectric efficiency.

The boundary conditions were established, a difference technique was established, and the coding process was begun.

To establish the isotope fuel form and the design of the heat source, centerline temperatures of fuel forms were calculated. This temperature must be below the melting point of the fuel compound. 


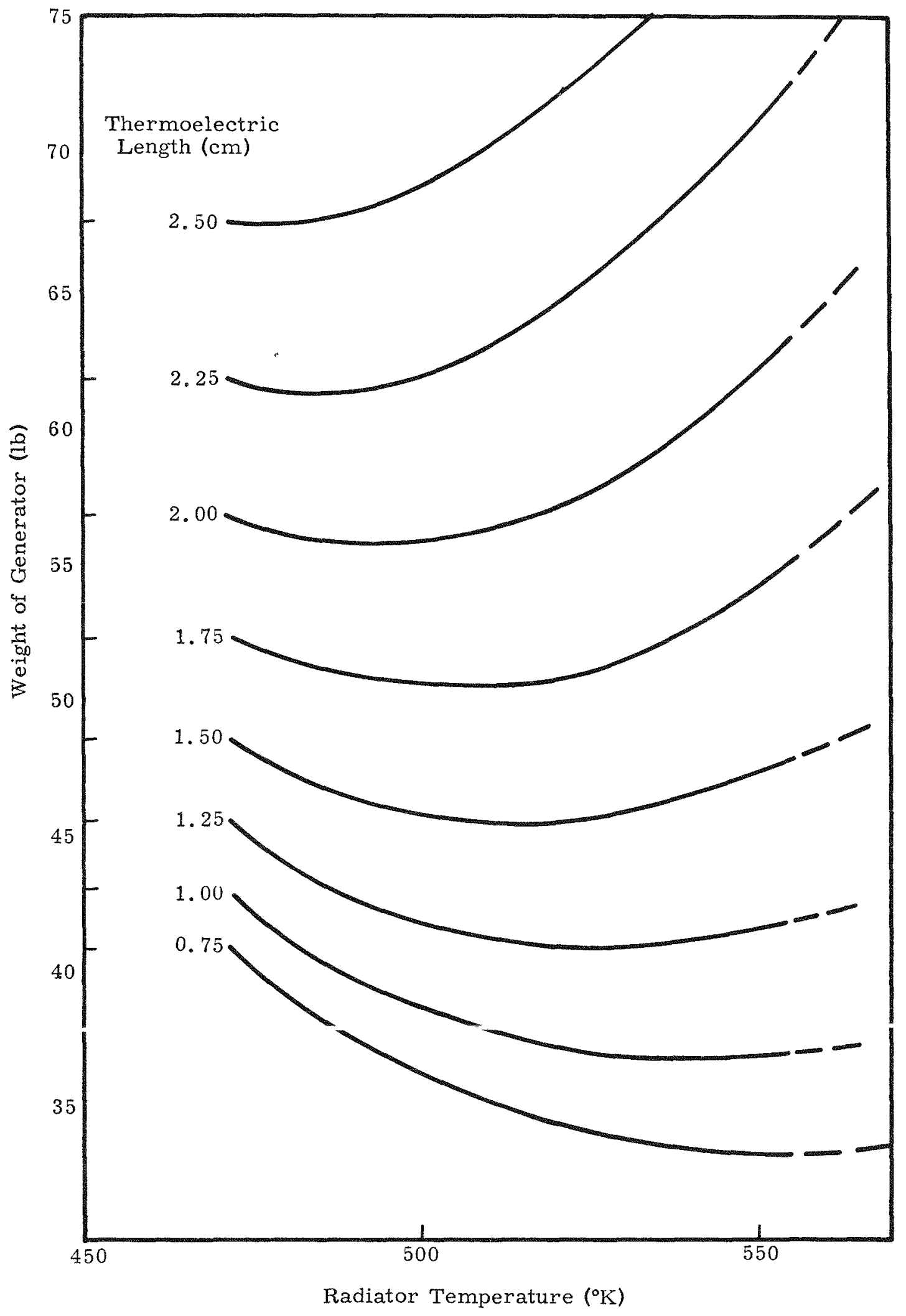

Fig. 72. Variation of Generator Weight with Radiator Temperature and Thermoelectric Length 


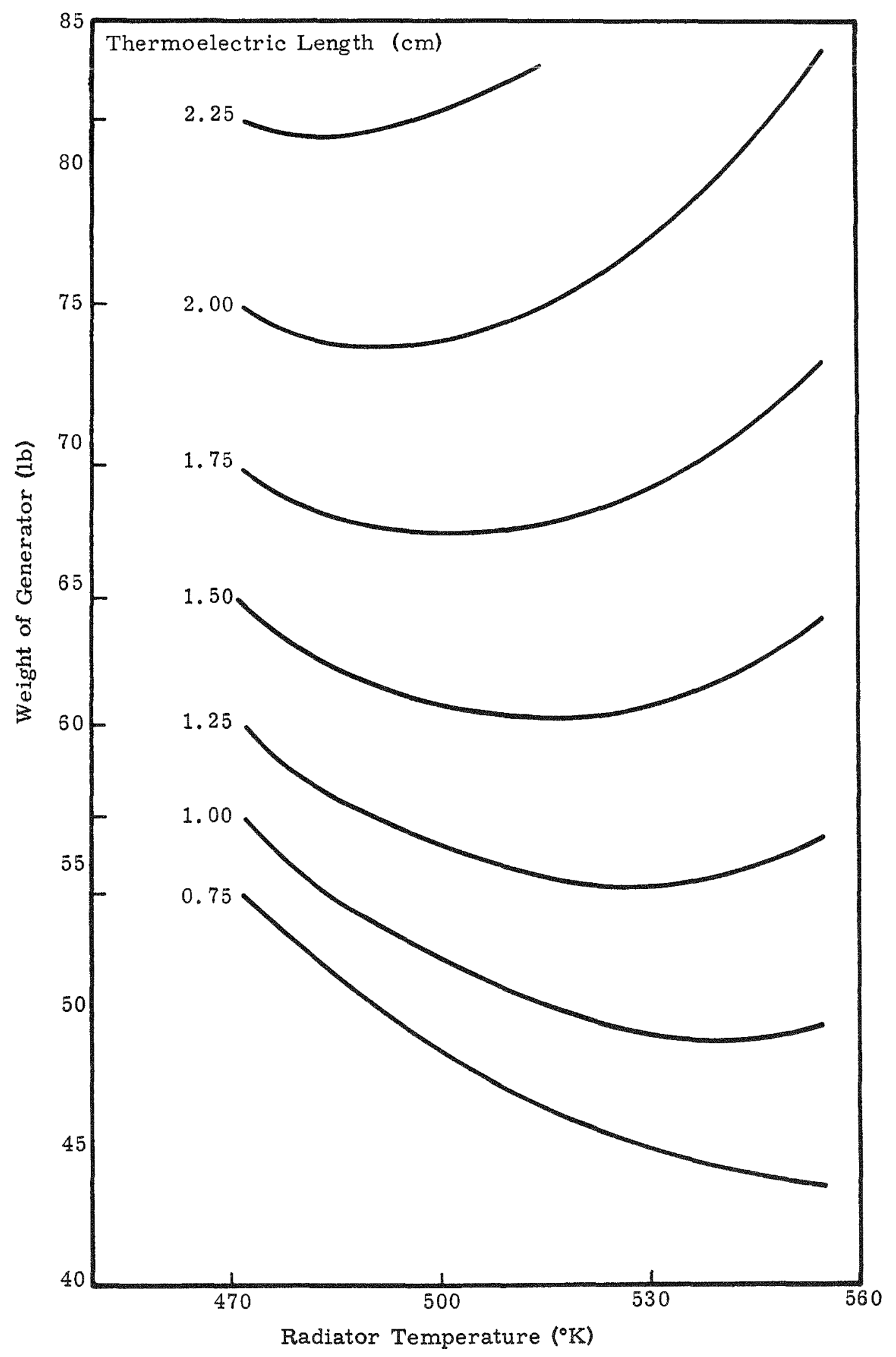

Fig. 73. Variation of Generator Weight with Radiator Temperature and Thermoelectric Length 
The centerline temperatures of a nickel-curium fuel slug for 10,15 , 20 and 25 to 1 nickel/curium weight ratios are given in Fig. 74. Also shown are the centerline temperatures for 10 and 20 to 1 iron/curium weight ratios.

Several assumptions were made in the calculation of the centerline temperature. The first of these is that the right circular cylinder with length equal to diameter can be approximated by a sphere of uniform surface temperature. The equation for the temperature at any point in a sphere was derived. The temperature is expressed as a temperature differential with the surface temperature assumed as zero for a reference point. Thus, the temperature differential between the surface and any point in the sphere is

$$
\Delta \mathrm{T}=\frac{\mathrm{S}}{6 \mathrm{k}}\left(\mathrm{R}^{2}-\mathrm{r}^{2}\right)
$$

At the center of the sphere, $r=0$, and the equation reduces to

$$
\Delta \mathrm{T}=\frac{\mathrm{SR}^{2}}{6 \mathrm{k}}
$$

where

$S=$ volumetric heat production rate

$R=$ outside radius of the sphere

$r=$ radius at any point inside the sphere

$k=$ thermal conductivity.

The thermal conductivity of pure nickel is $14 \mathrm{Btu} / \mathrm{hr}-\mathrm{ft}-{ }^{\circ} \mathrm{F}$ at $1200^{\circ} \mathrm{C}$. However, a value of $\mathrm{k}=10$ was used in the calculations because it is expected that the curium in the nickel will act as an impurity tending to reduce the thermal conductivity of the alloy。 For curium, $\mathrm{k}=7.5$. This is a room temperature measurement and the conductivity of a metal will usually decrease with increasing temperature. Also, less than $100 \%$ of theoretical density could have a detrimental effect on conductivity. Iron has a thermal conductivity of 40 to $45 \mathrm{Btu} / \mathrm{hr}-\mathrm{ft}-{ }^{\circ} \mathrm{F}$ at $1200^{\circ} \mathrm{C}$. For the same reasons given for nickel, the value of $\mathrm{k}$ was reduced to 30 。

The centerline temperature of a nickel-curium fuel pellet was considered where an alloy would not be found and the density or amount of compaction would be $90 \%$ of theoretical weight density. The temperature difference between the 2 conditions (alloy no alloy) was $5^{\circ} \mathrm{C}$ with the 


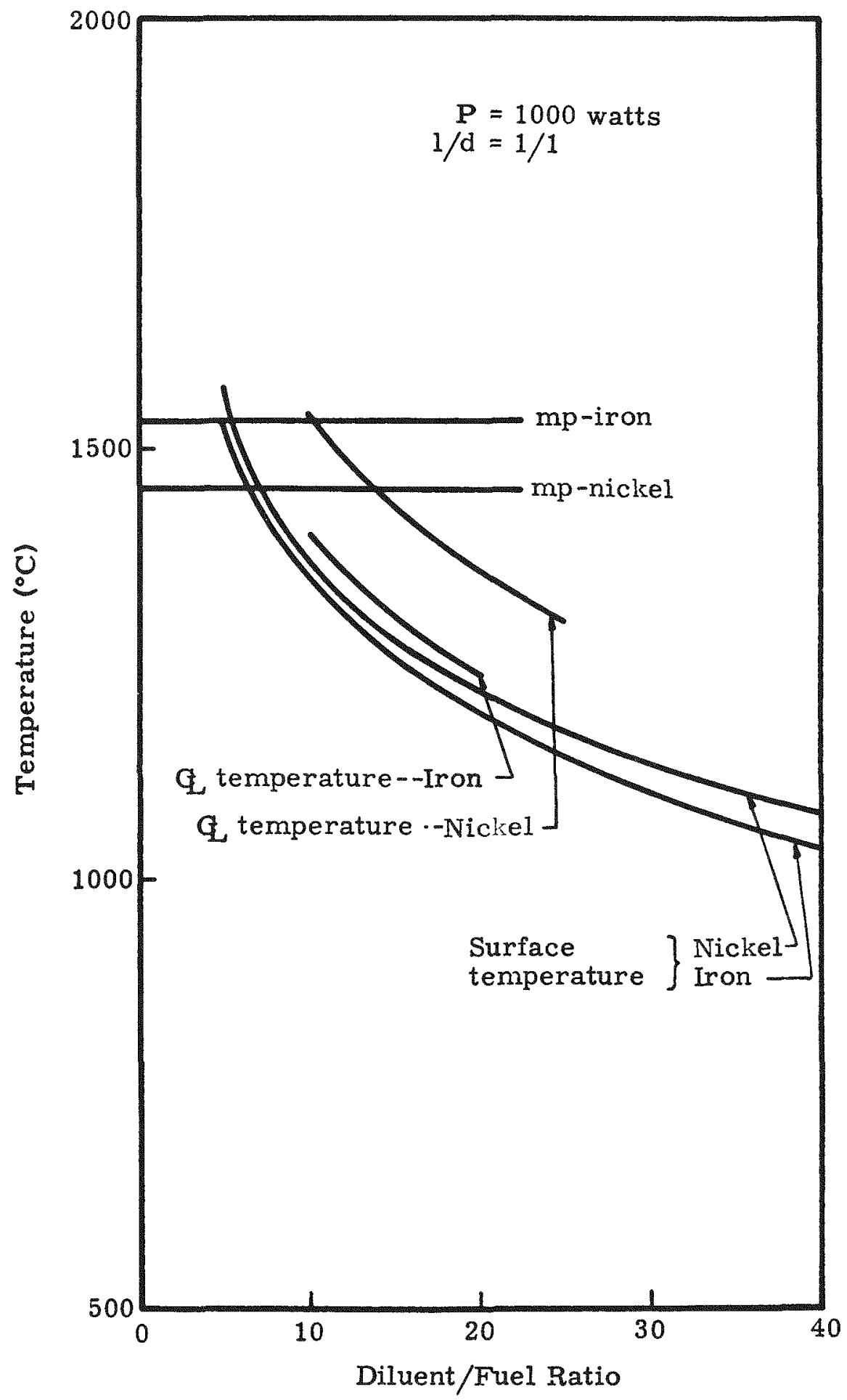

Fig. 74. Surface and Centerline Temperature of a Nickel-Curium or Iron-Curium Fuel Pellet 
lower temperature differential in the second case. A lower value of thermal conductivity in this case would probably more than offset this $5^{\circ} \mathrm{C}$ advantage.

A value of $100^{\circ} \mathrm{C}$ below the melting point of the matrix material has been assumed as a safe centerline temperature. Thus, the optimum diluent/fuel ratio for nickel is $20 / 1$. The value of $100^{\circ} \mathrm{C}$ is expected to compensate for depression of the melting point of nickel by curium and for small errors in the calculations. A diluent ratio of 10/1 for iron and curium would give a safety factor of $135^{\circ} \mathrm{C}$. Because a higher weight percentage of curium in iron is being considered, the increased safety factor is advisable as the curium may depress the melting point more severely as the percentage increases.

\section{B. GENERATOR AND COMPONENT DESIGN}

Generator design studies before October 1959 were based on the use of ablative material surrounding the heat source (fuel container). During the early portion of this quarter, general design philosophy required that the use of ablative material be avoided due to the requirement that the generating unit and its power source be capable of burnup upon re-entry. This necessitated revisions to the design optimization procedure.

In addition to the above, other considerations such as the necessity of a diluent to decrease surface temperatures of the heat source container commensurate with currently available container materials caused considerable effort to be spent on the final geometry of the unit.

The principal design effort concerned the radiative boundary spherical generator with biological shielding partly internal and partly external to the generator surface. This configuration is illustrated in Fig. 75. The fuel form in this configuration is an alloy of Curium-242 and nickel, with a ratio of 1 -curium atom to 20 nickel atoms. This form was selected on the basis of metallurgical and heat transfer studies performed undex Task 6; the results of these studies are as

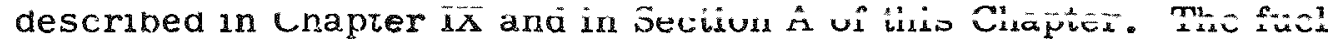
compound will be contained in a block of Inconel or Hastelloy $C_{;}$the choice between these was not made during this quarter. The fuel block is shown in schematic plan form in Fig. 76 .

The fuel block is mounted eccentrically along the vertical axis of the generator to increase the solid angle subtended by the heat dump at the fuel block and thus improve the effectiveness of the dump for power flattening. 


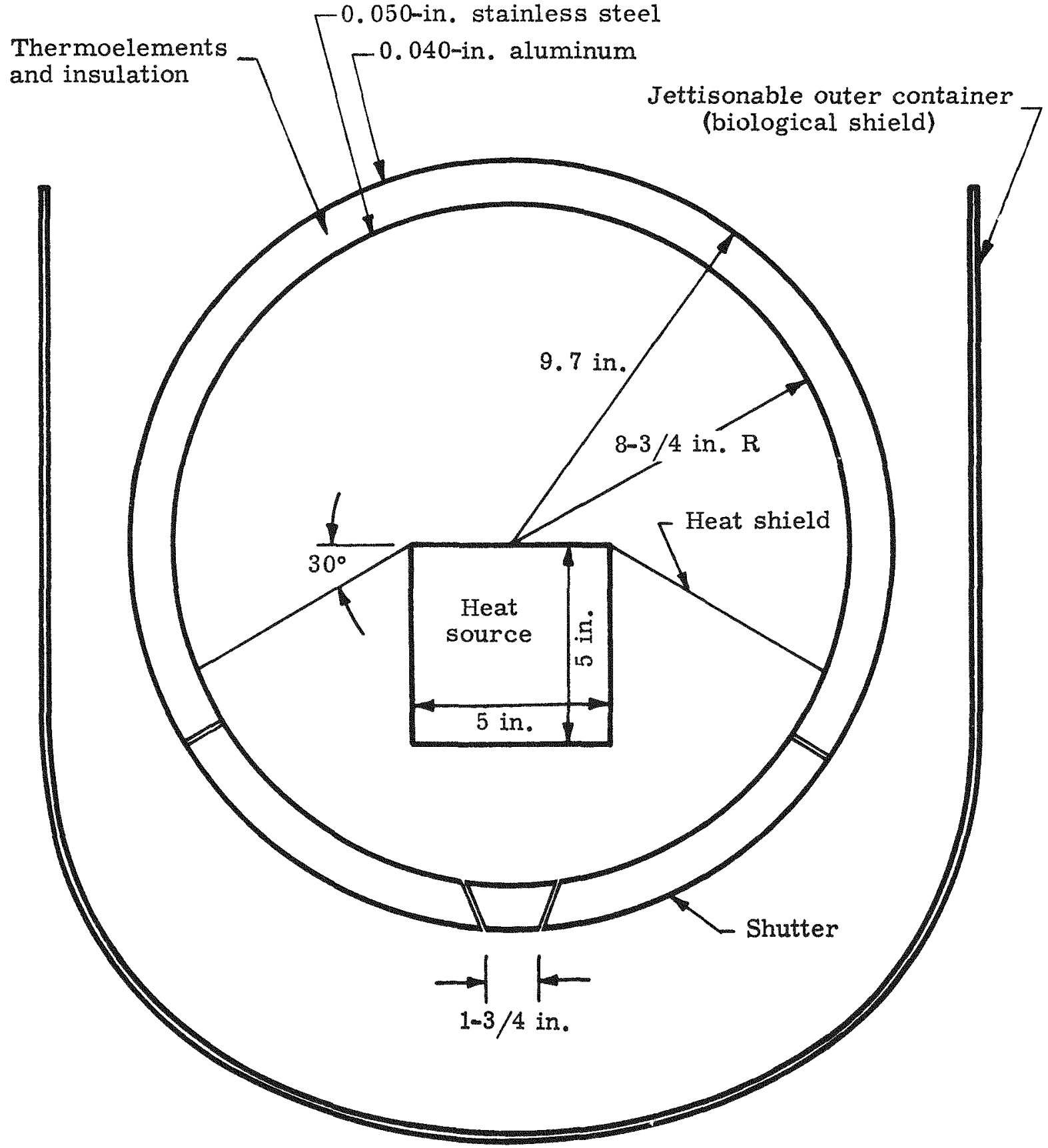

Heat source supports, cooling system and attachment to vehicle not shown

Fig. 75. Cross Section of Tentative Design for 100-Watt Generator 
The heat shield, a thin sheet of aluminum or stainless steel, permits the attainment of a more nearly uniform temperature distribution on the hot junction skin. This skin is of $0.050-i n c h$ stainless steel; the outer skin is 0.040 -inch aluminum, and the areas required for the radiator are coated with aluminum oxide. The thermoelements are lead telluride. While the analysis of the generator has been based on available data for Minnesota Mining and Manufacturing Company elements, other forms such as the extruded PbTe elements of Transitron Electronics Corporation may be adopted. The insulation is Min-K 1304. An extensive investigation of thermoelement hot shoes and hot junction mounting devices applicable to this program is de= scribed in Chapter VIII under Subtask 5.8 .

Power flattening is accomplished by moving shutters somewhat similar to those of the SNAP I-A generator. The shutters will be actuated by a device which uses thermal expansion of a metallic liquid-vapor system to move a rod linearly. In response to an invitation by The Martin Company to prepare design sketches of thermal-mechanical actuators, Air Research Corporation has submitted a promising design which is still being evaluated.

The use of radiator surface coatings with emissivities which depend on temperature was considered as a means of power flattening without moving parts. No suitable coating material has been found.

The external biological shielding container is assumed to be integral with the final stage of the launch vehicle and is not included in the generator weight.

In addition to the radiative spherical generator configuration, the following design types were examined:

(1) Radiative type, right cylindrical shape, with biological shield wholly internal.

(2) Radiative type, spherical shape, with biological shield wholly internal.

(3) Forced convection type with biological shield wholly external.

(4) Multiple 13-watt generators of the type designed under Subtask 5.8.

None of these types was sufficiently attractive with regard to weight and efficiency to warrant further study。 


\section{COMPONENT TESTS AND MEASUREMENTS}

The tentative program plan for Subtask 5.7, in effect at the beginning of this quarter, included the measurement of design data and the performance of dynamic environment tests on certain components and materials, especially thermoelements. Arrangements were initiated for measurement of the output voltage of mounted thermoelements during and after dynamic environment tests, including shock, acceleration and vibration. The effects of fast neutron irradiation on thermoelements was to be measured. The emissivity of several materials to be used as radiator coatings was to be determined as a function of temperature.

In order to establish data for the analysis of generators using other than $3 \mathrm{M} \mathrm{PbTe}$ thermoelements, a program was begun to measure the Seebeck coefficient $(\alpha)$ thermal conductivity $(k)$, and electrical resistivity $(\rho)$ of thermoelectric materials. The primary effort was in the development of instruments and procedures for the determination of $\alpha$ and $\mathrm{k}$, since $\rho$ is very easily measured.

Figure 77 shows the circuit used to measure $\alpha$ and $k$. Iron shoes 0.030 inch thick are soldered to each end of the element. Attached to these shoes are 30-gauge iron wires which are brought out to a d-c electrical source and voltage measuring device. A calibrated differential thermocouple assembly is bonded to the element with cement. Each of the thermocouple beads are in intimate thermal contact with, but electrically insulated from, the element. They are located within $1 / 64$ inch from the ends of the element and the lead wires are brought out along an isothermal line. Three other 20-gauge thermocouples are soldered to the element, one at each end and one in the center.

A small measured $d-c$ current is passed through the element, heating one end and cooling the others by the Peltier principle. When at equilibrium, the current (I), mean temperature $(T)$ and junction temperature difference $(\Delta T)$ are measured. The circuit is then opened, and the element $d-c$ output voltage $\left(\mathrm{e}_{\mathrm{oc}}\right)$ is measured by a trial and error reiterative method. The Seebeck coefficient is then

$$
\alpha=\frac{\mathrm{e}_{\mathrm{oc}}}{\Delta \mathrm{T}} \quad \text { microvolts } /{ }^{\circ} \mathrm{C}
$$

and

$$
k=\frac{\alpha I T}{\Delta T} \frac{\ell}{A}=\frac{e_{o c} I T}{(\Delta T)^{2}} \frac{\ell}{A} \frac{\text { watts }}{{ }^{\circ}-c m}
$$




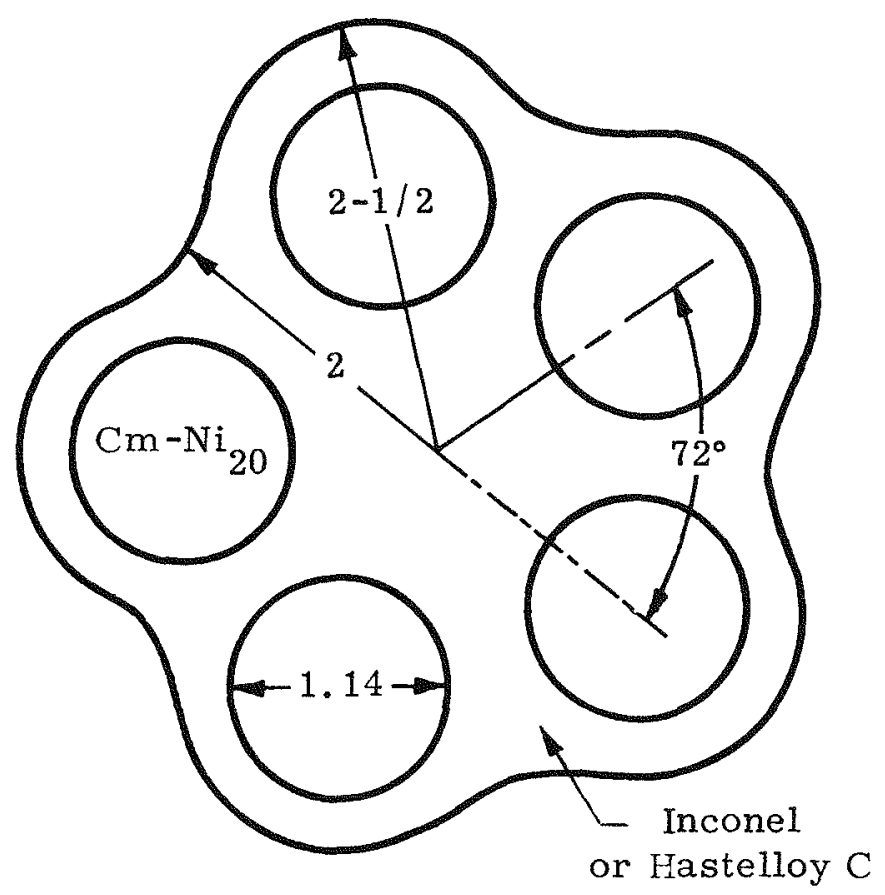

Fig. 76. Top View of Fuel Block (not to scale)

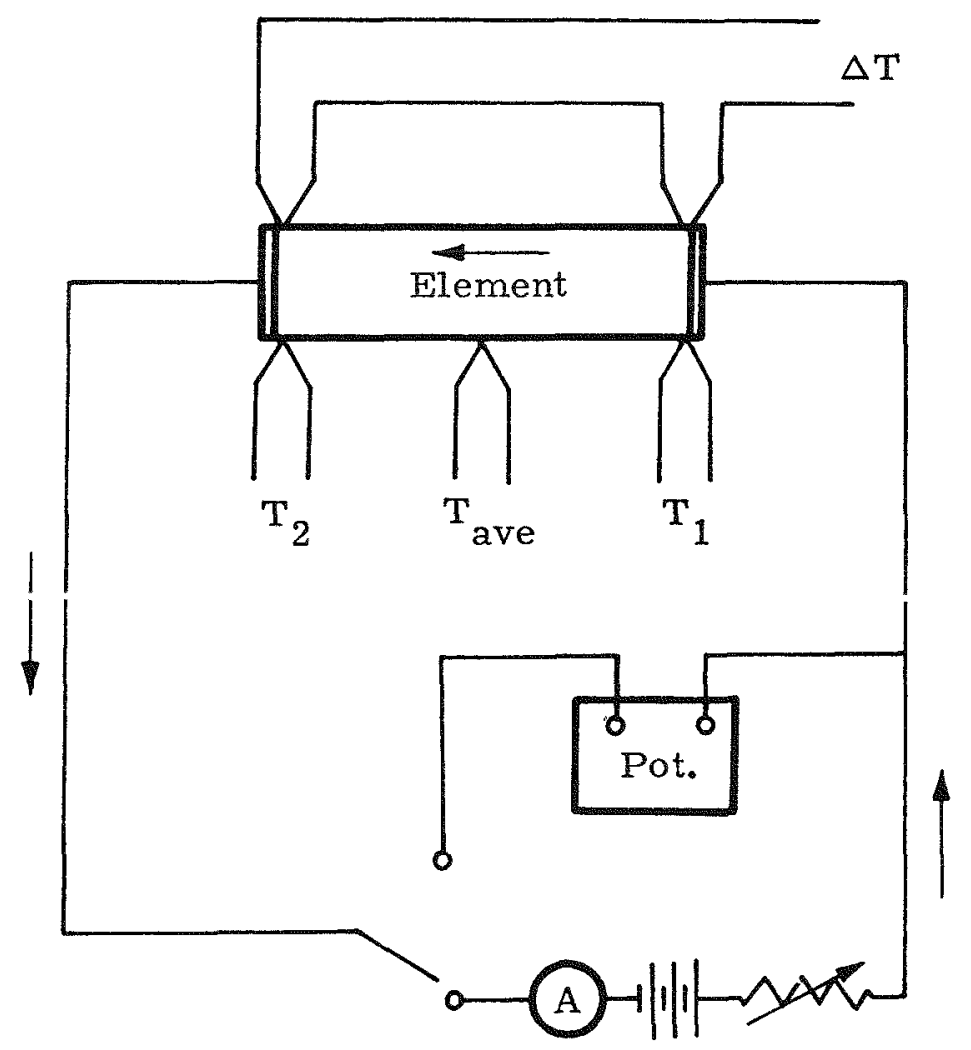

Fig. 77. Thermocouple Measurement Circuit 
where $\ell$ and $A$ are the element length and cross-sectional area, respectively。 $\mathrm{T}$ is approximately $10^{\circ} \mathrm{C}$.

The program plan was revised, and the testing efforts were terminated December 10,1959 。

\section{RADIATION SHIELDING DESIGN}

To permit safe handling of the generator on the ground as well as access to the launching vehicle during countdown, the nuclear radiation dose rate at 3 feet from the center of the generator must not exceed $60 \mathrm{mrem} /$ hour at any time before launch. The characteristics of radiation emitted by Curium-242 have been described in MND-1963 and MND-P-1972. In the case of the curium-nickel alloy fuel form. there are no $(\alpha, n)$ reactions. The gamma emission is so weak that the shielding requirements are established entirely by spontaneous fission neutrons.

Shielding thicknesses were calculated by the Albert-Welton point kernel method and by the moments method. The methods of analysis are described in detail in Appendix $D$. The necessary thickness of a saturated aqueous solution of boric acid is 9.75 inch. Solutions of lithium salts are equally good shielding materials. Since the shielding requirement, to some extent, is a constraint on generator size and weight, accurate estimates are quite important. Most of the calculations were performed for the radiative boundary generator with internal shielding, before this quarter. The other configurations require nearly identical shielding thicknesses.

The shielding liquid must be removed before launch in order to save weight. Means of accomplishing this are being developed.

An investiation was made of the contribution by decay products of Curium-242 to total dose rates. The results are listed in Table 10 .

The amount of Curium-242 decay products present was plotted as a function of time. The curves were calculated with the assumption that none of the daughters are present at the start $(t=0)$. If any of the daughters are present at the start similar curves for each of these isotopes must be calculated and the ordinates added to the curves first mentioned.

Dose rates were calculated on a per gram basis and, when these values are multiplied by the fuel weight, the contribution to the total dose rate becomes negligible. With the exception of the 
gammas from $\mathrm{Bi}-214$ (one of the daughters of $\mathrm{Ra}-2260$ the energies are less than $1 \mathrm{Mev}$. Since more than 30 years are required for microgram quantities of $\mathrm{Ra}-226$ to accumulate, these high energy gammas are negligible.

TABLE 10

Maximum Dose Rates From Curium Decay Products During Operational

Life of the Unit

\begin{tabular}{lll} 
Isotope & $\begin{array}{c}\text { Gamma Dose Rate per Gram at } 1 \text { Meter } \\
\left.(\mathrm{mr} / \mathrm{hr}) \text { (From } \gamma^{\prime} \mathrm{s} 0.04 \mathrm{Mev}\right)\end{array}$ & $\begin{array}{c}\text { meem/hr per Gram at } 1 \\
\text { Meter for Neutrons }\end{array}$ \\
\cline { 3 - 3 } & 0.0067 & $5 \times 10^{-3}$ \\
$\mathrm{U}-234$ & $6.64 \times 10^{-7}$ & $8.05 \times 10^{-7}$ \\
$\mathrm{Th}-230$ & No gammas over $0.4 \mathrm{Mev}$ & $1.1 \times 10^{-9}$ \\
$\mathrm{Ra}-226$ & $1000 \mathrm{mr} / \mathrm{hr} *$ & $\begin{array}{l}\text { No spontaneous } \\
\text { fissioning }\end{array}$
\end{tabular}

*Includes decay products of Ra-226

\section{E. HAZARDS EVALUATION}

Evaluations of hazards associated with the production and use of Curium-242 have been reported in MND1963. A great portion of the hazards evaluations made under Task 1 and Task 2 is applicable to the present program.

The hazards analysis for this program is based on the philosophy that safe containment of the fuel must be assured under all credible circumstances and for as long as it may present any radiological hazard. The mission for inis generator nas nor been $11 \mathrm{rmiy}$ established, but it is assumed that it will be used in a satellite, lunar mission or inter. planetary mission. Consequently, containment must be assured in case of burning of the vehicle on the launch pad, destruction shortly after liftoff, or an abort in which the vehicle impacts with the earth's surface at high velocity. During re-entry of the orbiting satellite, the fuel is to be burned up in the upper atmosphere.

The problem of safe containment of the fuel at elevated temperatures with helium pressure buildup resulting from alpha emission was examined. Some of the results are also applicable to the 13 -watt generator (Subtask $5.8)$. 
The alpha particles emitted by the Curium-242 will acquire orbital electrons and become helium atoms. Allowance must be made for this accumulation of helium, so the fuel container should contain a void equal to $20 \%$ of the volume of the fuel and the vessel must be strong enough to sustain the resulting internal pressure. The quantity of helium at any time after encapsulation can be calculated by the equation

$$
\mathrm{N}=\mathrm{N}_{0}\left(1-\mathrm{e}^{-\lambda t}\right)
$$

where

$$
\begin{aligned}
N= & \text { moles of helium } \\
N_{0}= & \text { moles of } \mathrm{Cm}-242 \text { at encapsulation } \\
\lambda= & \text { disintegration constant of } \mathrm{Cm}-242 \\
& 4.25 \times 10^{-3} \text { day }^{-1} \\
t= & \text { time after encapsulation in days. }
\end{aligned}
$$

Having the quantity of helium at any time, the pressure can be calculated for any given temperature and volume.

$$
P=\frac{N R T}{V}
$$

where

$$
\begin{aligned}
& \mathrm{P}=\text { pressure in atmospheres } \\
& \mathrm{N}=\text { moles of helium } \\
& \mathrm{R}=\text { universal gas constant }=0.082 \mathrm{~atm} \text { liters } / \text { mole }{ }^{\circ} \mathrm{K} \\
& \mathrm{V}=\text { volume of void in liters. }
\end{aligned}
$$

Calculations of the pressures to be expected were made on the following assumptions: the volume of fuel is $142 \mathrm{cc}$ and a void of $28.4 \mathrm{cc}$ is provided: the mass of $\mathrm{Cm}-242$ is $62 \mathrm{gm}$, equivalent to 0.257 mole; and the temperature of the void is $1273^{\circ} \mathrm{K}\left(1000^{\circ} \mathrm{C}\right)$. It was found that the pressure at the start of the mission, 60 days after encapsulation, is 3140 psi. At the end of the mission, 242 days after encapsulation, it is $8940 \mathrm{psi}$. If the temperature decreases, the pressure will be reduced proportionately. After 1250 days, the pressure would be about 13,740 psi if the temperature were maintained at 
$1273^{\circ} \mathrm{K}$. However, the temperature will have fallen almost to ambient at this time. For a temperature of $293^{\circ} \mathrm{K}\left(20^{\circ} \mathrm{C}\right)$, the pressure would be 3160 psi. A more precise calculation of the pressure will be made when the temperature-time profile is known more definitely. 
VIII. SUBTASK 5.8--CONCEPTUAL DESIGN OF 13-WATT THERMOELECTRIC GENERATOR *

The objective of this program is to design a reliable, lightweight, ef ficient and versatile power supply for instruments and transmitters aboard space probes. In order to make the supply rugged, nearly free of moving parts, and essentially independent of its environment, an isotope-fueled thermoelectric generator will be used. Stringent requirements on nuclear radiation dictated Curium-242, which is a very weak emitter of neutrons and gamma rays, as the isotope fuel.

The originally proposed application of the generator was a six-month mission in nearby space; e.g., a circumlunar probe. The generator was to deliver 13 watts of continuous unregulated electrical power throughout the six-month period; it was to weigh not over 15 pounds exclusive of voltage regulators and DC-DC converters. The generator was to operate normally after subjection to the following environmental conditions based on the Vega specifications:

(1) Acceleration--Rotation about any axis of the generator at 700 rpm.

(2) Vibration--15 g of white Gaussian noise from 20 to $2000 \mathrm{cps}$ for 10 minutes in each of three mutually perpendicular planes.

(3) Shock --4 axial shocks of $25 \mathrm{~g}$, each lasting 20 milliseconds with a 1-millisecond rise time.

With the cancellation of the Vega program, these quantitative conditions no longer apply but are illustrative of a typical environment.

All of the work performed thus far has been directed toward satisfaction of these criteria. In December 1959, Jet Propulsion Laboratory recommended to the National Aeronautics and Space Administration that the application of the generator be to a lunar impact vehicle. The payload is to impact at a random altitude on the moon at a maximum design velocity of $500 \mathrm{ft} / \mathrm{sec}$. By means of crushable structure around the generator, the acceleration sustained by the power unit is approximately $400 \mathrm{~g}$. After impact, the generator is to deliver 12 watts of unregulated electrical power for at least 30 days and preferably for 60 days. The weight limitation in increased to 18 pounds. The original requirement that the operational photon flux was not to exceed 7 photons / sq $\mathrm{cm}-\mathrm{sec}$ above $100 \mathrm{kev}$ at a point $10 \mathrm{~cm}$ from the generator surface was altered to 1 photon/sq cm-sec from 0.4 to $3 \mathrm{Mev}$, and 0.5 
photon/sq $\mathrm{cm}-\mathrm{sec}$ from 3 to $10 \mathrm{Mev}$, at 5 meters from the generator on a circular area of $100 \mathrm{sq} \mathrm{cm}$. A revision of this program to meet the altered conditions has been proposed to the AEC.

Prior to the period covered by this report, several general configuration types were proposed; analysis and design studies were to provide the basis for selection of the configuration, optimized with respect to weight and overall efficiency, which would be the subject of more detailed investigation. The proposed generator types were:

(1) Several units, each modeled closely on the SNAP III design with minor modifications required by the use of Curium-242 instead of Polonium-210. The hot shoes of the thermoelements are directly attached to the fuel container.

(2) A single enlarged SNAP III-type generator, still with conduction as the means of heat transfer from the fuel to the thermoelements.

(3) A generator in which heat is radiated from the fuel container to a spherical skin to which the hot shoes are attached. The cold junctions of the thermoelements are in contact with a concentric external spherical radiator. As a modification of this design, in order to improve fabricability without strongly perturbing the isotropy of heat flow, the spherical surfaces are replaced by two 14-sided polyhedrons.

(4) A radiative-type generator with the thermoelements located between two right-circular cylinders which are coaxial with the fuel container. The lateral or curved surfaces are used for thermoelements; the ends are available for the heat dump and attachment to the payload.

Analysis of the first of these models began in September 1959 and continued into October. For completeness, all of this work will be described in the following section.

\section{A. HEAT TRANSFER AND THERMOELECTRIC ANALYSIS*}

The analyses of a spherical radiative-type, a multiple SNAP III-type, a scaled-up SNAP III-type and a cylindrical radiative-type generator are reviewed below. These designs incorporate the latest thermoelectric data using sodium and lead iodide doped, unsegmented lead telluride ele- 
ments. Since the cylindrical configuration was selected as the final design, additional investigations were made of this generator. These include the use of cobalt silicide elements and the inclusion of a heat dump mechanism.

\section{Multiple SNAP III-Type Unit}

Analysis. Using Singer's ${ }^{1}$ equation (Appendix E) to determine the surface temperature of a SNAP III-type (1G5) unit operating in space, it was found that the surface temperature will be approximately 50 degrees lower than that obtained in the laboratory for vacuum conditions radiating to $70^{\circ} \mathrm{F}$ ambient. Laboratory data indicate the maximum electrical power for each unit is 2.88 watts with a $1000^{\circ} \mathrm{F}$ hot junction temperature. With these results and the design requirement of 13 watts electrical power, it becomes necessary to use 5 units. The resulting weight is approximately 20 pounds, 5 pounds over the maximum permissible weight of 15 pounds. Although the increased overall efficiency that will result from space operation may enable four units to be used, the total weight is still over the maximum weight. On the other hand, each unit will partially shield the others from radiating heat into space, lowering the thermal efficiency. Thus, the design is not feasible unless the power output per generator can be increased by using part of the satellite structure for heat dump purposes; or fins might be used to reduce the cold junction temperature, which would accomplish the same purpose. To meet the specifications by other techniques requires a redesign of this generator.

\section{Summary of Results--1G5 Unit}

Overall efficiency

Hot junction temperature

Cold junction temperature

Power

Electrical output
$4.96 \%$

$1000^{\circ} \mathrm{F}$

$215^{\circ} \mathbf{F}$

58 thermal watts

2.88 watts

NOTE: There is some question as to whether the data used were obtained in vacuum conditions or in air at atmospheric pressure. The data as discussed here are treated so as to result in a low generator weight. The actual system may be heavier to give the required power, but will not be lighter. 


\section{Spherical Radiative-Type Unit}

Analysis. The basis for this design is the attainment of maximum overall efficiency in a thermoelectric generator. This overall efficiency depends on the thermal and thermoelectric efficiencies, which are in turn dependent on the heat flow through the insulation and the hot and cold junction temperatures. Figure 78 indicates that, as the cold junction temperature is lowered (that is, the temperature difference between the hot and cold junction increases for a constant hot junction temperature), the thermoelectric efficiency increases. However, as shown in Fig. 79, increasing the temperature difference also increases the heat loss through the insulation, thus lowering the thermal efficiency. Therefore, an optimum overall efficiency exists which can be obtained by an iterative technique.

Figure 80 is a sketch of this type unit. The following assumptions were made in this design:

(1) The temperature drop through the hot and cold junction shoes is small in comparison to the temperature drop through the thermoelectric elements.

(2) No heat is lost except that which passes through the insulation.

This design includes the following parameters:

(1) The hot junction temperature is $1000^{\circ} \mathrm{F}$.

(2) Insulation is 1.25-inch Min-K.

(3) The length of the thermoelements is 0.75 inch.

(4) The fuel form is an impacted powder 1 part (by weight) $\mathrm{Cm}$ 242 to 6.5 parts $\mathrm{Pt}$. Redesign for an alloy of 1 part $\mathrm{Cm}-252$ to 20 parts $\mathrm{Ni}$ is in progress.

(5) The unit internal pressure is 0.5 atmosphere (air).

(6) Length-to-diameter ratio of the fuel source is 1.5 .

(7) The inner and outer shell are aluminum*

(8) A 0.5 -inch molybdenum shield is placed around the heat source**.

\footnotetext{
*The inner shell will have to be made of high temperature material. Since this has not been chosen, aluminum was assumed for weight calculation purposes.

* Substitution of Hastelloy $\mathrm{C}$ will be considered later.
} 


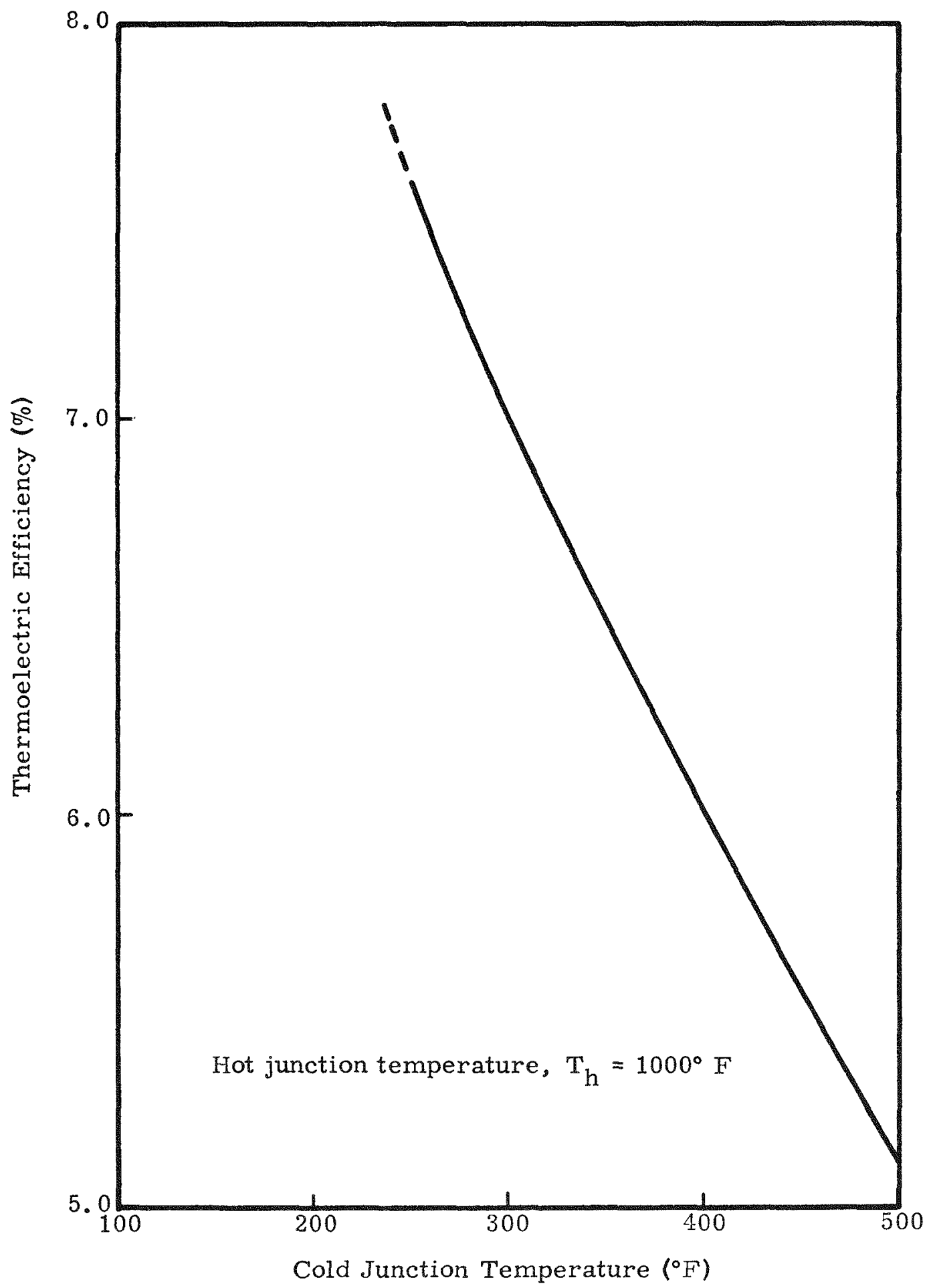

Fig. 78. Thermoelectric Efficiency Versus Cold Junction Temperature for $\mathrm{PbTe}$ elements 


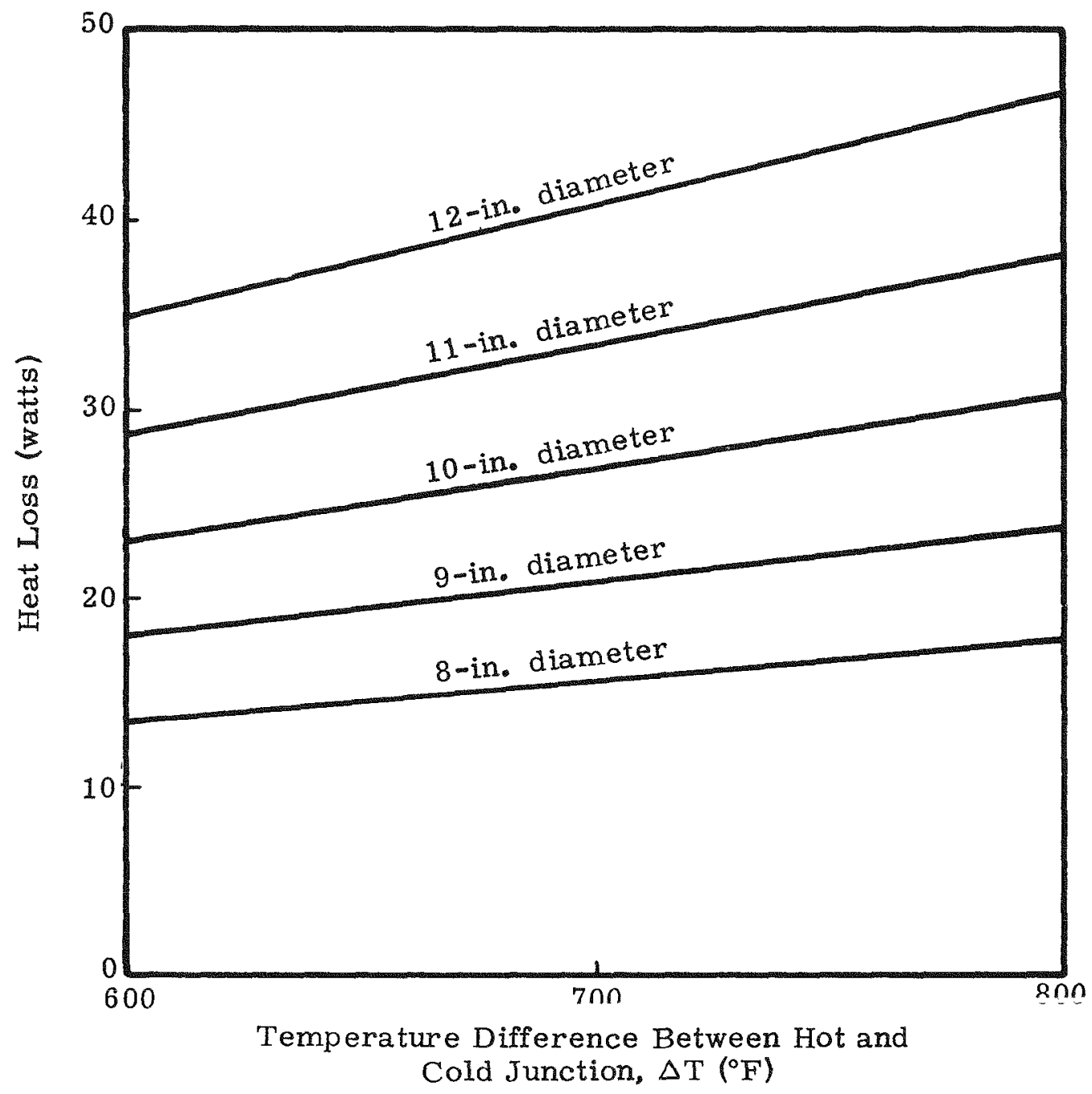

Fig. 79. Heat Loss for Radiative-Type Spherical Unit 


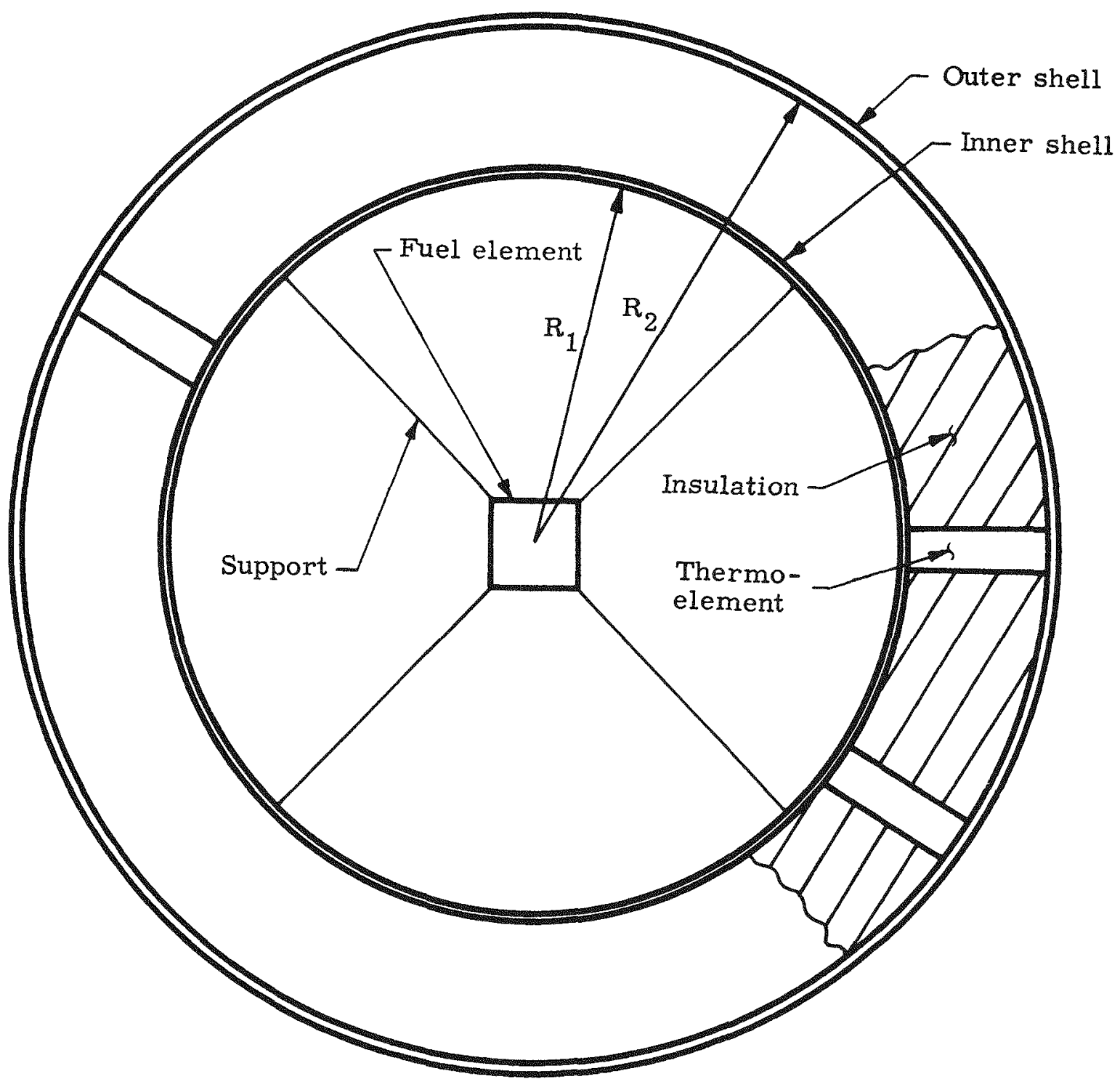

Fig. 80. Radiative Unit 
Procedure. With an assumed minimum overall efficiency of $4 \%$, the isotope loading required to produce 13 electrical watts was established. With this power requirement, a length-to-diameter ratio of 1.5 and the known power density of Curium-242, the fuel element was sized. This power requirement was also used to determine the outer surface area of a sphere that would dissipate this heat and give a practical cold junction temperature.

Since generator heat loss is an important factor in the thermal efficiency, curves of heat loss versus temperature difference were constructed using the usual equation for radial heat flow through a sphere. These data are presented in Fig。79.

For the system to operate for six months, a hot junction temperature of $1.000^{\circ} \mathrm{F}$ (which is a practical temperature for lead telluride thermoelements) was chosen. With this temperature and several assumed cold junction temperatures, the thermoelectric efficiency curve (Fig.78) was constructed.

It should be noted that, in order to operate for a 6 -month period, some means of temperature control is necessary to compensate for isotope decay. An investigation of various control techniques was not done as a part of the work for this design.

To begin the calculation, an overall efficiency was assumed and a cold junction temperature determined by employing Singer's equation for satellite temperatures. This temperature fixed the thermoelectric and thermal efficiencies. If the calculated overall efficiency did not agree with the assumed overall value, another efficiency was chosen and the entire procedure was repeated. This trial and error process was continued until the assumed overall efficiency and the calculated overall efficiency were in agreement, thus giving a generator design optimized on the basis of thermoelectric efficiency* Several of these calculations were then made to establish a generator design based upon overall ef ficiency. The weight of each unit was then determined by combining the weight of all the components and, provided the weight was under the 15pound maximum, a practical design was assumed established for that particular diameter.

Summary of results. Results of the radiative-type unit investigation are shown graphically in Figs. 81 and 82 . Figure 81 , efficiency versus generator diameter, shows that for a fixed thickness of insulation $(1.25$ in.) the overall efficiency peaks at a 9.25 -inch diameter unit.

*The equations used for this calculation were derived on the basis of optimized thermoelectric efficiency. 


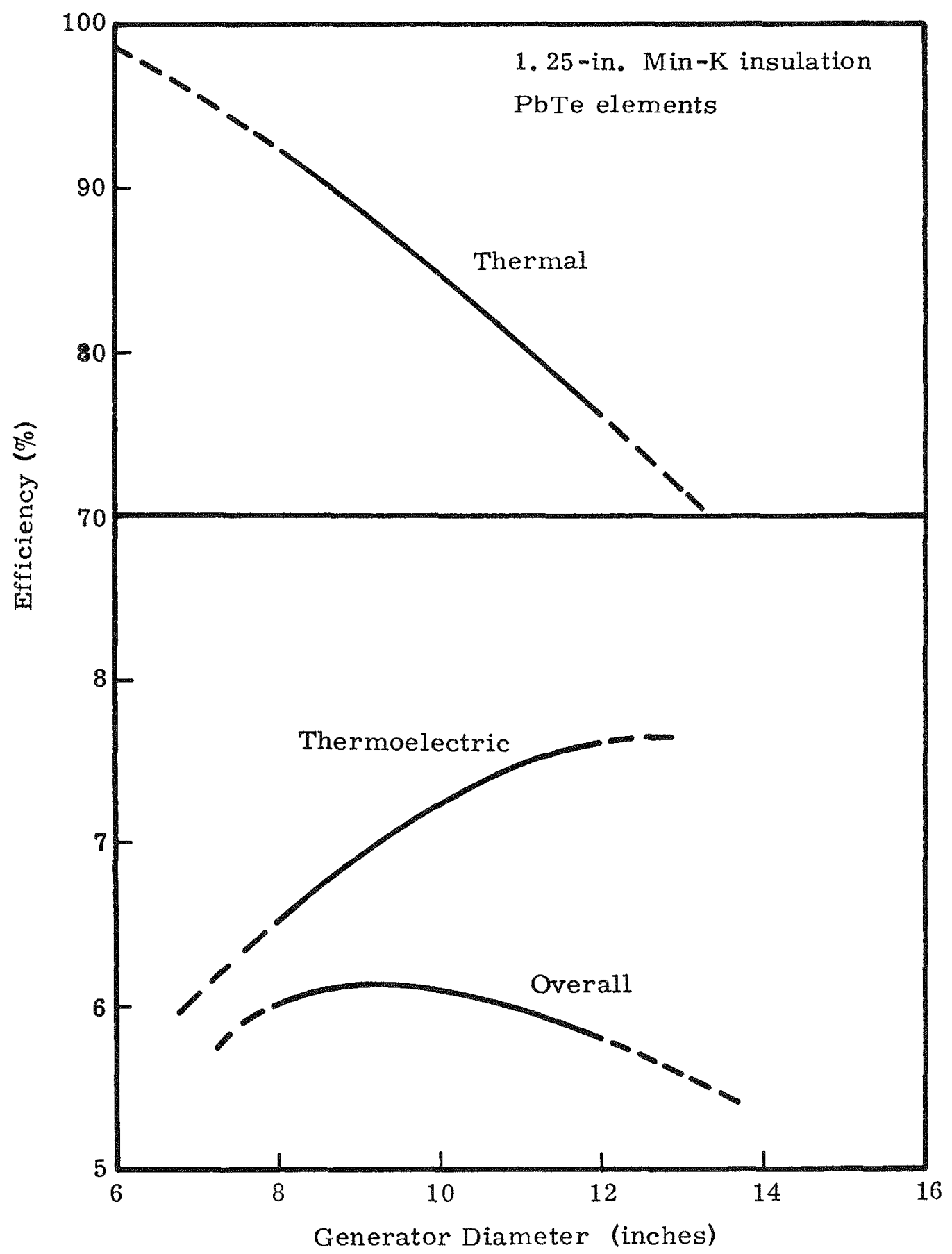

Fig. 81. Efficiency Versus Generator Diameter, Radiative-Type Unit 


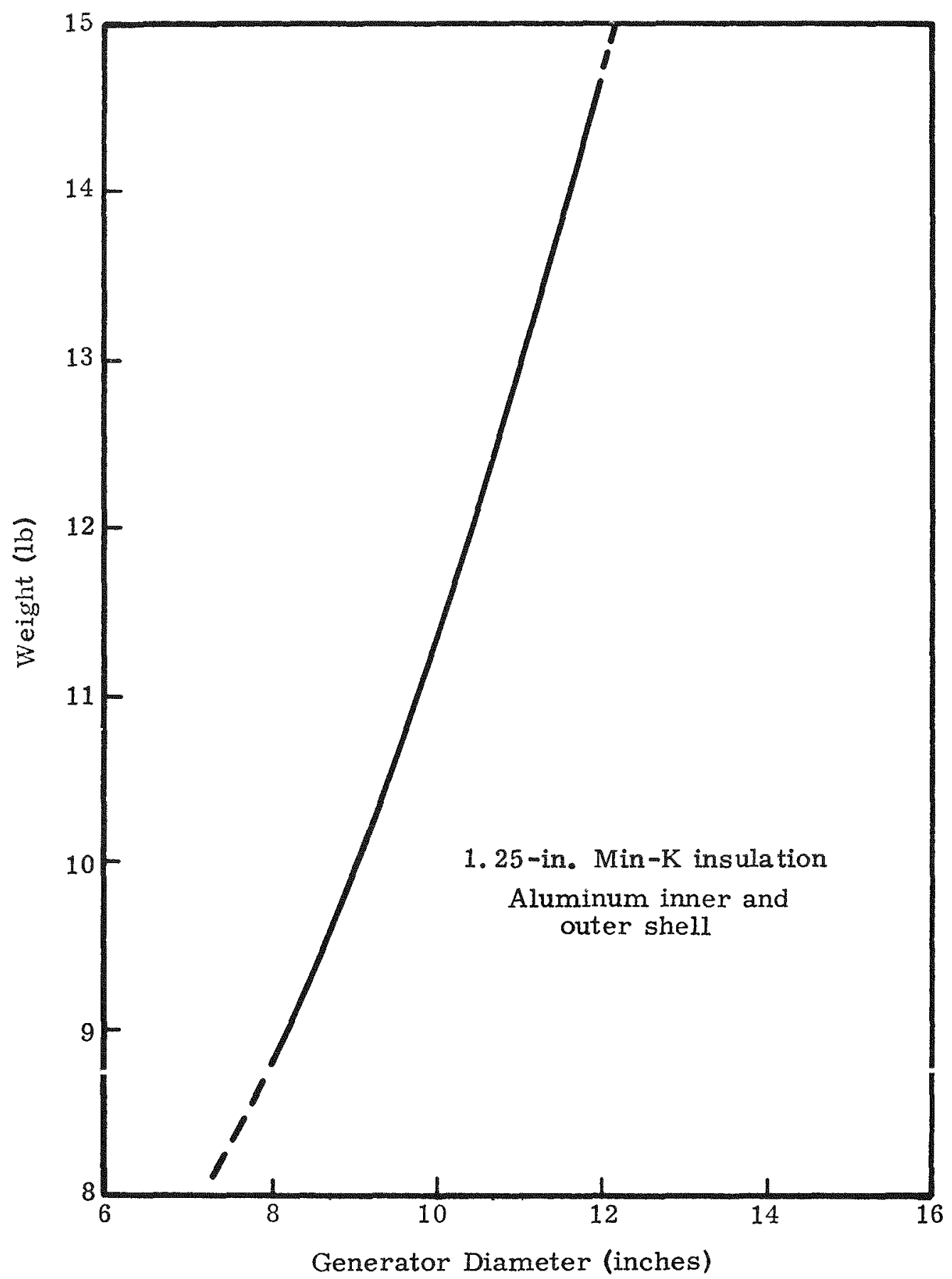

Fig. 82. Generator Weight Versus Diameter, Radiative-Type Unit 
Figure 82 is a graph of generator weight versus diameter. This plot shows that the weight increases very rapidly with increasing diameter, but the 15-pound maximum has not been exceeded for the size units investigated.

Table 11 lists specifications for generator diameters of $8,9,10$ and 12 inches.

TABLE 11

Radiative-Type Generator Specifications

Unit Diameter

8 Inches 9 Inches 10 Inches 12 Inches

Efficiency (\%)

Thermoelectric

Thermal

Overall

$\begin{array}{cccr}6.51 & 6.95 & 7.22 & 7.6 \\ 92.2 & 88.21 & 84.3 & 76.0 \\ 6.0 & 6.13 & 6.1 & 5.8\end{array}$

Temperature $\left({ }^{\circ} \mathrm{F}\right)$

Hot junction

1000

1000

1000

280

305

235

Heat loss (watts)

17

25

33

54

Insulation thickness (in.)

1.25

1.25

1.25

1.25

Weight (pounds)

Molybdenum fuel containes and fuel

2.41

2.41

2.41

2.41

Outer shell aluminum

(35 mils)

0.69

1.03

1.27

1.82

Inner shell aluminum

(35 mils)

0.32

0.54

0.71

1.14

Thermoelectrics

1.52

1.30

1.22

1.10

Thermal insulation

$\frac{2.09}{7.03}$

3.50

5.28

Subtotal

8.04

9.11

11.75

Structures

Total

$\begin{array}{llll}1.76 & \frac{2.06}{8.79} & \frac{2.28}{10.05} & \frac{2.95}{11.39}\end{array}$


TABLE 11 (continued)

Unit Diameter

8 Inches 9 Inches 10 Inches 12 Inches

Thermoelectric elements

Output voltage

Number of pairs

3

29

0.109

0.131

0.75

0.75

28
3

27

26

Cross-sectional area (sq in.)

$\mathrm{N}$ elements

$P$ elements

Length (in.)

NOTE: All $\mathrm{P}$ elements doped with $1.0 \%$ sodium and all $\mathrm{N}$ elements doped with $0.03 \%$ lead iodide.

3. Scaled-Up Model

Analysis. The basis for this design and the assumptions used are the same as for the radiative unit, but the design parameters are as follows:

(1) The hot junction temperature is $1000^{\circ} \mathrm{F}$.

(2) The length of the thermoelements is one inch.

(3) Unit internal pressure is 0.5 atmosphere (air).

(4) Iength-to-diamater ratin of the fuel source is 1.5 .

(5) Outer shell is aluminum.

(6) A 0.5 -inch molybdenum shield is located around the heat source.

(7) All voids in the generator are filled with insulation. Figure 83 is a sketch of this unit.

Procedure. The procedure for this type unit is the same as for the radiative-type unit with the exception of the determination of heat flow through the insulation. 


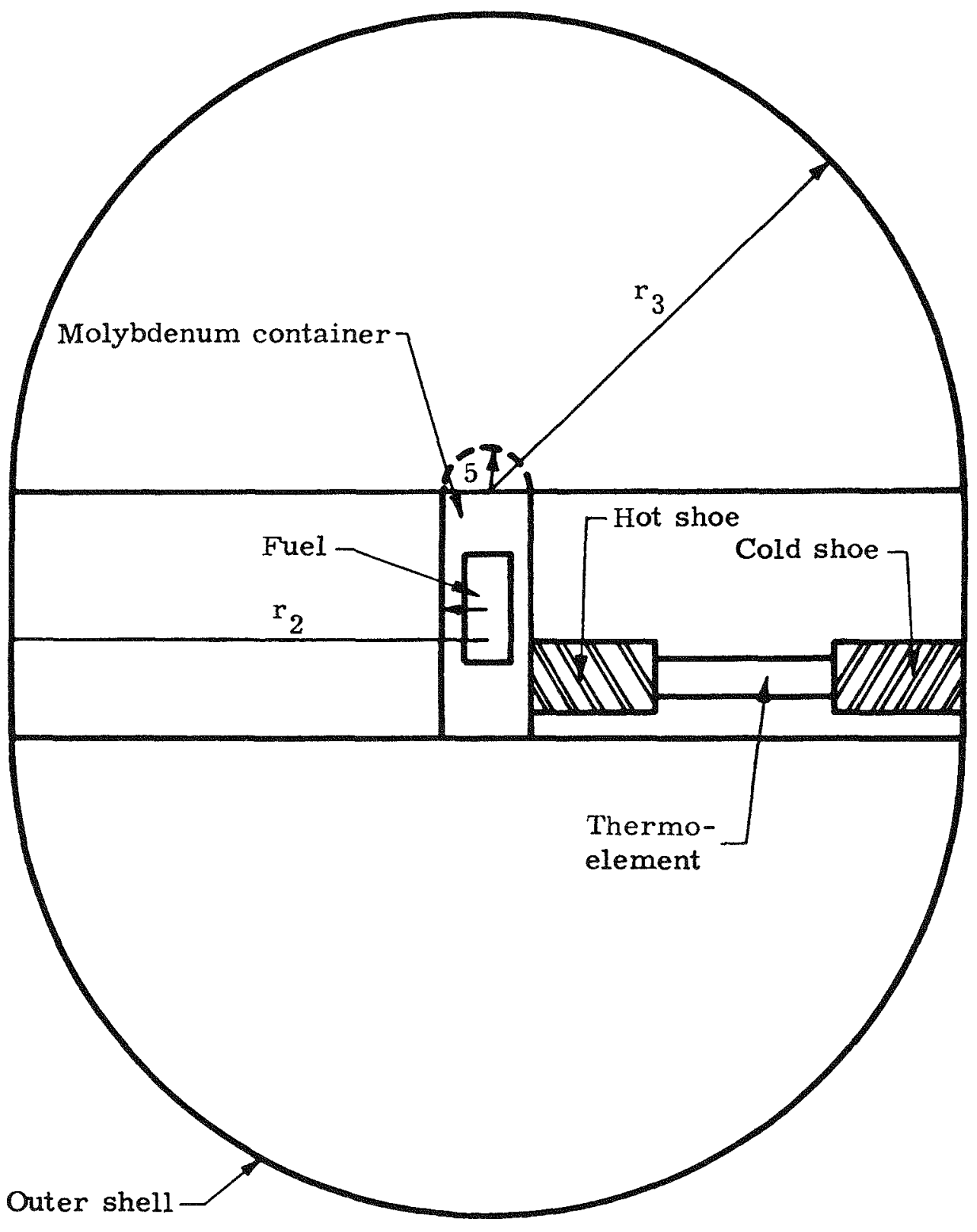

NOTE: All voids filled with insulation

Fig. 83. Scaled-Up Unit 
This heat loss was determined using a combination of heat flow through a sphere and heat flow in a hollow cylinder. Figure 84 is a plot of temperature difference versus heat loss.

Using this curve and proceeding as discussed previously, the thermal efficiency, thermoelectric efficiency, overall efficiency and weight of the unit was determined for several sizes.

Summary of results. Results for the scaled-up model investigation are shown in Fig. 85 . The thermal efficiencies vary less than $1 \%$ over the range investigated; therefore, the thermoelectric and overall efficiencies follow approximately the same curve. These efficiencies increase rapidly as the unit diameter is increased. However, a small increase in diameter results in a significant weight increase. Figure 85 shows that a unit diameter of over 9-1/2 inches will yield a weight greater than the 15-pound minimum. Therefore, units with diameters greater than 9-1/2 inches are not feasible for this application.

Table 12 lists specifications for generator diameters of 6,8 and 10 inches.

TABLE 12

Scaled-Up Model Generator Specifications

Unit Diameter

6 Inches 8 Inches 10 Inches

Efficiency $(\%)$

Thermoelectric

Thermal

Overall

Temperature $\left({ }^{\circ} \mathrm{F}\right)$

Ust junstion

Cold junction

Heat loss (watts)

Weight (pounds)

Heat source and container

Outer shell

$\begin{array}{lll}5.56 & 6.83 & 7.65 \\ 98.8 & 98.5 & 98.3 \\ 5.51 & 6.73 & 7.50\end{array}$

$\begin{array}{lll}10 n n & 10 n n & \begin{array}{l}1 n n n \\ 430\end{array} \\ 3 & 310 & 230 \\ & 3 & 3 \\ & & \\ 2.41 & 2.41 & 2.41 \\ 0.57 & 0.96 & 1.46\end{array}$




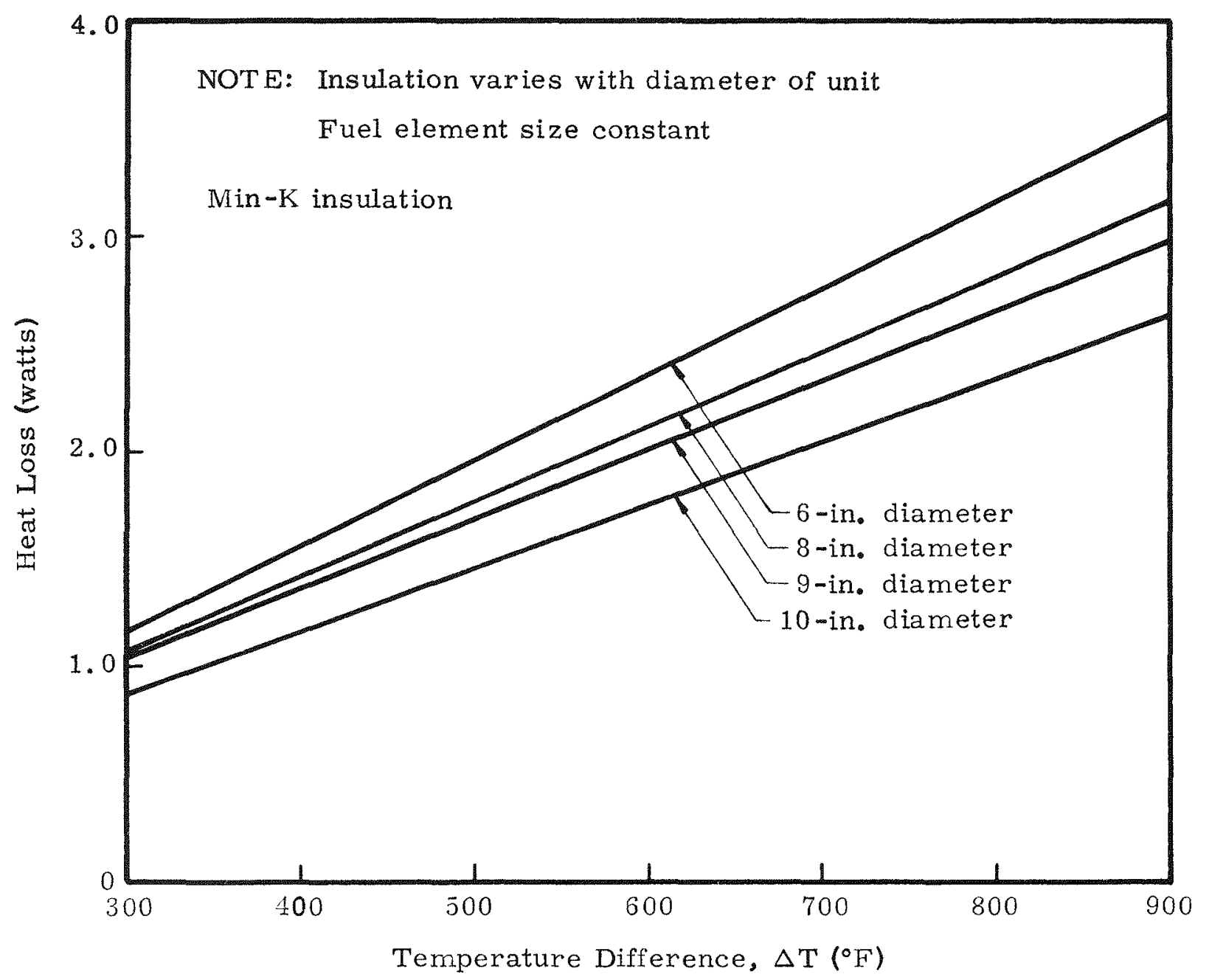

Fig. 84. Temperature Difference Versus Heat Loss, Scaled-Up Model 


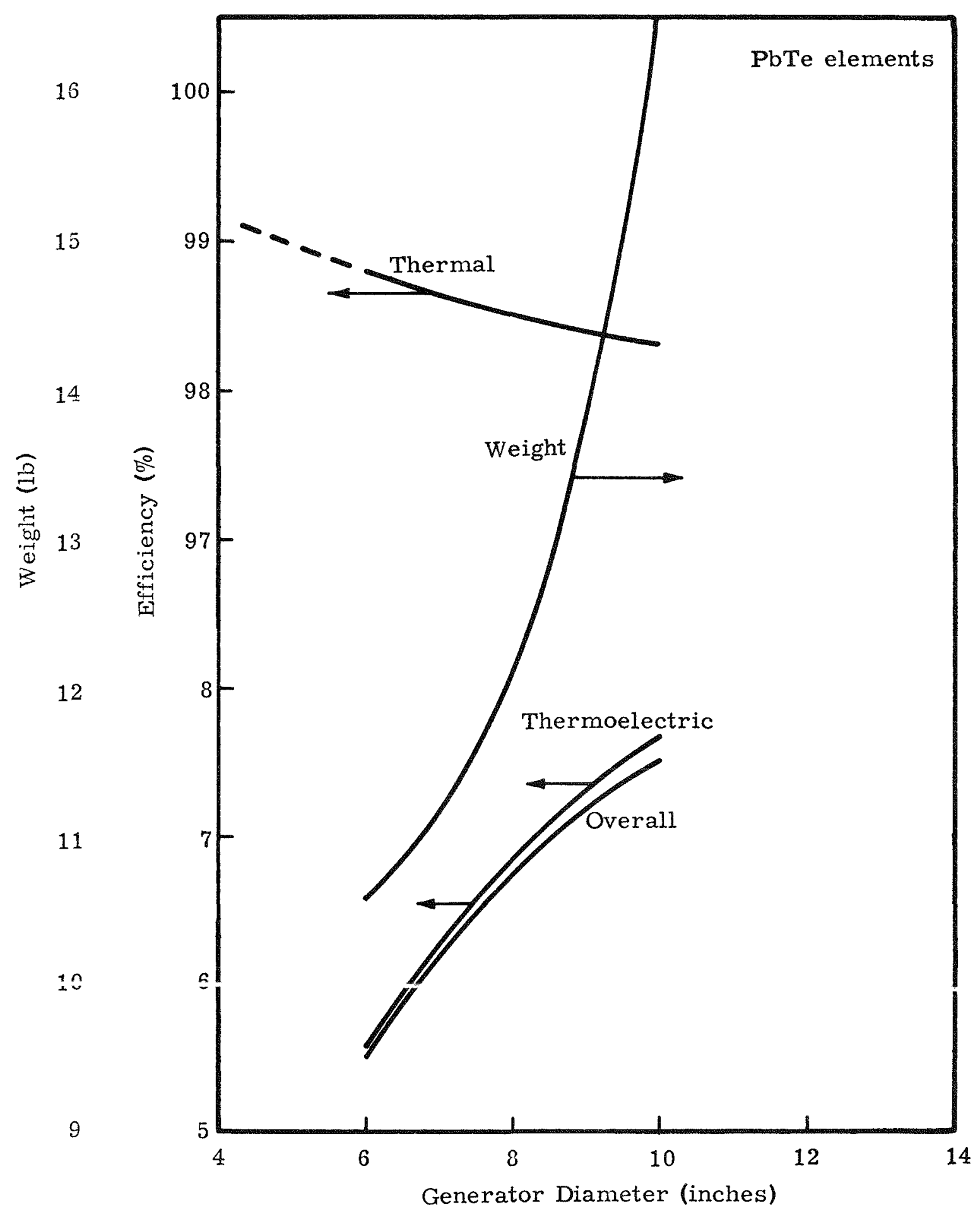

Fig. 85. Generator Diameter Versus Weight and Efficiency, Scaled-Up Model 
TABLE 12 (continued)

\begin{tabular}{|c|c|c|c|}
\hline & \multicolumn{3}{|c|}{ Unit Diameter } \\
\hline & 6 Inches & 8 Inches & 10 Inches \\
\hline $\begin{array}{l}\text { Thermoelectrics } \\
\text { Insulation }\end{array}$ & $\begin{array}{l}3.73 \\
1.77 \\
\end{array}$ & $\begin{array}{l}2.36 \\
3.94 \\
\end{array}$ & $\begin{array}{l}1.92 \\
7.39 \\
\end{array}$ \\
\hline Subtotal & 8.48 & 9.67 & 13.18 \\
\hline Structure $(25 \%)$ & 2.12 & 2.42 & 3.30 \\
\hline Total & 10.60 & 12.09 & 16.48 \\
\hline
\end{tabular}

Thermoelectrics

Output voltage

Number of pairs

Length (inches)

Area (sq in.)

$\begin{array}{lll}3 & 3 & 3 \\ 33 & 28 & 26 \\ 1 & 1 & 1\end{array}$
$P$ element
0.206
0.159
0.135
$\mathrm{N}$ element
0.168
0.131
0.113

4. Cylindrical-Type Unit with Lead Telluride Elements

Analysis. This design was based on maximum thermoelectric efficiency for a radiator area which was fixed essentially by the number of couples needed to produce the 13 watts of electrical power. The other factors dictating the radiator area were the 3.5 -inch radiation shield located between the fuel source and the counter, and the thermal loss through the insulation. The following assumptions were made in this design:

(1) The temperature drop through the hot and cold junction shoes is small compared to the $t \in$ mperature drop through the thermoelectric elements.

(2) No heat is lost except that which passes through the Min $\mathrm{K}$ insulation.

(3) The fuel elernent and radiator area have a uniform temperature.

This design has the following parameters:

(1) The hot junction temperature is $1000^{\circ} \mathrm{F}$. 
(2) The unit internal pressure is 0.5 atmosphere (air).

(3) The inner and outer shells are aluminum*.

(4) The heat source is shielded from the counter by 3.5 inches of gold. The shielding length is limited by the geometry of the generator.

Procedure. Using the fuel size determined for the three previous units, and incorporating the shielding requirements, the fuel element was sized. Next, a radiator area was chosen according to the number and length of thermal elements and a heat loss equation was derived (see Appendix E\%.

To begin this generator design, the method previously discussed in the procedure section for the radiative spherical-type unit was followed.

Summary of results. Results of the cylindrical-type unit investigated are shown graphically in Fig. 86, and are listed in Table 13 . It can be seen that, for the size units examined, the design requirement of 15 pounds maximum weight can be met and the overall efficiencies are better than $6 \%$.

TABLE 13

Cylindrical-Type Generator Specifications

Results

Unit Diameter (in.)

Efficiency (\%)

Thermoelectric

Thermal

Overall

Temperature $\left({ }^{\circ} \mathrm{F}\right)$

Hot junction

Cold junction

Heat loss (watts)
All Units 8 Inches in Height

$\begin{array}{lll}5.5 & 6 & 6.5\end{array}$

.5
6.72

91.3

6.1

6.83

91.4

6.2

6.97

92.6

6.4

Weight (pounds)

Heat source and container

Outer shell

2.41

2.41

2.41

Inner shell

0.68

0.83

0.93

0.34

0.32

0.30

* The inner shell will have to be made of a high temperature material. Since this has not been chosen, aluminum was assumed for weight calculation purposes. 
TABLE 13 (continued)

$\underline{\text { Results }}$

Thermoelectrics

Insulation

Structures $(25 \%)$

Total
All. Units 8 Inches in Height

\begin{tabular}{lll}
1.43 & 1.34 & 2.32 \\
1.53 & 2.01 & 2.51 \\
1.60 & 1.73 & 2.12 \\
\hline 7.99 & $\frac{1.64}{8.59}$ & $\frac{10.59}{}$
\end{tabular}

Thermoelectrics

$\begin{array}{llll}\text { Number of pairs } & 29 & 28 & 28 \\ \begin{array}{l}\text { Length (in.) } \\ \text { Area }\end{array} & 0.75 & 0.75 & 1.00 \\ \quad \text { P element (sq in.) } & 0.1218 & 0.1194 & 0.1485 \\ \quad \text { N element (sq in.) } & 0.1019 & 0.0984 & 0.125\end{array}$

Calculated thermal conductivity

$\begin{gathered}\text { Temperature } \\ \left({ }^{\circ} \mathrm{C}\right)\end{gathered}$
-100
0
100
200

$\mathrm{k}$

(watts $/ \mathrm{cm}-{ }^{\circ} \mathrm{C}$ )
0.04319
0.05425
0.06202
0.06804

5. Cylindrical Radiative-Type Unit with Cobalt Silicide Elements

Analysis. This design is basically the same as the cylindrical unit previously discussed except for the use of cobalt silicide instead of lead telluride for the thermoelements.

In analyzing the thermoelectric efficiency of the cobalt silicide elements for this design, the curves of thermoelectric power and resistivity from Transitron's report, "Thermal and Electrical Properties of Some Silicides," and the thermal conductivity which was calculated using the Wiedemann-Franz ${ }^{2}$ ratio were used. Employing these curves, the maximum thermoelectric efficiencies for a hot junction temperature of $1400^{\circ} \mathrm{F}$ were assumed and several cold junction temperatures were determined. 


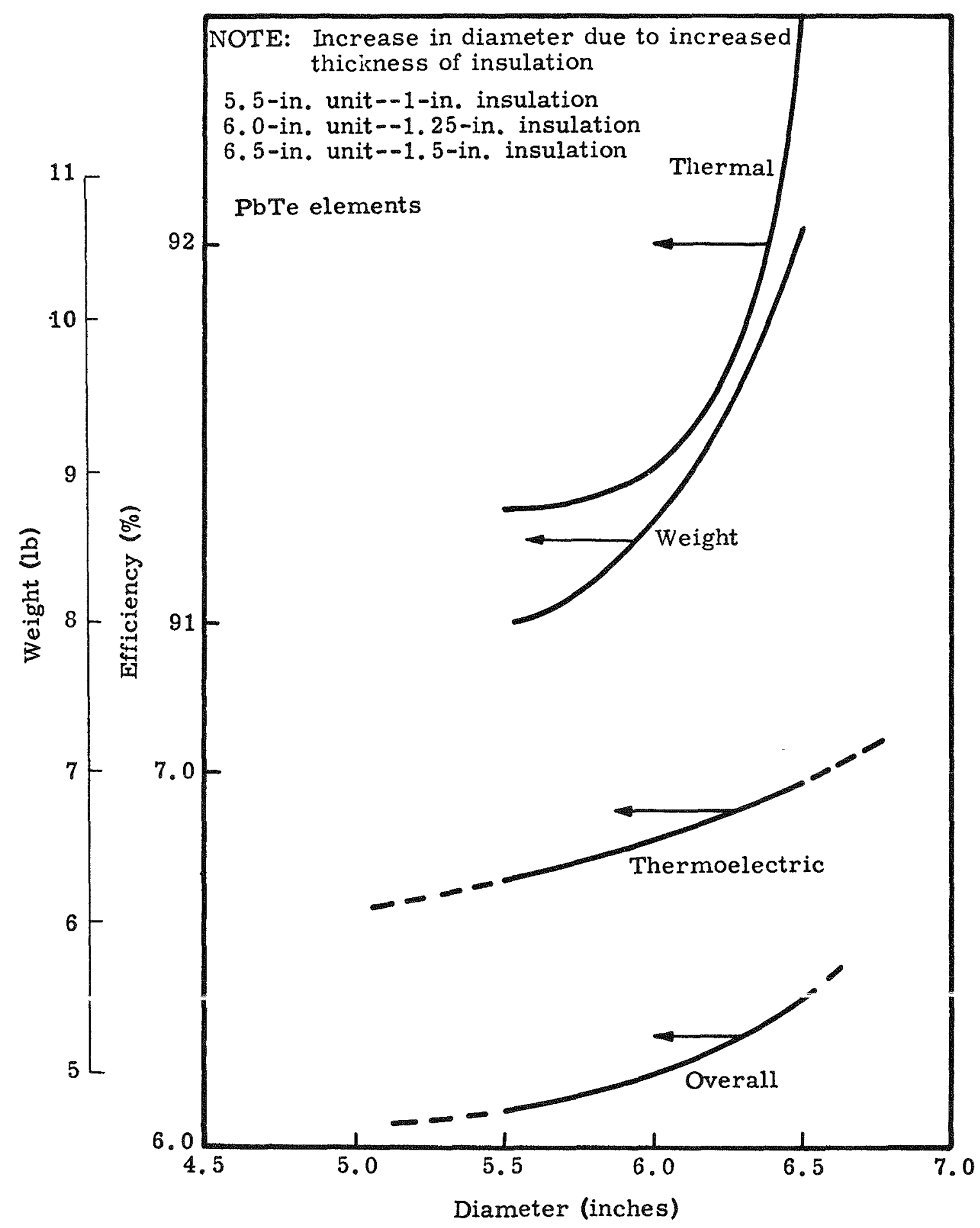

Fig. 86. Generator Diameter Versus Weight and Efficiency, Cylindrical Unit 


\section{Results}

Unit size--6-in. diameter, 7.5-in. height

$\begin{array}{lccc}\text { Hot junction temperature }\left({ }^{\circ} \mathrm{F}\right) & 1400 & 1400 & 1400 \\ \text { Cold junction temperature }\left({ }^{\circ} \mathrm{F}\right) & 343 & 300 & 200 \\ \text { Thermoelectric efficiency }(\%) & 6.3 & 7.22 & 7.29 \\ \text { Thermal conductivity- }-20^{\circ} \mathrm{C} \quad \begin{array}{c}0.04 \text { watts } / \mathrm{cm}-{ }^{\circ} \mathrm{C} \\ \text { (Transitron's value) }\end{array} & \begin{array}{c}0.056 \text { watts } / \mathrm{cm}-{ }^{\circ} \mathrm{C} \\ \text { (calculated) }\end{array}\end{array}$

Since Transitron's thermal conductivity data for cobalt silicide were quite scattered, they are not listed in this report. The value at room temperature was approximately 0.04 watts $/ \mathrm{cm}-{ }^{\circ} \mathrm{C}$, but the calculated value was 0.056 watts $/ \mathrm{cm}^{\circ}{ }^{\circ} \mathrm{C}$. Because of the poor correlation, no attempt was made to renormaliz e the calculated thermal conductivity for the cylindrical design, and a detailed analysis of this design was not attempted.

\section{Heat Control Analysis}

An attempt was made to adapt a gas leak-type heat dump mechanism to the cylindrical-type generator. The generator which was analyzed is shown in the sketch below.

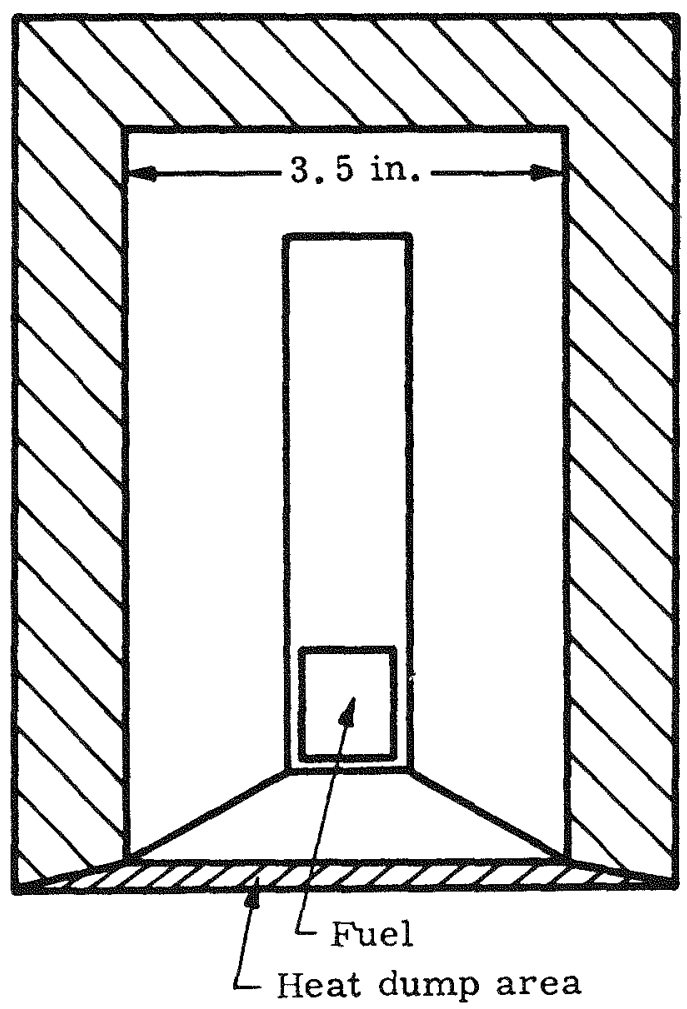


Blank page 
The calculated maximum heat that can be dumped for a temperature difference of $600^{\circ} \mathrm{F}$ and a wall thickness (including insulation filled with helium gas) of $3 / 16$ inch is approximately 37 watts. Therefore, it is possible to use this technique on a generator which requires less initial isotope fuel, but for the present design some other type of control will be required.

The combined Min-K and helium gas thermal conductivity was approximated, and this value was used to calculate the heat that could be dumped. Several temperature differences were assumed and the results tabulated. These results are shown graphically in Fig. 88 .

The temperature gradient in the fuel element can be approximately determined by assuming the temperature varies only in the axial direction as shown in the sketch below. Then, the differential equation describing the cylindrical fuel element can be shown to be:

$$
\frac{d^{2} T(x)}{d x^{2}}=\frac{k P}{k A} T(x)-T_{\infty}
$$

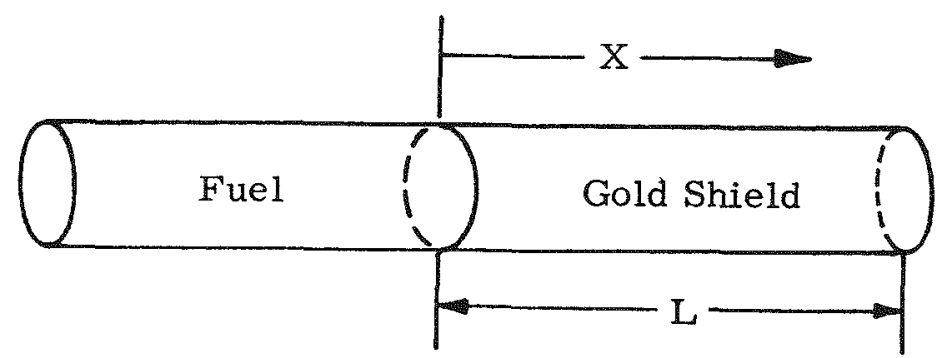

The solution to this equation is:

$$
T(x)=T \infty+C_{1} e^{n x}+C_{2} e^{-n x}
$$




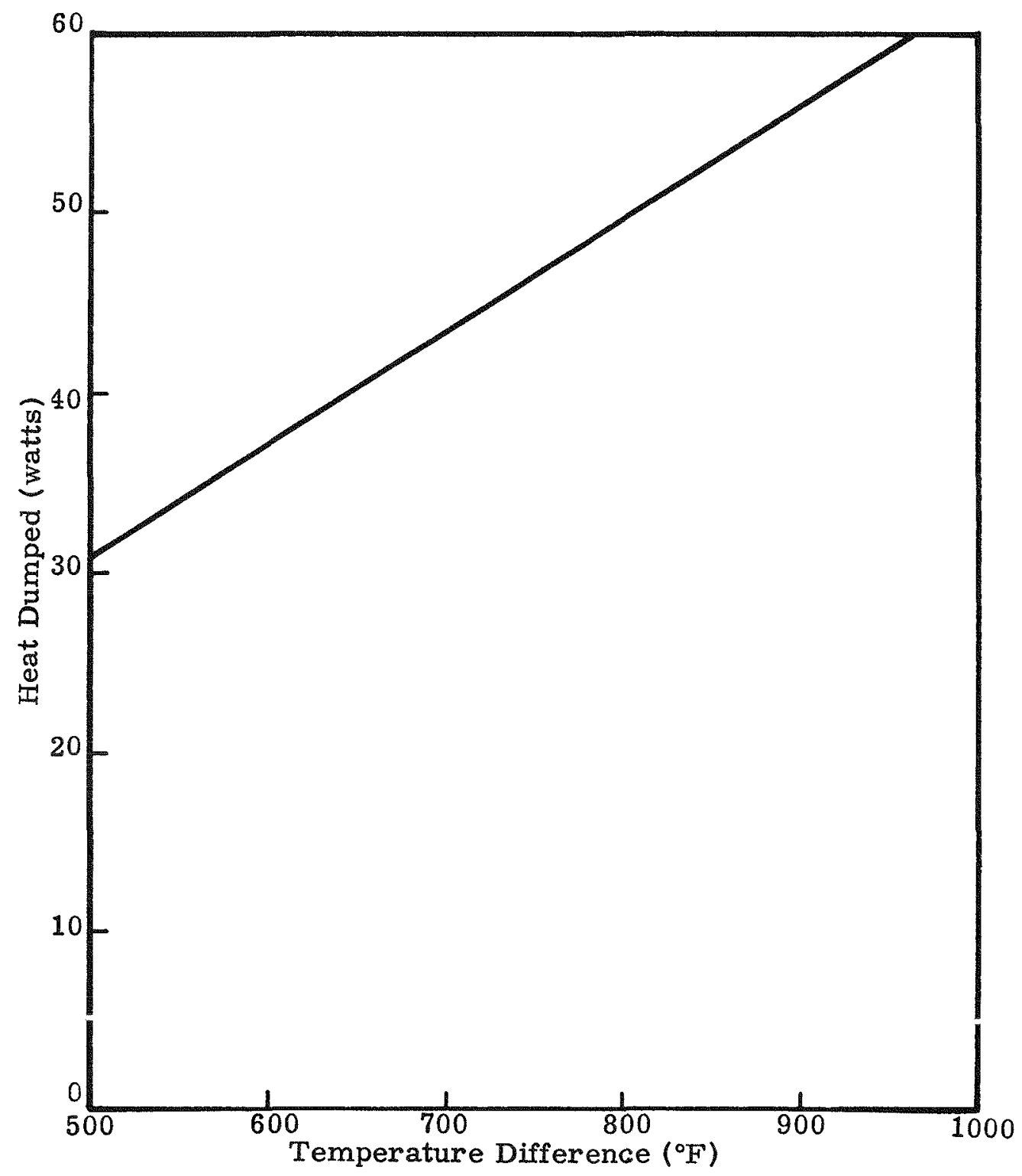

Fig. 88. Heat Dumped Versus Temperature Difference for Cylindrical Type Unit-Gas Leak-Type Dump 
Heat Source Temperature Distribution Data
$A \quad=\operatorname{cross}$ section (sq in.)
$\mathrm{L}_{\mathrm{o}} \quad$ = fuel length (in.)
$\frac{\text { Cylinder }}{0.196}$
L $\quad=$ Gold shield length (in.)
2.38
Fuel centerline temperature $\left({ }^{\circ} \mathrm{F}\right)$
1.5
Temperature at surface of block, midplane $\left({ }^{\circ} \mathrm{F}\right)$
1600
1375

NOTE: These results were obtained for an impacted fuel form consisting of one part Curium-242 and eight parts (by atomic ratio) of platinum. The heat source will be redesigned for an alloy of Cm-242 and nickel, or for use of pure curium.

where

$$
\begin{aligned}
T_{\infty} & =\text { surroundings temperature } \\
T(x) & =\text { temperature as a function of } x \\
C & =\text { constant of integration } \\
n & =\frac{h P}{k A} \\
h & =\text { combined coefficient of radiation and convection } \\
P & =\text { perimeter } \\
k & =\text { thermal conductivity } \\
A & =\text { cross-sectional area }
\end{aligned}
$$

The constants of integration can be evaluated by choosing the temperature at $x=0$ to be $T_{0}$ and by assuming that the film coefficient concept applies to the removal of heat at $x=L$.

$$
\begin{aligned}
& C_{1}=\frac{T_{0}-T_{\infty}}{1-a e^{-2 n L}} \\
& C_{2}=\frac{T_{0}-T_{\infty}}{1+a e^{-2 n}} \\
& a=\frac{n k-k}{n k+h}
\end{aligned}
$$

The requirement for the gold shield is discussed in Section C of this chapter.

The numerical data and results for the heat source of the cylindricaltype generator configuration are listed in Table 14. 


\section{B. GENERATOR AND COMPONENT DESIGN STUDIES*}

Design studies during October and November 1959 were directed to transforming the results of analysis of the various generator configurations into conceptual designs which show the general arrangement of components and permit reasonably accurate weight estimates. The designs are shown in Figs. 83 and 89 through 93. The multiple SNAP-III configuration is indicated by the plan and elevation drawings of the SNAP-III-B generator; the five units might be located, e.g., at the corners and centroid of a regular tetrahedron.

A concept was evolved for a generator that approximates a sphere in shape but in actuality is a polyhedron. The flat surfaces would allow the thermoelements to be seated without use of curved hot shoes or some other arrangement involving curved surfaces. The 14 -sided polyhedron (tetradecahedron) is sufficiently like a sphere that the heat transfer analysis of the spherical generator is applicable.

Two additional design concepts were examined briefly:

(1) A generator design of two concentric spheres, the inner one of titanium and the outer of aluminum. The inner sphere would be designed so that a gas pressure inside would expand the sphere and cause the thermoelements to make good physical contact between the hot and cold junctions. This might eliminate the requirement for springs on the cold ends of the elements.

(2) Utilizing the concentric shells as mentioned above, a bellows-type arrangement would be attached to the cold ends of the elements. The sealed bellows would contain a thermally conductive liquid or gas. The bellows would expand due to a temperature increase during operation and press against the radiator surface, forming a conductive heat path and also allowing for thermal expansion of the element.

Neither of these concepts appears to offer sufficient rigidity to withstand the dynamic environment conditions.

Detailed design of the cylindrical-type generator, chosen as the optimum configuration, was carried on during December 1959. Since this work was in progress at the end of the quarter, its results will be described in the next report. 

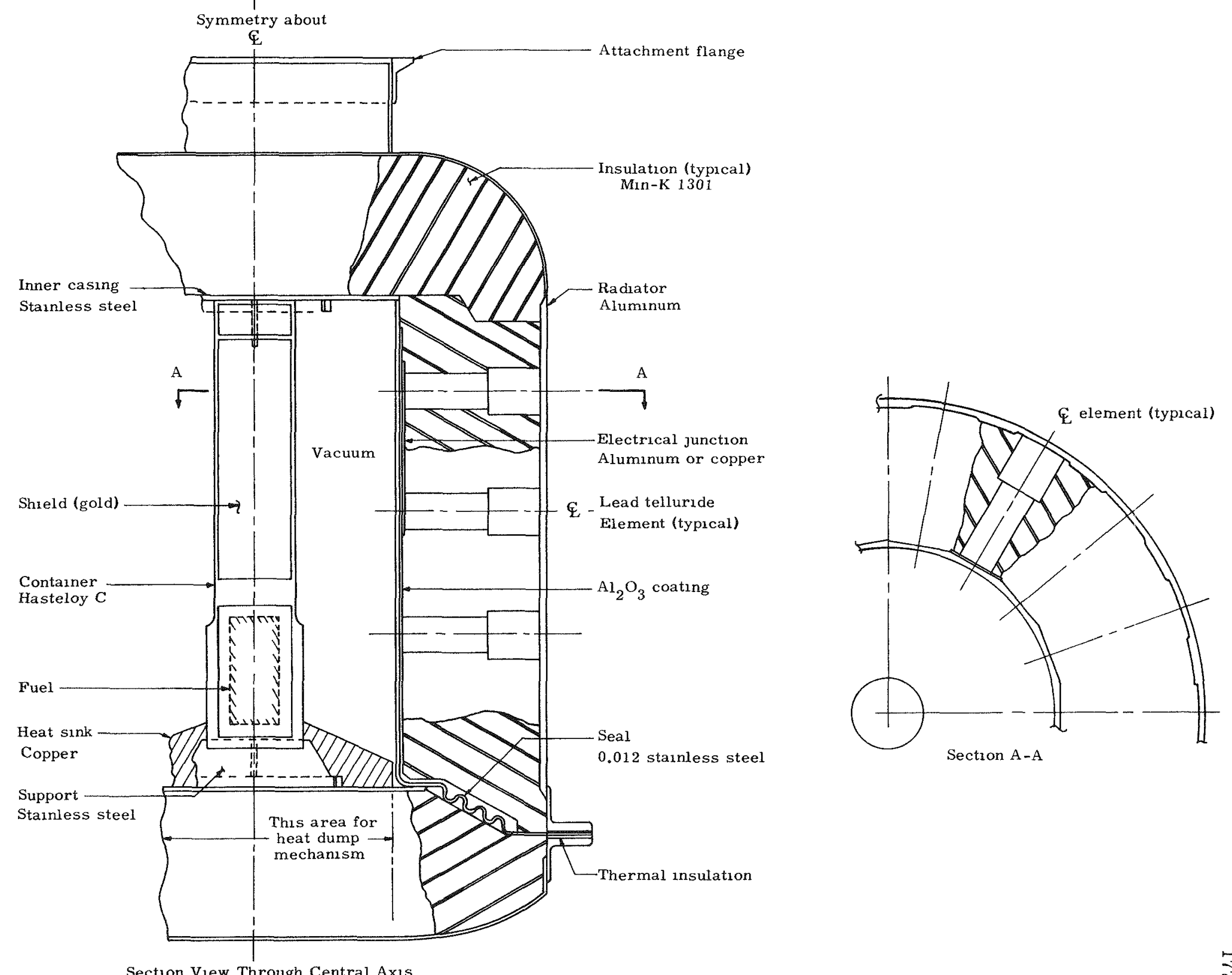

Section View Through Central Axıs

Fig. 89. Conceptual Design Cylindrical 13-Watt Generator 


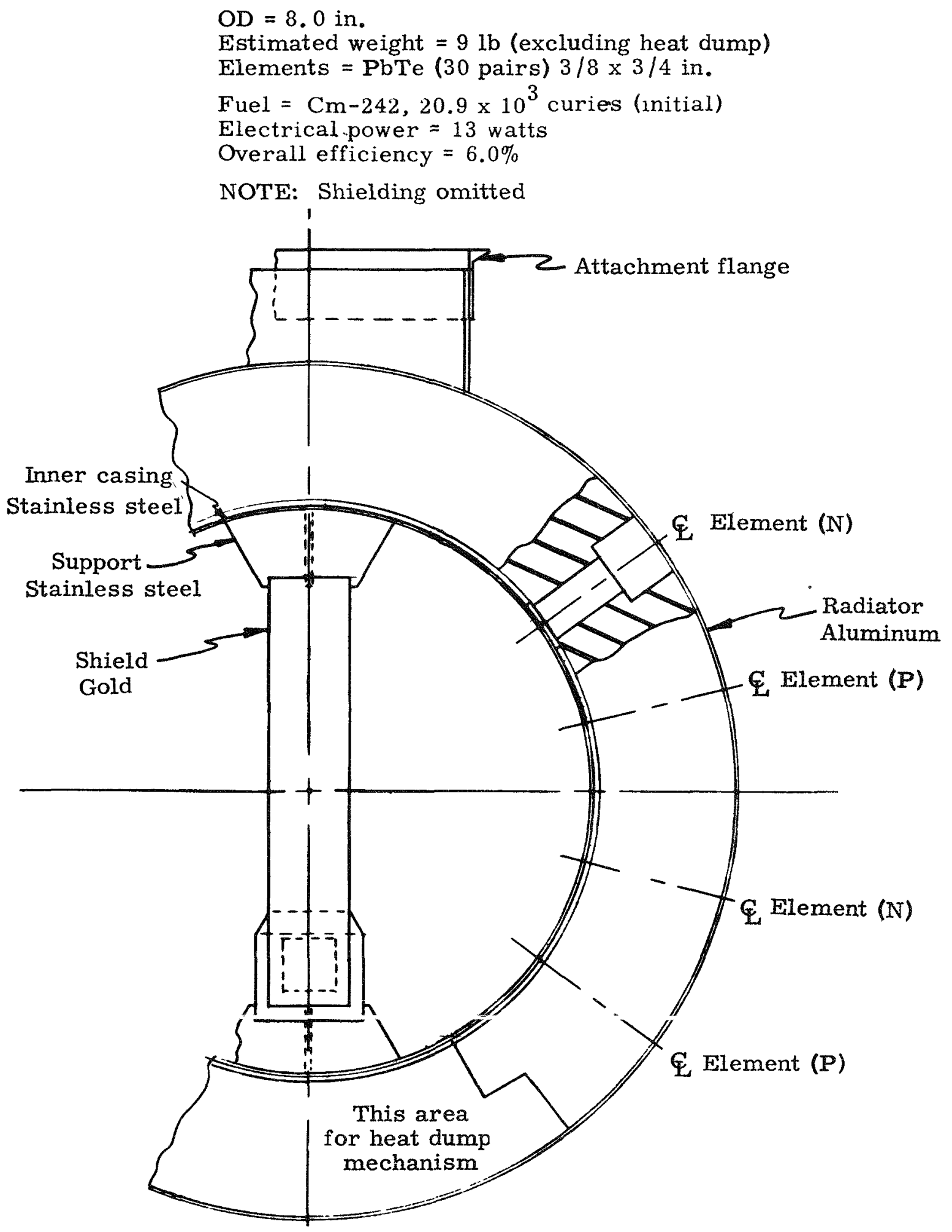

Symmetrical about

⿷

Fig. 90. Spherical 13-Watt Generator 
$\mathrm{OD}=6-3 / 4 \mathrm{in}$.

LOA $=9$ in.

Estimated weight $=9 \mathrm{lb}$ (excluding heat dump)

Elements $=\mathrm{PbTe}$ (30 pairs)

Fuel, Cm-242,20.9 × $10^{3}$ curies (initial) 1)

Electrical power, 13 watts

Overall efficiency $=6.0 \%$

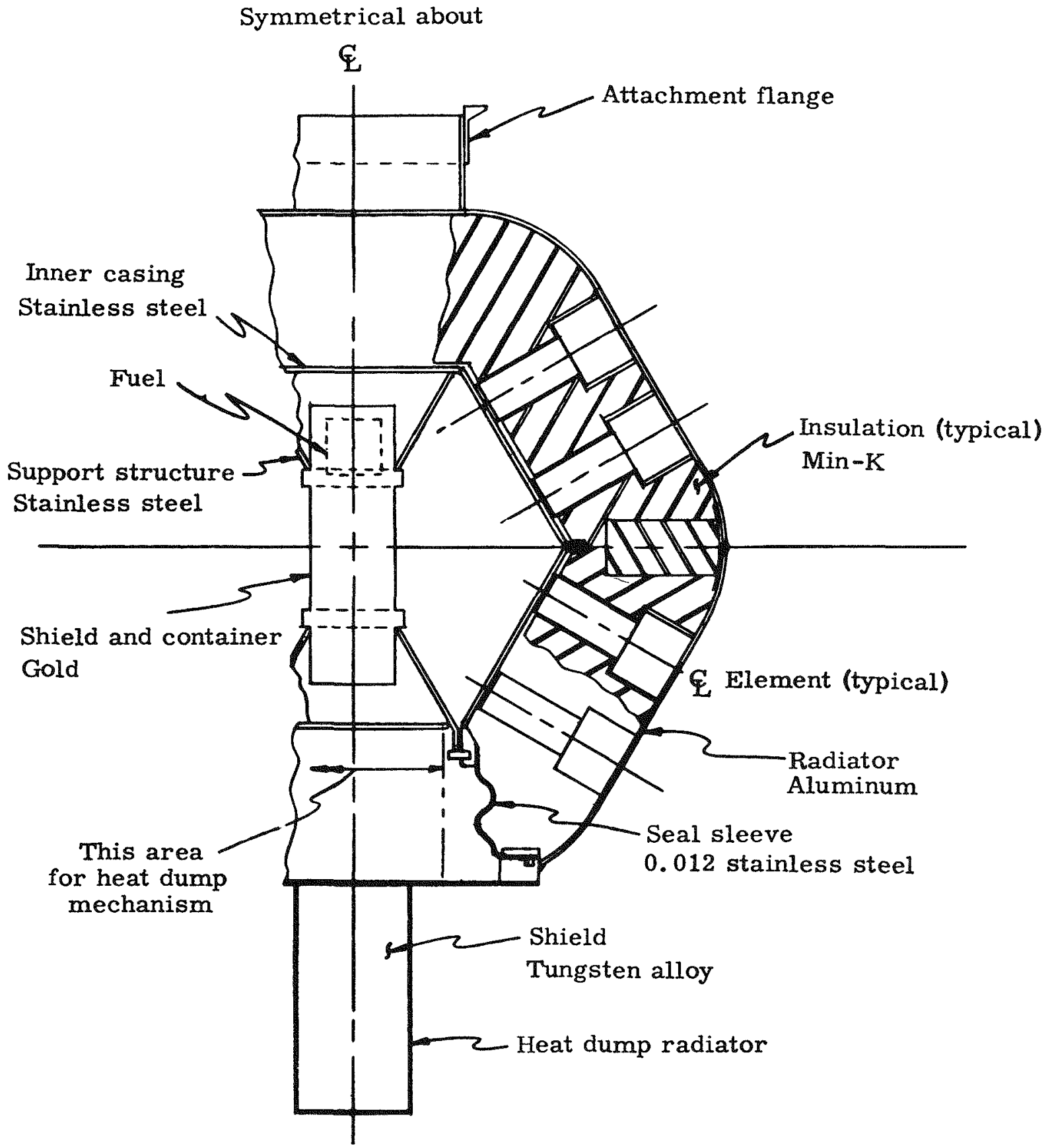

Section through central axis

Scale 1-1

Fig. 91. Modified Sphere 13-Watt Generator 


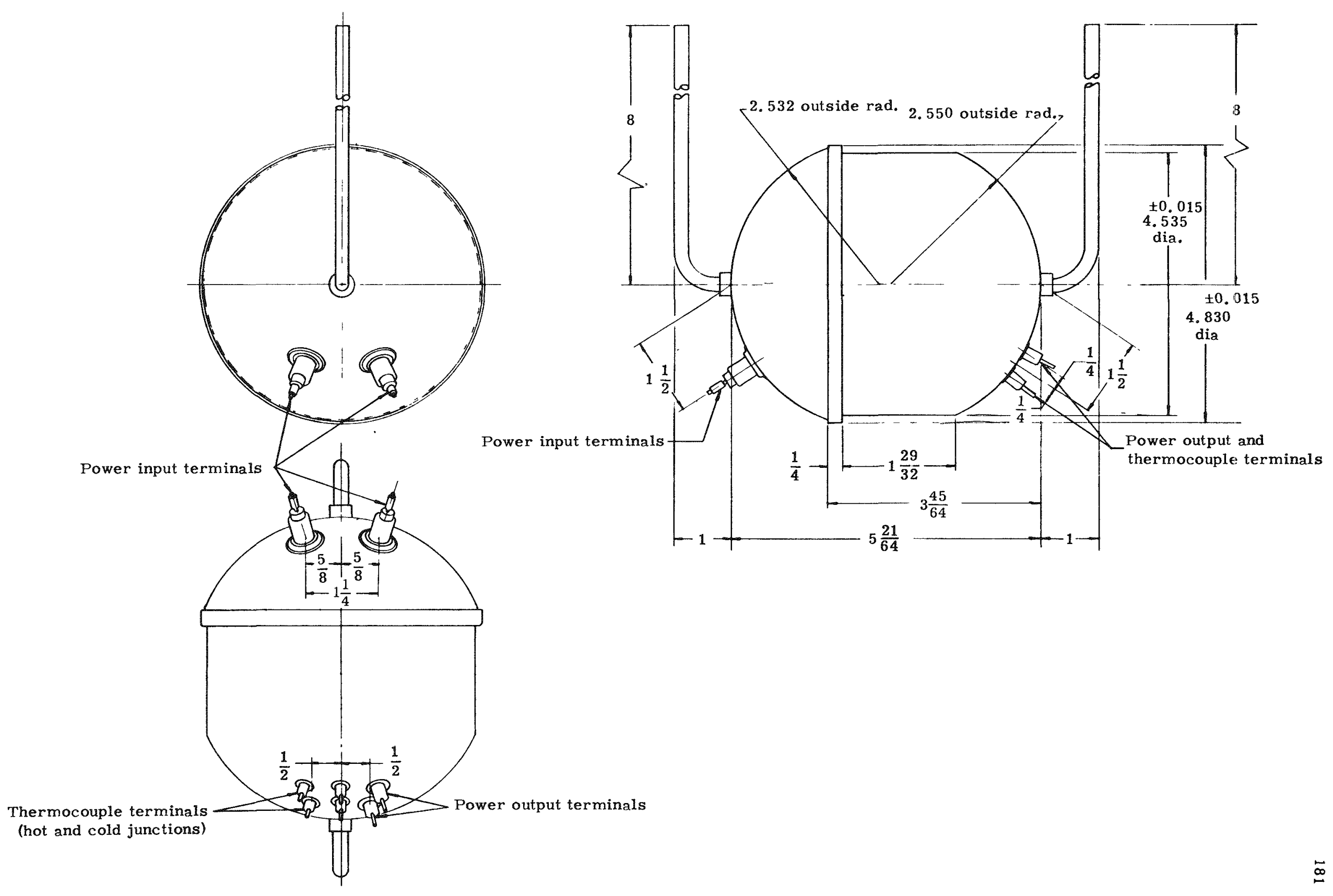

Fig. 92. SNAP III Thermoelectric Generator 
An investigation previously initiated in the Task 2 (SNAP-I-A) program, that of flame spraying metals for hot shoes over a surface previously coated with $\mathrm{Al}_{2} \mathrm{O}_{3}$ as a dielectric, is being continued. This technique may eliminate difficulties in hot shoe contact with the hot junction, it also insulates electrically while providing an electrical conduction path between elements in a pair.

Work remains to be done in testing the stainless steel sample pieces in the laboratory. Tests to be conducted include resistivity of the sprayed material, heat conductance and electrical conductance through the sample assembly, and a high temperature test for spalling of the dielectric material. Sample pieces have been made with stainless steel as the base metal and $\mathrm{Al}_{2} \mathrm{O}_{3}$ as an insulator. These samples have been coated with aluminum and copper. Attempts to spray iron onto the oxide coating have not been successful. One more sample is to be made of molybdenurn.

The resistivity of the aluminum-and copper-coated samples has been measured. As would be expected for a spray coating, the resistivity values are higher than those given for standard rolled materials due to the differences in density. Measurements will be taken after the samples have been heated in the furnace and these values may change slightly. Since the measurements were incomplete at the end of the quarter, quantitative results will not be presented here.

Work remains to be done in testing the stainless steel sample pieces in the laboratory. Tests to be conducted include resistivity of the sprayed material, heat conductance and electrical conductance through the sample assembly, and a high temperature test for spalling of the dielectric material.

A conceptual design was prepared for a ground handling cask for trans porting the 13-watt generator, complete with Curium-242 fuel container, from the Martin plant to the launch site. The design is shown in Fig. 93.

The generator compartment in the cask is completely surrounded by a 4 -inch layer of water for biological shielding. The design shielding thickness for borated water is 3.5 inches. Four inches of water, an equivalent thickness, was adopted so that the cask may be filled with tap water. The preliminary design makes use of a refrigeration coil (Freon 12) around the outside of the generator. At first, the concept was that refrigeration would be needed to convey heat from the generator side walls, but later heat transfer calculations indicated that this was not necessary. There is not enough heat transferred to the shielding to cause it to boil.

Pressure relief valves have been installed in the water compartments as a safety measure to prevent vapor pressure buildup in case of steaming. 


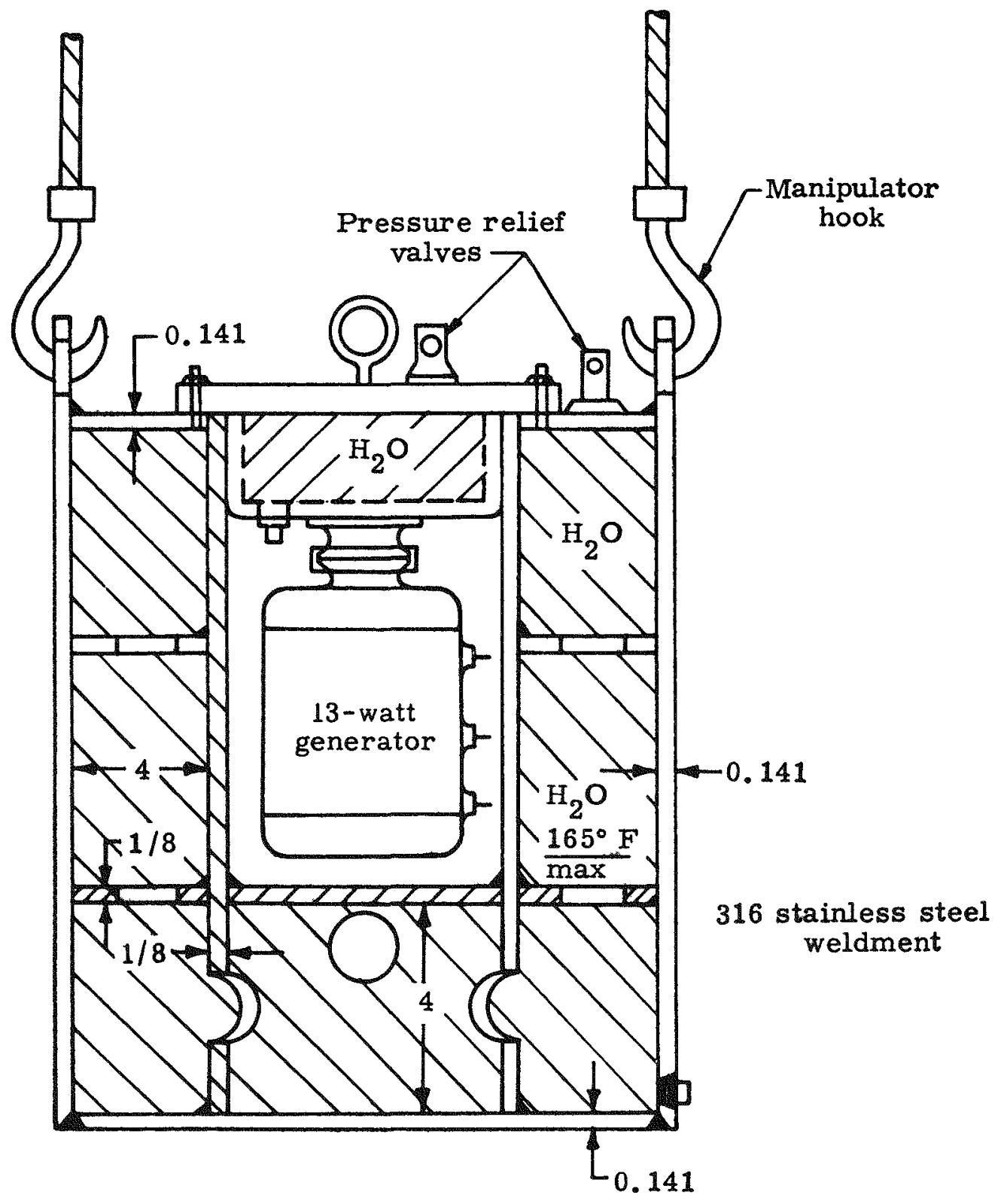

Gross wt: 270 1b

Fig. 93. 13-Watt Generator Ground Handling Cask 
Pipe plugs have been provided for the filling and draining of water. Two hook eyes for the manipulators are located in the main body of the cask and also in the shielded lid to facilitate installing and removing the generator from the ground handling cask.

The gross weight of the assembled cask including 16 gallons of water, but exclusive of generator, is approximately 255 pounds. The empty cask weighs 122 pounds.

The calculated overall efficiency of the spherical generator, $6.0 \%$, established the initial thermal power of 602 watts. Converting this into fuel quantity yielded a requirement of $1.96 \times 10^{4}$ curies, or 6 grams of $\mathrm{Cm}-242$.

The radiation sources considered were:

(1) Fast neutrons from spontaneous fissioning of $\mathrm{Cm}-242$ and neutrons from $(\alpha, n)$ reactions if the fuel compound contains light elements.

(2) Gamma rays from alpha disintegrations which lead to excited states of $\mathrm{Pu}-238$.

(3) Prompt gamma rays from spontaneous fission.

(4) Gamma rays from neutrons inelastically scattered by the lead telluride thermoelectrics.

The ground handling requirement does not present a major problem. The largest contribution to the total dose rate is from the fast neutrons, the next significant contribution to the total dose rate is from gammas associated with alpha decay, and the least significant contribution is produced by the prompt fission gammas. The gammas from inelastically scattered neutrons are insignificant in the calculation of ground handling dose rates. Table 14 shows the calculated dose rates (at three feet) and thicknesses of water. 
TABLE 15

Thicknesses of Water and Dose Rates

Frum fast neutrons

From decay gammas

From fissioning gammas

Total mrem/hr

\author{
Curium Metal in \\ $3-1 / 2$ in. of Bo- \\ rated Water \\ (mrem/hr)
}

\section{7}

3.2

$\frac{1.1}{60}$

Curium Carbide in 5-3/4 in. of Water (mrem/hr)

\subsection{5}

1.85

0.80

The use of borated water or an aqueous solution of a lithium salt will reduce the shielding thickness by several percent.

The gamma shielding requirements imposed by the operational specifications are severe, and calculations showed that the power unit weight limitation could be met only by increasing the distance of the detector from the heat source.

\section{NUCLEAR RADIATION SHIELDING*}

A calculation of the shielding requirements for fast neutrons was made using the removal cross-section concept. This calculation gave a dose rate at one meter of $60 \mathrm{mrem} / \mathrm{hr}$ when 2.5 inches of water were added to the 10-inch diameter generator.

The following analysis of the shielding requirements for a 13-watt generator fueled with Curium-242 was made for a spherical generator 10 inches in diameter. With the exception of the shadow shield weights, the information is applicable to generators of other shapes.

The lifetime of the unit is 240 days, of which 60 days have been allowed for fabricating, shipping, storage, etc. Allowable radiation levels have been specified as being $60 \mathrm{mrem} / \mathrm{hr}$ at one meter during ground handling. An additional specification applicable during the useful life of the unit requires shielding for a radiation detector located $10 \mathrm{~cm}$ from the surface of the generator. Shielding is required to reduce the photon flux to 7 photons $/ \mathrm{cm} / \mathrm{sec}$ with energies greater than $100 \mathrm{kev}$. The total weight must not exceed 15 pounds. 
The photons which reach the counter come from a number of sources. The most numerous photons are those which originate in the fuel (i.e., the decay gammas and the prompt fission gammas). The next most numerous photons are those from neutrons scattered inelastically in the thermoelectric elements. Another source of photons, those scattered from the structure, is small and cannot be evaluated without some knowledge of the structure surrounding the generator.

The gammas originating in the source could be reduced by a shadow shield built within the generator. In this case, an additional shield would have to be provided to reduce the gammas from the thermoelectric elements. This shield could be either a shield around the source to reduce the number of neutrons available for production of gammas or a shadow shield in front of the detector. The first method was not considered because calculations made for the ground handling requirement indicate that the shielding weight would be excessive. The shield for the latter method would be in the shape of a frustum of a cone defined by the outer diameter of the generator and the end of the detector farthest from the generator. However, in order to meet the weight requirements, the location of the detector must be changed. The configuration and total weight of the divided shield at various distances from the source are shown in Fig. 95.

Gold was selected for the shield inside the generator because of its high gamma absorption coefficient and high thermal conductivity; the latter promotes uniformity of hot junction temperatures. The melting temperature of gold, $1062^{\circ} \mathrm{C}$, permits it to remain solid under normal conditions but to melt during re-entry. The cost of the gold is less than $\$ 1000$ 。

Another method of reducing the photon flux to the detector would be to use one shield for both the source and inelastic gamma photons. Figure 96 shows the configuration and gives the shield weights for various sourceto-detector distances. The weights of the divided shields are less in all cases. Note that the weights were determined for a 10-inch diameter generator and could be reduced by reducing the diameter of the generator.

\section{RADIATION SOURCES}

\section{Fast Neutron Intensity}

One gram of $\mathrm{Cm}-242$ will produce $1.97 \times 10^{7}$ neutrons per second per gram by spontaneous fissioning (Ref. 3). Consequently, the generator under consideration will emit $6 \times 1.97 \times 10^{7}=1.18 \times 10^{8}$ fission neutrons per second. 


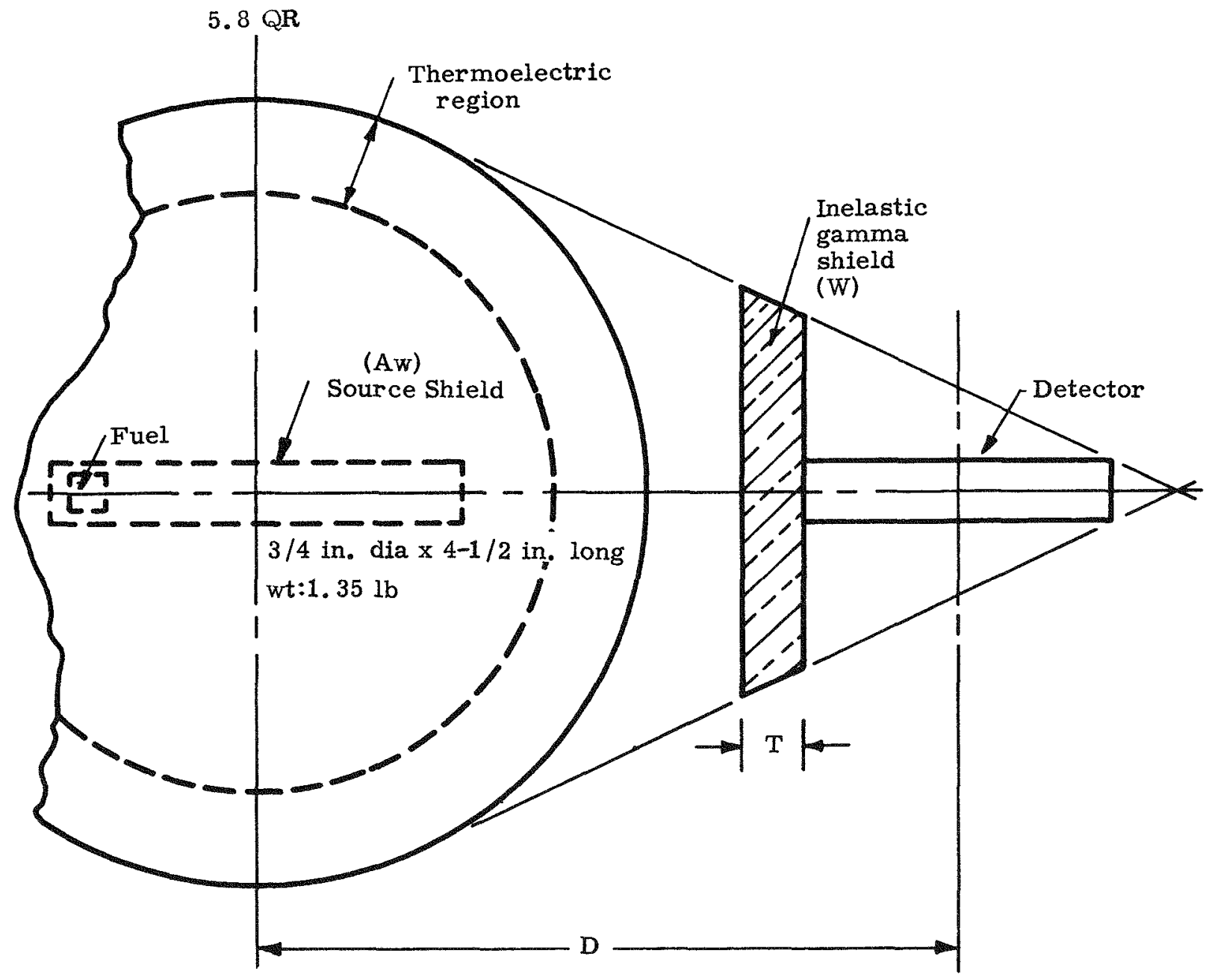

Weight of Divided Shield for Various Distances

$\begin{array}{llcl}\begin{array}{l}\text { Distance } \\ \text { D (inches) }\end{array} & \begin{array}{l}\text { Thickness } \\ \text { T (inches) }\end{array} & \begin{array}{c}\text { Total Weight } \\ \text { (pounds) }\end{array} \\ 8.94 & 25 / 8 & 45 & 10 \mathrm{~cm} \text { from surface } \\ 20 & 13 / 4 & 15 & \\ 30 & 13 / 8 & 8.9 & \\ 40 & 11 / 8 & 6.1 & \\ 50 & 1 & 5.2\end{array}$

Fig. 95. Geometry for Divided Shield 


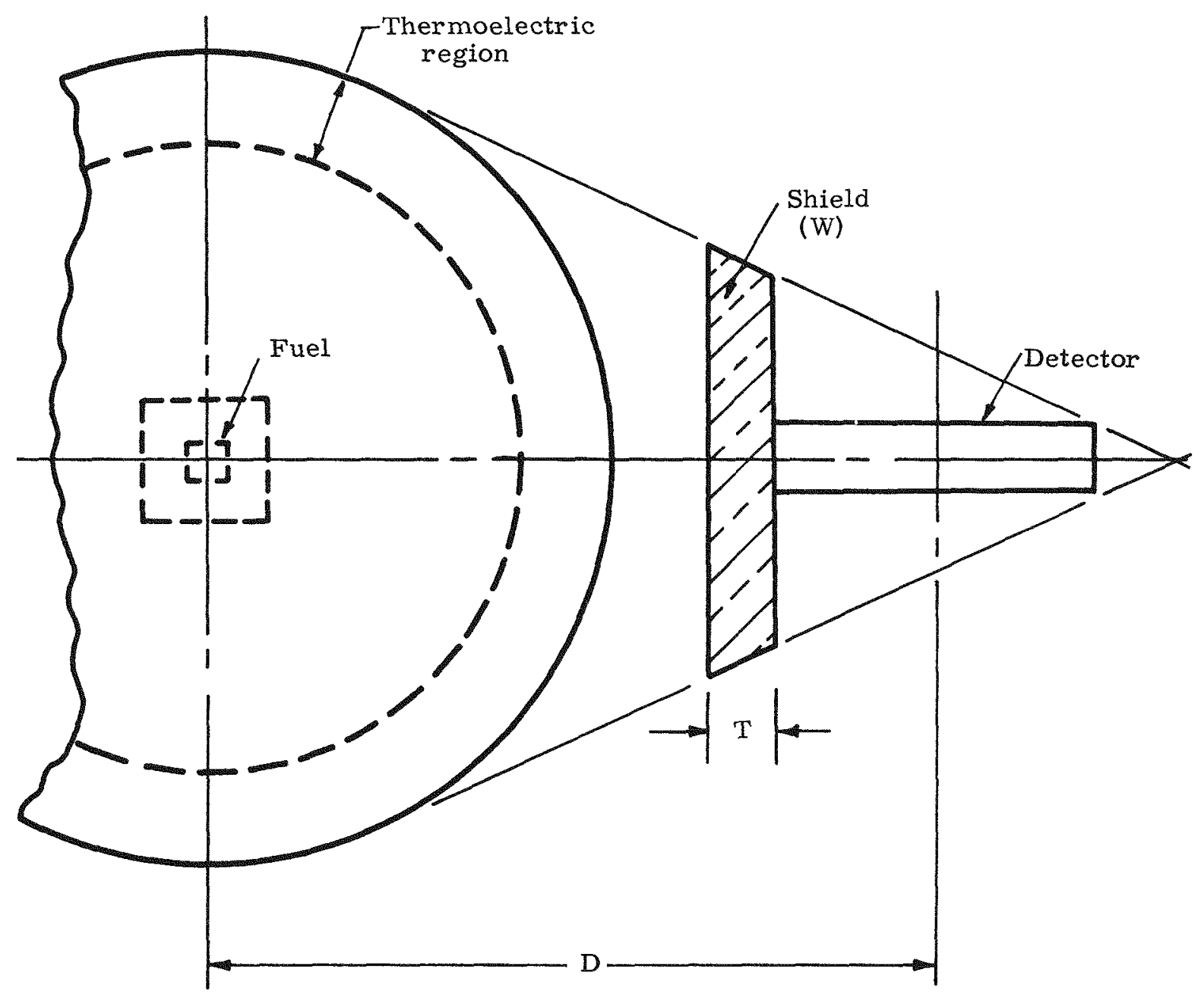

Weight of Unit Shield For Different Distances

$\begin{array}{lcc}\begin{array}{l}\text { Distance } \\ \text { D (inches) }\end{array} & \begin{array}{c}\text { Thickness } \\ \text { T (inches) }\end{array} & \begin{array}{c}\text { Weight } \\ \text { (bounds) }\end{array} \\ 8.94 & & -- \\ 20 & 2.75 & 23.0 \\ 30 & 2.50 & 14.7 \\ 40 & 2.75 & 10.3 \\ 50 & 2.00 & 7.6\end{array}$

* Shield is too thick to fit between detector and generator.

Fig. 96. Geometry For Unit Shield 
If the fuel is in the form of a compound (or mixture) containing light elements, additional neutrons will be produced from alpha-neutron reactions. For curium carbide, about 50\% of the neutrons emitted will be from alpha-neutron reaction with carbon (Ref. 4), and the total number emitted will be double the number from spontaneous fission. Because no information concerning the neutron spectrum from the alpha-neutron reaction was found, it was assumed that the distribution is the same as that for spontaneous fission neutrons, i.e., all neutrons are considered to be fission neutrons. Actually, the alpha-neutron reaction will harden the spectrum as more high energy neutrons will be present.

However, this assumption will not affect the dose rate too much, since the flux-to-dose rate conversion factor is almost constant for neutrons of energies between 1 and $10 \mathrm{Mev}$.

Evidence presented in Refs. 5 and 6 indicates that the fission neutron spectrum for all transuranium elements is nearly the same as that for U235. The integrated U-235 fission spectrum presented in Ref. 7 was used, and flux-to-dose rate conversion factors were obtained from Ref. 8.

Energies and abundances of gamma rays associated with alpha decay were obtained from Ref. 9 .

The assumption was made that prompt gammas from spontaneous fissioning of transuranic elements are nearly the same as those for thermal neutron fissioning of $U-235 *$. The abundance and energy distribution given in Ref. 9 were used to calculate the effect of this source of radiation.

2. Gammas from Inelastically Scattered Neutrons

Information on the cross sections for production of gamma rays by inelastically scattered neutrons is very incomplete and does not warrant detailed calculations of flux distributions. However, the nonelastic cross section (total cross section minus elastic scattering cross section) may be considered to be an overestimate of the inelastic scattering cross section in estimating gamma ray production at high energies (Refs. 10 and 11).

To estimate the effect of inelastic scattering gamma rays, the assumption was made that all nonelastic captures produce gamma rays at the energy of the minimum absorption coefficient of the shield material. The nonelastic cross sections for lead were used to estimate gamma ray production in the lead telluride thermoelectric elements.

The photon flux at $10 \mathrm{~cm}$ from the outer surface of the generator was estimated by assuming the thermoelectric elements to be located in a shell at the outer radius of the generator.

\footnotetext{
*This is not quite correct, based on UCRL measurements of the prompt neutron spectrum from the spontaneous fission of $\mathrm{C} f-254$.
} 


\section{E. HAZARDS EVALUATION*}

Much of the evaluation of hazards performed under Subtask 5.7 for the 100-watt thermoelectric generator is applicable to the present program. Only considerations and results peculiar to the 13 -watt power units are reported here.

Calculations of helium pressures to be expected were made on the following assumptions: the volume of the void provided is $2 \mathrm{cc}$ and its temperature is $866^{\circ} \mathrm{K}\left(1100^{\circ} \mathrm{F}\right)$; and the mass of Curium-242 is $5.73 \mathrm{gm}$, equivalent to 0.0237 moles. The pressure reaches 8000 psi in about 270 days, at which time it is increasing very slowly--about 5 psi per day. Figure 97 is a graph of the pressure against time for this unit. The calculations have been made for a constant temperature. If the temperature of the generator decreased, the pressure will be reduced proportionately. After 125 days, the pressure would reach 12,400 psi if the temperature were to remain at $866^{\circ} \mathrm{K}$. However, the temperature will have fallen almost to ambient at this time. For a temperature of $293^{\circ} \mathrm{K}\left(68^{\circ} \mathrm{F}\right)$ the pressure would be about 4200 psi.

Since the containment of the fuel on the moon after the useful life of the generator is ended is a matter of interest, it must be realized that the helium pressure will continue to build up indefinitely, but very slowly.

The fuel container will probably be a Hastelloy $\mathrm{C}$ right cylindrical vessel with an outside diameter of one inch and an inside diameter of $1 / 2$ inch. The vessel will be $4-1 / 4$ inches in length. The internal space not occupied by fuel or the required void may be filled with gold to serve as a shadow shield for instrumentation placed on that side of the generator.

The resistance to internal pressure of such a vessel may be calculated by the equation

$$
P=\frac{b^{2}-a^{2}}{b^{2}+a^{2}} v
$$

where

$$
\begin{aligned}
& P \quad=\text { pressure, psi } \\
& \text { a = inside radius of cylinder, inches } \\
& \mathrm{b}=\text { outside radius of cylinder, inches } \\
& v \quad=\text { ultimate tensile strength of the materials; } 5 \times 10^{4} \text { psi for } \\
& \text { Hastelloy } \mathrm{C} \text { at this temperature. }
\end{aligned}
$$

*G. Dix and C. Riggs 


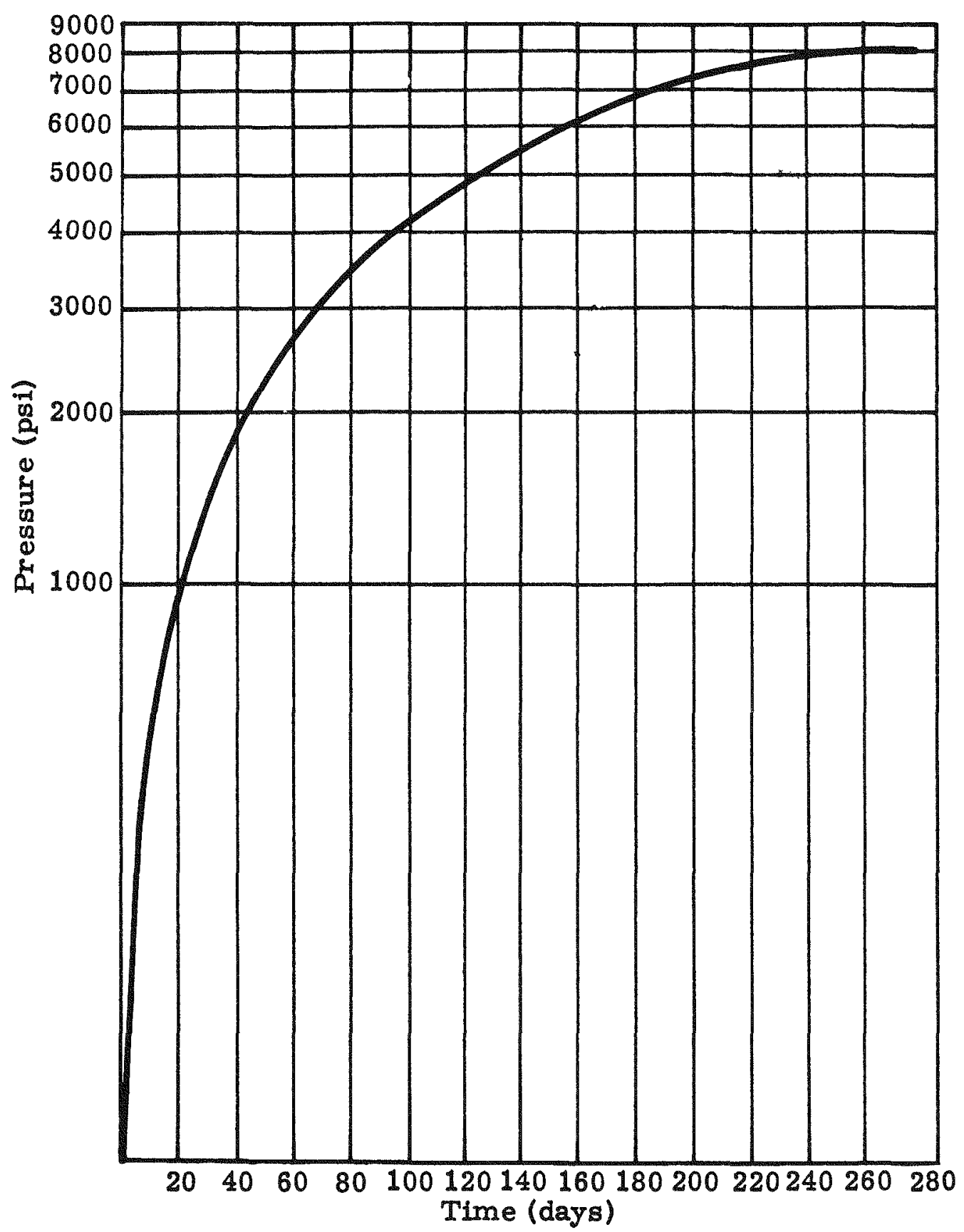

Fig. 97. Buildup of Helium Pressure With Time 
Applying this equation to the case considered, we find that it should withstand an internal pressure of 30,000 psi and have a large factor of safety for the helium pressures that have been found. 
IX. SUBTASK 6.1--GENERAL DEVELOPMENT AND MATERIALS REQUIREMENT

The purposes of this subtask are:

(1) To establish potential areas of study for the development of suitable fuel forms of Curium-242 and Plutonium-238..

(2) To determine the fuel compounds which will provide the desired physical and chemical characteristics.

(3) To select the compounds which will meet the requirements of the particular application.

\section{A. MATERIALS EVALUATION}

A literature search for the physical properties and methods of forming curium and plutonium was started during the latter part of this period. One of the objectives of this subtask is to consolidate into tables the available data on the physical, thermal and nuclear properties of the radioisotope fuels. Table 16 summarizes most of the available literature on Curium-242, Plutonium-238, Cerium-144, Polonium-210, Strontium-90, Promethium-147 and Cesium-137.

An investigation of matrix materials in conjunction with some of the fuels for thermoelectric systems was made to determine the naturally cooled surface temperatures of fuel slugs fabricated with matrix materials having optimum properties of density, melting point and thermal conductivity. Table 17 and Fig. 98 show the properties and surface temperatures, respectively, of gold, copper, nickel, platinum, molybdenum, iron and graphite.

In general, the thermal conductivities of the oxides of the radioisotopes are considerably lower than that of the pure metals. The carbides usually have higher thermal conductivities.

In Table 17 some of the more promising matrix materials for Curium-242 are listed. A fuel which combines the following properties is being sought for use in thermoelectric generators:
(1) Low density.
(2) Melting point of fuel matrix --2500 to $3000^{\circ} \mathrm{F}$.
(3) High thermal conductivity. 
TABLE 16

Physical Properties of Radioisotopes

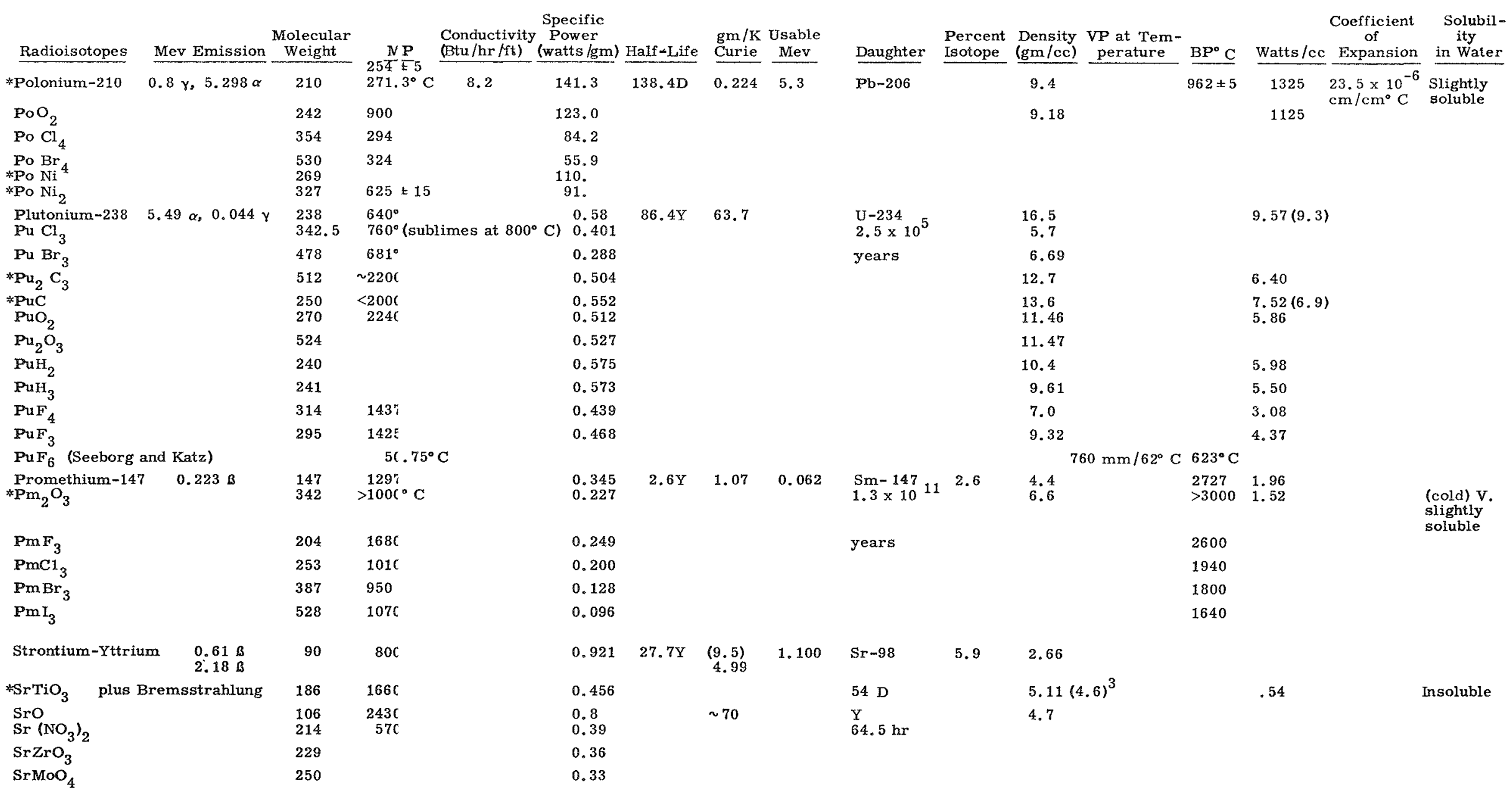


TABLE 16 (continued)

\begin{tabular}{|c|c|c|c|c|c|c|c|c|c|c|c|c|c|c|c|c|c|}
\hline Tradiousotopes & Mev Emission & $\begin{array}{l}\text { Molecular } \\
\text { Weight }\end{array}$ & MP & $\begin{array}{l}\text { Conductivity } \\
\text { (Btu/hr/ft) }\end{array}$ & $\begin{array}{c}\text { Specific } \\
\text { Power } \\
\text { (watts } / \mathrm{gm})\end{array}$ & Half - Lafe & $\begin{array}{r}\mathrm{gm} / \mathrm{K} \\
\text { Curie }\end{array}$ & $\begin{array}{l}\text { Us- } \\
\text { able } \\
\text { Mev } \\
\end{array}$ & Daughter & $\begin{array}{l}\text { Percent } \\
\text { Isotope }\end{array}$ & $\begin{array}{l}\text { Density } \\
(\mathrm{gm} / \mathrm{ec})\end{array}$ & $\begin{array}{l}\text { VP at } \\
\text { Atmosphere }\end{array}$ & $\begin{array}{l}\text { rempera- } \\
\text { ture }\end{array}$ & $\mathrm{BP}^{\circ} \mathrm{C}$ & Watts/ce & $\begin{array}{c}\text { Thermal } \\
\text { Coefficient } \\
\text { of Expansion } \\
\end{array}$ & $\begin{array}{l}\text { Solubility in } \\
\text { Water }\end{array}$ \\
\hline $\begin{array}{l}{ }^{*} \mathrm{Ce}-144 . \\
{ }^{*} \mathrm{Ce}_{2} \mathrm{O}_{3}\end{array}$ & $1321 \mathrm{~B}^{-}(218) \mathrm{Y}$ & $\begin{array}{l}144 \\
328\end{array}$ & $\begin{array}{l}804^{\circ} \mathrm{C} \\
1690\end{array}$ & 6.3 & $\begin{array}{l}(1.85) \\
(1.62)\end{array}$ & $285 \mathrm{D}$ & $\begin{array}{l}(0.584) \\
(0.785)\end{array}$ & 0.310 & $\operatorname{Pr}-144$ & & $\begin{array}{l}5.77 \\
6.8\end{array}$ & $6.5 \times 10^{-3}$ & $2000^{\circ} \mathrm{K}$ & $\begin{array}{l}2930^{\circ} \\
3300\end{array}$ & 20.8 & $5 \times 10^{-6}$ & \\
\hline $\mathrm{CeO}_{2}$ & & 172 & 1950 & $2.4^{(6)}$ & (1.54) & & $(0.697)$ & & $\begin{array}{l}17.5 \mathrm{~min} \\
\mathrm{Nd}-144\end{array}$ & & 7.3 & $1 \times 10^{-3}$ & & 3800 & $19.8(12.5)$ & & Insoluble \\
\hline $\mathrm{CeF}_{4}$ & & 216 & 977 & & $(1.25)$ & & $(0.885)$ & & $\begin{array}{l}2 \times 10^{15} \\
\text { years }\end{array}$ & & $\begin{array}{l}3.1 \\
\text { to } \\
4.7\end{array}$ & & & & & & \\
\hline $\mathrm{CeF}_{3}$ & & 197 & 1460 & & $(1.32)$ & & $(0.799)$ & & & & $\begin{array}{l}5.8 \\
\text { to } \\
6.1\end{array}$ & $6.5 \times 10^{-8}$ & & 2300 & & & Slighti $\gamma$ solubic \\
\hline $\mathrm{CeI}_{3}$ & & 520 & 752 & & 0.51 & & $\{2.11\}$ & & & & & & & 1390 & & & \\
\hline $\mathrm{CeCl}_{3}$ & & 246 & 802 & & $(1.06)$ & & $(0.995)$ & & & & 3.92 & 1 atmos & & 1730 & & & Very soluble \\
\hline $\mathrm{CeBr}_{3}$ & & 380 & 732 & & $(0.70)$ & & $(1.540)$ & & & & & & & 1550 & & & Very soluble \\
\hline $\mathrm{CeC}_{2}$ & & 168 & $>1000$ & & $(1.48)$ & & $(0.682)$ & & & & 5.23 & & & & & & Decomposes \\
\hline $\mathrm{CeB}_{6}$ & & 209 & 2190 & & $(1.20)$ & & $(0.847)$ & & & & & & & & & & \\
\hline $\begin{array}{l}\mathrm{CeS} \\
\mathrm{Ce}_{2}\left(\mathrm{MoO}_{4}\right)_{3}\end{array}$ & & $\begin{array}{l}171 \\
760\end{array}$ & 2500 & & $(1.56)$ & & $(0.692)$ & & & & $\begin{array}{l}5.93 \\
4.83\end{array}$ & & & & & & Insoluble \\
\hline $\mathrm{Ce}_{2}\left(\mathrm{WO}_{4}\right)_{3}$ & & 1024 & & & & & & & & & 10.89 & & & & & & Very singhtly soluble \\
\hline CeN & & 158 & 2000 & & $(1.68)$ & & & & & & & & & & & & \\
\hline$*_{\mathrm{Cm}-242}$ & $\mathrm{SF}, \mathrm{N}, 6.110 \alpha, \gamma$ & 242 & 950 & & 120 & $162.5 \mathrm{D}$ & 0.3018 & 0.025 & $\mathrm{Pu}-138$ & & 13.5 & & & & 1620 & & \\
\hline${ }^{*} \mathrm{Cm}_{2} \mathrm{O}_{3}$ & & & & & 109.2 & & & & $\begin{array}{l}86.4 \mathrm{X} \\
\mathrm{U}-234\end{array}$ & & $11.75^{0)}$ & & & & 1169 & & \\
\hline $\begin{array}{l}\mathrm{CmO}_{2} \\
{ }^{2} \mathrm{Cm}_{2} \mathrm{C}_{3}\end{array}$ & & 274 & 1500 & & 106 & & & & $\begin{array}{l}2 \times 10^{5} \\
\text { years }\end{array}$ & & $10.7^{(1)}$ & & & & 1245 & & Farrly soluble \\
\hline$* \mathrm{CmC}$ & & 254 & 2000 & & 114.3 & & 0.317 & & & & 10 & & & & 1143 & & \\
\hline${ }^{*} \mathrm{Cm} \quad 0.30 \mathrm{~N} 1$ & & & & & 14.8 & & & & & & $9.05^{(1)}$ & & & & 135 & & \\
\hline $\mathrm{CmF}_{3}$ & & & $\sim 500$ & & 97.2 & & & & & & $9.7^{(1)}$ & & & & 943 & & \\
\hline $\mathrm{CmCl}_{3}$ & & & $\sim 500$ & & 83.6 & & & & & & $5.8^{(1)}$ & & & & 488 & & 1 milligram/later $\mathrm{H}_{2} \mathrm{O}$ \\
\hline $\mathrm{CmBr}_{3}$ & & & -500 & & 60.3 & & & & & & $7.0^{(1)}$ & & & & 422 & & \\
\hline$A m-241$ & $5.475 \alpha, 0.060 \gamma . \mathrm{SF}$ & 241 & $1200^{\circ} \mathrm{C}$ & & 0.1176 & $458 \mathrm{Y}$ & 307.6 & 0.035 & $\mathrm{~Np}-237$ & & 11.7 & & & & & & \\
\hline $\mathrm{AmO}$ & & 257 & & & & & & & $2.2 \times 10^{6}$ & & & & & & & & \\
\hline $\mathrm{AmO}_{2}$ & & 273 & & & & & & & & & & & & & & & \\
\hline $\mathrm{Am}_{2} \mathrm{O}_{3}$ & & 530 & & & & & & & & & & & & & & & \\
\hline $\begin{array}{l}{ }^{*} \mathrm{Cs}-137 \\
\mathrm{CsCl}\end{array}$ & $0.529 B^{-} Q=1.2$ & $\begin{array}{l}137 \\
172.5\end{array}$ & $\begin{array}{l}28.5 \\
646\end{array}$ & & $\begin{array}{l}0.309 \\
0.249\end{array}$ & $33 \mathrm{Y}$ & 12.6 & 0.0529 & & 5.9 & $\begin{array}{l}1.87 \\
3.97\end{array}$ & & & $\begin{array}{r}670 \\
1292\end{array}$ & $\begin{array}{l}0.578 \\
0.99\end{array}$ & & \\
\hline $\mathrm{CsBr}_{3}$ & & 212 & 636 & & 0.079 & & & & & & 4.44 & & & 1300 & & & \\
\hline $\mathrm{CsF}$ & & 154.6 & 864 & & & & & & & & 3. 58 & & & 1250 & & & \\
\hline $\begin{array}{l}\mathrm{Cs}_{2} \mathrm{O}_{4} \\
\text { CsS }\end{array}$ & & 324 & 600 & & & & & & & & 3.77 & & & & & & \\
\hline $\left.\mathrm{Cs}_{2} \mathrm{~S}_{2}\right)$ & & 164 & 460 & 4.6 & & & & & & & & & & 800 & & & \\
\hline
\end{tabular}

(1) Extrapolated values
(2) Values obtanable on commerical materials
(3) Compacted and sintered sample

5) Computed from Wiedermann ratio for $p=44 \mathrm{w}-\mathrm{ohm}-\mathrm{cm}$

(6) Sintered sample

(7) $200 \mathrm{ps}$ forming pressure 


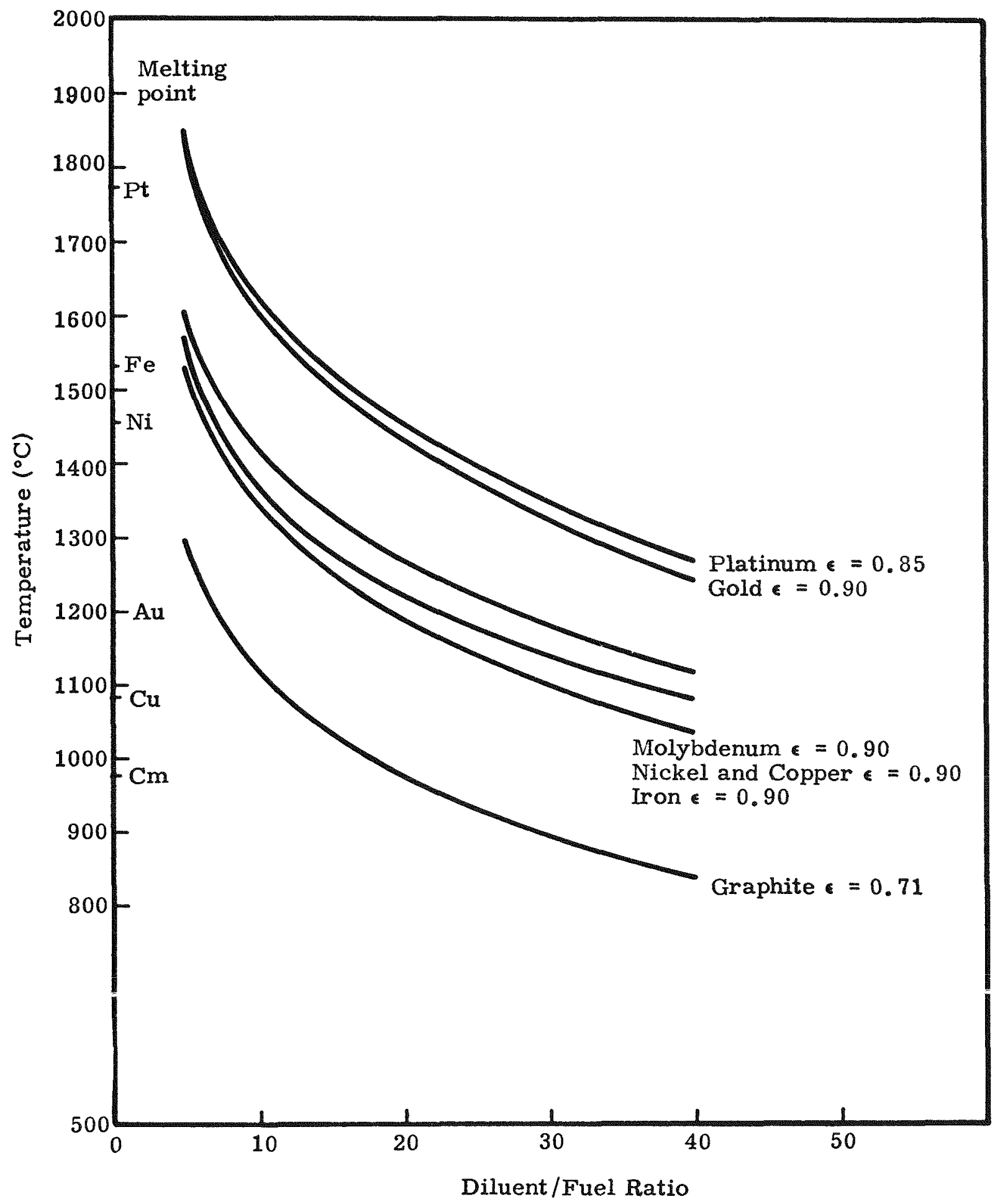

Fig. 98. Diluent/Fuel Ratio vs Surface Temperature 
(4) High power density, 0.5 to 10 watts/gram.

(5) Fuel burnup upon re-entry from orbital flight.

(6) High tensile strength and low creep properties at temperatures in the range of $1000^{\circ}$ to $2000^{\circ} \mathrm{F}$.

(7) Insolubility in fresh and sea water.

A graphite matrix has poor nuclear properties since it will moderate any neutrons emitted by the source. However, it is one of the better known ablative materials and has attractive conductivity and density so its use as a matrix material appears feasible.

Gold and its alloys melt at relatively low temperatures and are very heavy but have good thermal conductivities.

Although copper is weak in its nuclear and creep characteristics, the excellent conductivity and density of this metal make it a good prospect.

Iron will meet most of the property criteria, but its corrosion resistance is poor.

Nickel has properties similar to iron with the exception that its thermal conductivity is lower. It does, however, alloy with curium readily, making it very attractive as a fuel matrix material.

Molybdenum is in the moderate density range, has a high conductivity and no problem of burnup upon re-entry. However, the oxidization of pure molybdenum at temperatures above $1200^{\circ} \mathrm{F}$ is very rapid. In the event of a launch pad abort, containment of the fuel would be difficult unless provisions are made to jacket the capsule with a protective outer barrier.

Of the remaining materials studied, vanadium appears to be a possibility with its low density and somewhat above average thermal conductivity.

Figure 98 shows the surface temperature for several ratios of matrix materials mixed with curium. These curves present the worst case of a radiant boundary assuming no convection or conduction heat transfer. The fuel pellet has a design power of 1000 thermal watts and a length-to-diameter ratio of one for all mixtures. The relative emissivity of the platinum and graphite are experimental values, whereas those of the remaining material are obtained by assuming a surface coating of high emissivity. A sample calculation of the fuel pellet surface temperature for radiant boundary conditions is presented in Appendix F. The calculation is for a weight ratio of 20 parts nickel to 1 part curium by weight. 
TABLE 17

Fuel Matrix Materials for Cm-242 Compounds

\begin{tabular}{|c|c|c|c|c|}
\hline Material & $\begin{array}{l}\text { Melting } \\
\text { (०F) } \\
\end{array}$ & $\frac{\text { Point }}{\left({ }^{\circ} \mathrm{C}\right)}$ & $\begin{array}{l}\text { Thermal Conductivity } \\
\left(\mathrm{Btu} / \mathrm{hr}-\mathrm{ft}-{ }^{\circ} \mathrm{F}\right)\end{array}$ & $\begin{array}{l}\text { Density } \\
\text { (gm/cc) }\end{array}$ \\
\hline Molybdenum & 4760 & 2620 & 84.6 & 10.20 \\
\hline Rhodium & 3571 & 1985 & 50.0 & 12.50 \\
\hline Zirconium & 3355 & 1900 & $\begin{array}{l}15.5\left(1200^{\circ} \mathrm{C}\right)^{*} \\
9.6\end{array}$ & 6.40 \\
\hline Platinum & 3224 & 1773 & 42.0 & 21.37 \\
\hline Titanium & 3275 & 1800 & 8.1 & 4.50 \\
\hline Vanadium & 3100 & 1710 & $\begin{array}{l}17.9\left(100^{\circ} \mathrm{C}\right) \\
21.3\left(500^{\circ} \mathrm{C}\right)\end{array}$ & 5.96 \\
\hline Palladium & 2829 & 1553 & 41.0 & 12.16 \\
\hline Iron (pure) & 2795 & 1535 & $46.3\left(1200^{\circ} \mathrm{C}\right)$ & 7.85 \\
\hline Mo, $\mathrm{Ni}_{1} \mathrm{Cr}$ steel & & 2735 & 1500 & 21.7 \\
\hline Nickel & 2620 & 1455 & $\begin{array}{l}35\left(100^{\circ} \mathrm{C}\right) \\
14\left(1200^{\circ} \mathrm{C}\right)\end{array}$ & 8.90 \\
\hline Copper & 1960 & 1083 & 210 & 8.94 \\
\hline Gold & 1945 & 1063 & 170 & 19.32 \\
\hline Graphite & 6422 & 3550 & 59 to 78.8 & 2.25 \\
\hline
\end{tabular}

B. PROCESS SELECTION

1. Americium

During this period most of the effort was directed toward an evaluation of available processes for the preparation of curium starting with an Americium-241 solution. At the present time, the Government has sufficient americium available to purify 100 grams for irradiation. It is known that americium as received from the Dow Chemical Company's 
Rocky Flats Plant has less than 1\% plutonium (Ref. 27)。A purification process has been developed at the University of California for producing high-grade americium in the form of the oxide. Details of this process will be described under Subtask 6.2; however, of all the methods of purifying, this is the most highly developed. Some chemical handling problems still exist, especially in the ion exchange separation steps.

Following an outline proposed in a previous quarterly report (Ref. 34 ), the americium oxide $\left(\mathrm{AmO}_{2}\right)$ powder is blended with very fine mesh, high purity aluminum powder in about a 30/1 ratio of aluminum to americium oxide (calculated as the metal). When the americiumaluminum pellets are compacted, $95 \%$ of theoretical density is achieved provided sufficient pressure is applied to the rams of the press. These pellets are then inserted in aluminum tubes, capped and fusion welded. This phase of the process offers no problems. After weiding, the slugs are shipped to a facility such as the Materials Testing Reactor for irradiation in a high neutron flux. After an 84-day irradiation period, about $43 \%$ of the americium is converted to Curium-242 (Ref. 27).

\section{Curium}

Several methods of purifying have been suggested. One method was developed at the University of California Radiation Laboratories at Livermore, California (Ref. 35), and is described in U. S. Patent No. 2,887,358 dated May 19, 1959, by G. H. Higgens, et al. By this procedure, curium is isolated as the oxide along with the unconverted americium oxide. A second method has been developed by Atomic Energy of Canada, Limited, at the Chalk River Project. Details of this process will be analyzed during the next period to determine whether americium can be further isolated from curium to permit recycling of the unconverted americium.

\section{Curium Conversions}

After purification of the curium, processes for converting the material to the desired fuel compound must be developed. Some information is available in the literature for converting curium oxide to carbides.

A method for reducing curium to the metal has been reported by the University of California Radiation Laboratory in Livermore, California, but the process has not been fully developed (Ref. 35). This reduction requires the conversion of curium to the trivalent fluoride and then reducing the fluoride to the metal with either calcium or barium metal. Careful control must be maintained to be sure the curium metal will separate from the fluoride slag. For this reduction 
step, it is extremely important that all constituents are absolutely dry, otherwise hydrogen will be generated, forming an explosive mixture with air, or reacting violently with the oxide in the americium and aluminum.

\section{Plutonium Conversions}

Similar reductions have been reported on plutonium. Information on these processes is available in the classified literature. Studies of the reduction processes are scheduled for the next period.

\section{Fuel Forms}

Studies of the various matrix alloys with curium have been studied and will be discussed under Subtask 6.3. For fuels being considered for space missions, $87 \%$ of the selected fuel form must be burned up to less than 5 microns. Such materials as gold, nickel, molybdenum and iron meet this requirement, but the capsules must also resist corrosion in both land and sea environment after impacting on the earth's surface (Ref. 26). Most of the super alloys (Ref. 26) are satisfactory for corrosion resistance and abort conditions, but methods for burnup to the desired size must be devised in order to meet the Commission's requirements. Plasma jet tests will be conducted under Task 6.3 to determine the suitability of these container materials. 


\section{SUBTASK 6.2--AMERICIUM AND CURIUM RADIOISOTOPE PREPARATION PROCESSES}

\section{A. AMERICIUM PURIFICATION AND CONVERSION}

A modified process for the purification of gram quantities of americium by means of ion exchange resins is being developed by The Martin Company based on a procedure used by the University of California. Americium chloride dissolved in $6 \mathrm{~N}$ hydrochloric acid as received from Rocky Flats Laboratory will be processed to reduce the plutonium content to less than $0.01 \%$. An apparatus for purifying americium has been ordered from Dublin Industries. This equipment has been designed to handle, with a minimum of hand operations, gram quantities of any transuranic element having low energy gamma rays (see Fig.99).

After purification, the americium is converted to the oxalate, and dried. The oxalate is then decomposed to americium oxide by heating in a furnace. This material is to be used in conjunction with americium fuel slug fabrication.

\section{B. AMERICIUM SLUG FABRICATION}

In preparation for fabricating fuel slugs for irradiation of americium, the oxide is blended with pure aluminum powder for compacting into pellets. A dry box and die assembly was procured during the latter part of September, and work was started on the assembly and checkout of the equipment and process variables. The dry box was designed to hold a die assembly (see Fig.100) and fit a modified 50-ton, Denison automatic powder press, Model NA-50-C95. It is planned that, with this equipment, a dispersion of americium oxide and aluminum powders will be compacted into pellets approximately $7 / 8$ of an inch in diameter by 1 inch long. The green compacts will then be loaded into an aluminum tube 5 inches in length and 1 inch in diameter. The pellets will then be coined and the tube sealed by the heliarc weld process. Figure 101 shows a finished dummy slug and its components. Approximately 14 of these slugs will be required around the middle of 1960 for the first irradiation cycle.

Studies of the powder metallurgy characteristics of the 4 wt $\%$ rare earth oxide $\left(R_{\circ} E_{0}\right)$ and aluminum (Al) mixture were initiated to determine the effect of particle size, type lubricant, compacting motion and pressure on the density of green compacts. However, early in this investigation, after fabrication of a few pellets, galling of the punches became very pronounced. After investigating the causes of the galling, 


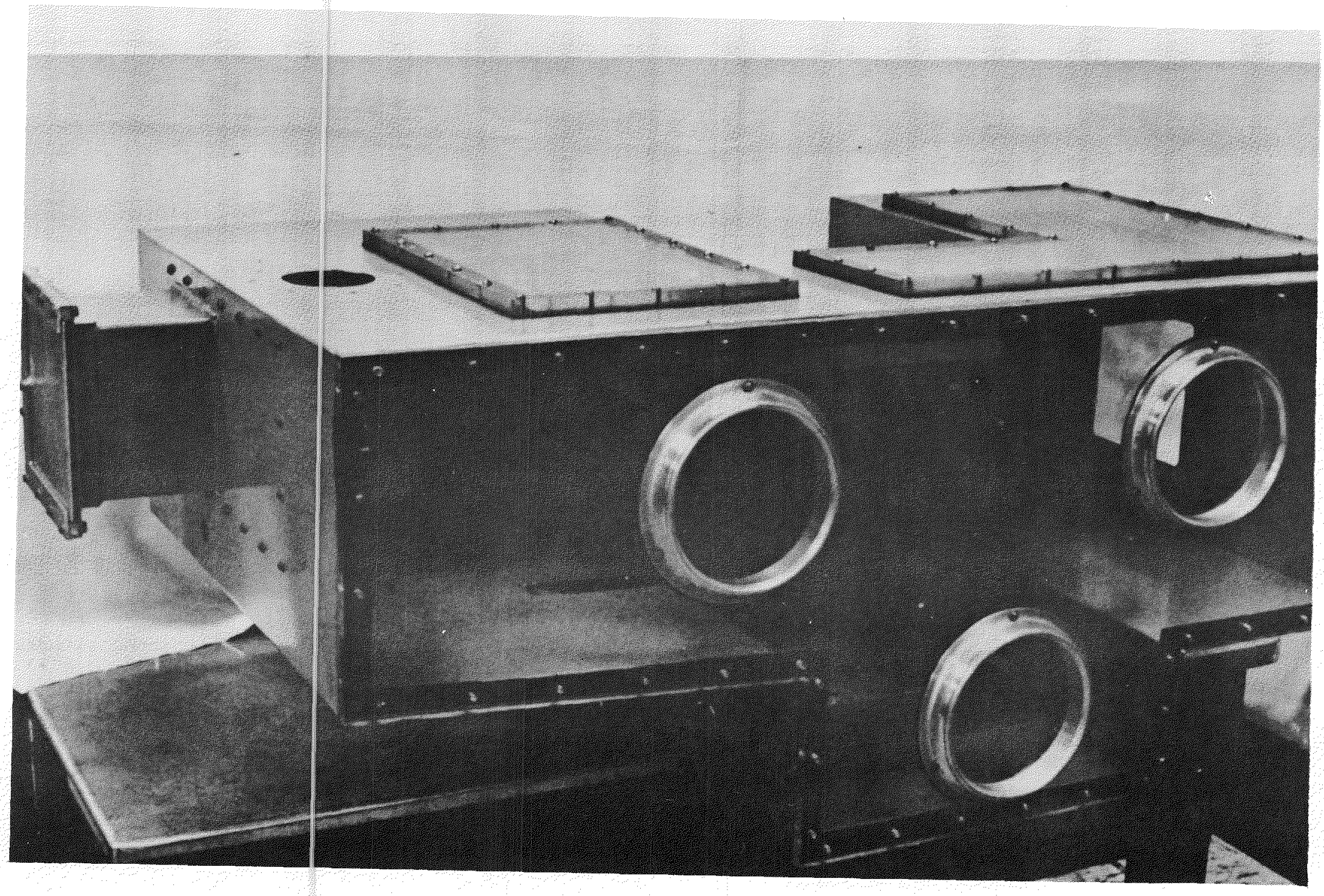

Fig. 99. Compacting Press Dry Box 


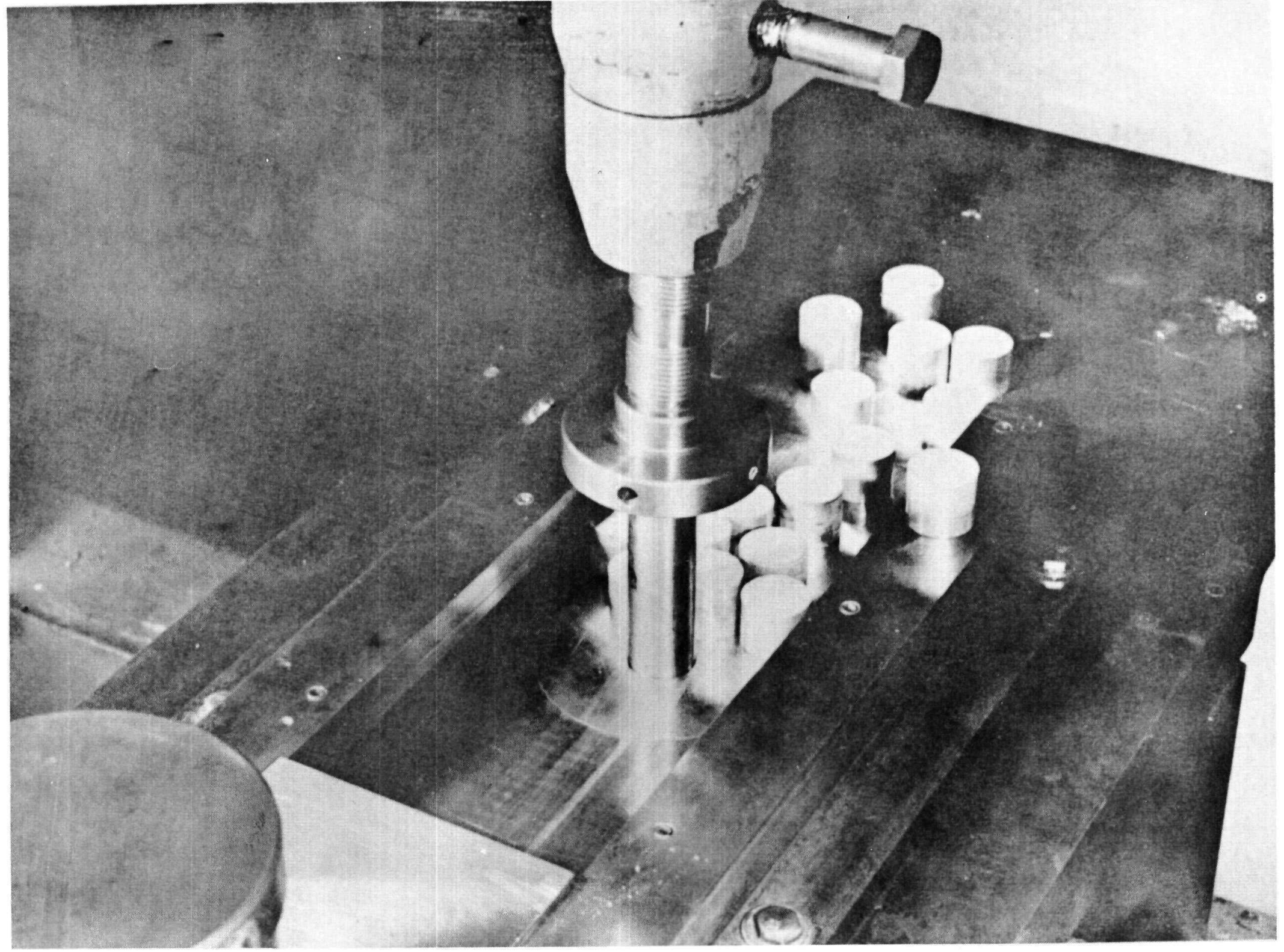

Fig. 100. Americium Fuel Compacting Feed Mechanism and Die Assembly 


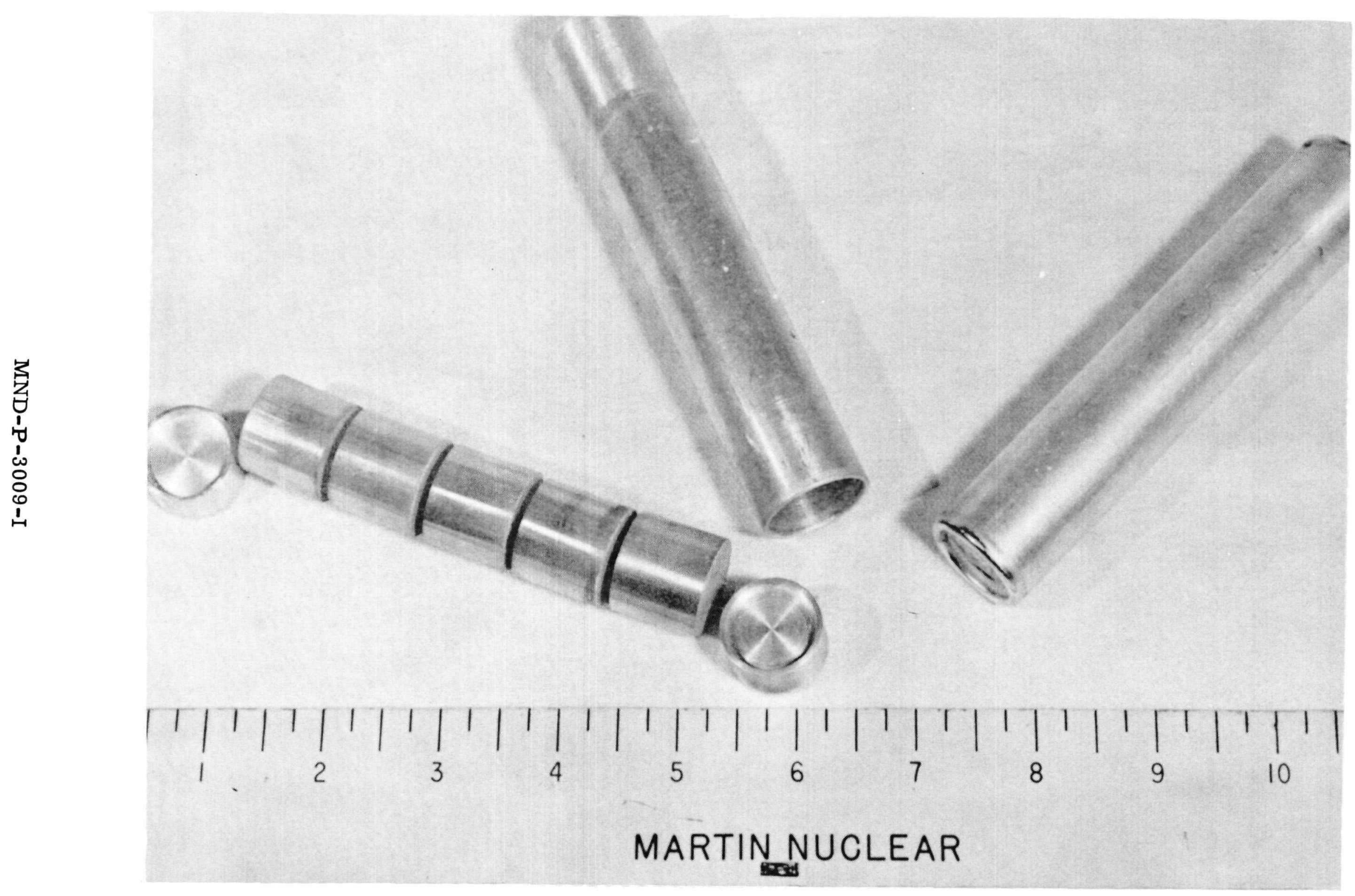

Fig. 101. Americium Aluminum Fuel Fellets and Container 
replacement of the brass tips with hardened tool steel die tips eliminated all wear on the dies and tips. With the new hardened dies, a number of pellets were fabricated and their densities measured in order to determine the effects of the powder metallurgy variables (see Table 18). From the results of these measurements and observations made during this study, the following information was obtained:

(1) Pellet densities were proportional to the compacting pressure; the higher the pressure, the higher the density. This can be seen in Fig. 102 .

(2) A study of different type lubricants indicated no specific advantage of any one over the others. Graphite, Molykote, stearic acid and graphite-Molykote lubricants blended into the powders were tried.

(3) Density varied irregularly with particle size. The highest densities were obtained from 100\% -325 mesh powders (see Fig, 103).

(4) Compacts made using double-action motion had slightly higher densities than those made by single action. In addition, due to more uniform density gradients, the double-action compacts had better handling characteristics.

(5) There appeared to be no major problems in compacting the aluminum-4 wt \% rare earth oxide powders.

(6) Some redesign of the die assembly is required. Areas to be considered for redesign are as follows:

(a) Materials of construction for the powder reservoir since the present brass hopper galled severely after fabricating only a few pellets.

(b) Materials of construction and design of punches to permit higher loads without distortion of the bottom punch.

(c) Interchangeable parts for compacting and coining in the same dry box assembly to reduce the processing and handling time.

(d) Improved tolerances on die punches and body with regard to dismantling in the dry box. 
TABLE 18

Al $+4.2 \%$ Rare Earth Oxide--Green Compact Data

\begin{tabular}{|c|c|c|c|c|c|c|}
\hline $\begin{array}{c}\text { Compacting } \\
\text { Pressure } \\
\text { (tsi)* } \\
\end{array}$ & $\begin{array}{l}\text { Particle* } \\
\text { Size } \\
\end{array}$ & $\begin{array}{c}\text { Compacting } \\
\text { Action } \\
\end{array}$ & $\begin{array}{c}\text { Lubricant } \\
\text { Used** }\end{array}$ & $\begin{array}{l}\text { Average } \\
\text { Density } \\
\text { (gm/cc) }\end{array}$ & $\begin{array}{c}\text { Average } \\
\text { Height } \\
\text { (inches) }\end{array}$ & $\begin{array}{r}\text { Actual To } \\
\text { Theoretical } \\
\text { Density Rati }\end{array}$ \\
\hline 3.3 & A & Single & $S$ & 2.11 & 0.82 & 0.756 \\
\hline 3.3 & A & Single & $G+M$ & 2.11 & 0.77 & 0.756 \\
\hline 5.0 & A & Single & $\mathbf{M}$ & 2.155 & 0.79 & 0.770 \\
\hline 5.0 & A & Single & $G+M$ & 2.16 & 0.74 & 0.775 \\
\hline 5.0 & B & Single & $\mathbf{M}$ & 2.16 & 0.75 & 0.775 \\
\hline 5.0 & C & Single & S & 2.13 & 0.79 & 0.763 \\
\hline 5.0 & D & Single & $G$ & 2.10 & 0.75 & 0.750 \\
\hline 5.0 & $E$ & Single & $G+M$ & 2.14 & 0.75 & 0.766 \\
\hline 8.3 & A & Single & $\mathrm{s}$ & 2.32 & 0.68 & 0.830 \\
\hline 8.3 & A & Single & $\mathrm{G}+\mathrm{M}$ & 2.36 & 0.77 & 0.847 \\
\hline 3.3 & A & Double & $\mathrm{S}$ & 2.13 & 0.83 & 0.763 \\
\hline 3.3 & A & Double & $\mathrm{G}+\mathrm{M}$ & 2.125 & 0.75 & 0.763 \\
\hline 5.0 & A & Double & $\mathbf{M}$ & 2.205 & 0.77 & 0.790 \\
\hline 5.0 & A & Double & $\mathrm{G}+\mathrm{M}$ & 2.215 & 0.72 & 0.795 \\
\hline 5.0 & B & Double & $\mathbf{M}$ & 2.18 & 0.74 & 0.782 \\
\hline 5.0 & $\mathrm{C}$ & Double & $S$ & 2.13 & 0.75 & 0.763 \\
\hline 5.0 & $\mathrm{D}$ & Double & G & 2.15 & 0.72 & 0.770 \\
\hline 5.0 & $\mathbf{E}$ & Double & $G+M$ & 2.17 & 0.71 & 0.780 \\
\hline 8.3 & A & Double & $\mathrm{G}$ & 2.36 & 0.70 & 0.847 \\
\hline 8.3 & A & Double & $G+M$ & 2.36 & 0.69 & 0.847 \\
\hline 9.96 & B & Double & $\mathbf{M}$ & 2.405 & 0.63 & 0.865 \\
\hline 9.96 & $\mathrm{C}$ & Double & $\mathrm{S}$ & 2.42 & 0.65 & 0.869 \\
\hline 9.96 & $D$ & Double & $G$ & 2.415 & 0.62 & 0.869 \\
\hline 9.96 & $\mathbf{E}$ & Double & $G+M$ & 2.42 & 0.59 & 0.869 \\
\hline 14.94 & $\mathrm{C}$ & Double & $s$ & 2.525 & 0.62 & 0.905 \\
\hline
\end{tabular}

*Al particle size key:

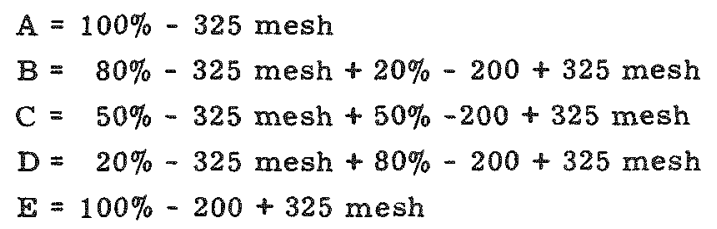

** Lubricant key:

$\mathrm{G}=$ Graphite, 0.5 wt $\%$ blended in powder

$\mathrm{S} \quad=$ Stearic acid, $0.5 \mathrm{wt} \%$ blended in powder

$\mathrm{M}=$ Molykote (microsize, $0.5 \mathrm{wt} \%$ blended in powder

$\mathrm{G}+\mathrm{M}=0.25 \%$ Graphite plus $0.25 \%$ Molykote blended in powders 


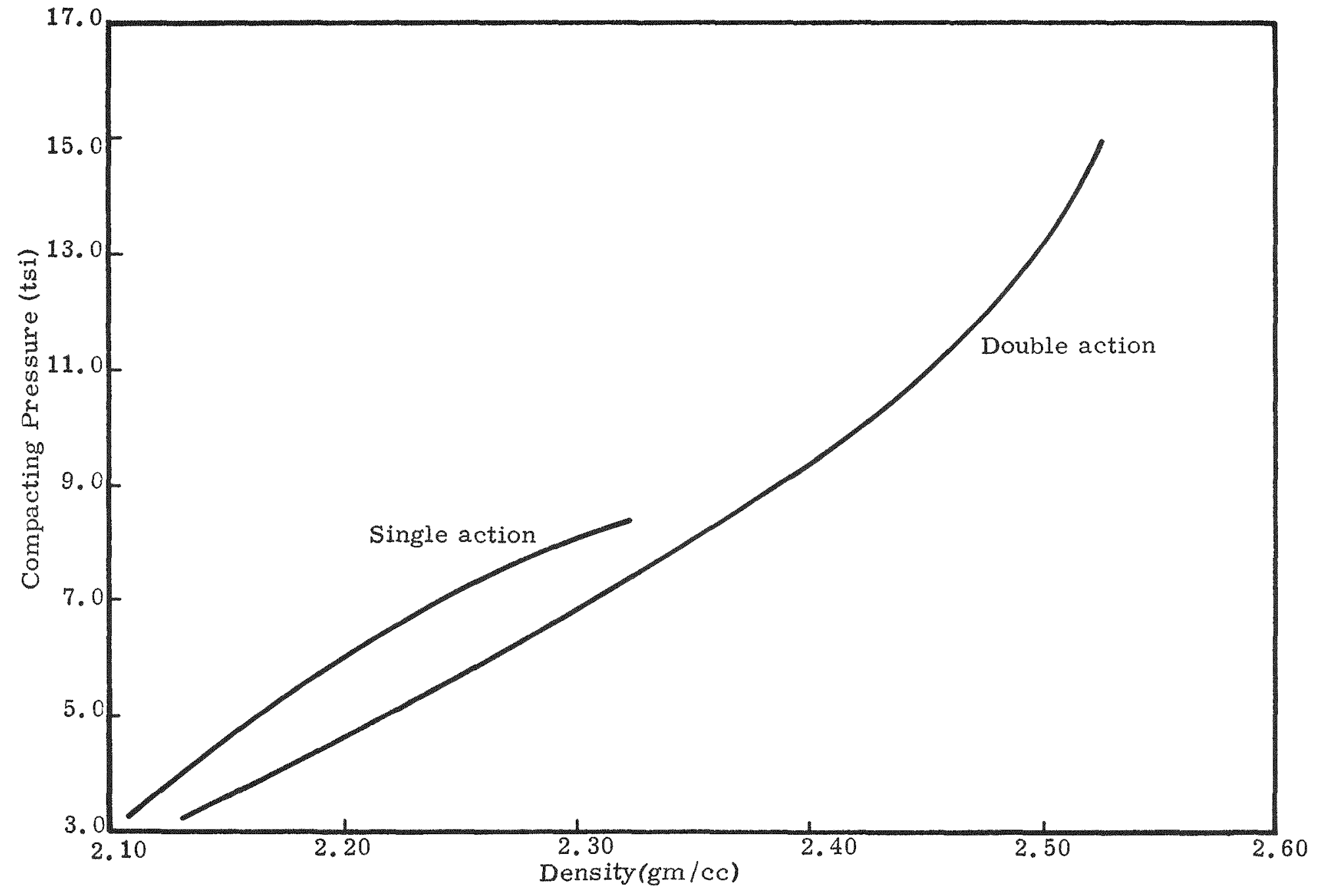

Fig. 102. Aluminum Fellet Compacts Pressure vs Density 


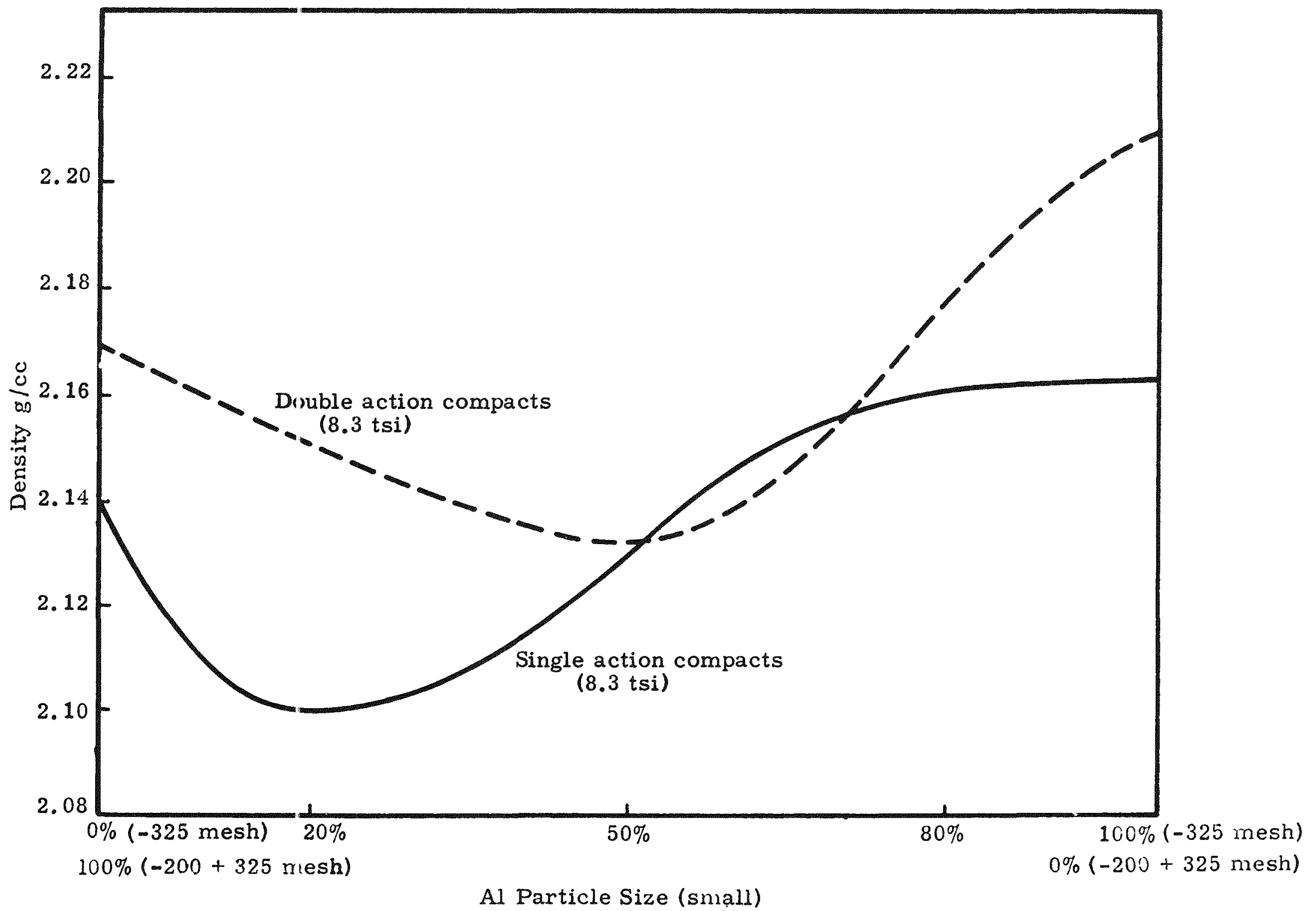

Fig. 103. Effect of Aluminum Particle Size on Compaction Density 


\section{PURIFICATION OF CURIUM}

One of the objectives of the task is to develop methods for separating kilocurie quantities of curium from the fission products and other impurities generated during the 84 -day irradiation in the high intensity neutron flux.

Investigations were conducted on solvent extraction systems to determine the feasibility of removing the curium in a concentrated form without ex tracting the fission products and impurities. Also under consideration is the use of ion exchange resins which preferentially absorb ions of americium-curium while eliminating the fission products in the effluent.

\section{Solvent Extractions}

Preliminary solvent extraction procedures were tried using gadolinium to simulate curium. The following solvents were tested: thenoyl trifluoroacetone (TTA) in xylene and in benzene; diethylhexylorthophosphoric acid (HDEHP) in heptane, in toluene and in $\mathrm{CC}_{4}$; tri-n-butyl phosphate (TBP) in toluene and in $\mathrm{CC}_{4}$; and acetylacetone and ethyl acetone. Although all have favorable decontamination ratios, the most promising for extracting gadolinium but not other rare earths or fission products was HDEHP in toluene and in $\mathrm{CC}_{4}$. Since toluene is a volatile flammable solvent, the nonflammable carbon tetrachloride system will be considered in future studies. It must be emphasized that, although the lanthanide, gadolinium, is very similar chemically to the corresponding actinide, curium, it is very possible that any procedures developed with gadolinium may not extract in precisely the same manner when curium is present.

Experiments with radioactive tracers. Some radioactive rare earth tracers, obtained from work done under the strontium purification program, were used to conduct some ion exchange separations. These tracers when mixed with a gadolinium solution produced an activity of 156,800 counts per minutes $(\mathrm{c} / \mathrm{m})$.

Diethylhexylphosphoric acid extractions. An extraction of the aqueous phase with $1.5 \mathrm{M}$ diethylhexylphosphoric acid (HDEHP) in carbon tetrachloride $\left(\mathrm{CC}_{4}\right)$ resulted in an activity of only $3800 \mathrm{c} / \mathrm{m}$ in the solvent phase, whereas essentially all of the gadolinium was extracted. This method produced a fission product decontamination or separation factor of about 40 by a single solvent extraction. It should be noted that the solvent phase had extracted primarily only short-lived fission products. The longer-lived isotopes remain in the aqueous phase.

Tributyl phosphate extractions. Concentrated tributyl phosphate was also tested on an aqueous solution containing gadolinium and fission 
products of the same level of activity. The aqueous solution had a concentration of $0.5 \mathrm{M}$ nitric acid. After extraction with tributyl phosphate, the solvent phase contained only a small amount of fission product $(2500 \mathrm{c} / \mathrm{m})$.

Depending upon the fission products present in the irradiated aluminum-americium slugs, one or both of these two systems may be suitable to decontaminate the curium.

Thenoyl trifluoroacetone extraction. Another system under test was thenoyl trifluoroacetone (TTA) in benzene on an aqueous solution of fission products ( $\mathrm{pH} 4$ ). According to work of Magnusson and Anderson, trace amounts of curium can be extracted by the system (Ref. 36).

Although gadolinium is not extracted by this system, there is evidence that curium, even in large quantities, would be separated if the system were employed.

\section{Ion Exchange Systems}

Preliminary investigations with ion exchange resin separations were conducted during this period. Dowex $A-1$ resin with an iminodiacetate active group has very high adsorptive properties for cations. A mixture of gadolinium and a radioactive isotope was placed on the column. After washing of the resin, the absorbed radioisotope was eluted with a $10 \%$ citric acid solution; however, the rate of elution was slow. The lowering of the citric acid concentration to $0.2 \%$ appreciably increased the amount of isotope eluted. No gadolinium was found in the eluted solution.

Similar investigations will be required to select the optimum elutrient solution conditions for americium and curium.

All work conducted during this period is considered exploratory in nature. Americium and curium must be used for confirmation of the ion exchange separation procedures. The experiments with small amounts of curium can be conducted in a shield cave of the type shown in Fig. 104. For kilocurie quantities, all experiments will have to be conducted in The Martin Nuclear Laboratories (R'ef. 13).

\section{ALUMINUM VOLATILIZATION PROCESS}

After the americium-aluminum slugs described in the previous section are irradiated in the Materials Test Reactor (or similar

facility) for a period of 90 days at a flux of $4 \times 10^{14} \mathrm{n} / \mathrm{cm}^{2} / \mathrm{sec}$, the slugs are removed from the reactor and allowed to cool for about one month before handling. The cool-down period is required to permit decay of the short-lived fission product and to allow the radiation dose to drop to a workable level. 


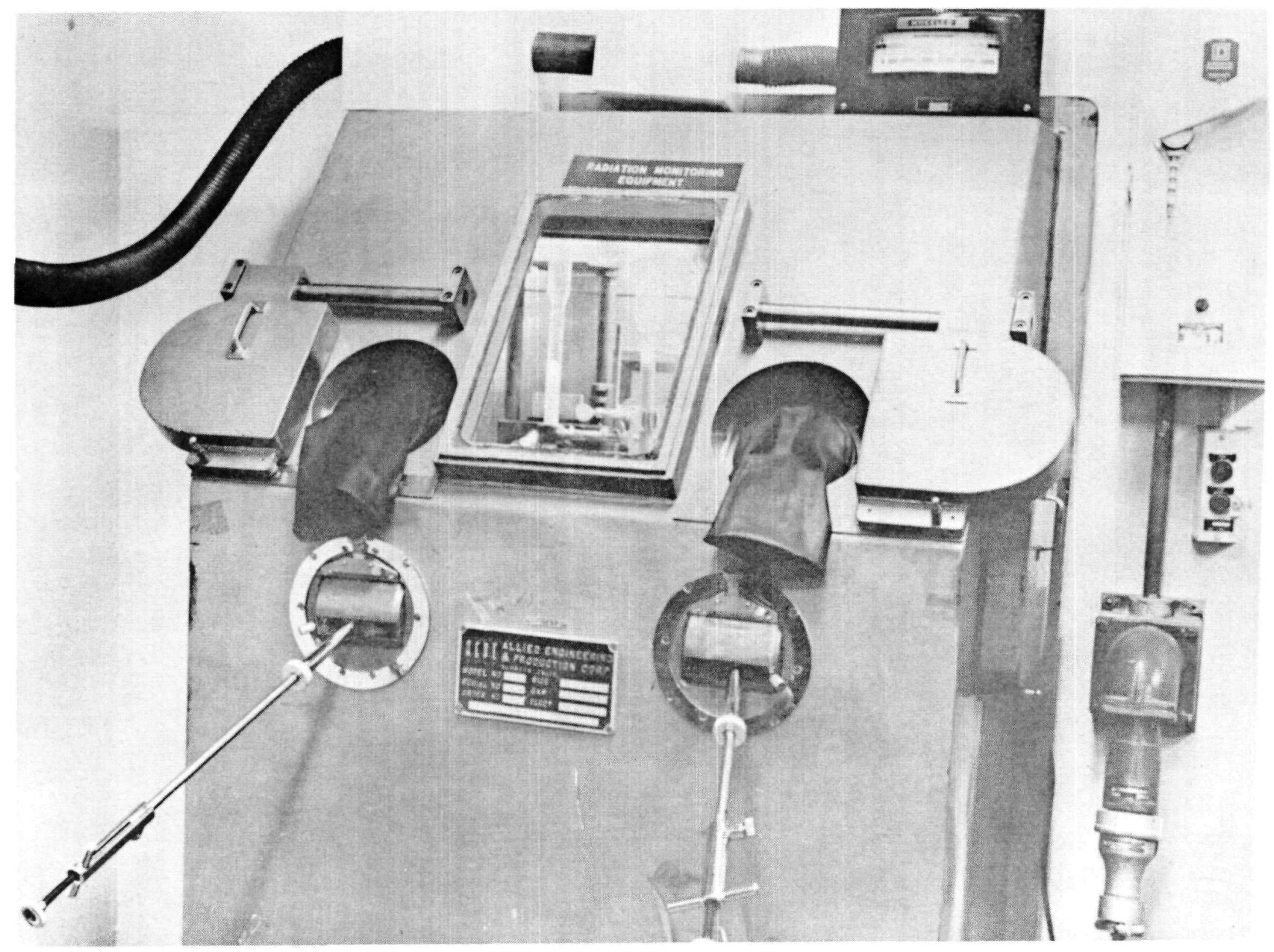

Fig. 104. Shielded Dry Box For Ion Exchange Purifications 
The converted fuel, Curium-242, must first be isolated from the aluminum before any removal of plutonium and other radioisotopes can be accomplished.

A process has been developed to volatilize the aluminum from the radioisotopes by passing dry chlorine gas diluted with argon over the aluminum slug. By maintaining the temperature in the furnace slightly below the temperature of the melting point of aluminum and controlling the flow rates of argon and chlorine, the aluminum is sublimed within a few hours, leaving only curium, plutonium, americium and nonvolatile fission products.

During this period, the fabrication of components for an experimental volatilization apparatus was completed, the system assembled and tested. Experimental runs were made on nonradioactive materials in an effort to determine problem areas concerning the reaction of aluminum with chlorine and the subsequent distillation of the aluminum chloride formed. The purpose of this investigation was the development of a process for the efficient separation of aluminum from the products yielded by the irradiation of americium oxide.

The principle components of the apparatus for the formation and sublimation of $\mathrm{AlCl}_{3}$ consisted of a gas inlet train, reaction vessel, condenser and gas absorption train. Figure 105 is a schematic of the system, and Figs. 106 and 107 show external and internal views of the equipment, respectively.

The operation of the system consists essentially of the following steps:

(1) Purge the system with an inert gas (argon).

(2) Preheat the sample to $600^{\circ} \mathrm{C}\left(1112^{\circ} \mathrm{F}\right)$.

(3) Pass a metered quantity of chlorine at a controlled rate over the sample using argon as a diluent.

(4) Monitor the reaction and control the rate of reaction through control of the furnace temperature and chlorine flow.

(5) Purge and cool the system with an inert gas.

(6) Discharge the reaction boat and condenser products.

A total of eleven experimental runs (the most significant are listed in Table 19) were made in the apparatus. Several problems were encountered. The first and probably most serious problem was with secondary reactions of chlorine or aqueous impurities with the structural material (stainless steel). Modifications to the furnace 


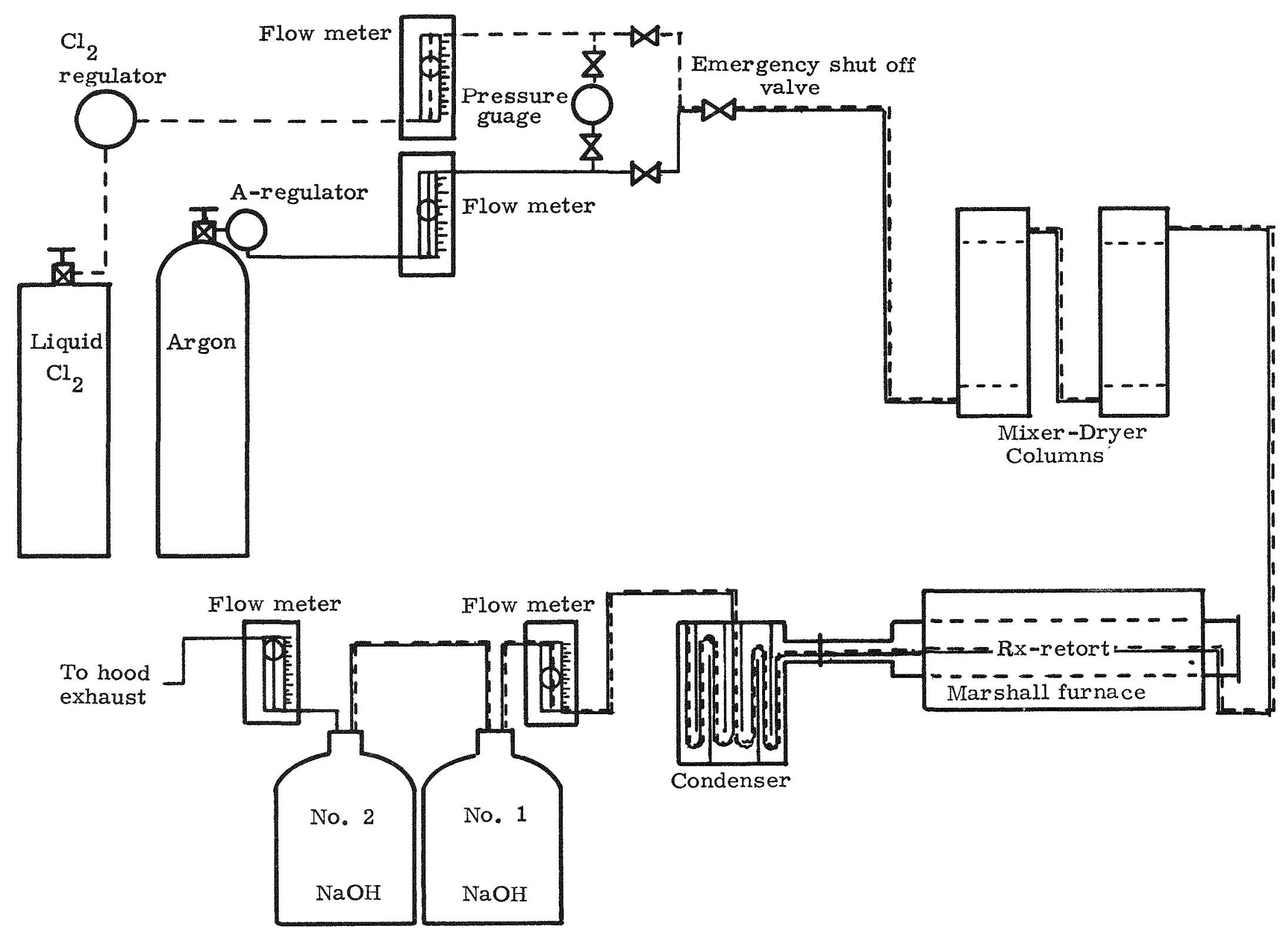

Fig. 105. Aluminum Distillation System 


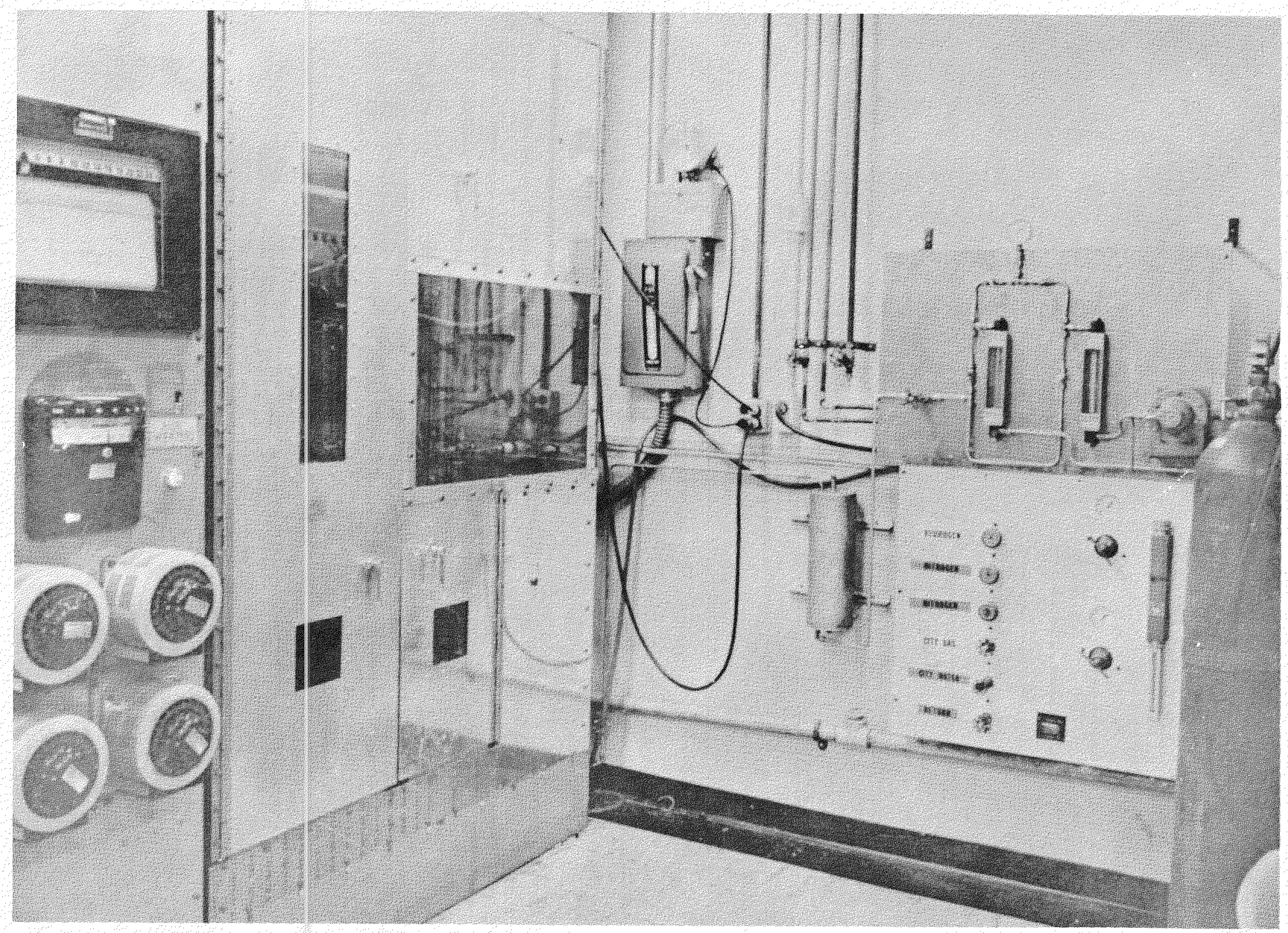

Fig. 106. Aluminum Volatilization Equipment (Exterior) 


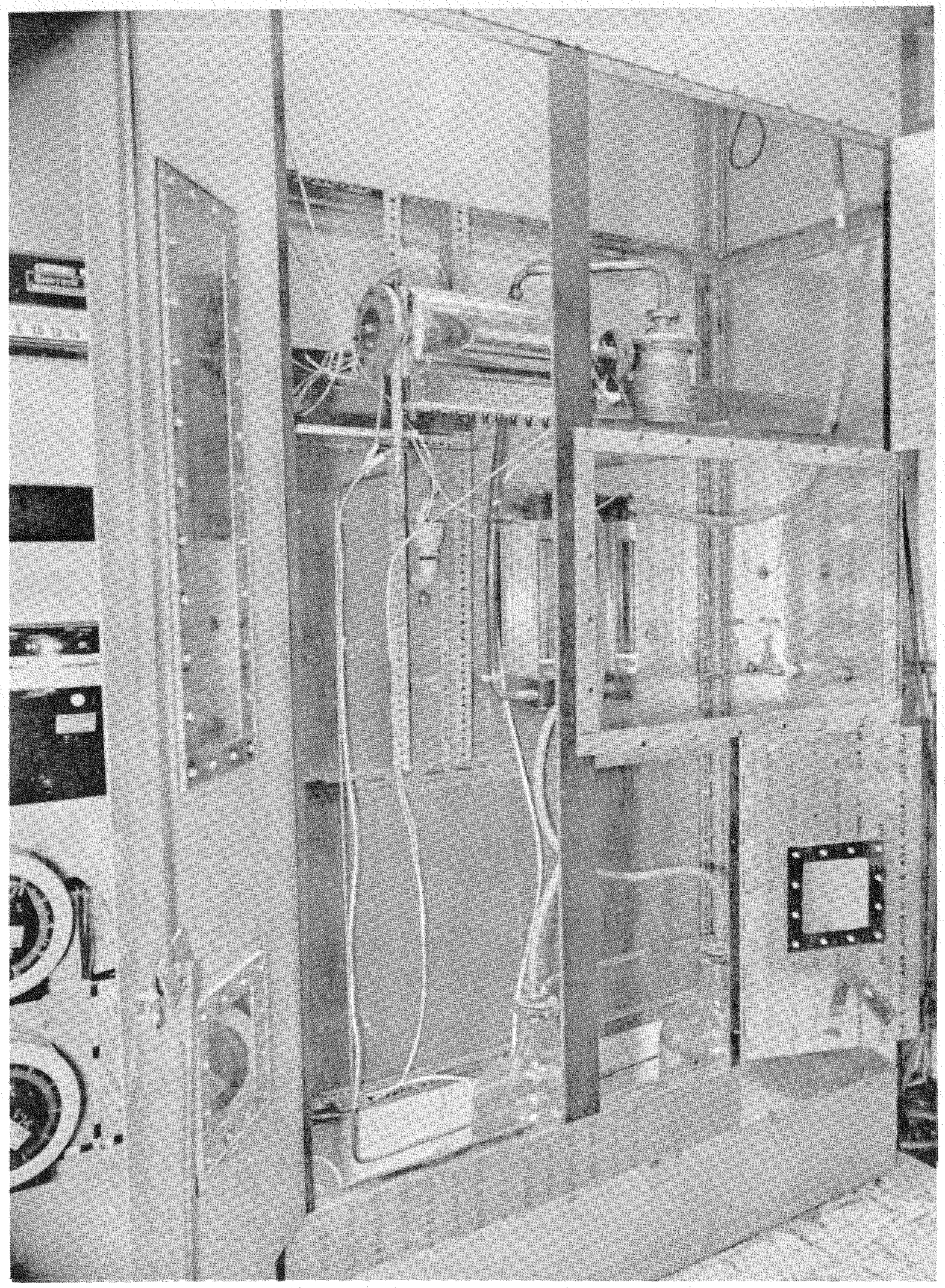

Fig. 107. Internal View of Aluminum Distillation Equipment 
TABLE 19

Aluminum Chloride Vaporization

\begin{tabular}{|c|c|c|c|c|c|}
\hline $\begin{array}{l}\text { Length } \\
\text { of Run } \\
\text { (hr) } \\
\end{array}$ & $\begin{array}{c}\text { Maximum Slug } \\
\text { 'Temperature } \\
\left({ }^{\circ} \mathrm{F}\right) \\
\end{array}$ & $\begin{array}{c}\text { Chlorine Flow } \\
\text { Rate } \\
\text { (cfh) } \\
\end{array}$ & $\begin{array}{c}\text { Argon Flow } \\
\text { Rate } \\
\text { (cfh) } \\
\end{array}$ & $\begin{array}{l}\text { Aluminum }+ \\
\text { Reactor Rate } \\
(\mathrm{gm} / \mathrm{hr}) \\
\end{array}$ & Remarks \\
\hline 0.75 & 1460 & 10 & 5 to 10 & 2.42 & $\begin{array}{l}\text { Slug melted-- Exit } \\
\text { tube plugged }\end{array}$ \\
\hline 0.5 & 1200 & 10 to 5 & 5 & 3.35 & $\begin{array}{l}\text { Controlled reactor } \\
\text { temperature } \\
\text { maintained }\end{array}$ \\
\hline 0.5 & 1155 & 7.5 & 5 & 2.68 & $\begin{array}{l}\text { Nos. } 2 \text { and } 3 \text { ther } \\
\text { mocouple out of } \\
\text { order }\end{array}$ \\
\hline 1.0 & 1193 & 7.5 & 5 & 2.47 & $\begin{array}{l}\text { Nos. } 2 \text { and } 3 \text { ther- } \\
\text { mocouple out of } \\
\text { order }\end{array}$ \\
\hline 2.1 & 1152 & 7.5 & 5 & 1.99 & $\begin{array}{l}\text { Heavy black oxide } \\
\text { coating on slug }\end{array}$ \\
\hline 4.1 & 1152 & 7.5 to 25 & 5 to 20 & 2.82 & $\begin{array}{l}\text { All chlorine re- } \\
\text { acted }\end{array}$ \\
\hline
\end{tabular}

KEY :

$\begin{aligned} \mathrm{cfh} & =\text { cubio feet per hour } \\ \mathrm{gm} / \mathrm{hr} & =\text { grams per hour }\end{aligned}$ 
are underway to install a Vycor tube with tantalum fittings for the rest of the system in the heat-affected zone. Another difficulty encountered was the leakage of high temperature silicone rubber gaskets resulting from their thermal deterioration. This was remedied by incorporating metal $\mathrm{O}$-ring seals into the system.

Another critical factor in the distillation of aluminum was found during an attempt to obtain a controllable rate of reaction of the aluminum with the chlorine. An aluminum sample melted down due to a sudden temperature excursion at the initiation of reaction and all means to control, short of cutting off the chlorine supply, failed. Conversion of the chlorine flowmeter to an expanded low range scale ( 0 to $10 \mathrm{cubic}$ feet per hour (cfh), instead of 0 to $30 \mathrm{cfh}$ ) and the modification of the recorder to reduce the response time for temperature sensing from two minutes to thirty seconds should aid greatly in solving this problem. Also, as a result of the aluminum melt down, it was found that a cold spot had developed on the exit end of the reaction vessel and caused plugging of the condenser inlet tube. This condensation was due to the controlling thermocouple being located near the center of the exothermic reaction. The relocation of the controlling thermocouple near the exit end of the reaction tube eliminated the cold spot.

During the volatilization of the aluminum pellets heavy black film coated the surface, which inhibited the formation of aluminum chloride $\left(\mathrm{AlCl}_{3}\right)$. Figures 108 and 109 shows the specimen immediately after its removal from the reaction chamber. The coating is very uniform, abrasive resistant and chemically inert and in many ways resembles oxide coatings obtained from the Martin "Hard Coat" process. The film composition and the source of contamination are not known at this time but will be the subject of analysis to determine how they can be avoided. 


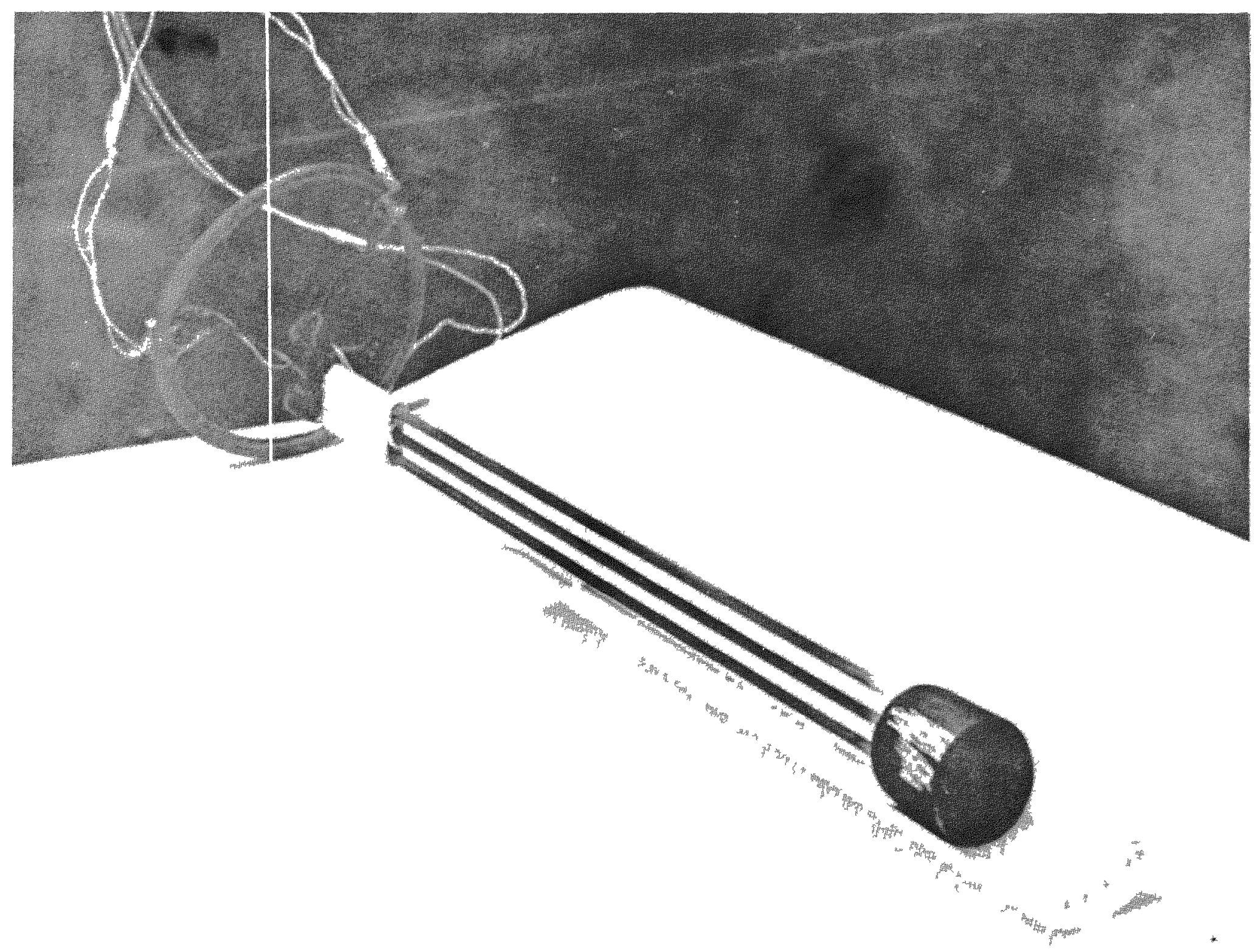

F1g. 108. Aluminum Pellets After Partial Volatilization 


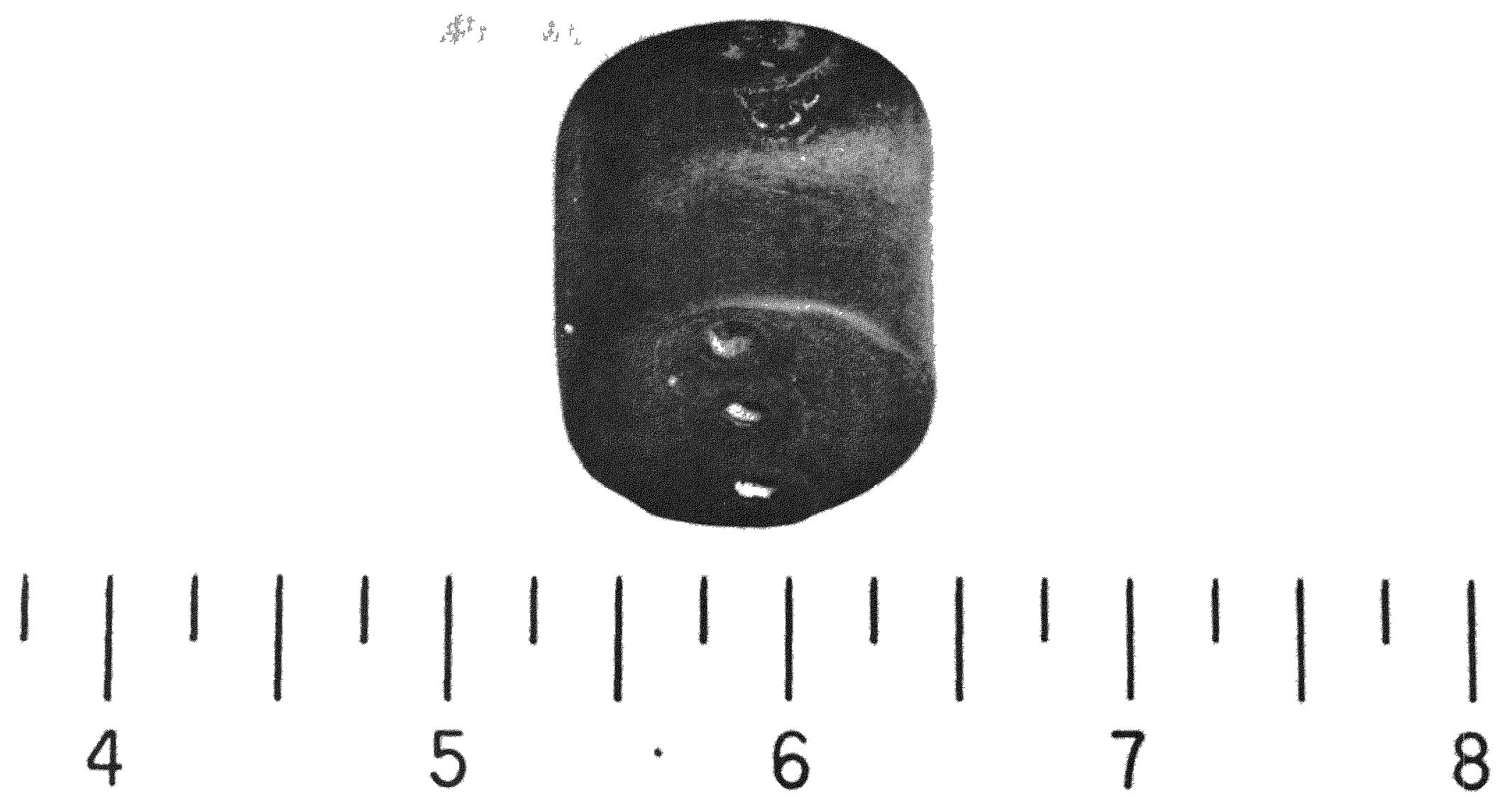

MARTIN NUCLEAR

Fig. 109. Coating on Aluminum Pellets After Partial Volatilization 
Blank page 


\section{SUBTASK 6.3--FUEL FORM}

No appreciable effort has been spent on the development of specific fuel forms under this task to date. Experimental investigations, analysis and evaluations will be conducted for curium and plutoniun fuels during the next period, provided a license to handle these materials can be obtained. However, calculations and analysis on a curium-fueled thermoelectric generator of 100 watts electric output were performed.

One of the first requirements of this subtask was to supply sufficient data on fuel forms for the conceptual design of this thermoelectric generator for Task 5.7 .

Preliminary evaluations of the fuel and matrix material for this generator indicated that the curium metal with a nickel matrix is compatible. The size of the heat source at the time of encapsulation was calculated to be approximately 7450 thermal watts, 62 grams of curium metal and 205 kilocuries. The thermal analysis of this fuel with nickel matrix permitted a $20 / 1$ dilution ratio of nickel to curium without exceeding the melting point of the fuel pellets. Encapsulation of these pellets with suitable container materials is being evaluated further. Specifically, one of the major drawbacks of the nickel matrix material is its low thermal conductivity (see Table 17). Where radiation is the sole method of heat transfer, the nickel surface must be treated or coated with a material which will increase the emissivity to a value as close to 1.0 as possible.

The following equations were used to establish the fuel requirements of the 100-watt (electrical) generator (Task 5.7):

$$
P_{0}=\frac{P_{e}}{\eta_{u}}
$$

where

$$
\begin{aligned}
& P_{0}=\text { initial thermal input, watts } \\
& P_{e}=\text { initial electrical output, watts } \\
& \eta_{u}=\text { overall thermoelectric efficiency of generator. } \\
& M=\frac{P_{0}}{\rho_{\mathrm{Cm}}}
\end{aligned}
$$


where

$$
\begin{aligned}
{ }^{\rho} \mathrm{Cm} & =\text { specific power in thermal watts per gram } \\
M_{\mathrm{Cm}} & =\text { grams of curium } \\
\mathrm{V}_{\mathrm{Cm}} & =\text { volume of curium metal. }
\end{aligned}
$$

For calculating the electrical power output at various instants for a particular thermal input power, the following equation applies:

where

$$
P_{i}=\eta_{u} P_{o} e^{-\lambda t}
$$

$\begin{aligned} \eta_{u}= & \text { thermoelectrical conversion efficiency of generator, } \\ & \% \times 10^{-2}\end{aligned}$

$\lambda=$ decay constant of curium $=4.25 \times 10^{-3}$ days $^{-1}$

$t=$ time in days

$P_{i}=$ electrical power output at any instant.

From these equations, the following power requirements are calculated for the 100-watt generator with curium fuel.

\begin{tabular}{lccc} 
& $\begin{array}{c}\text { Decay Time } \\
\text { (days) }\end{array}$ & & $\begin{array}{c}\text { Thermal Power } \\
\text { (watts) }\end{array}$ \\
\cline { 2 - 2 } Out of reactor & 0 & 8850 \\
Encapsulation & 40 & & 7460 \\
Start of mission & 60 & & 5800 \\
End of miscian & 282 & & 9670
\end{tabular}

The fuel requirement out of the reactor in the form of curium, assuming a $100 \%$ recovery, is 74 grams of Curium -242 . For conversion of the americium to curium, approximately 172 grams of Americium241 must be irradiated in the reactor, assuming a $43 \%$ curium conversion. The curium when purified to eliminate all of the fission products and one-half of the Plutonium-238 was calculated to give an apparent density of the fuel and fuel matrix of $9.37 \mathrm{gm} / \mathrm{cc}$ at room temperature. To achieve the exact density, this apparent density must be corrected for the operating temperature of the capsule. 


\section{SUBTASK 6.4--HAZARDS ANALYSIS AND SHIELDING}

An investigation of the hazards of encapsulated fuel was initiated. Information on helium pressure buildup, capsule temperature and maximum allowable pressure for various containment materials is being compiled for evaluation of the hazards associated with each phase of the fuel history. This data can then be condensed into graphs and nomographs for future evaluations of the fuel forms without duplication of effort. Both nomographs and graphs will also be evaluated for applicability to the SNAP-type power units.

An example of the type of data which can be analyzed is shown by the equation used to represent the internal pressure buildup from helium generation within the fuel capsules.

$P=\frac{M_{H e} N_{0} R 1-e^{-\lambda t}\left(T_{0}-T_{a}\right) e^{-\lambda \cdot t}+T_{a}}{V M}$

where

$$
\begin{aligned}
& \mathrm{P} \quad=\text { pressure, atmosphere } \\
& \mathrm{M}_{\mathrm{He}}=\text { mass of a single helium atom }=6.645 \times 10^{-24} \text { grams } \\
& \mathrm{N}_{\mathrm{O}} \quad=\text { number of original radioisotope atoms in the source } \\
& \lambda \quad=\text { decay constant of the radioisotope, } \mathrm{sec}^{-1} \\
& \mathrm{t} \quad=\text { time, sec } \\
& \mathrm{T}_{\mathrm{a}} \quad=\text { ambient temperature, }{ }^{\circ} \mathrm{K} \\
& \mathrm{T}_{\mathrm{O}} \quad=\text { initial temperature of capsule, }{ }^{\circ} \mathrm{K} \\
& \mathrm{R} \quad=\text { constant }=0.08207 \text { liter--atmosphere } / \mathrm{mole} /{ }^{\circ} \mathrm{K} \\
& \mathrm{V} \quad=\text { volume of container, liter } \\
& \mathrm{M} \quad=\text { molecular weight of gas generated }
\end{aligned}
$$

NOTE: For a specific radioisotope, the internal pressure is a function of five variables:

$$
P=f\left(N_{o}, t, T_{o}, T_{a}, V\right)
$$




\section{Physical Properties of Curium}

Metallic Curium-242 is a silvery white metal similar in malleability to Polonium-210. It has been determined that it has a density of $13.5 \mathrm{gm} / \mathrm{cc}$ and a melting point of $950^{\circ} \mathrm{C}+50^{\circ}$. Up to this time, the density of curium had been reported to be about $7 \mathrm{gm} / \mathrm{cc}$, and the melting point was not known.

\section{Radiobiological Properties}

Curium-242 presents an internal hazard primarily. It is deposited in bone material, probably in the blood channels. With soluble compounds of the isotope, the critical organs are the lower large intestines and liver. In the insoluble forms, the critical organs are the lungs and the lower large intestines (Refs. 23 and 27).

\section{Shielding}

The shielding requirements for irradiated americium-aluminum slugs which have been exposed to a flux of $4 \times 10^{14}$ neutrons $/ \mathrm{sq} \mathrm{cm} /$ sec for a period of approximately 90 days are being determined to establish the design criteria for the shipping casks. The americium after irradiation converts to the constituents shown in Table 20.

TABLE 20

Americium Constituents*

$42 \%$ Curium -242

$29 \%$ Americium-241 (unconverted)

$9.2 \%$ Plutonium -242

8.0\% Plutonium -238

$1.7 \%$ Plutonium-240

$1.5 \%$ Plutonium -239

$0.7 \%$ Curium -243

$0.55 \%$ Americium-242 (100 year half-1ife)

\section{Type of Radiation}

$\alpha, \gamma$

$\alpha, \gamma, \mathrm{SF}$

$\alpha,--, \mathrm{SF}$

$\alpha, \gamma, \mathrm{SF}$

$\alpha, \gamma$

$\alpha, \gamma, \mathrm{SF}$

$\alpha, \gamma$

$\alpha, \beta, \gamma, \mathrm{K}$ 
TABLE 20 (continued)

Type of Radiation

$0.24 \%$ Cerium -244

$\alpha, \gamma$

$0.17 \%$ Americium -243

$\alpha, \gamma$

$0.01 \%$ Plutonium -241

$\alpha, \beta$

$6.9 \%$ Fission products (unidentified)

KEY: $\mathrm{SF}=$ Spontaneous Fission

$\alpha=$ Alpha

$B=$ Beta

$\gamma=$ Gamma

$\mathrm{K}=$ Capture

*Ref。 27

The shielding of the aluminum slugs must be designed to meet the government shipping requirements. The radiation from gammas, betas, alphas and neutrons emitted or formed in the material and the cask must be fully evaluated. Some spontaneous fission also results which may appreciably increase the heat generation. Since the aluminum slug melts at about $1220^{\circ} \mathrm{F}\left(660^{\circ} \mathrm{C}\right)$, provisions must be made in the cask to dissipate the internal heat and maintain the capsules well below this temperature。

For the irradiated 3.4 grams of americium in the aluminum slug, the radiation level 30 days after removal from the reactor should be of the order of 160 to 180 roentgen/hr at 4 feet from the source in air. The individual slugs will have to be shielded for a gamma radiation and a neutron radiation of about $1.8 \times 10^{8}$ neutrons per second for the protection of personnel. Preliminary calculations on 28 slugs indicate about 10 to 12 inches of lead is required plus a 12 - to 14 -inch neutron shield. Special means of cooling, designed to prevent any temperature excursion, are required to prevent exceeding the melting point of the lead or the fuel matrix. 
Blank page 


\section{XIII。SUBTASK 6.5--RADIATION DAMAGE TESTING}

This subtask includes the following:
A. Sample Preparation
B. Irradiation Testing
C. Evaluation

No effort was scheduled during this reporting period. 
Blank page 


\section{REFERENCES}

1. Goldman, D. $F_{\circ}$ and Singer, $S . F_{\circ}$ " Studies of a Minimum Orbital Unmanned Satellite of the Earth (Mouse) Part III Radiation Equilibrium and Temperatures."

2. Gredt, W.H., "Principles of Engineering Heat Transfer."

3. Spamer, A. M. "Information Pertaining to the Use of Curium-242 for Producing Heat," MND-1434.

4. Weddell, J。B., and Crane, W.W. T., Fersonal Communication.

5. Smith, A., Fields, P., Friedman, A., Cox, S, Sjoblom, R, "An Experimental Study of Fission in the Actinide Elements," Second U. N. Conference, Paper P690。

6. ANL5800, "Reactor Physics Constants."

7. Blizzard, E. P., "Nuclear Reactor Shielding," (A paper prepared for McGraw-Hill Book Company's Nuclear Reactor Handbook and reproduced for use at Oak Ridge School of Reactor Technology).

8. NBS Handbook 63, "Protection Against Neutron Radiation to 30 Million Electron Volts."

9. Strominger, D., Hollander, J.M. and Seaborg, G. T., "Table of Isotopes," Rev Mod Physics 30, No.2, Part II, April 1958.

10. Rockwell, J。, "Reactor Shielding Design Manual," U. S. Government Printing Office.

11. Goldstein, $H_{\circ}$, "The Attenuation of Gamma Rays and Neutrons in Reactor Shields," U. S. Government Printing Office.

12. Program Plan for Task 6, Advanced Fuel Technology Program (Contract AT-(30-3)-217, MND-P-2176 Rev 1, January 6, 1960.

13. Crane, W.W.T., "Preliminary Hazards Summary Report for the Martin Nuclear Laboratory," MND-2191-I, November 1959.

14. Crane, W.W.T., "Space Power," Institute Astronautical Federation, August and September 1959.

15. Crane, W.W.T., "Nuclear Energy," Power Source Conferences, April 1959. 
REFERENCES (continued)

16. King, A. M., "Evaluation of High Melting Cerium Compounds."

17. Moyer, H.V., "Polonium, tid-5221 AEC, Technical Information Service Extension, July 1956.

18. Seaborg, G. T., "Transuranic Elements," Nuclear Engineering Series。

19. "Properties of the Rare Earths," Rare Earth Research Council.

20. "Data on Chemicals for Ceramic Use," National Research Council No。118, June 1949 .

21. Second Geneva Conference on the Peaceful Uses of Atomic Energy, Papers on, Vol。6.

22. Seidell, $A_{\circ,}$ "Solubilities of Inorganic and Metal Organic Compounds," 4th Edition, Vol。1.

23. Crane, W. W. To, "Preliminary Hazards Summary Report for The Martin Nuclear Laboratory," MND-1831A, August 1959.

24. Reviews of Modern Physics, Vol. 30, No.2, Part II, April 1958.

25. The Iron Age, p。155, November 12, 1959.

26. Cilimberg, $R_{\circ} I_{\circ}$ "Brief Summary of Materials for the Containment of Radioisotopes for Reentry or Burnup," MND-P-2162, October 26,1959 。

27. "Americium-241 and Curium-242 Fuel Processing Hazards," MND-P-1972, August 1959。

28. The Reactor Handbook, Vol. 3 Sec.1--General Properties of Materials, AECD 3647 March 1955.

29. Materials Handbook--1948 Edition.

30. WADC $-\mathrm{TR}-56-400$ 。

31. Materials Engineering Bulletin 112-59.

32. DMIC Memorandum 19. 


\section{REFERENCES (continued)}

33. Materials Selector 1958 to 1959 Reference Issue.

34. "Radioisotope Fueled Auxiliary Power Unit," Quarterly Progress Report, MND-P-3007 April to June 1959, SECRET RESTRICTED DATA

35. Higgens, $\mathrm{G}_{\circ} \mathrm{H}_{\circ}$, and Crane, W. W. T., "The Production and Chemical Isolation of Curium-242 in Thousand-Curie Quantities, "UCRL 5021, University of California Radiation Laboratory, Livermore Site, California, March 31, 1958.

36. Magnusson, L。 B., and Anderson, M. L., Journal of Americium Chemical Society 76, 6207, 1954 .

37. Crane, W.W.T., and Higgens, G. H., "Aluminum Decladding Procedure by Volatilization of Aluminum Chloride," UCRL 4357, University of California Radiation Laboratory, Livermore Site, California, July 13, 1954. 


\section{APPENDIX A}

\section{HELIUM PRESSURE CALCULATIONS}

$$
\mathrm{P}=\frac{\mathrm{mRT}}{\mathrm{VM}}
$$

where

$$
\begin{aligned}
& \mathrm{P}=\text { pressure, atmospheres } \\
& \mathrm{m}=\text { mass of helium gas generated, grams } \\
& \mathrm{V}=\text { volume of gas, liters } \\
& \mathrm{T}=\text { temperature, }{ }^{\circ} \mathrm{K} \\
& \mathrm{M}=\text { molecular weight of gas generated }(\mathrm{He}=4.003) \\
& \mathrm{R}=\text { universal gas constant, } 0.08207 \frac{\text { liter-atmosphere }}{\text { mole }-{ }^{\circ} \mathrm{K}} \\
& \mathrm{m}=\mathrm{m}_{\mathrm{He}} \mathrm{N}_{\mathrm{O}}\left(1-\mathrm{e}^{-\lambda^{\mathrm{t}}}\right)
\end{aligned}
$$

where

$$
\begin{aligned}
\mathrm{m}_{\mathrm{He}} & =\text { mass of He atom }=6.6456 \times 10^{-24} \mathrm{gm} \\
\mathrm{N}_{\mathrm{O}} & =\text { Number of original atoms of fuel } \\
\lambda & =\text { fuel decay constant }=2.54 \times 10^{-10} \mathrm{sec}^{-1} \\
\mathrm{t} & =\text { time, seconds } \\
\mathrm{T} & =\left(\mathrm{T}_{\mathrm{O}}-\mathrm{T}_{\mathrm{A}}\right) \mathrm{e}^{-\lambda t}+\mathrm{T}_{\mathrm{A}}
\end{aligned}
$$

where

$$
\begin{aligned}
& \mathrm{T}_{\circ}=\text { initial temperature }{ }^{\circ} \mathrm{K}=866.3^{\circ} \mathrm{K} \\
& \mathrm{T}_{\mathrm{A}}=\text { ambient temperature }{ }^{\circ} \mathrm{K}=200^{\circ} \mathrm{K} .
\end{aligned}
$$

Substituting Eqs.(2) and (3) into Eq. (1), 


$$
P=\frac{R m_{H e} N_{0}\left(1-e^{-\lambda t}\right)\left(T_{0}-T_{A}\right) e^{-\lambda t}+T_{A}}{V M}
$$

Taking the derivative of $P$ with respect to $t$ and setting it equal to zero, we obtain

$$
\frac{d P}{d t}=\frac{m_{H e} e^{R N}}{V M} \quad\left[2 \lambda e^{-2 \lambda t}\left(T_{0}-T_{A}\right)+\lambda e^{-\lambda t}\left(2 T_{A}-T_{0}\right)\right]=0 .
$$

Substituting known values and solving for $t$, we obtain

$t=383$ years (time of maximum pressure).

Also, the amount of fuel required is 54.4 grams of $\mathrm{Pu}-238$.

Therefore,

$N_{0}=\frac{\text { grams of fuel } \times \text { Avogadros number }}{\text { molecular weight }}$.

$\frac{54.4}{238} \times 6.02 \times 10^{23}=1.376 \times 10^{23}$ atoms.

Substituting the above values of $t$ and $N_{0}$ in Eq. (4) and solving for $P$ in terms of $V$, we obtain

$$
\begin{aligned}
& P= \frac{0.08207 \times 6.6456 \times 10^{-24} \times 1.376 \times 10^{23}}{4.003 \mathrm{~V}}\left[1-\mathrm{e}^{\left(2.2 \times 10^{-5}\right)}\right. \\
&\left.\cdot\left(1.399 \times 10^{-5}\right)\right]\left[(866-200) \mathrm{e}^{\left(-2.2 \times 10^{-5}\right)\left(1.399 \times 10^{-5}\right)}+200\right] \\
& P=\frac{4.18}{V}
\end{aligned}
$$

where

$$
\begin{aligned}
& \mathrm{V}=\text { liters } \\
& \mathrm{P}=\text { atmospheres. }
\end{aligned}
$$


APPENDIX B

THERMAL ANALYSIS

1-WATT ELECTRIC GENERATOR SYSTEM FOR SPACE APPLICATION

\section{A. DESIGN REQUIREMENTS}

The system is to consist of a Plutonium-238 fueled electric power generator and a DC-to-DC converter. The required output of the power supply is 1 watt at 15 volts. The generator must have an output of 1.5 watts at 1.5 volts.

The equivalent circuit is shown by the following sketch:

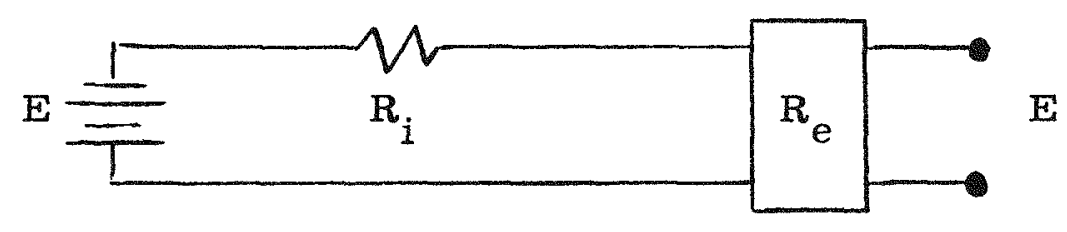

\section{B. THERMOELECTRIC ELEMENTS}

The thermoelectric elements considered are the cast lead telluride type manufactured by Minnesota Mining and Manufacturing Company. The $\mathrm{P}$ elements are doped with $1.0 \%$ sodium and the $\mathrm{N}$ elements are doped with $0.03 \%$ lead iodide. The characteristics of these elements operating at temperatures between $538^{\circ} \mathrm{C}\left(1000^{\circ} \mathrm{F}\right)$ and $149^{\circ} \mathrm{C}\left(300^{\circ} \mathrm{F}\right)$ are listed below (integrated average values).

\section{Electrical Resistivity}

$$
\begin{aligned}
& \rho_{\mathrm{n}}=3.08 \times 10^{-3} \mathrm{ohm}-\mathrm{cm} \\
& \rho_{\mathrm{p}}=3.71 \times 10^{-3} \mathrm{ohm}-\mathrm{cm}
\end{aligned}
$$

2. Thermal Conductivity

$$
K_{n}=1.63 \times 10^{-2} \text { watts } / \mathrm{cm}^{\circ} \mathrm{C}
$$




$$
\mathrm{K}_{\mathrm{p}}=1.94 \times 10^{2} \text { watts } / \mathrm{cm}^{\circ} \mathrm{C}
$$

\section{Seebeck Coefficient}

$$
\begin{aligned}
e & =\frac{/ e_{\mathrm{p}} /+/ e_{\mathrm{n}} /}{\Delta \mathrm{T}}=\frac{0.0937+0.0950}{389} \\
& =4.85 \times 10^{-4} \text { volts } /{ }^{\circ} \mathrm{C}
\end{aligned}
$$

\section{DETERMINATION OF ELEMENT SIZES}

An algebraic summation of the voltages in the primary part of the equivalent circuit gives the equation

$$
E=E_{i}+E_{E}=I\left(R_{i}+R_{e}\right)
$$

$\mathrm{E}$ is the total emf of the thermoelectric elements, or $\mathrm{Ne} \Delta \mathrm{T}$, where

$$
\begin{aligned}
& N=\text { number of couples used } \\
& e=\text { Seebeck coefficient of each couple } \\
& \Delta T=\text { Hot junction minus cold junction temperatures } \\
& I=E_{e}=\text { current in primary circuit } \\
& E_{i}=\text { voltage drop across the elements } \\
& E_{e}=\text { voltage drop across load } \\
& R_{i}=\text { internal resistance of the elements } \\
& D_{e}=\text { iuau insueciance. }
\end{aligned}
$$

Therefore,

$$
\begin{aligned}
N e_{\Delta T} & =\frac{E_{e}\left(R_{i}+R_{e}\right)}{R_{e}} \\
& =\frac{E_{e}\left(1+R_{e} / R_{i}\right)}{R_{e} / R_{i}} .
\end{aligned}
$$


For maximum generator efficiency,

$$
\frac{\mathrm{R}_{\mathrm{e}}}{\mathrm{R}_{\mathrm{i}}}=\left[1+\left(\frac{\mathrm{T}_{\mathrm{h}}+\mathrm{T}_{\mathrm{c}}}{2}\right)\left(\frac{e}{\sqrt{\mathrm{k}_{\mathrm{p}} \rho_{\mathrm{p}}}+\sqrt{\mathrm{k}_{\mathrm{n}} \rho_{\mathrm{n}}}}\right)^{2}\right]^{1 / 2}=1.27 .
$$

NOTE: This relationship was derived from an expression for Carnot efficiency.

$$
N=\frac{E_{e}}{\ell \Delta T}\left(\frac{2.27}{1.27}\right)=9.47 \mathrm{E}_{\mathrm{e}^{\circ}}
$$

An expression relating length-to-area ratio of elements and generator output requirements can be derived as follows:

$\mathrm{R}_{\mathrm{i}} /$ couple $=\frac{\mathrm{p}_{\ell_{\mathrm{p}}}}{\mathrm{A}_{\mathrm{p}}}+\frac{\mathrm{n}_{\ell_{\mathrm{n}}}}{\mathrm{A}_{\mathrm{n}}}$

$\ell=$ length of the elements

$A=$ cross-sectional area of elements.

For practical purposes, let $\ell_{\mathrm{p}}=\ell_{\mathrm{n}}$ and $A_{\mathrm{p}}=\mathrm{A}_{\mathrm{n}}$; then, $\mathrm{R}_{\mathrm{i}} /$ couple $=$ $\left(\rho_{\mathrm{p}}+\rho_{\mathrm{n}}\right) \ell / \mathrm{A}=6.79 \times 10^{-3}\left(\frac{\ell}{\mathrm{A}}\right)$

$$
\begin{aligned}
R_{\dot{i}} & =6.79 \times 10^{-3}\left(\frac{\ell}{A}\right) N \\
& =6.79 \times 10^{-3}\left(\frac{\ell}{A}\right)\left(9.47 E_{e}\right)=\frac{R_{e}}{1.27} \\
R_{e} & =\frac{E_{e}^{2}}{W} \\
W & =\text { output power of generator } \\
\frac{\ell}{A} & =12.24 \frac{E_{e}}{W} \\
\frac{\ell}{A} & =1 / 0.786 D^{2} \\
D & =\text { diameter of elements. }
\end{aligned}
$$


Figure 70 is a plot of $\ell / A$ versus $E_{e}$ at various values of $W$ and $\ell / A$ versus $\ell$ at various values of $D$. From this graph, element sizes can readily be found from desired generator output. For the specific generator under consideration, $1 / 4$ inch diameter by $1-1 / 2$ inch long elements were selected. An output of 1.5 volts requires $9.45(1.5)$ or 15 couples.

\section{ELEMENT ARRANGEMENT}

The selection of an arrangement for the thermoelectric elements was based on the following limitations:

(1) Size and position of the heat dissipator (radiator) as dictated by the space satellite application of the generator system.

(2) Configuration of the packaged heat source as dictated by required radiation shielding, re-entry requirements and available form of radioisotope.

(3) Manufacture of detailed parts.

(4) Field assembly of the generator.

(5) Thermal efficiency.

(6) Weight.

One of the design requirements of this system is that the radiator be an integral part of the satellite skin. The design concept shown in Fig. B-1 satisfies this requirement.

Practical element patterns are 2 rows at 15 elements each, 3 rows of 10 elements each and 5 rows of 6 elements each. Two rows of 15 elements each were selected as the pattern which would provide for the most efficient radiator area.

\section{E. HEAT FLOW CALCULATIONS}

A calculation of the heat losses from the selected configuration of the generator as shown in Fig. B-1 will give the size of heat source required. This calculation will also give the thermal efficiency of the generator. To simplify the calculations, it is assumed that the re are no edge effects (parallel heat flow). Further assumptions are: 

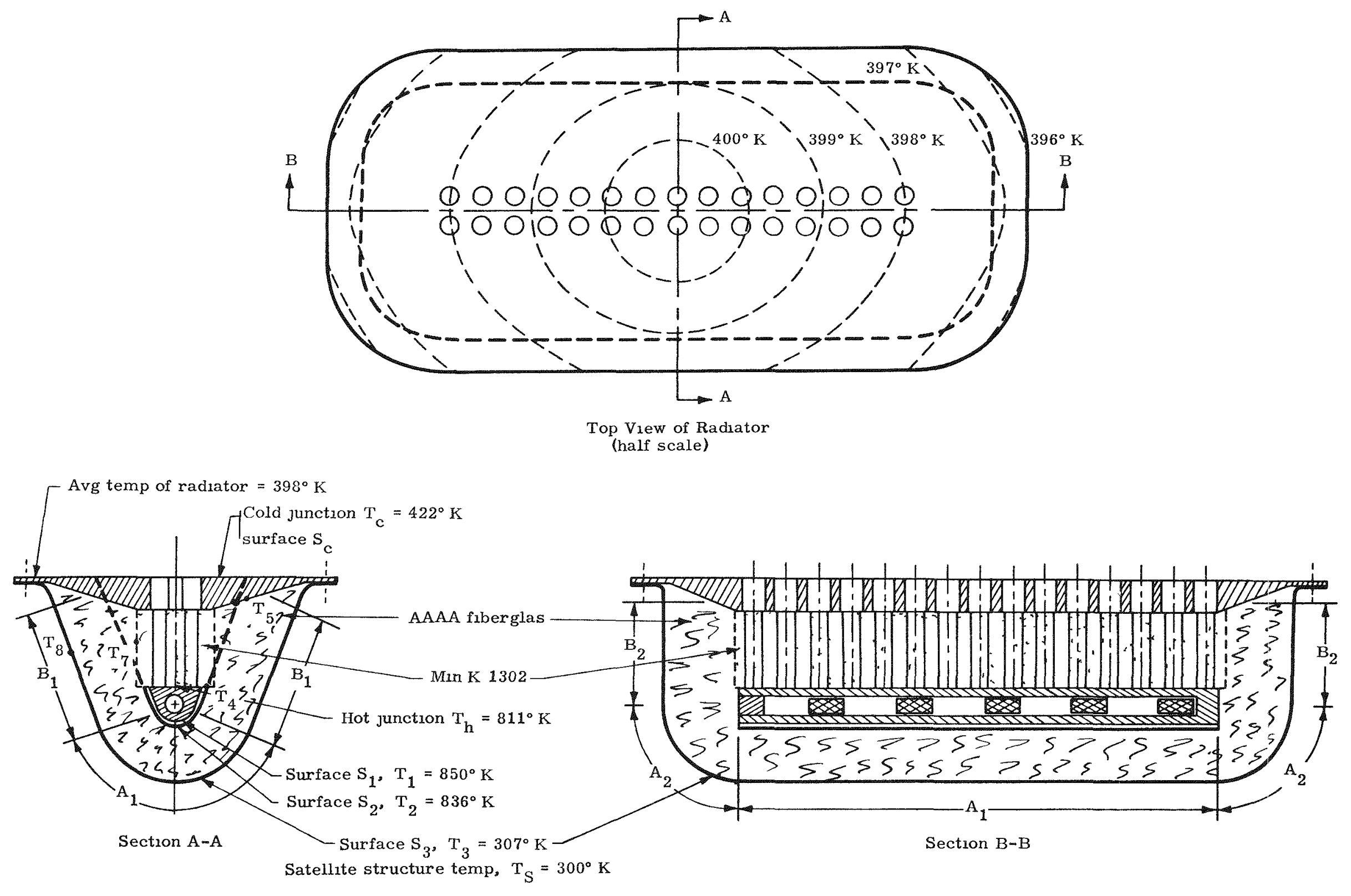
(1) Internal satellite temperature $=300^{\circ} \mathrm{K}\left(27^{\circ} \mathrm{C}\right)$.

(2) Satellite internal structure radiated to by the surface of the generator is $2-1 / 2$ times the generator surface.

(3) The hot junction temperature $=811^{\circ} \mathrm{K}\left(538^{\circ} \mathrm{C}\right)$.

(4) Temperature drop across heat source envelope $=39^{\circ} \mathrm{C}$.

(5) Cold junction temperature $=422^{\circ} \mathrm{K}\left(149^{\circ} \mathrm{C}\right)$.

The generator was divided into segments for heat flow consideration. 1. Flow Through Elements

The heat flow through the 30 thermoelectric elements due to $\Delta \mathrm{T}$ (assuming no side losses) is

$$
\begin{aligned}
q_{A} & =\frac{N\left(k_{p}+k_{n}\right)}{\ell / A} \Delta T \\
& =\frac{15\left(3.57 \times 10^{-2}\right)}{12} \text { (389) } \\
& =17.36 \text { watts. }
\end{aligned}
$$

\section{Flow Through Min-K Insulation}

The heat flow through the Min-K insulation surrounding the 30 thermoelectric elements is

$$
\begin{aligned}
\mathrm{q}_{\mathrm{B}} & =\frac{\mathrm{k}_{(\mathrm{Min}-\mathrm{K})} \mathrm{A}\left(\mathrm{T}_{\mathrm{h}}-\mathrm{T}_{\mathrm{c}}\right)}{\ell} \\
\mathrm{A} & =24(4)-30(0.786)(0.3125)^{2}(6.45) \\
& =81.15 \mathrm{~cm}^{2} \\
\ell & =3.81 \mathrm{~cm} \\
\mathrm{~T}_{\mathrm{h}}-\mathrm{T}_{\mathrm{C}} & =389^{\circ} \mathrm{K} \\
\mathrm{q}_{\mathrm{B}} & =\frac{3.3 \times 10^{-4}(81.15)(389)}{3.81} \\
& =2.734 \text { watts. }
\end{aligned}
$$




\section{Flow Through Structure}

The structure that holds the heat source in place is a cradle with four legs which are attached to the under side of the radiator (see Fig. B-1). These legs are stainless steel $1.27 \mathrm{~cm}$ wide and $0.076 \mathrm{~cm}$ thick.

$$
\begin{aligned}
& { }_{\mathrm{C}}=\frac{\mathrm{kA}\left(\mathrm{T}_{4}-\mathrm{T}_{5}\right)}{\ell} \\
& \mathrm{k}=0.265 \text { watts } / \mathrm{cm}-{ }^{\circ} \mathrm{C} \\
& \mathrm{A}=(0.0965)(4)=0.386 \mathrm{~cm}^{2} \\
& \ell=5 \mathrm{~cm} \\
& \mathrm{~T}_{4}-\mathrm{T}_{5}=810-410=400^{\circ} \mathrm{K} \\
& { }_{\mathrm{C}}=8.19 \text { watts. }
\end{aligned}
$$

4. Flow Through Section $A_{1}$

The heat flow through the section under the heat source (see Fig. B-1) is by radiation from $S_{1}$ (bottom surface of heat source) to $S_{2}$ (bottom surface of heat source support), by conduction through the AAAA insulation to $\mathrm{S}_{3}$ (bottom of outer skin of generator) and by radiation from $S_{3}$ to the satellite structure.

Radiation from $S_{1}$ to $S_{2}$

$$
\begin{aligned}
\mathrm{q}_{\mathrm{D}} & =\frac{\sigma \mathrm{A}_{1}}{1 / \epsilon_{1}+\mathrm{A}_{1} / \mathrm{A}_{2}\left(1 / \epsilon_{2}-1\right)}\left(\mathrm{T}_{1}^{4}-\mathrm{T}_{2}^{4}\right) \\
\sigma & =5.67 \times 10^{-12} \text { watts } / \mathrm{cm}^{2}-{ }^{\circ} \mathrm{K}^{4} \\
\mathrm{~A}_{1} & =2 \pi \mathrm{r}_{1}\left(\frac{142}{360}\right) \ell=2 \pi(0.8)\left(\frac{142}{360}\right)(22.86) \\
& =45.4 \mathrm{~cm}^{2} \\
\mathrm{~A}_{2} & =45.4\left(\frac{1}{0.8}\right)=56.8 \mathrm{~cm}^{2} \\
\epsilon_{1} & =0.8(\text { Hastelloy) }
\end{aligned}
$$


B-8

$$
\begin{aligned}
& \epsilon_{2}=0.03 \text { (gold surface) } \\
& \mathrm{q}_{\mathrm{D}}=9.51 \times 10^{-12}\left(\mathrm{~T}_{1}^{4}-\mathrm{T}_{2}^{4}\right) .
\end{aligned}
$$

Conduction from $\mathrm{S}_{2}$ to $\mathrm{S}_{3}$

$$
\begin{aligned}
\mathrm{q}_{\mathrm{D}} & =-\mathrm{K}_{(\text {AAAA })} \mathrm{A} \frac{\mathrm{dT}}{\mathrm{dr}} \\
\mathrm{A} & =2 \pi \mathrm{r}\left(\frac{142}{360}\right) \ell=2 \pi\left(\frac{142}{360}\right)(23) \mathrm{r} \\
& =56.97 \mathrm{r} \mathrm{cm}{ }^{2} \\
\mathrm{q}_{\mathrm{D}} & =-\mathrm{K}_{(\text {AAAA })} 56.97 \frac{\mathrm{dT}}{\mathrm{dr}} \\
& =\frac{56.97}{\ell \mathrm{n}_{3} / \mathrm{r}_{2}}\left(\mathrm{~T}_{2}-\mathrm{T}_{3}\right) \\
& =1.44 \times 10^{-5} \text { watts } / \mathrm{cm}-{ }^{\circ} \mathrm{C} \\
\mathrm{K} & \ell \mathrm{n} \mathrm{r}_{3} / \mathrm{r}_{2}=\ell \frac{3.8}{1}=1.33 \\
\mathrm{q}_{\mathrm{D}} & =52.6 \times 10^{-5}\left(\mathrm{~T}_{2}-\mathrm{T}_{3}\right)
\end{aligned}
$$

Radiation from $S_{3}$ to $S_{S}, S_{S}$ surface of satellite structure.

$$
\begin{aligned}
\mathrm{q}_{\mathrm{D}} & =\frac{\sigma \mathrm{A}_{3}}{1 / \epsilon_{3}+\mathrm{A}_{3} / \mathrm{A}_{\mathrm{S}}\left(1 / \epsilon_{\mathrm{S}}-1\right)}\left(\mathrm{T}_{3}^{4}-\mathrm{T}_{\mathrm{S}}^{4}\right) \\
\mathrm{A}_{3} & =2 \pi \mathrm{r}_{3}\left(\frac{142}{360}\right) \ell=2 \pi(3.8)\left(\frac{142}{360}\right)(23) \\
& =216 \mathrm{~cm}^{2} \\
\mathrm{~A}_{\mathrm{S}} & =21 / 2 \mathrm{~A}_{3}=541 \\
\epsilon_{3} & =0.6 \text { (stainless steel) }
\end{aligned}
$$




$$
\begin{aligned}
\epsilon_{\mathrm{s}} & =0.2 \text { (aluminum) } \\
\mathrm{q}_{\mathrm{D}} & =3.74 \times 10^{-10}\left(\mathrm{~T}_{3}^{4}-\mathrm{T}_{\mathrm{s}}^{4}\right)
\end{aligned}
$$

Equations $(4),(5)$ and $(6)$ can be solved simultaneously for $q_{D}$ giving

$$
\begin{aligned}
& \mathrm{q}_{\mathrm{D}}=0.3 \text { watt } \\
& \mathrm{T}_{2}=836^{\circ} \mathrm{K} \\
& \mathrm{T}_{3}=307^{\circ} \mathrm{K}
\end{aligned}
$$

5. Flow Through Section B, (Fig. B-1)

$$
\begin{aligned}
\mathrm{q}_{\mathrm{E}} & =\mathrm{KA} \frac{\left(\mathrm{T}_{7}-\mathrm{T}_{8}\right)}{\ell} \\
\mathrm{K} & =1.44 \times 10^{-5} \text { watts } / \mathrm{cm}-{ }^{\circ} \mathrm{C} \\
\mathrm{A} & =2(24)(6.6)=316.8 \mathrm{~cm}^{2} \\
\ell & =3.5 \mathrm{~cm} \\
\mathrm{~T}_{7}-\mathrm{T}_{8}=600-352=248^{\circ} \mathrm{C} & =0.323 \text { watts }
\end{aligned}
$$

6. Flow Through Section $\mathrm{A}_{2}$ (Fig. B-1)

Conduction through insulation

$$
\begin{aligned}
& \mathrm{q}_{F}=-\mathrm{KA} \frac{\mathrm{dt}}{\mathrm{dr}} \\
& \mathrm{A}=4 \pi \mathrm{r}^{2}\left(\frac{172}{360}\right)=6.01 \mathrm{r}^{2} \mathrm{~cm}^{2} \\
& \mathrm{~K}=1.44 \times 10^{-5} \text { watts } / \mathrm{cm}-{ }^{\circ} \mathrm{C} \\
& \mathrm{q}_{\mathrm{F}}=\frac{8.65 \times 10^{-5}}{1 / \mathrm{r}_{1}-1 / \mathrm{r}_{3}}\left(\mathrm{~T}_{1}-\mathrm{T}_{3}\right) \\
& \mathrm{r}_{1}=0.8 \mathrm{~cm} ; \mathrm{r}_{3}=4.3 \mathrm{~cm}
\end{aligned}
$$




$$
\begin{aligned}
& \mathrm{T}_{1}=850^{\circ} \mathrm{K} \\
& \mathrm{q}_{\mathrm{F}}=0.0496-5.85 \times 10^{-5} \mathrm{~T}_{3}
\end{aligned}
$$

Radiation from $\mathrm{S}_{3}$ to satellite structure $\mathrm{S}_{\mathrm{S}}$.

$$
\begin{aligned}
& \mathrm{q}_{\mathrm{F}}=\frac{\sigma \mathrm{A}_{2}}{1 \epsilon_{3}+\mathrm{A}_{2} / \mathrm{A}_{\mathrm{S}}\left(1 / \epsilon_{\mathrm{s}}-1\right)}\left(\mathrm{T}_{3}^{4}-\mathrm{T}_{\mathrm{s}}^{4}\right) \\
& \mathrm{A}_{2}=4 \pi \mathrm{r}_{3}^{2}\left(\frac{172}{360}\right)=1 / / \mathrm{cm}^{2} \\
& A_{S}=2.5 \mathrm{~A}_{2} \\
& \mathrm{~T}_{\mathrm{S}}=300^{\circ} \mathrm{K} \\
& \mathrm{q}_{\mathrm{F}}=1.93 \times 10^{-10}\left(\mathrm{~T}_{3}^{4}-300^{4}\right) \\
& \mathrm{q}_{\mathrm{F}}=1.93 \times 10^{-10} \mathrm{~T}_{3}^{4}-1.56
\end{aligned}
$$

Equations $(8)$ and $(9)$ can be solved simultaneously for $q_{F}$ and $T_{3}$ to give:

$$
\begin{aligned}
& \mathrm{q}_{\mathrm{F}}=0.038 \text { watts } \\
& \mathrm{T}_{3}=302^{\circ} \mathrm{K}
\end{aligned}
$$

7. Flow Through Section $\mathrm{B}_{2}$ (Fig. B-1)

Conduction through insulation.

$$
\begin{aligned}
{ }_{G}^{q_{G}} & =-K A \frac{d t}{d r} \\
K & =1.44 \times 10^{-5} \text { watts } / \mathrm{cm}^{\circ} \mathrm{C} \\
A & =2 \pi \mathrm{rl} \\
\mathrm{q}_{\mathrm{G}} & =2 \pi \mathrm{Kr}_{\ell} \frac{\mathrm{dt}}{\mathrm{dr}}=\frac{2 \pi \mathrm{K}_{\ell}}{\ell \mathrm{n}_{3} / \mathrm{r}_{1}}\left(\mathrm{~T}_{1}-\mathrm{T}_{3}\right) \\
\ell & =5 \mathrm{~cm} \\
\ell \mathrm{n} \mathrm{r}_{3} / \mathrm{r}_{1} & =\ell \mathrm{n} 7 / 1.5=1.55
\end{aligned}
$$




$$
\begin{aligned}
\mathrm{T}_{1} & =850^{\circ} \mathrm{K} \\
\mathrm{q}_{\mathrm{G}} & =0.248-2.92 \times 10^{-4} \mathrm{~T}_{3}
\end{aligned}
$$

Radiation from $S_{3}$ to $S_{s}$

$$
\begin{aligned}
\mathrm{q}_{G} & =\frac{\sigma A_{3}}{1 / \epsilon_{3}+A_{3} / A_{s}\left(1 / \epsilon_{s}-1\right)}\left(T_{3}^{4}-T_{s}^{4}\right) \\
\sigma & =5.67 \times 10^{-12} \text { watts } / \mathrm{cm}^{2}-0 \mathrm{~K}^{4} \\
A_{3} & =2 \pi r_{3} \ell=2 \pi 7(5) \\
& =220 \mathrm{~cm}^{2} \\
A_{S} & =2.5 A_{3} \\
T_{S} & =300^{\circ} \mathrm{K} \\
\epsilon_{3} & =0.6 \\
\epsilon_{s} & =0.2 \\
q_{G} & =3.815 \times 10^{-10} \mathrm{~T}_{3}^{4}-3.086
\end{aligned}
$$

Solve equations (10) and (11) simultaneously for $q_{G}$ and $T_{3}$ to obtain

$$
\begin{aligned}
{ }_{G} & =0.167 \text { watt } \\
T_{3} & =304^{\circ} \mathrm{K}
\end{aligned}
$$

8. Summary of Heat Losses

Through elements $\left(\mathrm{q}_{\mathrm{A}}\right)$

Through Min $\mathrm{K}\left(\mathrm{q}_{\mathrm{B}}\right)$

Through structure $\left(q_{C}\right)$

Other losses $\left(q_{D}+q_{E}+q_{F}+q_{G}\right.$

Total losses

\subsection{6 watts}

2.73

8.19

0.83 
B -12

9. Calculation of Efficiencies

$$
\begin{aligned}
& \text { Overall generator efficiency }=\frac{1.5(100)}{29.11+1.5}=4.9 \% \\
& \text { Overall system efficien } \approx y=\frac{1(100)}{29.11+1.5}=3.3 \% \\
& \text { Thermal efficiency } \\
& =\frac{17.36(100)}{29.11+1.5}=56.7 \%
\end{aligned}
$$




\section{APPENDIX C}

\section{THERMOELECTRIC GENERATOR OPTIMIZATION CODE}

This appendix describes the computer program used in the analysis of the radiative boundary spherical generators in Subtask 5.7. The main purpose of this design program is to outline the general range of parameters for any given design requirements. Since it is based on a spherical generator shape, the applicability of the calculated values to the actual design values will depend on how closely the design approaches a sphere.

\section{A. PROCEDURE}

The code is basically a repetitive 33-step operation, based on 21 equations and including one trial and error determination. Calculating by hand, each series of 33 steps takes about two hours. Using the program, approximately four minutes of machine time is required for a series of 56 calculations. The time per calculation decreases with increasing number of calculations.

The generator configuration used in the program consists of three concentric spheres. The smallest is the heat source, which radiates its heat to the second sphere, the hot junction. Between the outer sphere (cold junction) and hot junction are uniformly distributed thermoelectric elements surrounded by insulation. Temperature distributions are assumed to be uniform.

The series of 33 steps proceeds as follows:

The first nine steps are devoted to selecting the operating temperatures and the materials with their particular characteristics.

(1) Fuel material--This specifies the power density and specific gravity.

(2) Clad material--Characteristics needed here are density, emissivity, and temperature. The thermal characteristics of the clad material determine the maximum temperature of the inner sphere.

(3) Support material--The cross-sectional area and the density of the support material are required to find the total weight. It is assumed that the heat conducted through these supports is negligible.

(4) Hot junction material--Density, emissivity, and shell thickness are necessary. 
(5) Thermoelectric material--Density and hot junction temperature are determined by this selection.

(6) Cold junction material--Density, infrared emissivity, thickness, and absorptivity for solar radiation are determined.

(7) Insulation material--Density and thermal conductivity are the properties of interest.

(8) Power output.

(9) Cold junction temperatur.

The actual computation begins with step 10. The equations used are listed following the calculation procedure.

(10) $Z_{c}-$ composite figure of merit -eq. (1)

(11) $\mathrm{X}_{\mathrm{m}}$ - resistance ratio - eq. (2)

(12) $\eta_{\mathrm{el}}$ - thermoelectric efficiency- eq. (3)

(13) $\mathrm{q}_{\text {res }}$ - reversible energy rate (i.e., heat transmitted through the thermoelectrics due to the Peltier effect) eq. (4)

(14) $\mathrm{A}_{\text {ther }}$ - thermoelectric area - eq. (5)

(15) $\mathrm{q}_{\text {ther }}$ - heat conducted through thermoelectrics, eq. (6)

(16) Select $t^{\text {-thermal efficiency }(0.9)}$

(17) $\mathrm{A}_{3}-$-cold junction area--eq. (7)

(18) $A_{2}--$ hot junction a rea--eq. (8)

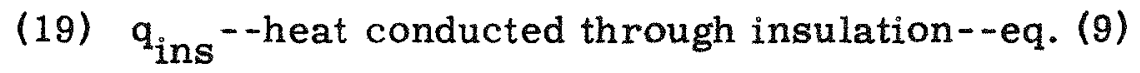

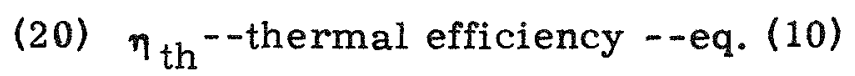

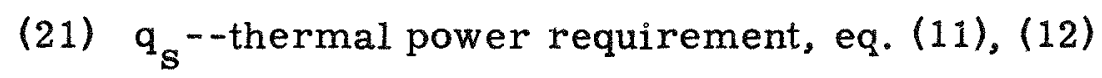

As a computation check, the results of the calculations by each should be approximately equal ( $<3 \%$ difference). 
(22) $A_{1}$--surface area of source cladding, eq. (13)

(23) $\mathrm{w}_{\mathrm{s}}{ }^{-- \text {source weight--eq. (14) }}$

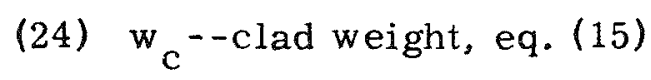

(25) $\mathrm{w}_{\text {sup }}{ }^{- \text {-support weight, eq. (16) }}$

(26) $w_{\text {i.s. }}$ - -weight of inner shell (hot junction), eq. (17)

(27) $\mathrm{w}_{\text {ther }}{ }^{--w e i g h t}$ of the thermoelectrics, eq. (8)

(28) $w_{\text {ther }} \times 0.25$ - weight of electrical connectors

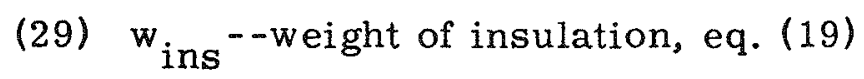

(30) wo.s.-- weight of outer shell (cold junction) eq. (20)

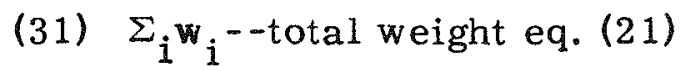

(32) Return to step 9.

(33) Repeat series until sufficient data are obtained for a plot of cold junction temperatures vs. weight for any thermoelectric length. 
C -4

B. EQUATIONS USED IN PROGRAMI

$z_{c}=\frac{\left[e_{N}+e_{P}\right]^{2}}{\left[\sqrt{k_{P} \rho_{P}}+\sqrt{k_{N} \rho_{N}}\right]^{2}}$

where

$\mathrm{e}=$ Seebeck coefficient $\left(\right.$ volts $\left./{ }^{\circ} \mathrm{C}\right)$

$\mathrm{k}=$ thermal conductivity of the thermoelectric (watts $/ \mathrm{cm}-{ }^{\circ} \mathrm{C}$ )

$\rho=$ electrical resistivity of the thermolectrics (ohm $-\mathrm{cm}$ )

subscripts $\mathrm{P}$ and $\mathrm{N}$ refer to $\mathrm{P}$ - and $\mathrm{N}$-type thermoelectrics, respectively.

$x_{m}=\sqrt{1+z_{c}\left(\frac{T_{2}+T_{3}}{2}\right)}$

where

$T_{2}=$ the hot junction temperature

$\mathrm{T}_{3}=$ the cold junction temperat ure.

$\eta_{\mathrm{el}}=\frac{\mathrm{x}_{\mathrm{m}}\left[\mathrm{T}_{2}-\mathrm{T}_{3}\right]}{\left(1+\mathrm{x}_{\mathrm{m}}\right) \mathrm{T}_{2}+\frac{\left(1+\mathrm{x}_{\mathrm{m}}\right)^{2}}{\mathrm{z}_{\mathrm{c}}}-\frac{\mathrm{T}_{2}-\mathrm{T}_{3}}{2}}$

$q_{\text {res }}=\frac{1+x_{m}}{x_{m}} w_{e} \frac{T_{2}}{T_{2}-T_{3}}$

where

$W_{e}=$ the output power of the generator 


$$
A_{\text {ther }}=\left[\frac{1+\sqrt{\frac{k_{N} \rho_{P}}{k_{P} \rho_{N}}}}{e\left(T_{2}-T_{3}\right) \frac{{ }_{m}}{1+x_{m}}}\right]
$$

$$
\left[\frac{\frac{\rho_{\mathrm{P}}^{\ell}}{\sqrt{\frac{\mathrm{k}_{\mathrm{N}} \rho_{\mathrm{P}}}{\mathrm{k}_{\mathrm{P}} \rho_{\mathrm{N}}}}}+\rho_{\mathrm{N}} \ell}{\frac{\mathrm{e}\left(\mathrm{T}_{2}-\mathrm{T}_{3}\right)}{\mathrm{W}_{\mathrm{e}}\left(1+\mathrm{x}_{\mathrm{m}}\right)}}\right]
$$

where

$$
\begin{aligned}
& e=e_{P}+e_{N} \\
& \ell=\text { the length of the thermoelectrics }
\end{aligned}
$$

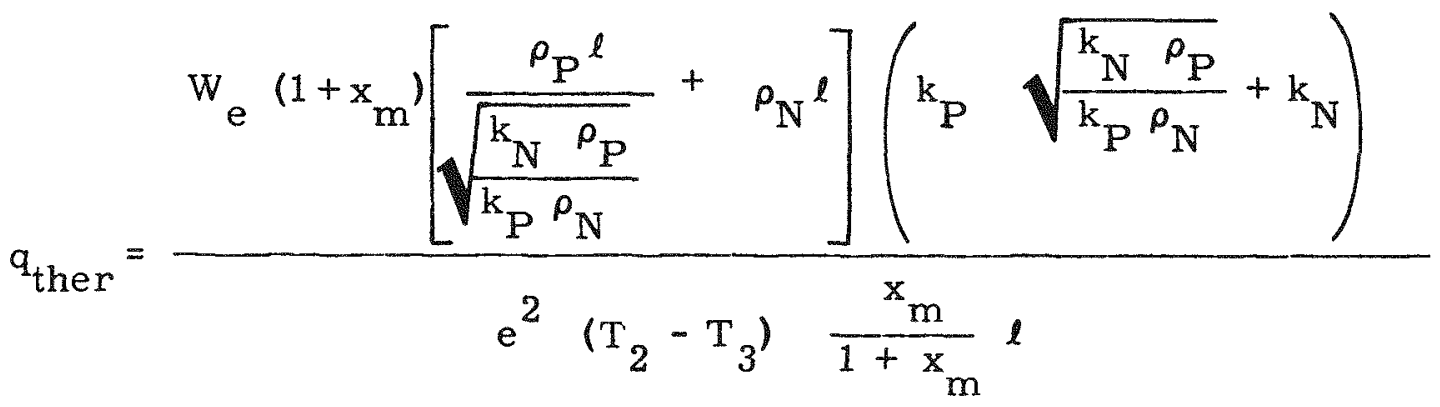

$$
A_{3}=\frac{\frac{4 W_{e}}{\eta_{e l} \eta_{t h}}}{4 \epsilon_{\text {ir }} \sigma T_{3}^{4}-\left(1-\alpha_{v}\right) 1400\left(\mu+\frac{\alpha_{E}}{\pi} \cos \xi\right)-188.5 \epsilon_{\text {ir }}}
$$


$C-6$

where

$\epsilon_{\text {ir }}=$ the infrared emissivity of the cold junction surface

$\sigma \quad=$ the Stefan-Boltzmann constant (watts $/ m^{2}-{ }^{\circ} K^{4}$ )

$\left(1-\alpha_{\mathrm{V}}\right)=$ the absorptivity of the surface for solar radiation

$\mu \quad=$ the orbit orientation factor (equals 1 for polar orbit)

$\alpha_{E} \quad=$ the absorptivity for radiation from the earth

$\cos \xi=$ the cosine of the geocentric angle of the satellite's orbit. All units are watts, meters and ${ }^{\circ} \mathrm{K}$.

$A_{2}=\pi\left(\sqrt{\frac{A_{3}}{\pi}}-2[\ell+1.27]\right)^{2}$

where

$\ell$ is in centimeters: 1.27 refers to the distance required for mounting the thermoelectrics.

$q_{\text {ins }}=\frac{\mathrm{k}_{\text {ins }}\left(\mathrm{T}_{2}-\mathrm{T}_{3}\right) 4 \sqrt{\pi \mathrm{A}_{\text {ther }}}}{\left[\ln \left(\frac{2 \mathrm{r}_{3} \sqrt{\pi}-\sqrt{\mathrm{A}_{\text {ther }}}}{2 \mathrm{r}_{3} \sqrt{\pi}+\sqrt{\mathrm{A}_{\text {ther }}}}\right)-\ln \left(\frac{2 \mathrm{r}_{2} \sqrt{\pi}-\sqrt{\mathrm{A}_{\text {ther }}}}{2 \mathrm{r}_{2} \sqrt{\pi}+\sqrt{\mathrm{A}_{\text {ther }}}}\right)\right]}$

where

$$
\begin{aligned}
& \mathrm{k}_{\text {ins }}=\text { the thermal conductivity of the insulation } \\
& \mathrm{r}_{3} \text { and } \mathrm{r}_{2}=\text { the radii of cold and hot junction spheres, respectively. } \\
& \eta_{\text {th }}=\frac{1}{1+\frac{q_{\text {ins }} \eta_{\mathrm{el}}}{\mathrm{w}_{\mathrm{e}}}} \\
& \mathrm{q}_{\mathrm{s}}=\mathrm{q}_{\mathrm{ins}}+\mathrm{q}_{\text {ther }}+\mathrm{q}_{\mathrm{res}} \\
& \mathrm{q}_{\mathrm{s}}=\frac{W_{\mathrm{e}}}{\eta_{\mathrm{el}} \eta_{\text {th }}}
\end{aligned}
$$




$$
A_{1}=\frac{A_{2} q_{s}}{1\left[\sigma A_{2}\left(T_{1}^{4}-T_{2}^{4}\right)-\left(\frac{1}{{ }_{2}}-1\right) q_{S}\right]}
$$

where

- = the surface emissivity and subscripts 1 and 2 refer to the source cladding surface and hot junction, respectively.

$w_{s}=\frac{w_{e}}{\eta_{t h} \eta_{e l}} \frac{\rho_{s}}{P}$

where

$\rho_{\mathrm{S}} \quad=$ the source density $\left(\mathrm{gm} / \mathrm{cm}^{3}\right)$
$P \quad=$ the power density (watts $/ \mathrm{cm}^{3}$ )

$w_{c}=\left[\frac{\pi D_{1}^{3}}{6}-\left(\frac{w_{e}}{\eta_{e l} \eta_{t h} P}+\frac{q_{s}(12)}{100}\right)\right] \rho_{c l a d}$

where

$D_{1} \quad=$ the diameter of the source sphere

$q_{S}(12)=$ the thermal power calculated in Eq (12)

$\frac{q_{S}(12)}{100}=$ a void space to allow for helium accumulation.

$w_{\text {sup }}=\left(D_{2}-D_{1}\right) A_{\text {sup }} \rho_{\text {sup }}$

where

$A_{\text {sup }}=$ the cross-sectional area of the source supports

$\rho_{\text {sup }}=$ the density of the supports.

$w_{\text {i.s. }}=\pi D_{2}^{2} t_{i . s .} \rho_{i . s .}$ 
where

$$
\begin{aligned}
& t_{i . s .}=\text { the thickness of the inner shell (hot junction) } \\
& \rho_{i_{. s} s}=\text { the density of the shell. } \\
& w_{\text {ther }}=A_{\text {ther }} \ell \rho_{\text {ther }}
\end{aligned}
$$

where

$$
\begin{aligned}
& p_{\text {ther }}=\text { the thermoelectric density. } \\
& \text { wins }_{\text {in }}=\left(\pi D_{2}^{2}-A_{\text {ther }}\right) \ell \rho_{\text {ins }}
\end{aligned}
$$

where

$$
\begin{aligned}
& \rho_{\text {ins }}=\text { the insulation density. } \\
& w_{\text {o.s. }}=\left(\pi D_{3}\right)^{2} t_{\text {o.s. }} \rho_{\text {o.s. }}
\end{aligned}
$$

where

$$
\begin{aligned}
& t_{\text {O.S. }}=\text { the outer shell thickness } \\
& \rho_{\mathrm{O} . \mathrm{S} .}=\text { the outer shell density. } \\
& \text { Total weight }=w_{s}+w_{c}+w_{\text {sup }}+w_{\text {i.s. }}+1.25 w_{\text {ther }}+w_{\text {ins }}+w_{0 . s .}
\end{aligned}
$$

\section{GENERATOR DESIGNS}

In addition to the computer effort, some attention has been devoted to an

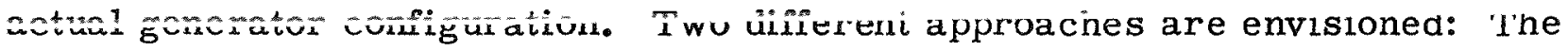
first is to attempt use of a standard heat dump mechanism such as a thermal shutter. The second, which will eliminate heat control problems, is to overdesign the generator with a dump of the excess electrical power. Although this may seem to be somewhat less efficient than the the rmal dump design, reliability may be increased at the same overall weight. In addition, the more uniform temperature distribution on the hot junction may cause enough of an improvement in efficiency to partially offset the drop caused by the lower hot junction temperature.

The following is the FORTRAN listing of the "Space Power Source Program." 


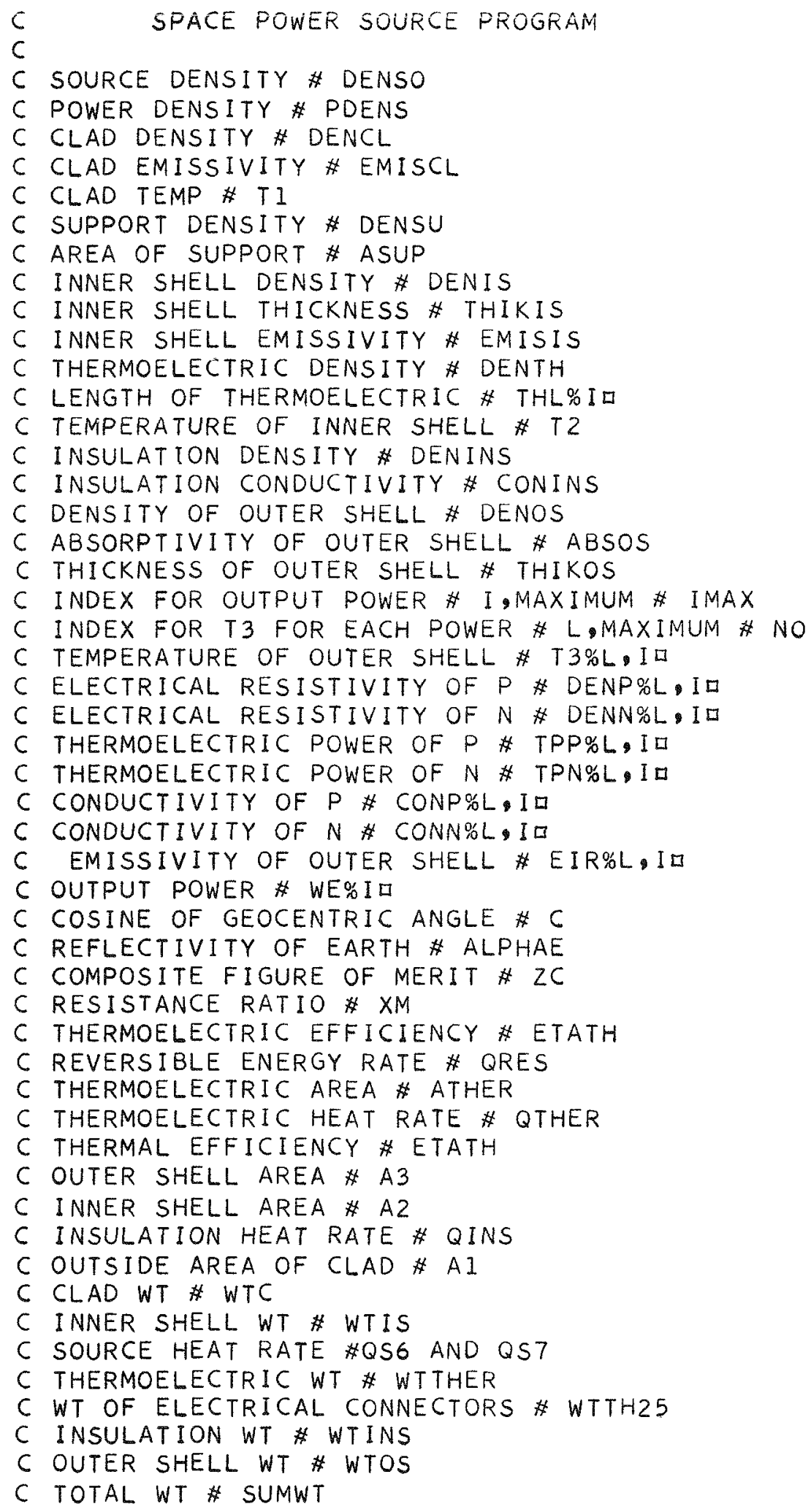


C ECLIPSE FACTOR \# U

C CONVERGENCE CRITERION FOR THERMAL EFFICIENCY \# EPSI

C

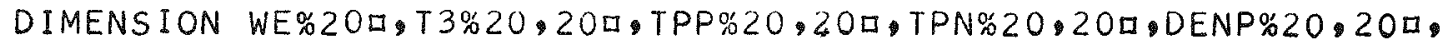

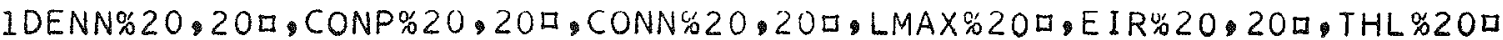

READ 200,PDENS,DENSO

READ 201,DENCL,EMISCL,TI

READ $200, D E N S U, A S U P$

READ 201,DENIS, THIKIS, EMISIS

READ 200,DENTH,T2

READ 200,DENINS, CONINS

READ 201,DENOS, ABSOS,THIKOS

READ 207, ETATH,EPS,NOUT,NEXT

READ 201: ALPHAE,U,C

READ 203, IMAX

DO 205 I I. IMAX

READ 206, THL\%I

READ 208,WE\% ID

READ 204,\%T3\%L, 1口,L\#I,NOD

READ 204,\%TPP\%L,Iロ,L\#I,NOU

READ 204\%\%TPN\%L,ID,L\#1,NOロ

READ 204,\%DENP\%L,ID,L\#1,NOD

READ 204\% \%ENN\%L ID,L\#1,NOD

READ $204 \%$ CONP\%L, ID,LWI,NOD

READ 204\% CONN\%L, ID, L\#1,NOD

READ 204\% \% IR\%L,Iロ,L\#I,NOD

LMAX\%IロWNO

205 CONTINUE

200 FORMAT\%1P2E12.5口

201 FORMAT\%1P3E12.5口

202 FORMAT\%1P4E12.5口

203 FORMAT\% I12

204 FORMAT\%1P6E12.5口

206 FORMAT\%1PE12.5,I12

207 FORMAT\%IP2EI2.5.2I12口

208 FORMAT\%1PE 12.5口

$A B O \# A B S O S$

WRITE OUTPUT TAPE 10,300

300 FORMAT\% 43 HI DESIGN PARAMETERS FOR SPACE POWER SOURCES///12H INPUT IDATA 口 WRITE OUTPUT TAPE 10,301, PDENS, DENSO

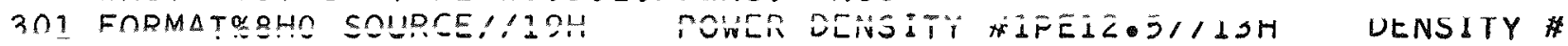
11PE 12.5口

WRITE OUTPUT TAPE $10,302, D E N C L, E M I S C L, T 1$

302 FORMAT\%6HO CLAD//I3H DENSITY \#1PE $12.5 / / 16 \mathrm{H}$ EMISSIVITY \#IPE12 1.5//8H TI \#PE12.5口

WRITE OUTPUT TAPE 10,303, DENSU,ASUP

303 FORMAT\%9HO SUPPORT//13H DENSITY \#1PE12.5//10H WRITE OUTPUT TAPE 10,304, DENIS,THIKIS,EMISIS

304 FORMAT\%13HO INNER SHELL//13H DENSITY \#1PE12.5//15H

AREA \#1PE12.5口

1 \#1PE12.5//16H EMISSIVITY \#1PE12.5口

WRITE OUTPUT TAPE 10,305, DENTH,T2

305 FORMAT\%16HO THERMOELECTRIC//13H DENSITY \#1PE12.5//8H

THICKNESS

T2 \#IPEI2. 
150

WRITE OUTPUT TAPE 10,306 DENINS, CONINS

306 FORMAT\%12HO INSULATION//13H DENSITY

ITY \#IPE12.5口

WRITE OUTPUT TAPE 10,307, DENOS,ABSOS,THIKOS

307 FORMAT\%13HO OUTER SHELL//13H DENSITY \#IPE12.5//18H ABSORPTIV DO 309 I\# I, IMAX

NO\#LMAX\% I

IITY \#1PE12.5//15H THICKNESS \#1PE12.5口

WRITE OUTPUT TAPE 10,308,WE\%Iロ,THL

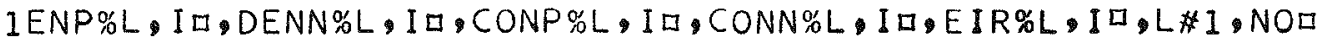

308 FORMAT\% 16 HO OUTPUT POWER \#IPE $12.5 / / 17 H 0$ THERMO LENGTH \#IPEI2.5//72 IH T3 TP-P TP-N DEN-P DEN-N CON-P CON-N

2EMIS $/ 1 \% 1 P 8 E 9.2$ ㅁ

309 CONTINUE

WRITE OUTPUT TAPE 10,3091 , ALPHAE,U,C

3091 FORMAT\%16HO REFLECTIVITY \#1PE12.5//18H ECLIPSE FACTOR \#1PEI2.5//I $10 \mathrm{H}$ COSINE \#IPE12.5口

$I \not 1$

8 WRITE OUTPUT TAPE 10,9, WE\%ID

9 FORMAT\%I6HI OUTPUT POWER \#IPE12.5口

IF\%SENSE SWITCH 3ם41,40

41 PRINT 9,WE\% I

40 L

STORE\#ETATH

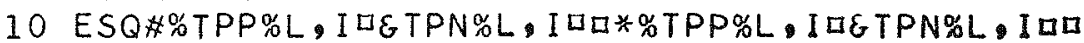

ZC\#ESQ /\% \% SQRTF\%CONP\%L, I

$12 . \square$

EPS1\#1.E15

DELTHT2-T3\%L, ID

$X M \# S Q R T F \% 1 . \varepsilon \% T 2 E T 3 \square / 2 \cdot \square * Z C \square$

$X M I \# I \cdot E X M$

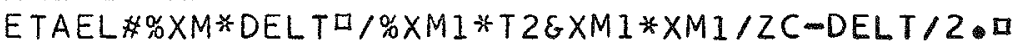

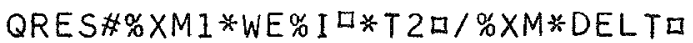

SR \#SQRTF\% \%CONN\%L, I

FACTOR \%\%DENP\%L, I

$1 \square / \% W E \% I \square * X M 1 * X M 1 \square \square$

ATHER FACTOR*\% I \&SR口

QTHER \#FACTOR*\%CONP\%L, I 口*SRECONN\%L,I吅*DELTロ/THL\%ID

SI GMA $\$$.67E-8

IF\%SENSE SWITCH 3ם15,20

15 PRINT 151

151 FORMAT\%13HO THERMAL EFF//

NA\#1

20 ETATHI ETATH

NA \#NAEI

THINS\#THLEI.27

EPS2 \#EPS1

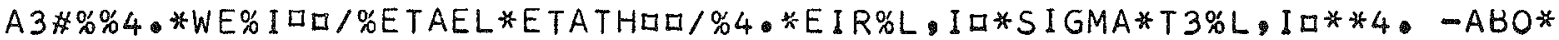

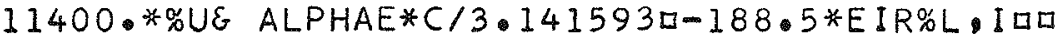

$A 3 * A 3 * 1, E 4$

A2*3.141593*\% SQRTF\%A3/3.141593ロ-2•*THINSם**2.口 


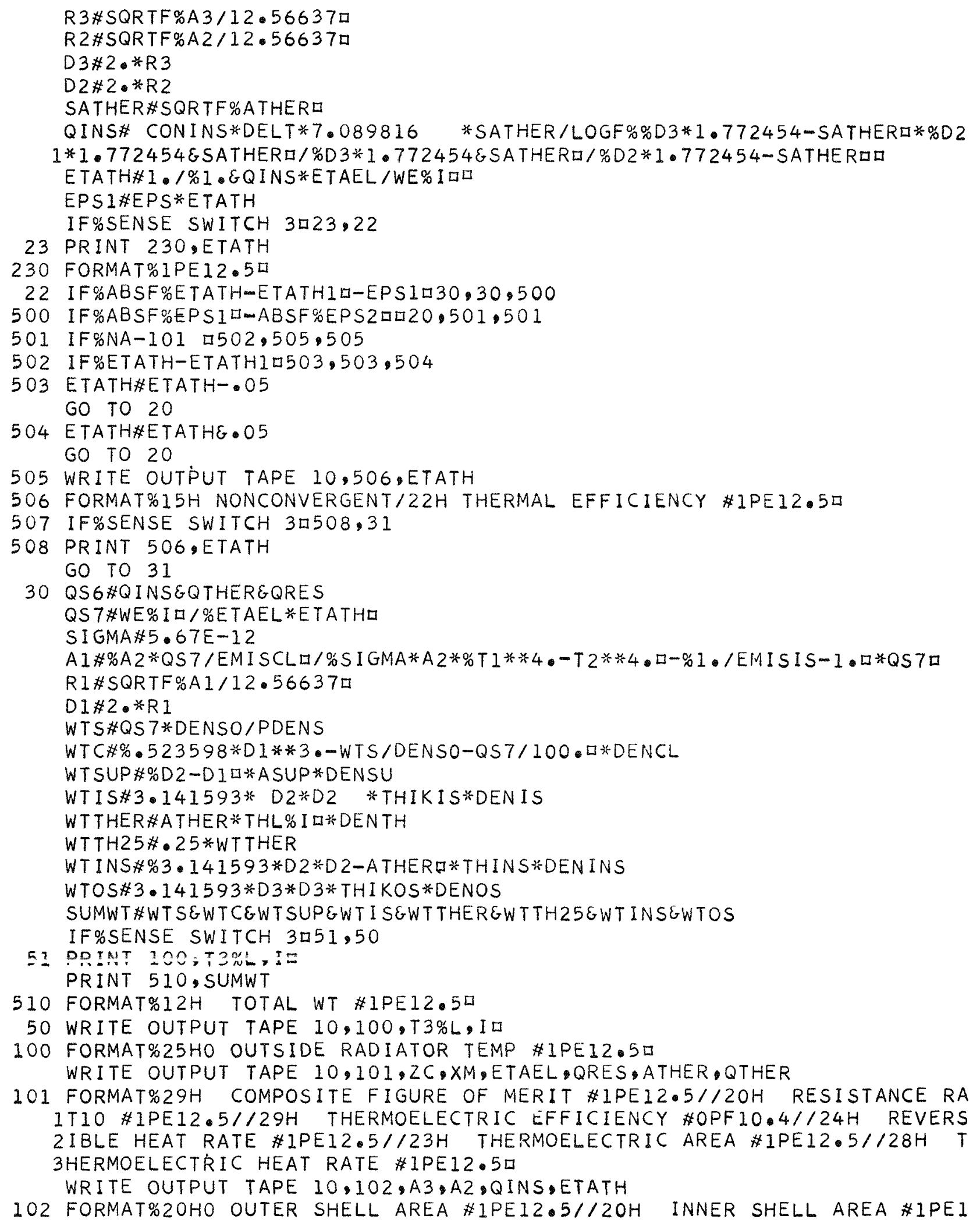


12.5/124H INSULATION HEAT RATE \#IPE12.51/22H THERMAL EFFICIENCY \# 2OPF $20.4 \square$

WRITE OUTPUT TAPE $10,103, Q S 6, Q S 7, A 1, W T S, W T C, W T S U P, W T$ IS,WTTHER,WTTH 125 , WTINS, WTOS, SUMWT

103 FORMAT\%25HO SOURCE HEAT RATE,EQ.6 \#1PE12.5//25H SOURCE HEAT RATE, IEQ.7 \#IPE12.5//24H OUTSIDE AREA OF CLAD \#IPE12.5//13H SOURCE WT 2\#1PE12.5//11H CLAD WT \#1PE12.5//14H SUPPORT WT $\$ 1 P E 12.5 / / 18 \mathrm{H}$ IN 3NER SHELL WT \#1PE12.5//21H THERMOELECTRIC WT \#1PE12.5//31H WT OF 4 ELECTRICAL CONNECTORS \#1PE12.5//17H INSULATION WT \#1PE12.5//18H

5 OUTER SHELL WT \#1PE12.5//12H TOTAL WT \#1PE12.5口 ETATH\#STORE

1030 IF\%L-LMAX\%Iㅁ60,31,98

60 L\#LE 1

GO TO 10

98 PRINT $1098, \mathrm{~L}$

1098 FORMAT\%4HI L\#I12,36H,TOO BIG-STATEMENT 1030. CHECK INPUTD GO TO 1000

31 IF\%I-IMAXロ $32,33,99$

$32 I \# I E I$

GO TO 8

99 PRINT 1099,1

1099 FORMAT\%4HI I $112,34 \mathrm{H}$, TOO BIG-STATEMENT 31. CHECK INPUTE GO TO 1000

33 WRITE OUTPUT TAPE 10,104

104 FORMAT\%9H1 THE ENDI

1000 CONTINUE

$Y \# F M P B F \% X \square$

STOP

END\%0,1,0,0,1口 


\section{APPENDIX D}

\section{SUBTASK 5. 7--SHIELDING CALCULATIONS METHODS}

1. Albert-Welton Kernel Method

$$
\begin{aligned}
& \text { neutron flux }=\text { constant } x \mathrm{e}^{-\Sigma \mathrm{x}} \text { point source; } \\
& \text { neutron flux }=\frac{\text { constant }}{4 \pi \mathrm{r}^{2}} \mathrm{e}^{-\Sigma \mathrm{x}}
\end{aligned}
$$

where

$$
\begin{aligned}
& x=\text { shield thickness } \\
& \Sigma=\text { a constant }=\text { removal cross section } \\
& r=\text { distance from point source } \mathrm{x}
\end{aligned}
$$

The theoretical hydrogen total cross section is given by

$\sigma_{H}=\frac{3 \pi}{4}\left[K^{2}+\left(\frac{-1}{a_{t}}+\frac{1}{2} n_{t} K^{2}\right)^{2}\right]^{-1}+\frac{\pi}{4}\left[K^{2}+\left(\frac{-1}{a_{s}}+\frac{1}{2} n_{s} K^{2}\right)^{2}\right]^{-1} \mathrm{~cm}^{2}$

where $\mathrm{K}=$ wave number of neutron in center-of-mass system $=\frac{1}{\lambda}$

$$
\begin{array}{ll}
a_{t}=0.528 \times 10^{-12} \mathrm{~cm} & r_{s}=1 \times 10^{-13} \mathrm{~cm} \quad \lambda=2.86 \times 10^{-9} \sqrt{\mathrm{E} \mathrm{ev}} \\
a_{s}=2.375 \times 10^{-12} \mathrm{~cm} & r_{t}=1.56 \times 10^{-13} \mathrm{~cm}
\end{array}
$$

A good approximation between 2 and $12 \mathrm{Mev}$ is

$$
\sigma_{H}=\frac{5.13}{E^{0.725}} \text { barns } E \text { in Mev }
$$

If the neutron fission spectrum as empirically determed by Los Alamos is multiplied by the Albert-Welton neutron attenuation formula the hardened fission spectrum at point $\mathrm{x}$ is determined. Thus,

$$
N(x)=0.484 e^{-E} \sinh \sqrt{2 E} e^{-\Sigma x}
$$


where

$$
\begin{aligned}
\Sigma=\frac{\sigma_{\mathrm{H}} \mathrm{N}_{\mathrm{a}} \rho_{\mathrm{H}}}{\mathrm{A}} & =\underset{\text { tion }}{\text { macroscopic hydrogen removal cross sec }-} \\
\mathrm{N}_{\mathrm{a}} & =\underset{\text { atom }}{\text { Avogardros number } 26.02 \times 10^{23} \text { atoms } / \mathrm{gm}-} \\
\mathrm{A} & =\text { atomic weight } \\
\rho_{\mathrm{H}} & =\text { density } \mathrm{gm} / \mathrm{cc}
\end{aligned}
$$

$\sigma_{0}=$ oxygen removal cross section, empirically determined at 0.91 barn. This hardened spectrum for 10 inches of water is shown in Fig. D-1. The dose rate in $\mathrm{mrem} / \mathrm{hr}$ versus neutron energy on a one neutron basis is given in Fig. D-2. Thus, the total neutron dose over neutron energies from 0.7 to $10 \mathrm{Mev}$ can be determined by graphical integration of Fig. D-3.

\section{Moments Method}

The Boltzmann equation was solved by the method of moments for penetration of neutrons from a point isotropic fission source in water by $R$. Aronson. This method consists of replacing the Boltzmann equation for the flux from a plane isotropic fission source by a set of simultaneous integral equations. These equations are in terms of the energy variable lethargy $\left(=\log \frac{10 \mathrm{MeV}}{\mathrm{E}}\right)$ for the spatial moments of the Legendre coefficients of the flux. $E_{0}=$ arbitrary fixed energy that was chosen as $0.33 \mathrm{Mev}$ for convenience.

Hydrogen collisions with neutrons were taken as elastic scattering that is isotropic in a center-of-mass system. All oxygen collisions other than elastic scattering were considered absorptions. Scattered

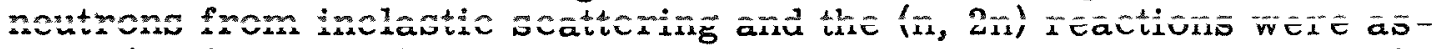
sumed to have too low an energy to contribute to the total neutron dose. The dose versus shield thickness for both water and hydrogen (density $=0.111 \mathrm{gm} / \mathrm{cc}$ ) is shown in Fig. D-4. 


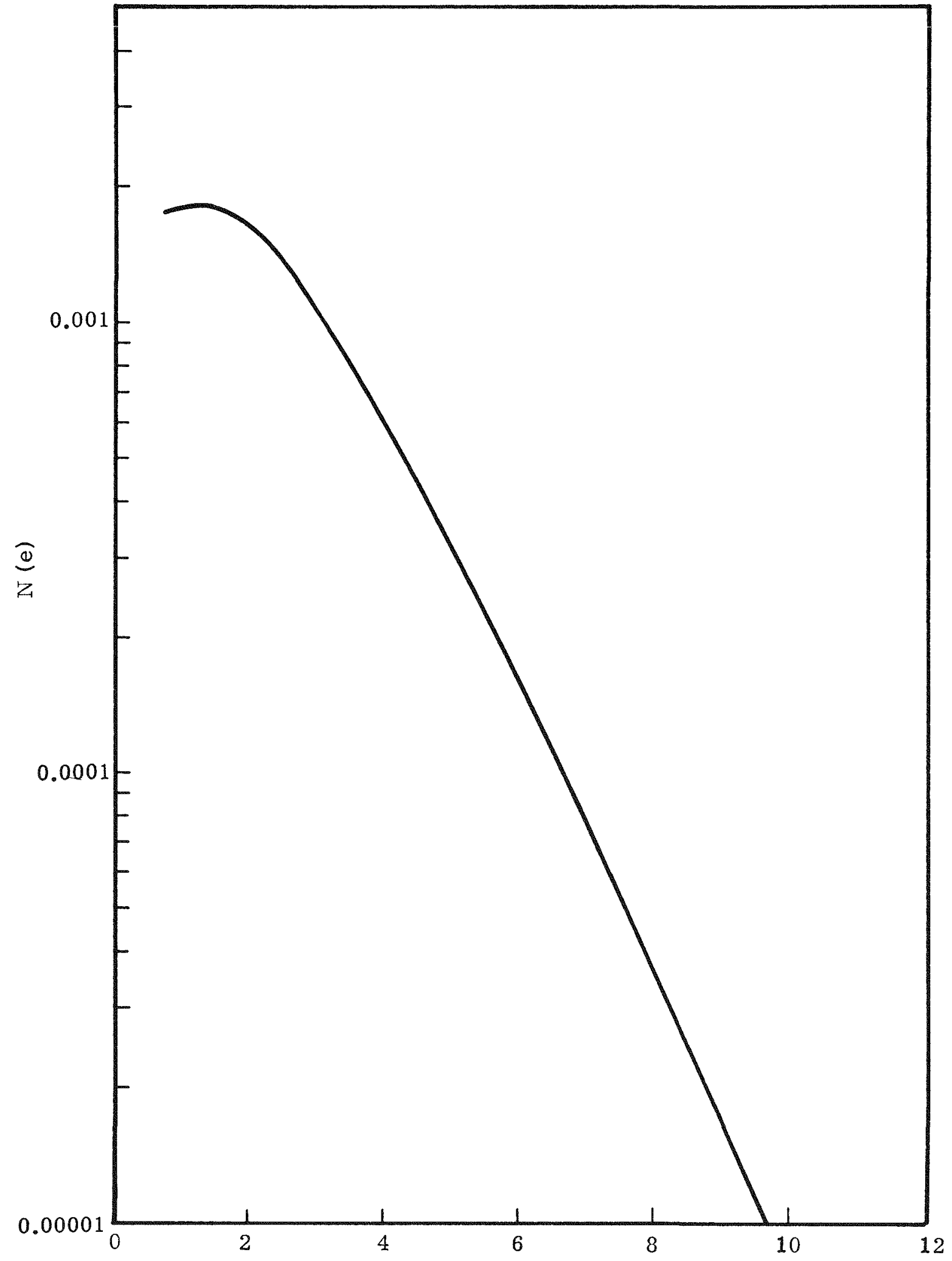

Fig. D-1. Hardened Fission Spectrum --10 in. $\mathrm{H}_{2} \mathrm{O}$ 
D-4

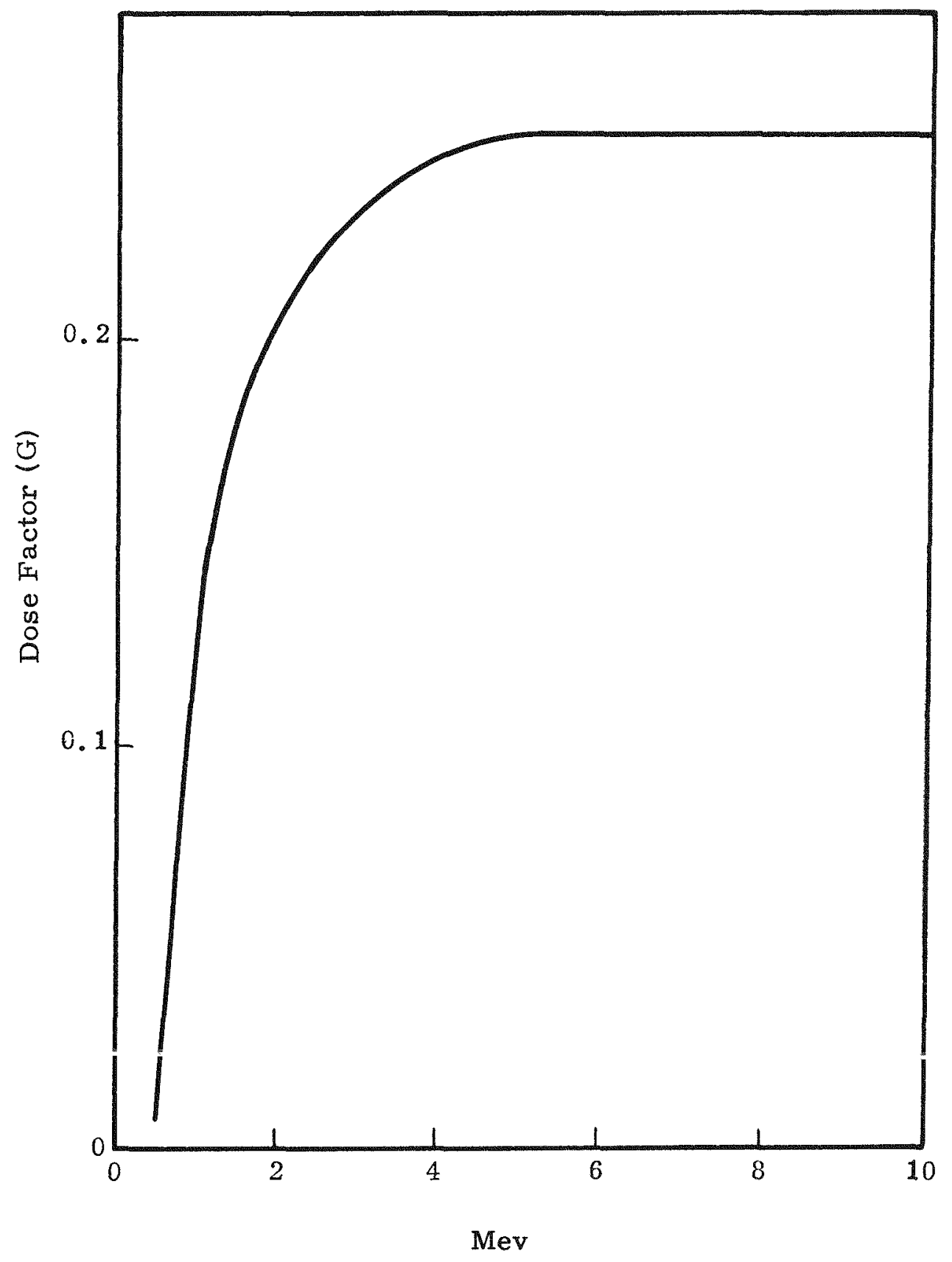

Fig. D-2. Dose Factor Versus Energy Level--One Neutron 


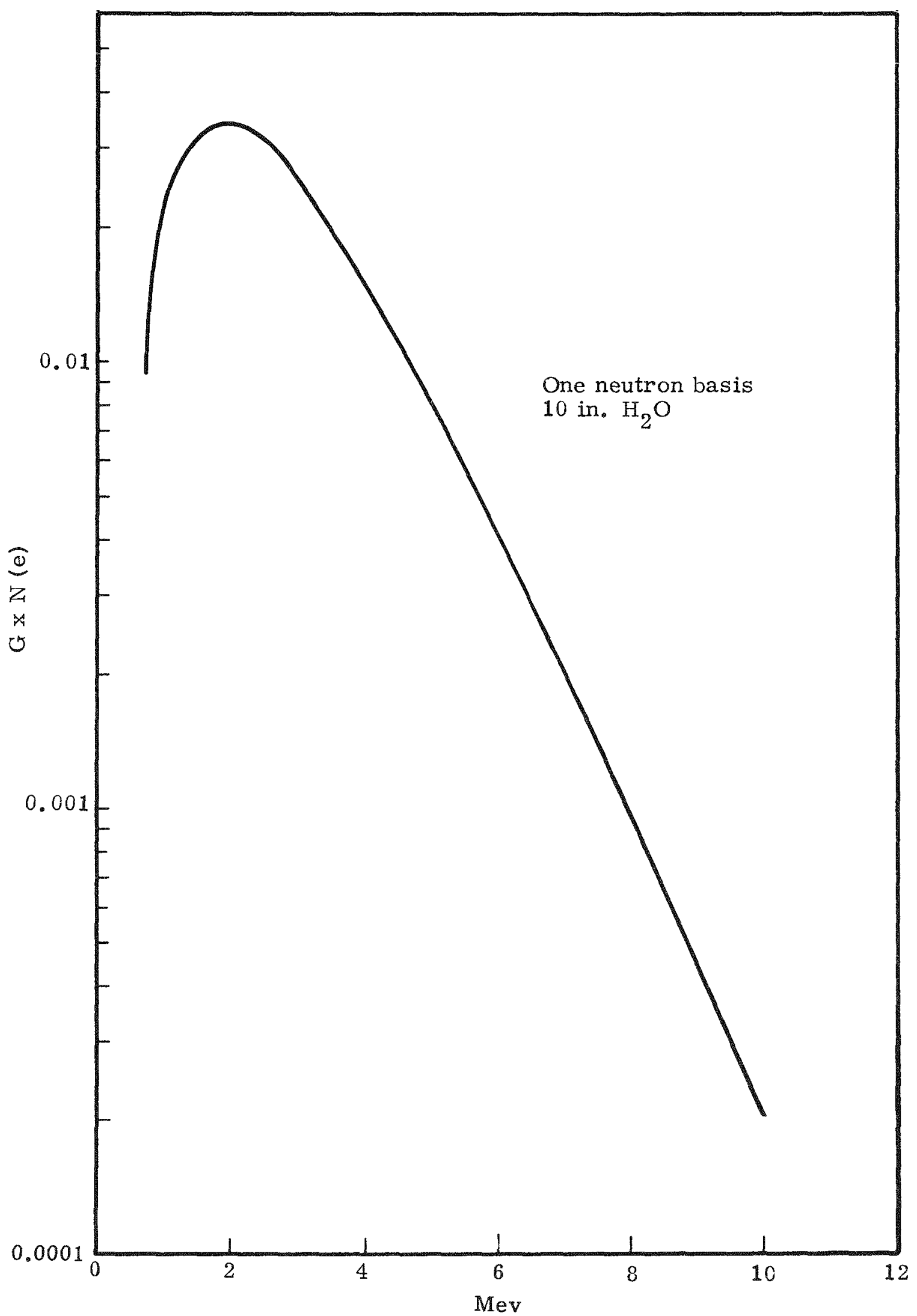

Fig. D-3. Product of Hardened Fission Spectrum and Biological Dose Factor 
D-6

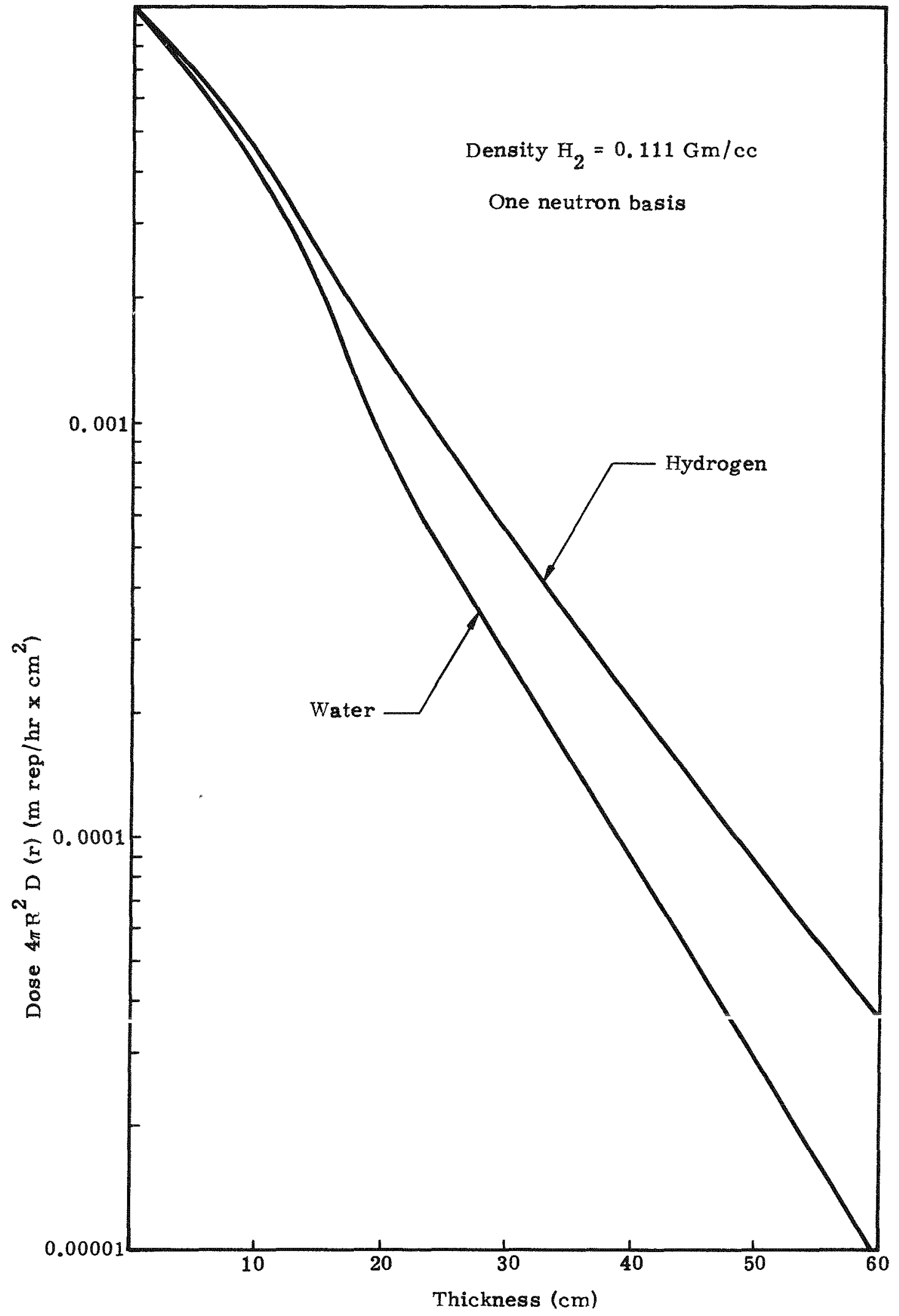

Fig. D-4. Dose Versus Shield Thickness 


\section{APPENDIX E}

\section{HEAT FLOW CALCULATIONS}

$$
\begin{aligned}
& \text { Singer's* equation is: } \\
& \qquad T_{r}=\left[\frac{\left(1-\alpha_{v}\right)(1400)\left(\mu+\frac{\alpha_{E}}{\pi} \cos \rho\right)+{ }_{i r}(188.5)+x}{4{ }_{i r}{ }^{\sigma}}\right]^{1 / 4}
\end{aligned}
$$

where

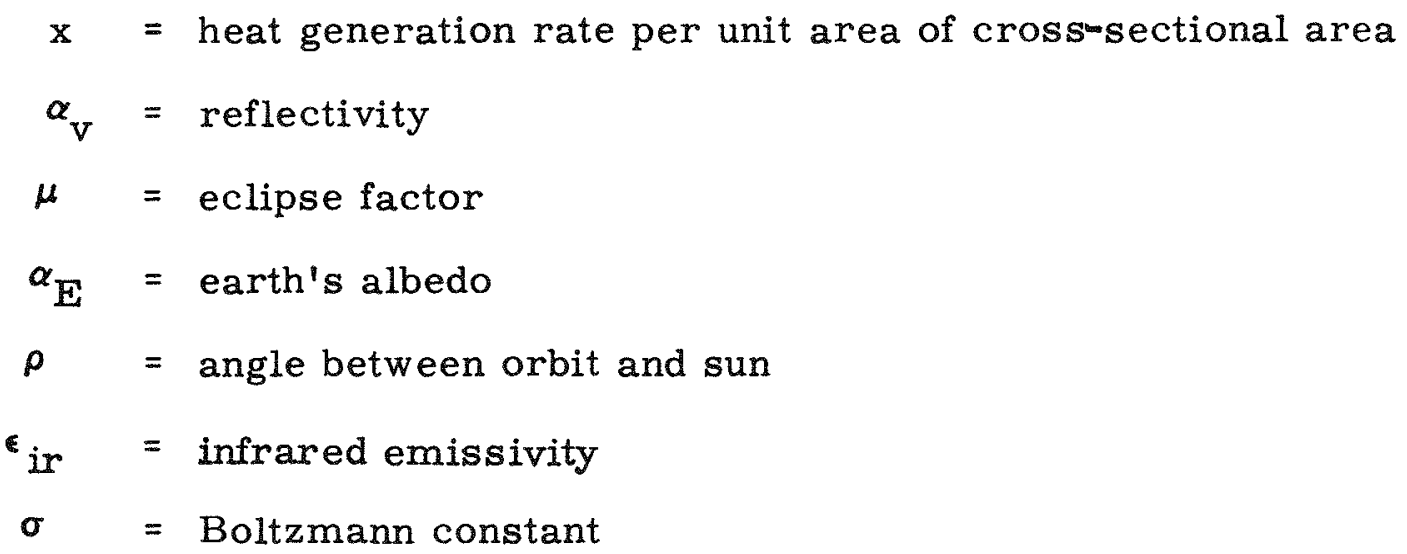

The values assumed in this equation were

$$
\begin{aligned}
& 1-\alpha_{\mathrm{v}}=0.1 \\
& \mu+\frac{\alpha_{\mathrm{E}}}{\pi} \cos \rho=0.7 \\
& { }_{\mathrm{ir} r}=0.94
\end{aligned}
$$

1. Heat Loss--Radiative Unit

The radial heat flow through a sphere is given by:

$$
q=4 \pi k\left(\frac{t_{1}-t_{2}}{r_{2}-r_{1}}\right) r_{1} r_{2}
$$

\section{Heat Loss--Scaled-up Unit}

The radial heat flow through a combined sphere and cylinder is 


$$
q=4 \pi k\left(\frac{t_{1}-t_{2}}{r_{2}-r_{1}}\right) r_{1} r_{2}+k \frac{\left(A_{2}-A_{1}\right)}{\ln \left(A_{2} T A_{1}\right)}\left(\frac{t_{1}-t_{2}}{r_{2}-r_{1}}\right)
$$

Simplifying, this becomes

$$
\begin{aligned}
& \mathrm{q}=\mathbf{k}\left(\frac{\mathrm{t}_{1}-\mathrm{t}_{2}}{\mathrm{r}_{2}-\mathrm{r}_{1}}\right)\left[4 \pi \mathrm{r}_{1} \mathrm{r}_{2}+\frac{\mathrm{A}_{2}-\mathrm{A}_{1}}{\ell \mathrm{n}\left(\mathrm{A}_{2} / \mathrm{A}_{1}\right)}\right] \\
& \mathrm{q}=\mathrm{Btu} / \mathrm{hr} \\
& \mathbf{k}=\text { thermal conductivity } \\
& \mathrm{t}_{1}=\text { temperature at } \mathrm{r}_{1} \\
& \mathrm{t}_{2}=\text { temperature at the surface }\left(\mathrm{r}_{2}\right) \\
& r_{1}=\text { distance from the center to the inner shell } \\
& r_{2}=\text { distance from center to the surface } \\
& A_{1}=\text { cylindrical surface area of inner shell } \\
& A_{2}=\text { cylindrical surface area of outer shell }
\end{aligned}
$$

The thermoelectric calculations for this design were achieved using the equations derived by $R$. Ningard, therefore, these will not be discussed. Heat loss--Cylindrical Unit.

\section{Heat Loss--Cylindrical Unit}

Fourier's law for conduction through a homogeneous solid is given as

$$
q=-k A(r) \frac{d T(r)}{d r}
$$

where

$$
\begin{aligned}
& \mathrm{q}=\text { heat flow } \\
& \mathrm{k}=\text { thermal conductivity } \\
& \mathbf{r}=\text { radius or thickness } \\
& \mathrm{A}=\text { area as a function of position }
\end{aligned}
$$


$\mathrm{T}=$ temperature as a function of position

Integration between the inner and outer surfaces gives

$$
\int_{r_{1}}^{r_{2}} \frac{d r}{A(r)}=\frac{k}{q}\left(T_{1}-T_{2}\right)
$$

where

$$
\begin{aligned}
& r_{1}=\text { distance from center to inner shell } \\
& r_{2}=\text { distance from center to outer shell } \\
& T_{1}=\text { inner shell temperature } \\
& T_{2}=\text { outer shell temperature }
\end{aligned}
$$

This equation can be used to compute the heat loss when the area term has been expressed so as to include heat flow through the sides and ends.

The heat dump calculations were performed using Fourier's third law and considering Fig. 11.

$q=-k A \frac{\Delta t}{\Delta x}$

$\mathbf{k}=$ thermal conductivity of combined Min-K and helium ga at 1 atmosphere of pressure

$A=$ cross-sectional area normal to the heat flow

$\Delta t=$ temperature difference between inside and outside shell

$\Delta \mathrm{x}=$ distance through the wall of the dump

The combined Min-K and helium gas thermal conductivity was approximated by

$$
\mathrm{k}=\mathrm{k}_{1}+\left(\mathrm{k}_{2}-\mathrm{k}_{1}\right) \frac{\mathrm{k}_{4}}{\mathrm{k}_{3}}
$$


where

$k_{1}=$ Min $-K$ thermal conductivity at vacuum

$k_{2}=$ Min-K thermal conductivity at 1 atmosphere in air

$\mathrm{k}_{3}=$ air thermal conductivity at 1 atmosphere

$\mathbf{k}_{4}=$ helium thermal conductivity at 1 atmosphere

When the design power has been determined, the initial power necessary to allow for isotope decay can be calculated by:

$$
N_{o}=N_{d} e^{\lambda t}
$$

where

$$
\begin{aligned}
& \mathrm{N}_{\mathrm{o}}=\text { initial power } \\
& \mathrm{N}_{\mathrm{d}}=\text { design power } \\
& \lambda=0.693 / \text { half life } \\
& \mathrm{t}=\text { time }
\end{aligned}
$$

The initial isotope loading can then be determined using the known power density.

The fuel element temperature was calculated using the equation for long concentric cylinders given as

$$
Q=\frac{\sigma A_{1}\left(T_{1}^{4}-T_{2}^{4}\right)}{\frac{1}{1}+\frac{A_{1}}{A_{2}}\left(\frac{1}{6}-1\right)}
$$

where

$$
\begin{aligned}
& Q=\text { heat flow rate } \\
& \sigma=\text { Stefan-Boltzmann constant }
\end{aligned}
$$


$A_{1}=$ area of inner cylinder

$A_{2}=$ area of outer cylinder

$\epsilon_{1}=$ emissivity of inner cylinder

${ }_{2}=$ emissivity of outer cylinder

$T_{1}=$ temperature of inner cylinder

$\mathrm{T}_{2}=$ temperature of outer cylinder 


\section{APPENDIX F}

\section{CALCULATION OF SURFACE TEMPERATURE}

The density of curium is $13.5 \mathrm{gm} / \mathrm{cc}$ and that of nickel is $8.9 \mathrm{gm} / \mathrm{cc}$. The power density of pure curium metal is 120 thermal watts/gm or 1620 thermal watts $/ \mathrm{cc}$. The surface temperature of a fuel pellet of 1000 thermal watts output and consisting of 20 parts $\mathrm{Ni}$ to 1 part $\mathrm{Cm}$ by weight is determined as follows:

$$
\begin{aligned}
& \frac{1000 \text { watts }}{120 \text { watts } / \mathrm{gm}}=8.33 \mathrm{gm} \mathrm{Cm} \\
& \mathrm{V}_{\mathrm{cm}}=\frac{8.33}{13.5}=0.616 \mathrm{cc} \mathrm{Cm} \\
& \mathrm{W}_{\mathrm{ni}}=8.33 \times 20=166.6 \mathrm{gm} \mathrm{Ni} \\
& \mathrm{v}_{\mathrm{ni}}=\frac{166.6}{8.9}=18.7 \mathrm{cc} \mathrm{Ni}
\end{aligned}
$$

$0.616+18.7=19.3 \mathrm{cc} \mathrm{Ni}-\mathrm{Cm}$ alloy $(100 \%$ theoretical wt density)

for an $\ell / d$ ratio of 1 to 1

$$
V=\pi r^{2} \ell=2 \pi r^{3}
$$

where

$$
\begin{aligned}
\ell & =\mathrm{d}=2 \mathrm{r} \\
\mathrm{A} & =2 \pi \mathrm{r}^{2}+2 \pi \mathrm{r} \ell=6 \pi \mathrm{r}^{2}
\end{aligned}
$$

where
$A=$ total surface area
$\mathrm{V}=$ volume
$\ell=$ height of pellet
$d$ = diameter
$\mathbf{r}=$ radius 
F-2

$$
\begin{aligned}
& v=19.3=2 \pi \mathrm{r}^{3} \\
& \mathrm{r}^{3}=\frac{19.3}{2 \pi}=3.08 \\
& \mathrm{r}=1.455 \mathrm{~cm} \\
& \mathrm{~A}=6 \pi \mathrm{r}^{2}=6 \pi(1.455)^{2}=40 \mathrm{~cm}^{2}
\end{aligned}
$$

For a radiant boundary with infinite surroundings,

$$
\mathrm{Q}=\sigma \in \mathbb{A}\left(\mathrm{T}_{1}^{4}-\mathrm{T}_{2}^{4}\right)
$$

where

$Q=$ heat transferred (cal/sec)

$\sigma=$ Stefan-Boltzmann constant $\left(\mathrm{cal} / \mathrm{sec}-\mathrm{cm}^{2}-{ }^{\circ} \mathrm{K}^{4}\right)$

- = surface emissivity (dimensionless)

$\mathrm{T}_{1}=$ source temperature $\left({ }^{\circ} \mathrm{K}\right)$

$\mathrm{T}_{2}=$ sink temperature $\left({ }^{\circ} \mathrm{K}\right)$

$$
\mathrm{T}_{1}^{4}=\frac{\mathrm{Q}}{\sigma \Leftrightarrow \mathrm{A}}+\mathrm{T}_{2}^{4}
$$

$=1000$ watts $(3.415 \mathrm{Btu} / \mathrm{hr} / \mathrm{watt})(252 \mathrm{cal} / \mathrm{Btu})$

$\left(0.1357 \times 10^{-11} \mathrm{cal} / \mathrm{sec}-\mathrm{cm}^{2}-^{\circ} \mathrm{K}^{4}\right)(0.85)\left(40 \mathrm{~cm}^{2}\right)\left(3600 \frac{\mathrm{sec}}{\mathrm{hr}}\right)$

$$
+(308)^{4}
$$

$=5.2 \times 10^{12}+90 \times 10^{8}=5.2 \times 10^{12}$

$$
\mathrm{T}_{1}=1237 \mathrm{C}
$$

